\title{
Three-Dimensional Multispecies Distribution Functions In A Plasma Boundary With An Oblique Magnetic Field
}

Derek S Thompson

Follow this and additional works at: https://researchrepository.wvu.edu/etd

\section{Recommended Citation}

Thompson, Derek S, "Three-Dimensional Multispecies Distribution Functions In A Plasma Boundary With An Oblique Magnetic Field" (2018). Graduate Theses, Dissertations, and Problem Reports. 7266.

https://researchrepository.wvu.edu/etd/7266

This Dissertation is protected by copyright and/or related rights. It has been brought to you by the The Research Repository @ WVU with permission from the rights-holder(s). You are free to use this Dissertation in any way that is permitted by the copyright and related rights legislation that applies to your use. For other uses you must obtain permission from the rights-holder(s) directly, unless additional rights are indicated by a Creative Commons license in the record and/ or on the work itself. This Dissertation has been accepted for inclusion in WVU Graduate Theses, Dissertations, and Problem Reports collection by an authorized administrator of The Research Repository @ WVU.

For more information, please contact researchrepository@mail.wvu.edu. 
THREE-DIMENSIONAL MULTISPECIES DISTRIBUTION FUNCTIONS IN A PLASMA BOUNDARY WITH AN OBLIQUE MAGNETIC FIELD

\author{
Derek S. Thompson
}

Dissertation submitted to the Eberly College of Arts and Sciences at West Virginia University in partial fulfillment of the requirements for the degree of

Doctor of Philosophy

in

Physics

Earl E. Scime, Ph.D., Chair

Amy M. Keesee, Ph.D.

Paul A. Cassak, Ph.D.

Timothy N. Good, Ph.D.

Department of Physics and Astronomy

Morgantown, West Virginia

2018

Keywords: boundary layers, presheaths, laser induced fluorescence, helicon, radio-frequency, velocity distribution functions 


\section{Abstract \\ THREE-DIMENSIONAL MULTISPECIES DISTRIBUTION FUNCTIONS IN A PLASMA BOUNDARY WITH AN OBLIQUE MAGNETIC FIELD}

Derek S. Thompson

The physics of a weakly magnetized boundary region are investigated by immersing a $76.2 \mathrm{~mm}$ diameter stainless steel disk in a helicon plasma. The velocity distributions of ions and neutral particles are mapped in the boundary region, created in $3.6 \mathrm{mTorr}$ argon gas, $650 \mathrm{~W}$ RF power, and magnetic field $B=0.06 \mathrm{~T}$, where the magnetic field lines obliquely intersect the wall $\left(\alpha=16^{\circ}\right)$. These distributions are mapped using laser induced fluorescence in three spatial (3D) and three velocity $(3 \mathrm{~V})$ dimensions, and reveal that models neglecting any one of the velocity components omit important components of the flow field. Electrostatic probe measurements are presented and establish a typical helicon plasma discharge with electron temperature $T_{e} \approx 4.2 \mathrm{eV}$ and density $n \approx 5.5 \times 10^{17} \mathrm{~m}^{-3}$. In addition to the observed ion temperature, $T_{i} \approx 0.37 \mathrm{eV}$, these measurements indicate a plasma in which the electrons are highly magnetized (the ratio of the electron cyclotron frequency to the electron collision frequency is $\omega_{c e} / \nu_{e}=2.9 \times 10^{3}$ ) and the ions are weakly collisional (the ratio of the ion cyclotron frequency to the ion collision frequency is $\left.\omega_{c i} / \nu_{i}=0.5 \pm 0.3\right)$.

Ion drift velocity measurements are presented in the $\mathbf{E} \times \mathbf{B}$ direction and are compared to predictions from measured plasma potential gradients. Ion flow fields are compared to predictions from collisional fluid simulations and particle-in-cell models. These models require collisions to reproduce the ion drifts well. Neutral particle distributions are observed to be in thermal equilibrium with the chamber walls $\left(T_{n} \approx 0.028 \mathrm{eV}\right)$ and essentially non-flowing on average. Charge exchange population fractions are expected to be $\mathcal{O}\left(10^{-2}\right)$, and are too small to be resolved, but are excluded to the $5 \%$ level. 
To Little Fig 
"O me! O life!... of the questions of these recurring;

Of the endless trains of the faithless - of cities fill'd with the foolish;

Of myself forever reproaching myself, (for who more foolish than I, and who more faithless?)

Of eyes that vainly crave the light — of the objects mean — of the struggle ever renew'd;

Of the poor results of all — of the plodding and sordid crowds I see around me;

Of the empty and useless years of the rest - with the rest me intertwined;

The question, O me! so sad, recurring — What good amid these, $\mathrm{O}$ me, O life?

Answer.

That you are here - that life exists, and identity;

That the powerful play goes on, and you may contribute a verse."

Walt Whitman, Leaves of Grass (1892) 


\section{Acknowledgements}

This is my attempt at thanking in a sentence or two people who have shaped my life to this point and continue to do so. Many people have had my back, and I'm sure to miss someone. Nevertheless, here we go.

I want to first express my gratitude for the support of my advisor, Earl Scime, who smoothed my way to WVU and who for the last two and half years has trusted me with his lab probably more than he had reason to. He has answered annoying questions at 7 am and after midnight and was available for lunch almost every day I've been in Morgantown. That kind of face-to-face relationship building is exceptional and not lost on me. He is a mentor and a friend.

Enormous gratitude to Davide Curreli, Rinat Khaziev, and Shane Keniley at the University of Illinois Urbana-Champaign, and to Umair Siddiqui, with whom I've worked since early in my time at Madison. They were instrumental in laying the foundations of this project and seeing it through. In particular, I want to thank Rinat, who is always patient with my flood of questions, and Umair, whose recent work provided the inspiration for this work. Without them the project would have been something less.

I thank Amy Keesee, who brought me on as a collaborator for her work at NASA, provided much-needed expertise in argon neutral atomic physics to that component of this project, wrote a dissertation that I refer to constantly, and showed me mercy after I temporarily disabled part of her Tesla while on the highway. I would like to thank Timothy Good for his good company, humor, advice, and insightful challenges, which were greatly appreciated and enjoyed during long data campaigns. In addition to graciously agreeing to serve on my committee, Paul Cassak has been an extremely valuable resource and teacher ever since I arrived at WVU. I am fortunate to have met and worked with all of my thesis committee members.

I would like to thank Ray Fonck, Dave Smith, George McKee, and Noah Hershkowitz, my advisors at the University of Wisconsin-Madison, along with the staff and students of the Pegasus Toroidal Experiment in Madison and NSTX in Princeton. Greg Winz and Cyrus Nourgostar taught me an enormous amount that has been repeatedly useful. Out of everyone I've worked with, I have probably endangered them most, and they still always approached the work with good humor.

My undergraduate advisors - Nicolas Dauphas, Josh Frieman, Juan Estrada, Emily Nurse, and Young-Kee Kim - always supported me beyond what my early efforts probably deserved. They are all exceptional scientists whom I attempt to emulate in various ways. I see much of their guidance in the best parts of the work I'm presenting here.

J.R. Raber, Doug Mathess, and Carl Weber helped design and fabricate the intricate components of the test boundary and several diagnostics. They entertained 
literal fever dreams during this project and made it happen. Viola Bryant, Sherry Puskar, and Beena Bhanushali expertly cleared away distractions and were always patient with me.

The students whom I've worked with during my time at WVU - Mikal Dufor, Takeru Furukawa, Jacob McLaughlin, Andrew Jemiolo, Luke Neal, Xiao Zhang, Zachary Short, Julianne McIlvain, Yujian Zhao - have all contributed to this project or have in some way helped me prepare for this work. Many of them were involved in the considerable data acquisition stage. Drew Elliott never let me settle; Dave Caron props me up with his chemistry and material science insights and encyclopedic knowledge of funny things; Thomas Steinberger is an invaluable sounding board and motivator; Evan Aguirre shared HELIX/LEIA without violence and generously included me in some of his research; and John McKee had the bravery to wade into deep Langmuir probe theory with me. From them I have learned more than they know. I hope Miguel Henriquez and Mindy Bakken, with whom I've worked closely, find that they too have derived some benefit from our collaboration. I am proud to call them colleagues and friends.

The long journey through a Ph.D. is eased by good friends. I consider myself exceptionally fortunate to have been in the same place and time as Umair Siddiqui, who combines consistent high standards with creative restlessness. The same is true for Corey Henderson, who knows where and how to look. Jaime and Niall Henderson became a second family and were excellent co-navigators through shipwrecks galactic and lacustrine. Matt Michalak is an exceptional creator and fellow traveler. Adam Ernst in every space knows what's good. And I have been fortunate to have collaborated with Neil Lutz, I hope, on something like equal terms.

Sarah and Neil Peachey have always been encouraging on rough days, silly on easy days, and good company always. Dorothy and Rodney Wong have become good friends and have given me a second home. I attribute my enjoyment of knowledge and pursuit of understanding to my parents, who have encouraged blending curiosity with hard work since I can remember. They set it all in motion and deserve more credit than they can ever get.

Finally, I want to thank Marisa, my ally and accomplice. She is the kernel and the sum.

Thank you all. 


\section{Contents}

Dedication $\quad$ iii

Acknowledgements $\quad$ v

List of Figures $\quad$ x

List of Tables $\quad$ xiii

Abbreviations $\quad$ xiv

1 Motivation and context 1

1.1 The Science . . . . . . . . . . . . . . . . . . . 1

1.1.1 Weakly collisional presheaths in oblique magnetic fields . . . 3

1.2 The engineering . . . . . . . . . . . . . . . . . 4

1.3 The helicon as a plasma boundary test bed . . . . . . . . . . . . 7

1.3.1 Comparison of the HELIX helicon source to other plasmas . 7

1.3.2 Heating and plasma production in helicon plasmas . . . . 8

1.3.3 Density, temperature, and rotation profiles in HELIX . . . . 15

1.3.4 Neutral depletion in the plasma core . . . . . . . . . . . . 17

1.4 Dissertation overview . . . . . . . . . . . . . . . . . . . 19

2 Experimental Apparatus $\quad 22$

2.1 The HELIX/LEIA facility . . . . . . . . . . . . . . . . . . . 22

2.1.1 $\pi$ network matching circuit . . . . . . . . . . . . . 24

2.2 Absorbing boundary . . . . . . . . . . . . . . . . . . . . . . . . . . . . . . . . 38

2.3 Boundary coordinates . . . . . . . . . . . . . . . 30

2.3.1 Translation stage coordinates . . . . . . . . . . . . . 31

2.3.2 Simulation coordinates . . . . . . . . . . . . 33

3 Diagnostics $\quad 35$

3.1 Gaussmeter ... . . . . . . . . . . . . . . 35

3.2 Langmuir probes . . . . . . . . . . . . . . . . . . . . . . . . . . . . . . . . . . . . . . . . .

3.2.1 Langmuir probe construction . . . . . . . . . . . . 38

3.2 .2 Langmuir probe circuit . . . . . . . . . . . . . . . . . . . . . . . . . . . . . 46

3.2 .3 Langmuir probe analysis . . . . . . . . . . . . . 46 
3.2.3.1 Introduction . . . . . . . . . . . . 46

3.2.3.2 Calculation of the fluid quantities $n_{i}, n_{e}$, and $T_{e} .49$

3.2.3.3 EDFs, EEDFs and EEPFs . . . . . . . . . 57

3.2.3.4 Other considerations . . . . . . . . . . . . . 71

3.2.4 Electrostatic fluctuations . . . . . . . . . . . . . . . 74

3.3 Laser induced fluorescence . . . . . . . . . . . . . . . . . . . 75

3.3 .1 Overview .......................... 75

3.3.2 Velocity distributions from LIF data . . . . . . . . . . . . 77

3.3 .3 Optical arrangement ................ 81

3.3 .4 Conventional LIF . . . . . . . . . . . . . . . . . . . . . . . . . . . . . . . . 84

3.3 .5 Confocal LIF . . . . . . . . . . . . . . . . . . . . . 87

3.3 .6 Ar-I LIF . . . . . . . . . . . . . . . . . . . . . . . . 93

3.3 .7 Ar-II LIF . . . . . . . . . . . . . . . . . . . . . . . 98

3.3.8 Other considerations . . . . . . . . . . . . . . 99

4 Three-dimensional, multispecies measurements in a boundary with an oblique magnetic field: $B=600 \mathrm{G}, \psi=74^{\circ} \quad 106$

4.1 Introduction . . . . . . . . . . . . . . . . . . 106

4.2 Magnetic field measurements . . . . . . . . . . . . . . . . . . 107

4.3 EEPF, $n_{e}, T_{\text {eff }}$, and $p_{e}$ from Langmuir probe measurements . . . . 108

4.3.1 Non-Maxwellian features are observed in EEPFs . . . . . . . 108

4.3.2 The helicon source creates a radial density gradient . . . . . 110

4.3.3 $T_{\text {eff }}$ distribution reflects high-energy electron populations . . 112

4.3.4 $p_{e}=n_{e} k_{\mathrm{B}} T_{\mathrm{e}}$ shows a radial helicon electron pressure gradient 114

$4.4 \phi_{p}$ depends on proximity to the boundary surface . . . . . . . . . 114

4.5 Ion temperature and pressure are observed in three dimensions from

LIF distributions . . . . . . . . . . . . . . . . . . 118

4.5.1 $T_{i}$ is slightly anisotropic . . . . . . . . . . . . . . . . . . . . . . . . . . . .

4.5.2 The ion pressure is isotropic . . . . . . . . . . . . 120

4.5.3 Characteristic velocities in the boundary . . . . . . . . . 123

4.6 Characteristic frequencies in the boundary . . . . . . . . . . . . . 124

4.7 The metastable Ar-II drift velocities measured by LIF show a 3D flow field . . . . . . . . . . . . . . . . . 133

4.8 Testing Chodura's model . . . . . . . . . . . . . . . 136

4.9 Comparison of HELIX boundary data with collisional fluid and Particle-in-Cell simulations . . . . . . . . . . . . . . . . . . . 144

4.10 Ion-neutral collisions in the presheath . . . . . . . . . . . . . . . . 149

4.11 Characterizing the drift in the $\mathbf{E} \times \mathbf{B}$ direction . . . . . . . . . . . 153

5 Conclusions

$\begin{array}{lr}\text { A Digital smoothing of signals } & 159\end{array}$

A.1 Overview . . . . . . . . . . . . . . . . . . . . 159 
A.2 Moving average . . . . . . . . . . . . . . . . . 160

A.3 Gaussian convolution . . . . . . . . . . . . . . . . . . 161

A.4 Blackman window convolution . . . . . . . . . . . . . . . 162

A.5 Savitzky-Golay filtering . . . . . . . . . . . . . . . . . . . . . . . . . . . . . . . . . . . . . . . . . . .

A.6 Summary of results . . . . . . . . . . . . . . . . . 165

$\begin{array}{lr}\text { B Figure Permissions } & 168\end{array}$

$\begin{array}{ll}\text { Bibliography } & 197\end{array}$ 


\section{List of Figures}

1.1 Poloidal cross section of the ITER tokamak divertor . . . . . . . . . 5

1.2 Anomalous erosion observed in Hall thruster channels . . . . . . . . 6

1.3 Energetic electron populations observed in HELIX . . . . . . . . . . 13

$1.4 T_{e}, n$, and $T_{i}$ vs $f_{\mathrm{RF}}$ and $B$ in HELIX . . . . . . . . . . . 13

1.5 Images of the mode structure in CSDX . . . . . . . . . . . . . . 14

1.6 Radial $T_{i}$ profile . . . . . . . . . . . . . . . . . . . 15

1.7 LIF intensity profile in HELIX . . . . . . . . . . . . . . . . . . . . . . . . . . . . 16

1.8 Radial flow profiles in HELIX . . . . . . . . . . . . . . . . . . 17

1.9 Heatmap of plasma column rotation in HELIX . . . . . . . . . . . . 18

1.10 Axial flow dependence on magnetic field in CSDX . . . . . . . . . . 19

1.11 HELIX Ar-I density and ion-neutral collision rate profiles . . . . . . 19

1.12 Kr-I density profiles and dependences in CHEWIE . . . . . . . . . . 20

2.1 Overview schematic of the HELIX chamber . . . . . . . . . . . . 23

2.2 Circuit diagram: $\pi$ matching circuit . . . . . . . . . . . 25

2.3 HELIX load and tuning capacitor ranges . . . . . . . . . . . . . 27

2.4 Photograph of the (Type 316) stainless steel absorbing boundary . . 28

2.5 Photograph detail of the (Type 316 SS) laser beam dump . . . . . . 29

2.6 Photograph of the absorbing boundary immersed in the HELIX plasma . . . . . . . . . . . . . . . 30

2.7 Magnetic field perturbation from boundary . . . . . . . . . . . . . 30

2.8 Diagram of coordinate systems . . . . . . . . . . . . . . . . . . . . . . . . . . . 31

2.9 Limits of experimental domain . . . . . . . . . . . . . . . . . . . . . . . . . . 32

2.10 LIF and LP measurement locations . . . . . . . . . . . . . . . . 33

3.1 Gaussmeter magnetic field orientation . . . . . . . . . . . . . . 37

3.2 HELIX cutaway showing probe translation mechanism . . . . . . . 40

3.3 Langmuir probe electrode on translation stage . . . . . . . . . . . 40

3.4 Langmuir probe circuit diagram . . . . . . . . . . . . . . . . . . 41

3.5 Input and output frequency sweeps for Langmuir and emissive probes. 46

3.6 Bode plot for Langmuir and emissive probes . . . . . . . . . . . . . 46

3.7 Probe characteristic showing: I. ion saturation, II. transition region, III. electron saturation. . . . . . . . . . . . . . . . . . . 48

3.8 Example conventional Langmuir probe analysis . . . . . . . . . . . 52

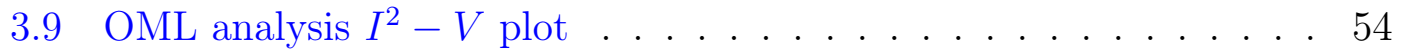

$3.10 \Psi$ range for $T_{e}=4 \mathrm{eV}$ and $B=600$ gauss . . . . . . . . . . 64 
$3.11 \Psi$ range for $r_{p}=250 \mu \mathrm{m}$ and $\ell_{p}=2 \mathrm{~mm} \ldots \ldots . . . . . . .65$

3.12 Example EEPF calculated using conventional Druyvesteyn analysis 66

3.13 Example EEPF calculated using strongly magnetized analysis . . . 66

$3.14 I_{e}^{\prime \prime}$ reconstructed using extended Druyvesteyn analysis . . . . . . . . 68

3.15 Dependence of $K^{\prime \prime}$ and $\Psi$ on relative potential. . . . . . . . . . . . 68

3.16 PSD of $\tilde{\phi}_{f} \ldots \ldots \ldots \ldots \ldots \ldots$. . . . . . . . . . . . . . . . . . . . . . . . .

3.17 Example IVDF measured in $p=3.5$ mTorr, $P_{\mathrm{RF}}=650 \mathrm{~W}, B=600$

G plasma . . . . . . . . . . . . . . . . . . . 78

3.18 Example overintegration of VDFs . . . . . . . . . . . . . . 80

3.19 Overintegration trend in mean velocity for scan time selection . . . 81

3.20 Sirah Matisse DR LIF optical arrangement . . . . . . . . . . . . . . 82

3.21 Diagram of 3D stage . . . . . . . . . . . . . . . . . . 85

3.22 Diagram of axial injection placement on HELIX . . . . . . . . . . . 86

3.23 Ray trace diagram of 3D stage optics . . . . . . . . . . . . . . . . 87

3.24 Diagram of axial injection stage . . . . . . . . . . . . . . . . . . 88

3.25 Ray trace diagram of confocal optics . . . . . . . . . . . . . . . . 89

3.26 Diagram of confocal deployment on HELIX . . . . . . . . . . . . . . 89

3.27 Ion temperature and mean flow profiles measured by conventional

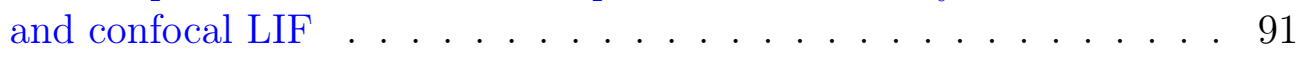

3.28 Confocal beam profiles for $f=150 \mathrm{~mm}$. . . . . . . . . . . . . . 92

3.29 Relative metastable density profiles measured by conventional and

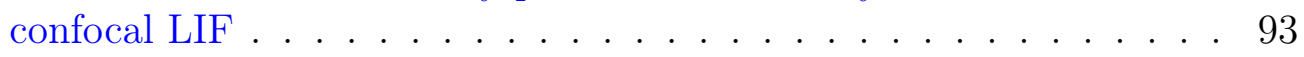

3.30 Grotrian diagram for metastable Ar-I LIF . . . . . . . . . . . . . . . 95

3.31 Typical Ar-I spectra showing Zeeman splitting . . . . . . . . . . . . . 96

3.32 Ar-I spectral width laser power dependence . . . . . . . . . . . . . . 98

3.33 Ar-I spectrum recorded parallel to the magnetic field . . . . . . . . 99

3.34 Dependence of Zeeman-split cluster separation on B . . . . . . . . 100

3.35 Transition diagram for metastable Ar-II LIF . . . . . . . . . . . . 100

3.36 IVDF series showing laser reflections . . . . . . . . . . . . . . . 101

3.37 Beam profiles for Matisse laser . . . . . . . . . . . . . . . . . 103

4.1 Detail of the coordinate systems showing the plasma shift . . . . . . 107

4.2 Hall effect probe measurements of $B_{\|}=B_{y^{*}}$. . . . . . . . . . . . 109

4.3 EEPF dependence on plasma radius and axial location . . . . . . . 110

$4.4 n_{e}(\mathbf{x})$ in the boundary region . . . . . . . . . . . . . . . 111

4.5 Electron density and temperature dependence on $z$. . . . . . . 112

$4.6 T_{\text {eff }}(\mathbf{x})$ in the boundary region . . . . . . . . . . . . . . . . 113

4.7 Spatial structure of the electron pressure, $p_{e} \ldots \ldots$. . . . . . . . . . . . 115

4.8 Electron and ion pressure dependence on $z$. . . . . . . . . . 116

4.9 Measured $\phi_{p}(\mathrm{x})$ in the boundary region . . . . . . . . . . . . . . 117

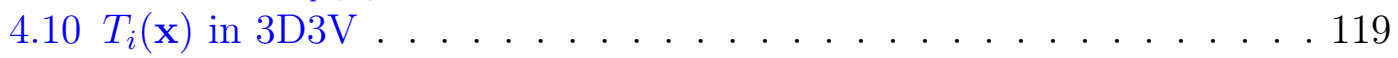

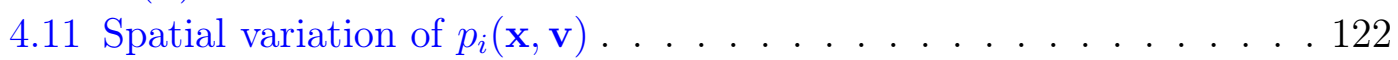

4.12 Experimental ion collision cross-sections . . . . . . . . . . . 126

4.13 Experimental $\sigma_{\mathrm{CX}}$ and $\sigma_{\mathrm{SC}}[1]$, with $\nu_{\mathrm{CX}}$ and $\nu_{\mathrm{SC}} \ldots \ldots . . . . .127$ 
4.14 Dependence of $K_{\mathrm{IZ}}$ and $\nu_{\mathrm{IZ}}$ on $n_{n}$ and $T_{e} \ldots \ldots$. . . . . . . 127

4.15 Neutral depletion on-axis in HELIX . . . . . . . . . . . . . . . . . 128

4.16 Dependence of $\ln \Lambda$ on $T_{e}, T_{i}$ and $n \ldots \ldots$. . . . . . . . . 130

4.17 Dependence of Coulomb collision frequencies on $T_{e}, T_{i}$ and $n_{e} \approx n_{i} .131$

4.18 3D map of Ar-II metastable drift velocities from LIF data . . . . . 134

4.19 Comparison between $\mathbf{u}_{i}$ measurements in MARIA and Chodura's prediction . . . . . . . . . . . . . . . 137

4.20 Comparison of predicted and observed $\lambda_{\mathrm{mps}}$. . . . . . . . . . . 137

4.21 Data showing decrease in $u_{z}$ near boundary before interpolation . . 138

$4.22 v_{z}$ distribution function cascade with distance to the boundary surface139

4.23 3D heatmap of Bohm-Chodura boundary . . . . . . . . . . . . . . . 141

4.24 3D projection of Bohm-Chodura boundary . . . . . . . . . . . . . 142

4.25 Total flow minus field-parallel flow approaching the boundary surface143

4.26 Comparison of data and simulations . . . . . . . . . . . . . . . 146

4.27 Normalized $u_{y}$ and $u_{z}$ as functions of $u_{x} \ldots \ldots$. . . . . . . . . . . . . . . . . . . . . . 147

4.28 Mean axial flow of $1 s_{5}$ metastable Ar-I . . . . . . . . . . . . . . . . 149

4.29 Large scale axial potential structure observed in the MARIA experiment . . . . . . . . . . . . . . . . 150

$4.30 u_{x}$ dependence on neutral pressure in simulations . . . . . . . . 150

4.31 Metastable Ar-I velocity distribution evolution with proximity to the wall . . . . . . . . . . . . . . . . . . 151

4.32 Comparison of Ar-I and Ar-II spectra at close proximity to the wall 152

$4.33 u_{z}$ for three fill pressures . . . . . . . . . . . . . . . . . 153

$4.34 u_{z}$ for three fill pressures . . . . . . . . . . . . . . . 154

A.1 Comparison of probe characteristics smoothed by moving average . 161

A.2 Comparison of window widths used in moving average smoothing . 161

A.3 Comparison of Gaussian smoothed probe characteristics . . . . . . . 162

A.4 Comparison of several $\sigma$ used in Gaussian smoothing . . . . . . . . 163

A.5 Comparison of probe characteristics smoothed by Blackman filtering 163

A.6 Comparison of window widths used for Blackman filtering . . . . . 164

A.7 Comparison of probe characteristics smoothed by Savitzky-Golay filtering . . . . . . . . . . . . . . . 165

A.8 Comparison of window widths used for Savitzky-Golay filtering . . . 165 


\section{List of Tables}

1.1 Comparison of plasma quantities: DIII-D SOL, BHT-600 Hall thruster,

HELIX .......................... 7

2.1 HELIX facility engineering parameters . . . . . . . . . . . . . . 22

2.2 HELIX facility plasma quantities . . . . . . . . . . . . . . . 24

3.1 RF choke specifications used in Langmuir probe compensation circuit 44

3.2 DS345 settings for electrostatic probe frequency response measurements .......................... 45

3.3 Scale lengths in a sample helicon plasma in HELIX . . . . . . . . . 61

3.4 Summary of the Langmuir probe results for a sample characteristic 69

3.5 HELIX chamber dimensions and typical plasma conditions . . . . . 73

3.6 Summary of Ar-I metastable $4 s\left[{ }^{2} P_{3 / 2}^{0}\right]_{2}\left(1 s_{5}\right)$ LIF schemes with VIS/NIR transitions . . . . . . . . . . . . . . . . 94

3.7 Summary of Ar-I metastable $4 s\left[{ }^{2} P_{3 / 2}^{0}\right]_{2}\left(1 s_{5}\right)$ Zeeman effect . . . . . 97

3.8 Approximate transmission coefficients of elements in the LIF optical

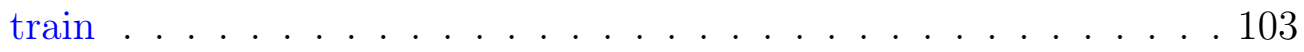

3.9 Response function parameters . . . . . . . . . . . . . . . 104

4.1 Fiducial measurements for $\delta T_{i}$ (stat.) . . . . . . . . . . . . . . . . 121

4.2 Characteristic velocities . . . . . . . . . . . . . . . . 124

4.3 Plasma and cyclotron frequencies for both charged species . . . . . 124

4.4 Collision frequencies for measured range of $T_{e}, T_{i}$, and $n \ldots 132$

A.1 Summary of digital smoothing techniques . . . . . . . . . . . 166 


\section{Abbreviations}

3D Three orthogonal spatial dimensions

3V Three orthogonal velocity dimensions

ABR Allen, Boyd, and Reynolds

APC Angled physical contact (fiber connector)

Ar-I Neutral argon gas

Ar-II Single-ionized argon

AWG American wire gauge

BHT Busek Hall effect Thruster

BNC Bayonet Neill-Concelman (connector/standard)

BRL Bernstein, Rabinowitz, and Laframboise

CCD Charge-coupled device

CL Child-Langmuir

CR Collisional-Radiative model

CSDX Controlled Shear Decorrelation Experiment

CSV Comma separated variable

CX Charge exchange

DR Dye ring

EDF Electron distribution function

EEDF Electron energy distribution function

EEPF Electron energy probability function

EG Ethylene glycol

EI Electron-ion Coulomb scattering

EMI Electromagnetic interference

EP Emissive probe 
EPH Ethylene glycol monophenyl ether

FC Ceramic ferrule (fiber connector)

FWHM Full-width at half-maximum

GaAs Gallium arsenide

HELIX Hot hELIcon eXperiment

ID Inner diameter

IE Ion-electron Coulomb scattering

II Ion-ion Coulomb scattering

InAs Indium arsenide

ITER International Thermonuclear Experimental Reactor

IV Current-voltage (trace)

IVDF Neutral velocity distribution function

IZ Ionization

Kr-I Neutral krypton gas

Kr-II Singly-ionized krypton

LASER Light Amplification by Stimulated Emission of Radiation

LEIA Large Experiment on Instabilities and Anisotropies

LIF Laser induced fluorescence

LP Langmuir probe

MARIA Magnetized AnisotRopic Ion-distribution Apparatus

MCC Monte-Carlo collisions

MM Multimode (fiber)

MPEX Material Plasma Exposure eXperiment

MPS Magnetic presheath

NIST National Institute of Standards and Technology

NSTX National Spherical Torus Experiment

NVDF Ion velocity distribution function

OD Outer diameter

OML Orbital motion limited

PEEK Polyether ether ketone

PIC Particle-in-Cell 
PMT Photo-multiplier tube

PPPL Princeton Plasma Physics Laboratory

PSD Power spectral density

PTFE Polytetrafluoroethylene

RAID Resonant Antenna Ion Device

RDW Resistive drift wave

RF Radio-frequency

RFEA Retarding field energy analyzer

RMS Root-mean squared

RMSE Root-mean squared error

SC Elastic scattering

SM Single mode (fiber)

SMA SubMiniature version A

SNR Signal-to-Noise Ratio

SOL Scrape-off layer

TALIF Two photon absorption laser induced fluorescence

UIUC University of Illinois at Urbana-Champaign

VASIMR Variable Specific Impulse Magnetoplasma Rocket

VDF Velocity distribution function

WVU West Virginia University 


\section{Chapter 1}

\section{Motivation and context}

\subsection{The Science}

The importance of the boundary region to the total plasma discharge has been recognized nearly as long as plasmas have been studied. Early plasma physicists reasoned that the long range electromagnetic forces in almost all plasmas would eventually contact an interface, creating a boundary region termed a "sheath," and that this boundary region, though small, would impact the global plasma equilibrium [2]. As Hershkowitz succinctly argued: "sheaths matter" [3]. The physics of plasma boundaries is interesting, because advances in understanding assist technology development, as discussed in Sec. 1.2, and because sheaths and sheath-like structures occur in natural plasma phenomena as far ranging as lightning [4] and planetary plasma sheets [5].

Early plasma experiments by Langmuir and Bohm demonstrated that in unmagnetized systems the particles passing through the boundary would experience several regions. In the bulk plasma, far from the boundary, any local electric fields created by boundaries or other distant causes are shorted out by the plasma's high electrical conductivity. At the surface or just above it, the higher electron flux at least when $T_{e}>T_{i}$, for electron and ion temperatures respectively — charges the surface to a negative potential relative to the bulk plasma, and the sheath 
ceases to be quasineutral. The potential structure in the sheath repels additional low-energy electrons and attracts ions, until the ion and electron fluxes settle into an equilibrium. The constant fluxes through a stable sheath require the ion velocity toward the wall to become supersonic, i.e., $v \geq c_{s} \doteq \sqrt{k_{\mathrm{B}}\left(\gamma_{i} T_{i}+\gamma_{e} T_{e}\right) / m_{i}}$, a condition that has become known as the Bohm criterion [6]. If ions in the bulk are not moving on average and are supersonic in the sheath, then a region, termed the "presheath," is required in which they accelerate. Within the presheath, the potential gradient is gradual over a long distance and quasineutrality still holds. Significant progress has been made toward understanding sheath and presheath behavior, extending simple boundary conditions to plasmas with multiple ion species [7], biased electrodes [8], and near small or large electrodes with respect to the chamber diameter [9], to name a very small subset.

A restructuring of the boundary region occurs with the introduction of a magnetic field. In highly magnetized plasmas, where the gyro-frequency exceeds the collision rate, particles in the bulk plasma tend to follow magnetic field lines. The geometry becomes highly anisotropic, with transport along field lines dominating transport across field lines. In the opposite extreme, where the magnetic field is very weak, the plasma conditions revert to the magnetic field-less case.

For intermediate fields, particles transition from a region where the magnetic field dominates, far from the boundary, to one in which they interact with both the magnetic field and strong gradients in the plasma potential. These fields interact to produce nonlinear behavior and are further complicated by the host of collisions that arise as particles from the plasma and particles at the interface intermingle. These effects conspire to create a plasma that is three dimensional and potentially non-Maxwellian. Theoretical and computational investigations that attempt to replicate particle motion in these boundaries are challenging. Models must describe the gradual spatial variations of the presheath and the sharp gradients of the sheath; the long time-scale behavior such as ion or neutral thermal drifts and fast electron behavior; and often the non-thermal distribution functions that result from inelastic collisions. All of these features require additional computational expense, and omitting some of them in the interest of computational expediency can 
easily result in inaccuracies. Determining which features should be neglected and which ones are essential is one aim of using experimental observations to validate codes.

\subsubsection{Weakly collisional presheaths in oblique magnetic fields}

Chodura [10] is typically credited with initiating the study of presheaths in oblique magnetic fields, when the angle $\psi$ between the normal vector of the interface and the magnetic field lines is nearly $90^{\circ}$. In this model, the plasma behaves like a fluid and only the spatial and drift velocity components along the surface normal are resolved. Collisions are neglected, as are finite gyro-radius effects, and the ions are assumed to follow an isotropic Maxwellian distribution. Chodura proposed a new region of the boundary, which he called the "magnetic presheath," in which the plasma was still quasi-neutral, but where the electric field of the boundary is sufficient to cause particles to deflect from their magnetic field-parallel trajectories. In this "Chodura layer," the ions are supersonic parallel to the magnetic field, but are not yet supersonic normal to the wall. A classical "plasma presheath" accelerates the ions to the $u_{\|} \geq c_{s}$ condition. The boundary of the Chodura layer is established by a modified criterion, referred to as the Bohm-Chodura criterion, which states that the ion flow toward the boundary must become supersonic, but reduced according to the magnetic angle:

$$
1>u_{x} / c_{s} \geq \cos \psi
$$

He predicted that this condition would be satisfied at a distance

$$
\lambda_{\mathrm{mps}}=\sqrt{6}\left(c_{s} / \omega_{c i}\right) \sin \psi
$$

from the Debye sheath edge. In the limit that the magnetic field and the surface normal are aligned, $\psi \rightarrow 0$, the Chodura layer vanishes, and the Bohm criterion is recovered. 
Since that time, magnetic presheath research has enjoyed broad theoretical progress [11-17]. Only a few experiments exist in literature to provide data to validate these models [18-23]. Of these experiments, the early focus was on mapping the plasma potential structure of the boundary region with electrostatic probes. Siddiqui et al. were the first to report measurements of argon ion distribution functions and 2D flow fields in a boundary with $\psi \leq 60^{\circ}$ [22], which they compared to a collisional fluid model. Preliminary results related to the experiments reported in this dissertation were described two years later in Ref. [23]. Early measurements of ion drifts in the direction parallel to $\mathbf{E} \times \mathbf{B}$ were reported for the first time.

Measurements of the background metastable neutral population were unavailable to the authors of Refs. [22] and [23]. Through elastic scattering collisions, ionization, and charge exchange, ion and neutral populations modify each other's distribution functions. Collisionality depends on the relative drifts of the two populations, and even a coarse neutral flow measurement is useful for constraining collisional models. More detailed distribution measurements provide velocity distribution data on the neutral population and reveal the presence of any nonMaxwellian collisional markers such as charge exchange [24]. Metastable neutrals, especially the $1 s_{5}$ state described in more detail in Sec. 3.3.6 and in [25], make up a large fraction of the total neutral population and have long state lifetimes, making them advantageous for measuring the transient conditions of the boundary.

\subsection{The engineering}

The implications of magnetic presheath physics extend into engineering and technological applications. Sheaths in magnetic environments appear around spacecraft antennae [26] and terrestrial diagnostic probes [27]. Magnetic confinement fusion devices, such as tokamaks, use strong magnetic fields to divert particles and heat to specific regions of the chamber wall called divertors [28]. Fig. 1.1 shows an example poloidal cross section of the proposed ITER tokamak with magnetic field lines contacting the wall at oblique angles in the divertor region. In this 


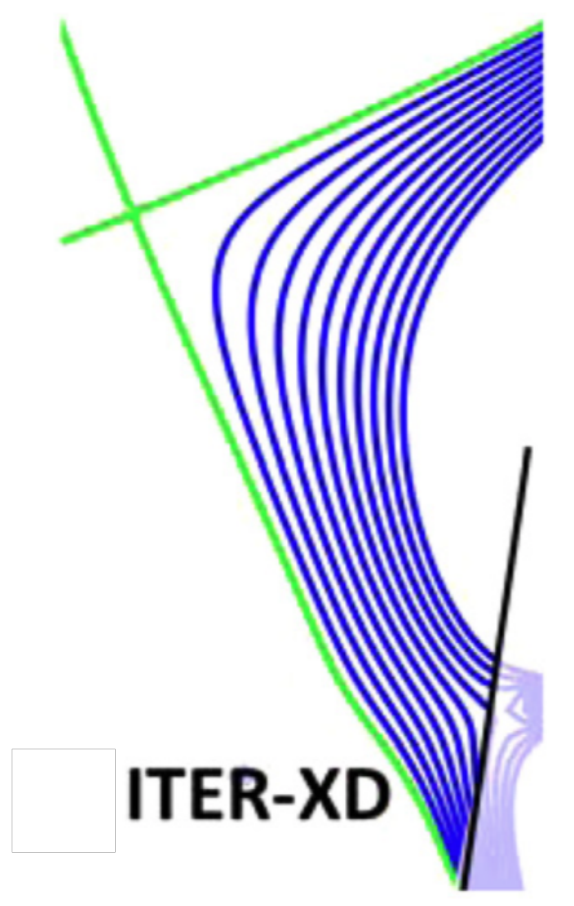

Figure 1.1: Poloidal cross section of the ITER tokamak divertor region [28]. Blue lines indicate magnetic flux surfaces. Green lines mark X-point and the separatrix. Black line indicates chamber wall surface. Reprinted from $M$. Kotschenreuther, P. Valanju, B. Covele, S. Mahajan, Physics of Plasmas, 20, 102507 (2013), with the permission of AIP Publishing.

region, careful shaping of the magnetic field distributes the extreme particle and heat loads over a large area to reduce concentrations of particle flux and power. Particle and heat fluxes modify the surface and the plasma in several ways. Sputtering and erosion caused by particle bombardment change the shape of the wall over time, and depend, among other things, on the ion energy, flux, and impact angle [29]. In the process, some of the wall material returns to the plasma. Wall material is of higher mass and atomic number $Z$ relative to the plasma - walls are often carbon $\left(Z_{C}=6\right)$, molybdenum $\left(Z_{M o}=42\right)$, or tungsten $\left(Z_{W}=74\right)$, compared to the hydrogen $\left(Z_{H}=1\right)$ or helium $\left(Z_{H e}=2\right)$ plasma - and because these elements are more easily ionized, power losses from the lighter species occur. Gas particles trapped in the lattice of the wall material are released, affecting gas fueling control and plasma shaping in the edge. Heat and particle fluxes are determined in part by the boundary regions that separate the chamber wall from the hot bulk plasma $[16,29,30]$. 

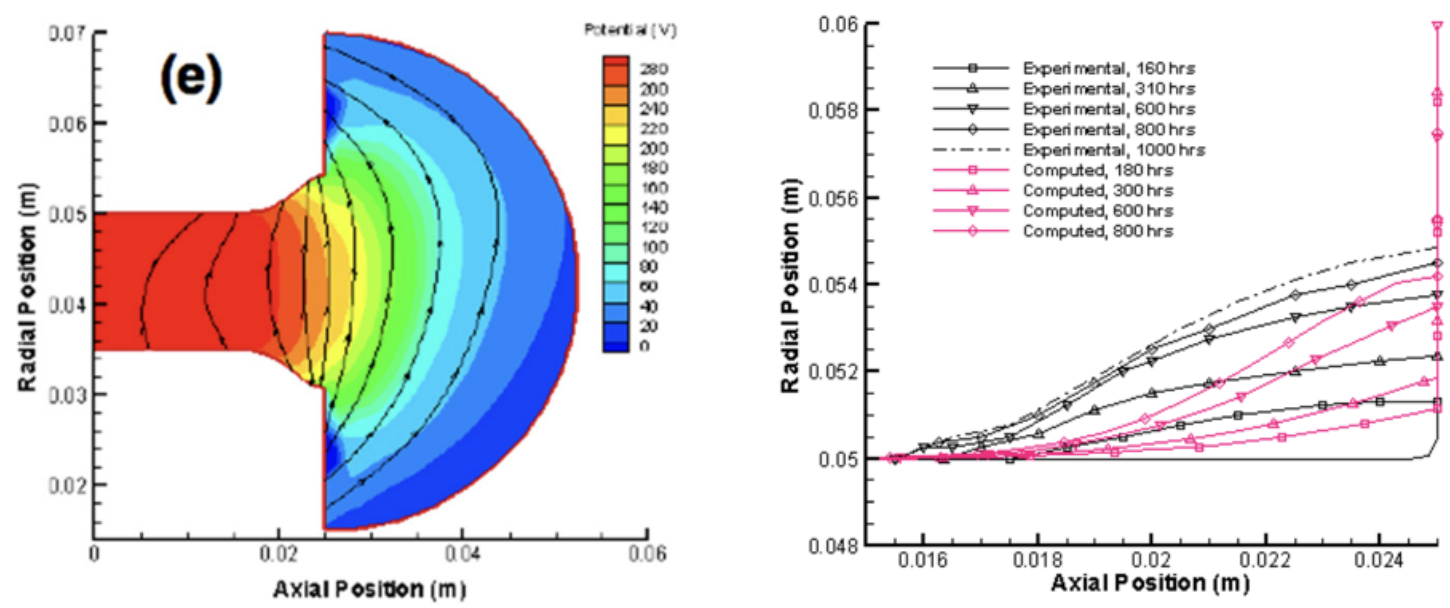

Figure 1.2: Anomalous erosion observed in Hall thruster channels during lifetime tests [23]. Reprinted from M. U. Siddiqui, D. S. Thompson, C. D. Jackson, J. F. Kim, and N. Hershkowitz, Physics of Plasmas, 23, 057101 (2016), with the permission of AIP Publishing. Original images courtesy of $M$. Gamero-Castaño and I. Katz [31].

Recently, Siddiqui et al. argued that magnetic presheath physics might explain anomalous erosion rates observed in Hall thruster channels (see Fig. 1.2 [23]). Hall thruster channel erosion sets up the conditions for the main failure mode of Hall thrusters [32], and reducing it is critical for extending thruster operational lifetimes. Recent order-of-magnitude lifetime extensions have been achieved in magnetically shielded Hall thrusters [32, 33]. This extension is attributed to a modification of the potential structure by channel and magnetic field shaping, which effectively reduces the flux and impact energy of ions at the channel wall. It is worth mentioning that the popular Hall thruster modeling Particle-in-Cell code HPHall does not include ion drifts in the $\mathbf{E} \times \mathbf{B}$ direction, which Siddiqui et al. argue may account for discrepancies between Hall thruster simulations and experiments [23]. 


\begin{tabular}{lccc}
\hline \hline $\begin{array}{l}\text { Plasma } \\
\text { parameter }\end{array}$ & $\begin{array}{c}\text { DIII-D detached } \\
\text { SOL }\end{array}$ & $\begin{array}{c}\text { BHT-600 Hall } \\
\text { thruster }\end{array}$ & $\begin{array}{c}\text { Helicon } \\
\text { plasma }\end{array}$ \\
\hline$n_{o}\left(\mathrm{~m}^{-3}\right)$ & $10^{19}-10^{20}$ & $10^{17}-10^{18}$ & $10^{18}-10^{19}$ \\
$n_{n}\left(\mathrm{~m}^{-3}\right)$ & $10^{18}-10^{20}$ & $10^{17}-10^{20}$ & $10^{19}-10^{20}$ \\
$T_{e}(\mathrm{eV})$ & $1-20$ & $1-20$ & $3-10$ \\
$T_{i}(\mathrm{eV})$ & $\sim T_{e}$ & $<0.1$ & $<1$ \\
$B(\mathrm{kG})$ & $1-10$ & $0.1-0.6$ & $0-1$ \\
$\lambda_{D}(\mathrm{~m})$ & $\approx 10^{-5}$ & $\approx 10^{-5}$ & $\approx 10^{-5}$ \\
$\lambda(\mathrm{m})$ & $\approx 0.005$ & $\approx 0.005$ & $\approx 0.005$ \\
$\rho_{i}(\mathrm{~m})$ & $<0.01$ & $0.005-\infty$ & $\approx 0.005$ \\
\hline \hline
\end{tabular}

TABLE 1.1: Comparison of plasma quantities: DIII-D SOL, BHT-600 Hall thruster, HELIX [23]. Reprinted from M. U. Siddiqui, D. S. Thompson, C. D. Jackson, J. F. Kim, and N. Hershkowitz, Physics of Plasmas, 23, 057101 (2016), with the permission of AIP Publishing. Original data from [34] and [35].

\subsection{The helicon as a plasma boundary test bed}

\subsubsection{Comparison of the HELIX helicon source to other plasmas}

As a plasma source, helicons have a high degree of variability, operate in capacitive, inductive, and helicon modes, and produce plasma densities over a range from approximately $10^{15}-10^{19} \mathrm{~m}^{-3}$. While the mechanism is a subject of on-going experiment, the high efficiency of helicon plasma production is well established and allows helicon sources to create plasmas with densities that approach the densities in tokamak divertors using only a few hundred watts. Tab. 1.1 shows a comparison of plasma parameters between the DIII-D scrape-off-layer, the BHT600 Hall thruster, and HELIX.

The magnetic fields in HELIX are on the same order as the representative BHT600 Hall thruster and at the lower end of the range for the high aspect ratio DIII-D scrape-off-layer. Low aspect ratio spherical tokamaks have magnetic fields on the order of the fields in HELIX [36-38]. The primary difference between the devices is the ion temperature, which in tokamaks can be comparable to the electron 
temperature. The temperature and field effects partially cancel in setting the ion gyro-radius in HELIX, which is therefore slightly smaller than in the tokamak SOL.

Several high-power helicons are currently in operation or development. These devices have wide-ranging applications, from interplanetary spacecraft propulsion (VASIMR [39]) to material testing and beam generation for fusion devices (ProtoMPEX [40]; RAID [41]). These devices will build on our understanding of magnetized plasma boundaries and extend them to new high-powered regimes.

\subsubsection{Heating and plasma production in helicon plasmas}

\section{A history of the helicon wave}

The helicon discharge was first named in the early 1960s by Aigrain, who was studying wave propagation in indium [42, and references therein]. Early theoretical work was pioneered by Legéndy [43], Formato and Gilardini [44], and Klozenberg, McNamara and Thonemann [45]. Reviews of the early development of helicons can be found in Ref. [46]. The study of the family of low-frequency $\left(\omega \ll \omega_{\text {pe }}\right)$ waves to which helicon waves belong, called whistler waves, is historically interesting, as it involves eavesdropping on poorly secured communications during World War I. Interpretations of whistler wave signals, which were initially observed at audible frequencies, varied from "the grenades fly" to Martian communications [46]. The whistler wave phenomenon was later ascribed to the propagation of charged particles along Earth's magnetic field lines following lightning strikes [47].

The helicon wave was observed in a toroidal plasma in 1960 [48]. Since that time, it has been developed for use at a range of frequencies [49], antenna shapes [50, 51, and references therein], and RF powers [52-55], and applied to plasma processing [56], spacecraft propulsion [57, 58], and laboratory science experiments [59]. Recent developments are reviewed in Refs. [60-62]. 


\section{The helicon dispersion relation}

The helicon is a low-frequency, radially bounded whistler wave propagating in a magnetic field. (The discussion in this section follows $[60,63,64]$ with background support from $[65,66]$.) It is useful to describe each physical quantity as the sum of a background component and a perturbation component, such that $n=n_{\mathrm{o}}+\delta n$, $\mathbf{E}=\mathbf{E}_{\mathrm{o}}+\delta \mathbf{E}$, etc., with the assumption that the perturbed quantity is much smaller than the background. The most significant behavior is described by the $0^{\text {th }}$ and $1^{\text {st }}$ order quantities, so that, e.g., $\delta n \delta v \sim 0$. The background magnetic fields are assumed to be static, have magnitude $B_{\mathrm{o}}$ and direction $\hat{z}$, and [66]:

$$
\nabla n_{\mathrm{o}}=\mathbf{v}_{\mathrm{o}}=0 \quad \frac{\partial n_{\mathrm{o}}}{\partial t}=\frac{\partial \mathbf{v}_{\mathrm{o}}}{\partial t}=\frac{\partial \mathbf{E}_{\mathrm{o}}}{\partial t}=\frac{\partial \mathbf{B}_{\mathrm{o}}}{\partial t}
$$

where $n\left[\mathrm{~m}^{-3}\right]$ is the density, $\mathbf{E}[\mathrm{V} / \mathrm{m}]$ the electric field, $\mathbf{v}[\mathrm{m} / \mathrm{s}]$ is the velocity $(v=|\mathbf{v}|), \mathbf{B}[\mathrm{T}]$ is the magnetic field, $\mathbf{J}\left[\mathrm{A} / \mathrm{m}^{2}\right]$ the current density, and $t$ is the time variable. Quasineutrality dictates that $n_{e o} \approx n_{i o}$, and in the cold plasma approximation (electron and ion temperatures follow $T_{e} \sim T_{i} \sim 0$ ) $\delta n_{i} \approx 0$. The wave is assumed to be a plane wave. Each perturbed quantity is assumed to be sinusoidal in space and time $(\mathbf{r}, t)$, with frequency $\omega$ and wavenumber $\mathbf{k} \propto 2 \pi / \lambda$, so that $\delta \mathbf{E}=\delta \mathbf{E}_{\mathrm{o}} \exp (i \mathbf{k} \cdot \mathbf{r}-\omega t)$, etc. In this framework, the linearized set of Maxwell's equations are

$$
\begin{array}{cc}
\nabla \cdot \delta \mathbf{E}=e \delta n_{e} / \epsilon_{\mathrm{o}} & \nabla \cdot \delta \mathbf{B}=0 \\
\nabla \times \delta \mathbf{E}=-\frac{\partial \delta \mathbf{B}}{\partial t} & \nabla \times \delta \mathbf{B}=\mu_{\mathrm{o}} \delta \mathbf{J}+\epsilon_{\mathrm{o}} \mu_{\mathrm{o}} \frac{\partial \delta \mathbf{E}}{\partial t}
\end{array}
$$

in SI units. From now on, all ordering subscripts will be dropped and all quantities will be assumed to be perturbations unless otherwise indicated. Using Ohm's law with a conductivity tensor $\boldsymbol{\sigma}$, i.e., without assuming that the current density is directed along $\mathbf{E}, \mathbf{J}=\boldsymbol{\sigma} \cdot \mathbf{E}$, and Ampère's law in Eq. (1.1) is

$$
\begin{aligned}
\nabla \times \mathbf{B} & =\mu_{\mathrm{o}}\left(\boldsymbol{\sigma} \cdot \mathbf{E}+\epsilon_{\mathrm{o}} \frac{\partial \mathbf{E}}{\partial t}\right)=\mu_{\mathrm{o}}\left(\boldsymbol{\sigma} \cdot \mathbf{E}-i \omega \epsilon_{\mathrm{o}} \mathbf{E}\right) \\
& =-i \frac{\omega}{c^{2}}\left(\mathbf{I}-\frac{\boldsymbol{\sigma}}{i \omega \epsilon_{\mathrm{o}}}\right) \cdot \mathbf{E} .
\end{aligned}
$$


I is the identity tensor. The quantity in parentheses is the effective dielectric tensor, $\boldsymbol{\epsilon}_{\mathrm{R}}$ (in some places $\mathbf{K}$ ), where the dielectric tensor $\boldsymbol{\epsilon}=\epsilon_{\mathrm{o}} \boldsymbol{\epsilon}_{\mathrm{R}}$. The curl of Faraday's law is

$$
\begin{aligned}
& \nabla \times(\nabla \times \mathbf{E})=\nabla \times\left(\frac{-\partial \mathbf{B}}{\partial t}\right)=-\frac{\partial}{\partial t}[\nabla \times \mathbf{B}] \\
& \nabla(\nabla \cdot \mathbf{E})-\nabla^{2} \mathbf{E}=-\frac{\partial}{\partial t}\left[-i \frac{\omega}{c^{2}}\left(\mathbf{I}-\frac{\boldsymbol{\sigma}}{i \omega \epsilon_{\mathrm{o}}}\right) \cdot \mathbf{E}\right] \\
&-\mathbf{k}(\mathbf{k} \cdot \mathbf{E})+k^{2} \mathbf{E}=\frac{\omega^{2}}{c^{2}} \boldsymbol{\epsilon}_{\mathrm{R}} \cdot \mathbf{E} . \\
& {\left[\frac{k^{2} c^{2}}{\omega^{2}}\left(\frac{\mathbf{k k}}{k^{2}}-\mathbf{I}\right)+\boldsymbol{\epsilon}_{\mathrm{R}}(\omega, \mathbf{k})\right] \cdot \mathbf{E}=0 . }
\end{aligned}
$$

i.e., [67, Eq. 9.55]

$$
\operatorname{det}\left|\frac{k^{2} c^{2}}{\omega^{2}}\left(\frac{\mathbf{k k}}{k^{2}}-\mathbf{I}\right)+\boldsymbol{\epsilon}_{\mathrm{R}}(\omega, \mathbf{k})\right|=0
$$

For a single ion argon plasma [65],

$$
\begin{array}{cc}
S=1+\frac{\omega_{\mathrm{pi}}^{2} / \omega_{\mathrm{ci}}^{2}}{1-\omega^{2} / \omega_{\mathrm{ci}}^{2}}+\frac{\omega_{\mathrm{pe}}^{2} / \omega_{\mathrm{ce}}^{2}}{1-\omega^{2} / \omega_{\mathrm{ce}}^{2}} & D=\frac{\omega_{\mathrm{ci}} \omega_{\mathrm{pi}}^{2}}{\omega\left(\omega^{2}-\omega_{\mathrm{ci}}^{2}\right)}-\frac{\omega_{\mathrm{ce}} \omega_{\mathrm{pe}}^{2}}{\omega\left(\omega^{2}-\omega_{\mathrm{ce}}^{2}\right)} \\
P \equiv 1-\frac{\omega_{\mathrm{pi}}^{2}}{\omega^{2}}-\frac{\omega_{\mathrm{pe}}^{2}}{\omega^{2}} & \\
R=S+D \quad L=S-D .
\end{array}
$$

In the cold plasma approximation, this condition simplifies to [65]

$$
\operatorname{det}\left|\begin{array}{ccc}
S-n^{2} \cos ^{2} \theta & -i D & n^{2} \cos \theta \sin \theta \\
i D & S-n^{2} & 0 \\
n^{2} \cos \theta \sin \theta & 0 & P-n^{2} \sin ^{2} \theta
\end{array}\right|=0
$$


which results in the cold plasma dispersion relation

$$
A n^{4}-B n^{2}+C=0
$$

where

$$
\begin{aligned}
A & =S \sin ^{2} \theta+P \cos ^{2} \theta \\
B & =R L \sin ^{2} \theta+P S\left(1+\cos ^{2} \theta\right) \\
C & =P R L \\
n^{2} & =k^{2} c^{2} / \omega^{2} .
\end{aligned}
$$

If the background magnetic field is taken to be aligned with $\hat{z}$, the axis of the machine, so that $\mathbf{B}=B_{\mathrm{o}} \hat{z}$, then $\theta$ is defined by the perpendicular, parallel, and total wavenumbers:

$$
\theta \equiv \arccos \left(k_{\|} / k\right)
$$

The solutions to Eq. (1.4) are [65]

$$
\tan ^{2} \theta=\frac{P\left(n^{2}-R\right)\left(n^{2}-L\right)}{\left(S n^{2}-R L\right)\left(n^{2}-P\right)} .
$$

For propagation along the background field, $k \rightarrow k_{\|}, \theta \rightarrow 0$, either $P=0$, in which only plasma oscillations exist; or $n^{2}=R$, and propagation occurs with right-handed polarization when looking parallel to $\mathbf{B}$; or $n^{2}=L$, and propagation occurs with left-handed polarization. Helicon waves form a subset of the righthanded polarization case, which for a single ion argon plasma have [65]

$$
n_{\mathrm{R}}^{2}=\frac{k^{2} c^{2}}{\omega^{2}}=\frac{\omega_{\mathrm{pe}}^{2}}{\omega_{\mathrm{ce}} \omega\left(1+\omega_{\mathrm{ci}} / \omega-\omega / \omega_{\mathrm{ce}}\right)} .
$$

For helicons with ion cyclotron frequency $\omega_{\text {ci }}$, electron cyclotron frequency $\omega_{\text {ce }}$, and electron plasma frequency $\omega_{\text {pe }}, \omega_{\text {ci }} \ll \omega \ll \omega_{\text {ce }} \ll \omega_{\text {pe }}$, and Eq. (1.5) becomes

$$
\frac{k^{2} c^{2}}{\omega^{2}}=\frac{\omega_{\mathrm{pe}}^{2}}{\omega_{\mathrm{ce}} \omega} .
$$


In physical quantities,

$$
n_{e}=\frac{k^{2}}{\omega} \frac{B}{e \mu_{o}}
$$

and the wave propagates with wavelength $\lambda[\mathrm{m}]=5.6 \times 10^{12}\left(B / n_{e} f_{\mathrm{RF}}\right)^{1 / 2}[68]$, with wave frequency, $f=\omega / 2 \pi$, in Hz. Eq. (1.7) indicates a linear dependence on the magnetic field magnitude and frequency [69]. In experiments, the density has been shown to follow this prediction; however helicons exhibit distinct mode transitions in which the density increases sharply at a threshold field magnitude [68].

\section{Progress in understanding helicon source heating and efficiency}

(This subsection borrows from the discussion in [61].) As early as the 1960s scientists began to suspect that something was missing in the early description of the efficient energy transfer between the helicon wave and the plasma. The heating could not be entirely attributed to collisional damping of the helicon waves or to Landau damping of energetic electrons [70]. At the same time, axially streaming energetic electron populations have been observed (Fig. 1.3 [71]). The ability to vary $R F$ frequency in helicon experiments has opened the possibility of testing the frequency-dependence of helicon heating. In parts (a) and (c) of Fig. 1.4, the lower hybrid frequency $\omega_{\mathrm{LH}}$ sets a clear threshold for an increase in $T_{e}$. In part (b), the maximum densities are observed at $\omega_{\mathrm{LH}}$ and generally increase for increasing $\omega_{\mathrm{LH}}$. These observations clearly show a dependence on $\omega_{\mathrm{LH}}$ which is not a part of the helicon dispersion relation in Eq. (1.6). The challenge is to reconcile two phenomena: 1) In many sources, the majority of the RF power transfer appears directly under the forward edge of the antenna, indicating an effect local to the antenna; however 2) "blue core" helicons have been observed in equilibrium several meters downstream of the antenna, suggesting electromagnetic energy propagation along the plasma column. 


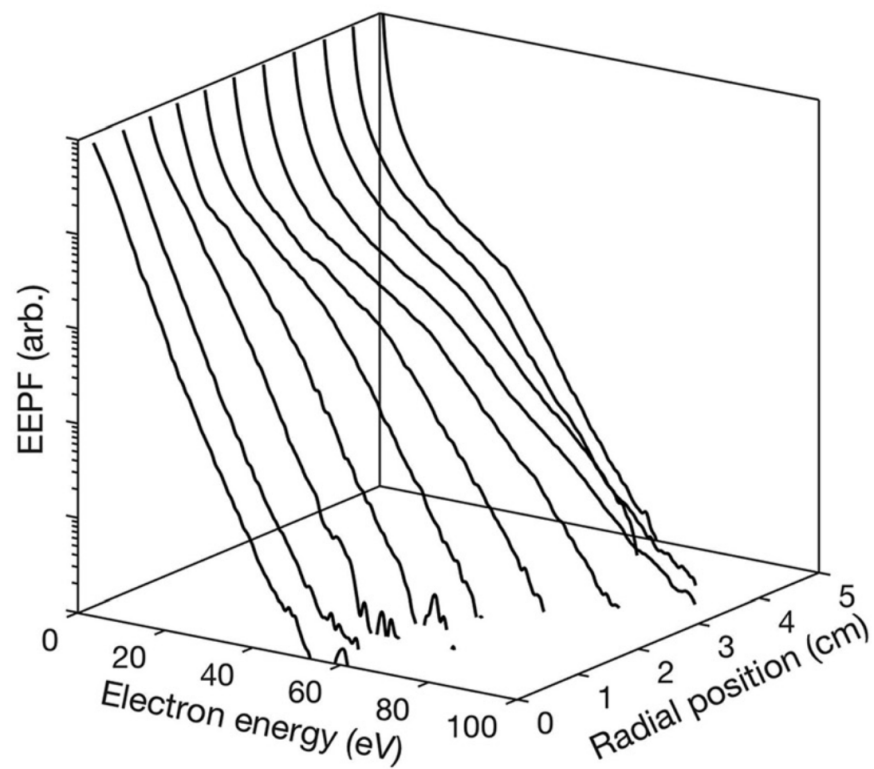

FIGURE 1.3: Energetic electron populations observed in HELIX [71].

Reprinted from E. M. Aguirre, E. E. Scime, D. S. Thompson, and T. N. Good, Physics of Plasmas 24, 123510 (2017), with the permission of AIP Publishing.
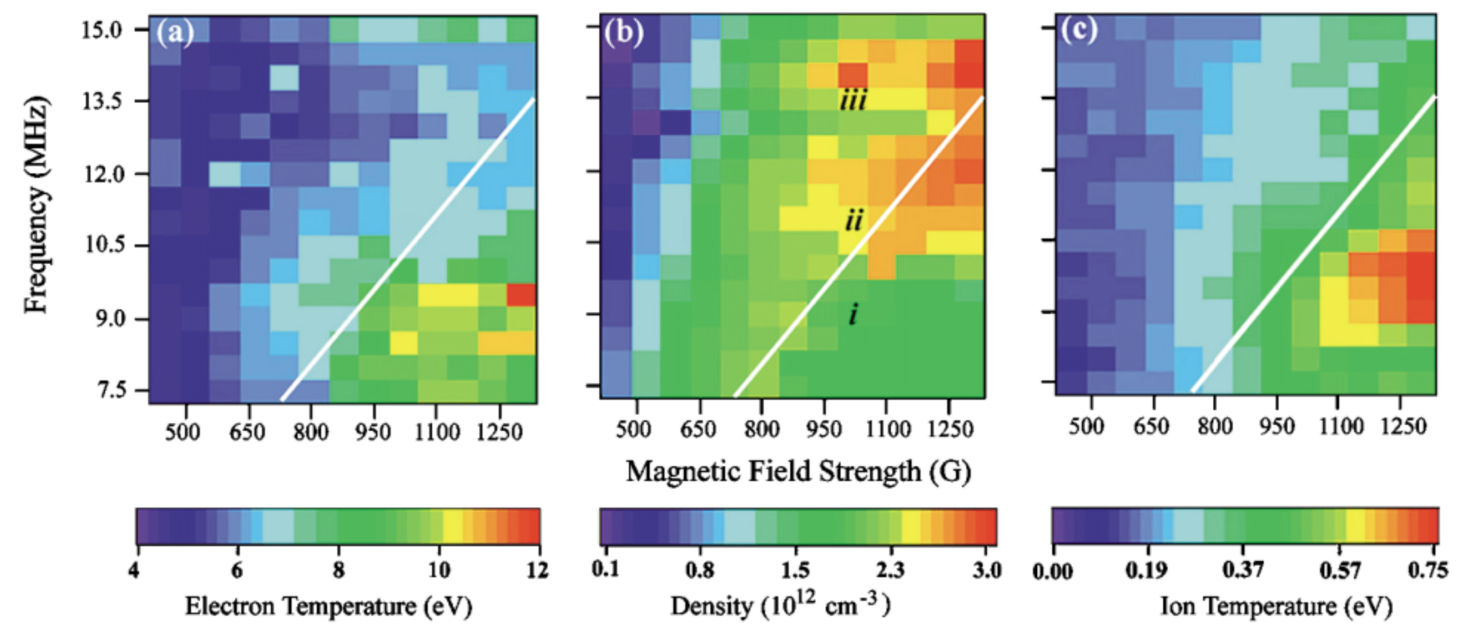

Figure 1.4: $T_{e}, n$, and $T_{i}$ vs $f_{\mathrm{RF}}$ and $B$ in HELIX [72]. The white line marks the on-axis lower hybrid frequency. Reprinted Fig. 5 with permission from $J$. L. Kline, E. E. Scime, R. F. Boivin, A. M. Keesee, and X. Sun, and V. S. Mikhailenko, Physical Review Letters, 88, 195002 (2002). Copyright (2002) by the American Physical Society.

The underlying physics depends, in an as-yet incompletely understood way, on the radial geometry and transport of the plasma and on the axial boundary conditions. In recent experiments, low-frequency resistive drift wave (RDW) instabilities in regions of strong radial density gradients separate two other plasma regions: 1) a bright core with a rotating mode and 2) a turbulent edge. Radial transport 


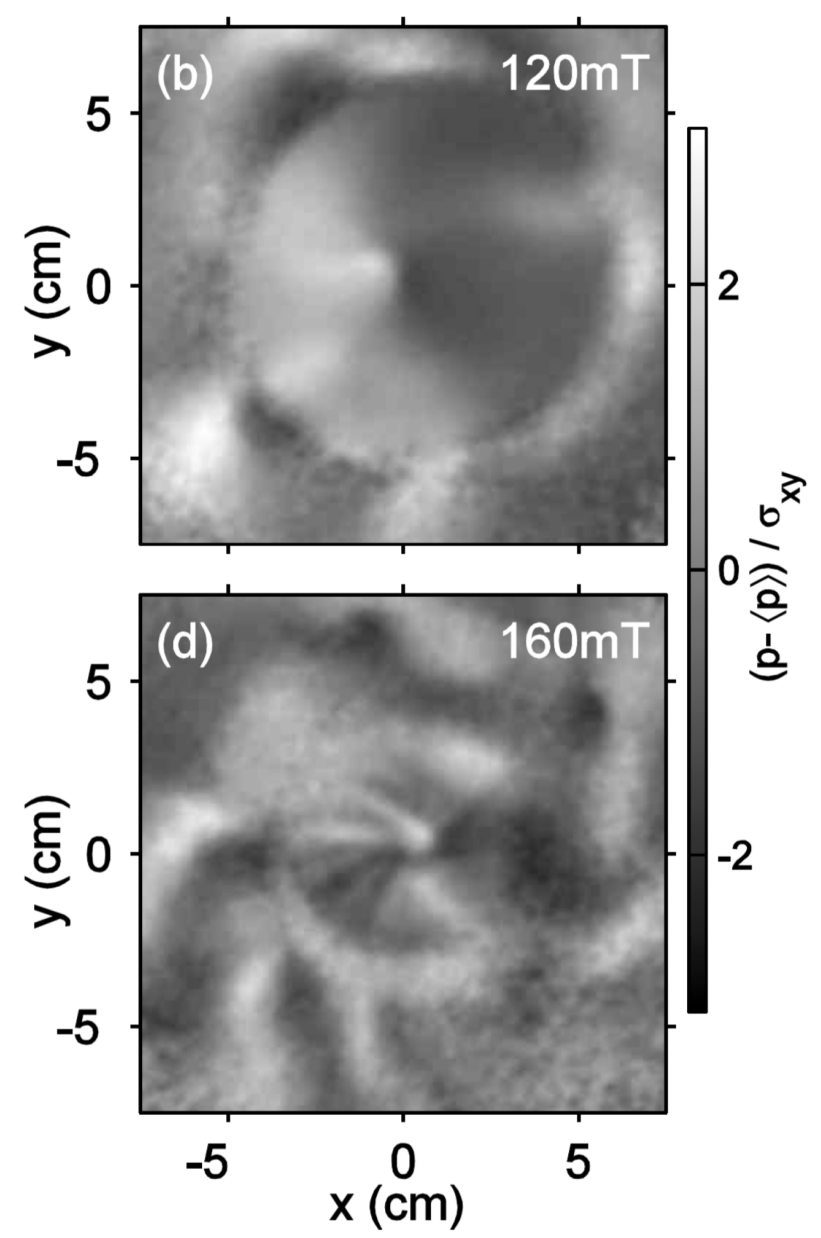

Figure 1.5: Images of the mode structure in CSDX [73]. A blue core is not observed in (b). Blue core mode shown in (d), with visible coherent structures out to a finite radius $r \approx 3 \mathrm{~cm}$, after which the plasma edge is turbulent. (C) 2015 IEEE.

occurs in opposing directions in 1) and 2), establishing a transport barrier in the region where the RDW instabilities dominate. This transport barrier sets the radial boundary of the blue core [73] and appears in Fig. 1.5 as the radial interface of regions of distinct modes. In CSDX, changing the boundary conditions between insulating and conducting materials $2.8 \mathrm{~m}$ from the antenna was sufficient to change plasma potential profiles, rotation profiles, and the Reynolds stress [74]. The axial boundary conditions modified radial currents and drastically changed fluctuation characteristics [74, 75]. In addition, spectral sidebands, markers of parametrically driven fluctuations, have been observed in electrostatic fluctuation spectra at different RF frequencies [72].

Measurements of ion temperature anisotropy in helicons suggest strong ion heating 


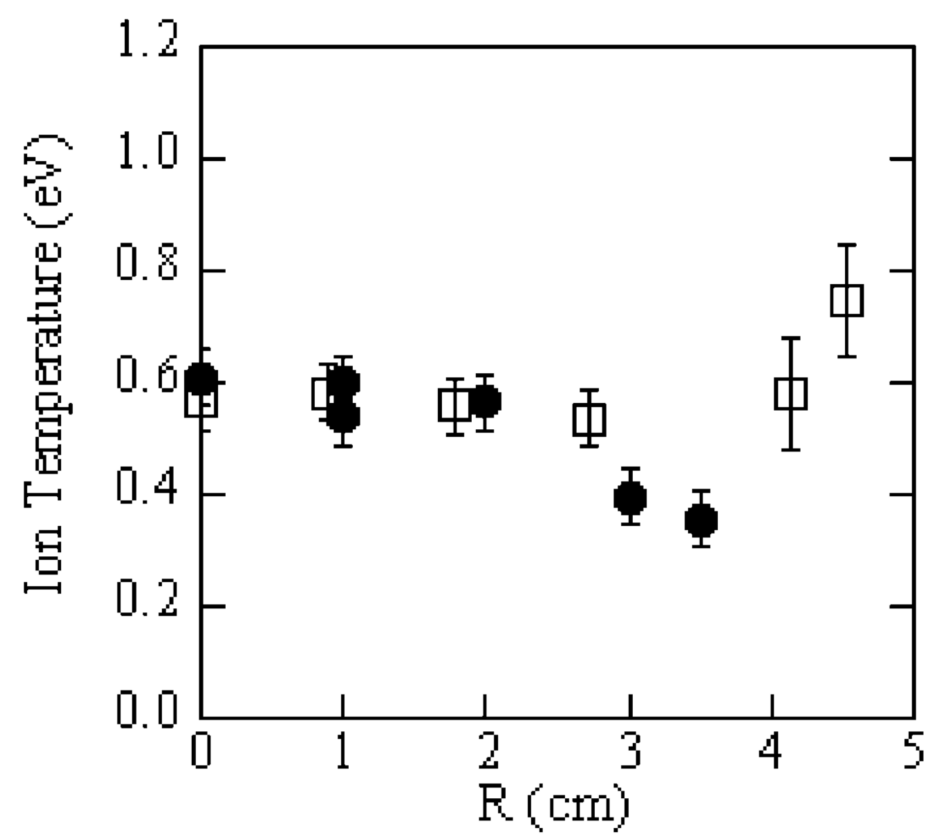

FIgURE 1.6: Radial $T_{i}$ profile showing anisotropic ion heating at outer radii [72]. Reprinted Fig. 5 with permission from J. L. Kline, E. E. Scime, R. F. Boivin, A. M. Keesee, and X. Sun, and V. S. Mikhailenko, Physical Review Letters, 88, 195002 (2002). Copyright (2002) by the American Physical Society.

in the edge in the direction perpendicular to the background magnetic field, as shown in Fig. 1.6 [72]. The helicon edge is characterized by low densities, which correspond to lower $\omega_{\mathrm{LH}}$ in the edge than in the center of the column. It is possible that the perpendicular slow wave modes arise in densities low enough to allow Landau damping on the ions. In this way, ion heating propagates radially from edge heating and is not primarily caused by wave-particle interactions with fast electrons [61]. The details of the underlying mechanisms involved remain the subject of active investigation.

\subsubsection{Density, temperature, and rotation profiles in HELIX}

Experiments using helicons for source plasmas must separate the effects of the gradients within the plasma column from gradients established by the boundary. Fig. 1.7 compares the laser-induced fluorescence (LIF) signal intensity profile from metastable argon ions to the profile of $n_{e}^{2} T_{e}^{1 / 2}$ [76]. The two profiles show remarkable agreement given the simplicity of the dependence. 


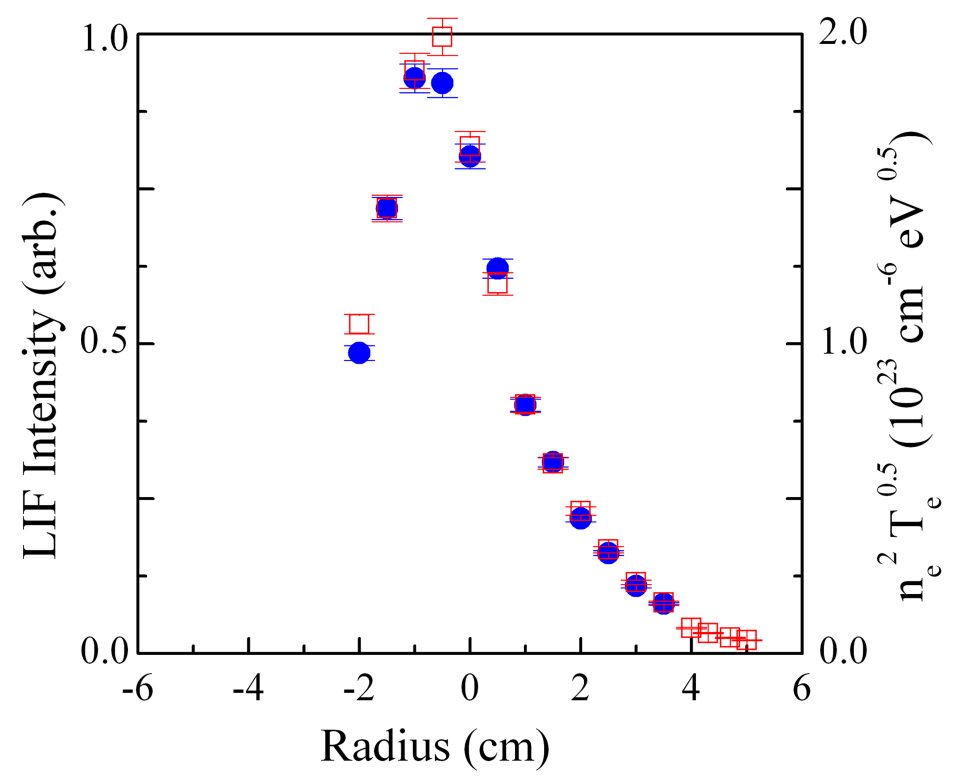

Figure 1.7: LIF intensity profile in HELIX, at $f_{\mathrm{RF}}=9.5 \mathrm{MHz}, P_{\mathrm{RF}}=750$ $\mathrm{W}, p=3.6 \mathrm{~m}$ Torr, and $B=716 \mathrm{G}[76]$. Blue circles show the LIF signal intensity. Red squares show $n_{e}^{2} T_{e}^{1 / 2}$. Reprinted with permission from $E$. E. Scime, personal communications.

Radial electric fields generate rotation of the plasma column, as shown in Fig. 1.8. In (a), the azimuthal ion flow changes sign near the mechanical axis of the machine and slows at larger radii. The ions exhibit negligible radial flows. The rotation rate varies significantly for different plasma conditions. Compare, for example, the plasma rotation in Fig. 1.8 to the measurements in Fig. 1.9, which show a $2 \mathrm{D}$ rotation map of perpendicular ion drifts at greater magnetic field magnitude and lower pressure. The ions in the core exhibit little drift, however they acquire speeds of several hundred $\mathrm{m} / \mathrm{s}$ within a few centimeters. The rotation speed and profile are observed to depend on the magnetic field magnitude [77]. $T_{i}$ is shown in Fig. 1.9 (b) to be relatively uniform over the innermost part of the plasma, where $r \lesssim 3 \mathrm{~cm}$.

Axial ion flows in the CSDX cylindrical helicon source were observed to be several hundred $\mathrm{m} / \mathrm{s}$ at fill pressure $p=3.2 \mathrm{mTorr}$ and $\mathrm{RF}$ power $P_{\mathrm{RF}}=1800 \mathrm{~W}$, as shown in Fig. 1.10 [78]. At low magnetic field magnitudes, the axial flow profile is relatively flat. At higher fields, the flow decreases and perhaps changes sign at the outermost radii. 

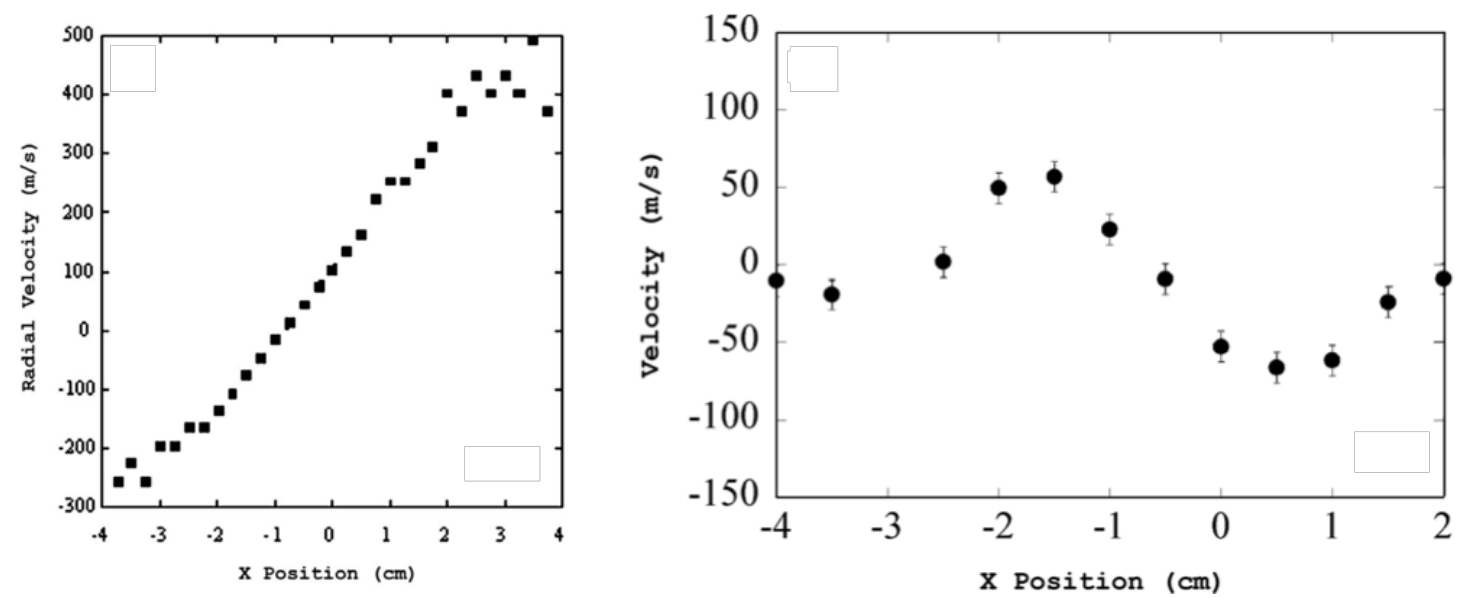

Figure 1.8: (a) Radial Ar-II velocity measured with LIF at $f_{\mathrm{RF}}=9.5 \mathrm{MHz}$, $P_{\mathrm{RF}}=750 \mathrm{~W}, p=3.8 \mathrm{mTorr}$, and $B=652 \mathrm{G}$; and (b) radial He-I neutral flows in HELIX, at $f_{\mathrm{RF}}=9.5 \mathrm{MHz}, P_{\mathrm{RF}}=750 \mathrm{~W}, p=34 \mathrm{mTorr}$, and $B=500$ $\mathrm{G}$ [76]. The axis of symmetry is located at $X \approx-0.5 \mathrm{~cm}$. Image at left reprinted from E. Scime, R. Hardin, C. Biloiu, A. M. Keesee, and X. Sun, Physics of Plasmas 14, 043505 (2007), with the permission of AIP Publishing. Image at right from E. Scime, personal communications.

\subsubsection{Neutral depletion in the plasma core}

Momentum exchange collisions between ions and neutral particles are an important component of the physics of plasma boundaries, especially when helicon sources are used to generate the test plasma. In contrast to primarily azimuthal and axial ion flows, inward neutral flows across the plasma density gradient are observed in LIF flow measurements, as shown in part (b) of Fig. 1.8. These inward flows suggest inelastic collisions such as charge exchange or ionization play a role in establishing the helicon equilibrium [79]. Collision rates depend on the density of the target species. While local ion densities can be measured by Langmuir probes, it is difficult to measure absolute neutral densities in argon for estimating collision rates, and measurable quantities are combined with collisional-radiative (CR) models to estimate neutral density profiles. An example profile, calculated using a CR model for a plasma in $p=6.0$ mTorr with $B=750 \mathrm{G}$ and $P_{\mathrm{RF}}=300$ W, is shown in Fig. 1.11 along with the estimated momentum-transfer collision rate profile $[76,80]$. These profiles indicate high ionization or charge exchange collisions, with neutral depletion of $30-40 \%$ in the core at these plasma parameters. Neutral pressures as low as one-tenth of the pre-discharge fill pressure have been 

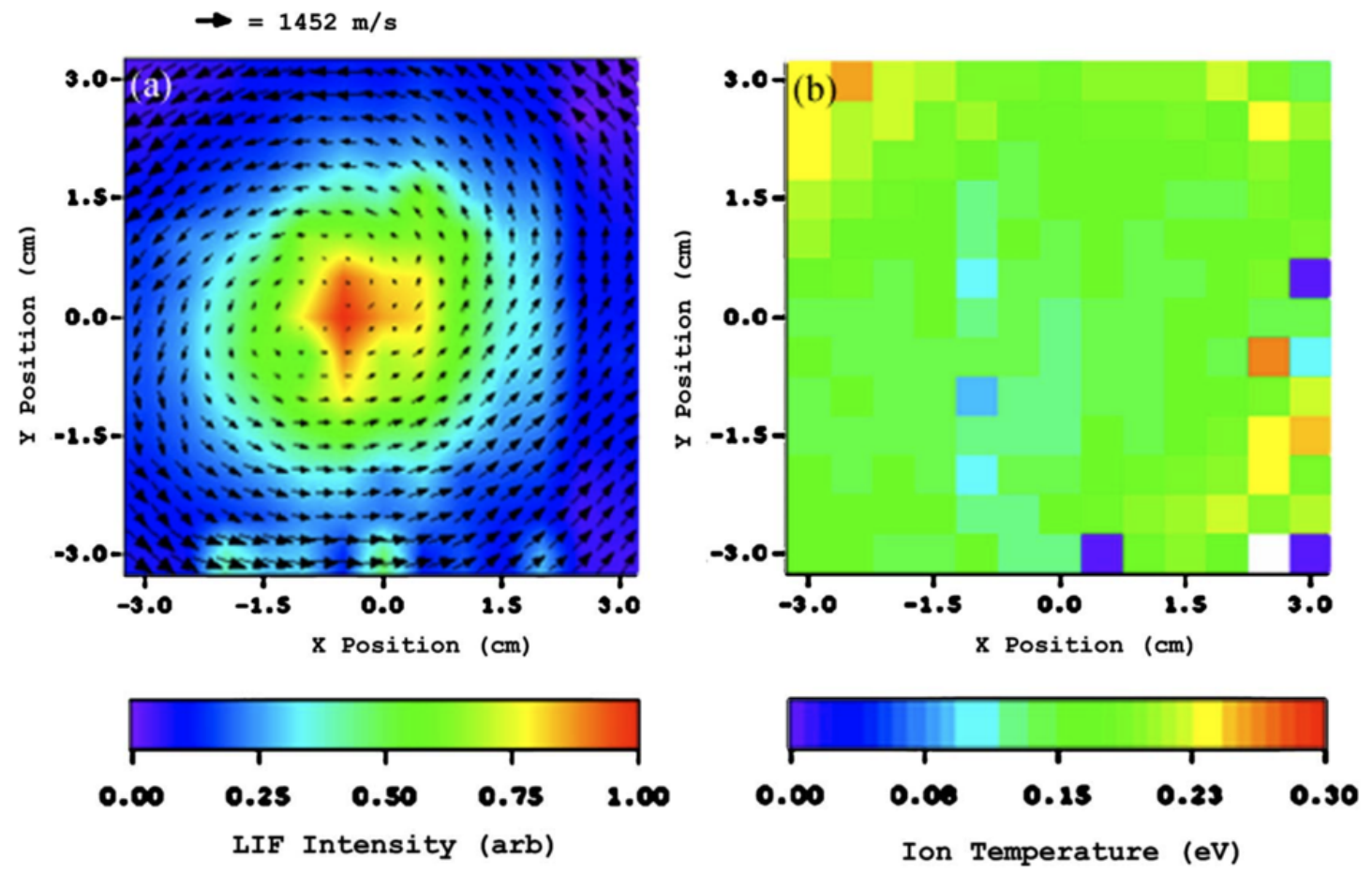

Figure 1.9: Heatmap of plasma column rotation in HELIX, measured with LIF, at $f_{\mathrm{RF}}=9.5 \mathrm{MHz}, P_{\mathrm{RF}}=750 \mathrm{~W}, p=1.8 \mathrm{mTorr}$, and $B=730 \mathrm{G}$ [76]. Arrows indicate ion flow speed. Color indicates (a) LIF intensity in and (b) $T_{i}$. Reprinted from E. Scime, R. Hardin, C. Biloiu, A. M. Keesee, and X. Sun, Physics of Plasmas 14, 043505 (2007), with the permission of AIP Publishing.

observed for similar argon plasma conditions [79, 81-83]. As a result, models assuming flat profiles and using neutral densities from pressure gauges located in the chamber wall risk misrepresenting the collision rates at the center of the column.

Absolute densities have been measured in helicon plasmas in krypton in the CHEWIE device [84]. As expected, they show a linear increase in neutral density with fill pressure, and $n_{n} \approx 10^{20} \mathrm{~m}^{-3}$ at $p=3.6 \mathrm{~m}$ Torr. At $P_{\mathrm{RF}}=600 \mathrm{~W}$, the neutral density is insensitive to magnetic field magnitude until $B \geq 600 \mathrm{G}$, above which the neutral density decreases as the plasma density rises. Plasma and neutral density profiles, and dependence of the Kr-I and Kr-II densities on magnetic field and fill pressure, are shown in Fig. 1.12. At $B=600 \mathrm{G}$ and $P_{\mathrm{RF}}=600 \mathrm{~W}$, similar conditions to the measurements reported here, the Kr-II profile is centrally peaked, but the Kr-I profile is hollow, showing perhaps a 50\% neutral depletion within $|r| \leq 1 \mathrm{~cm}$ (see (b), Fig. 1.12). 


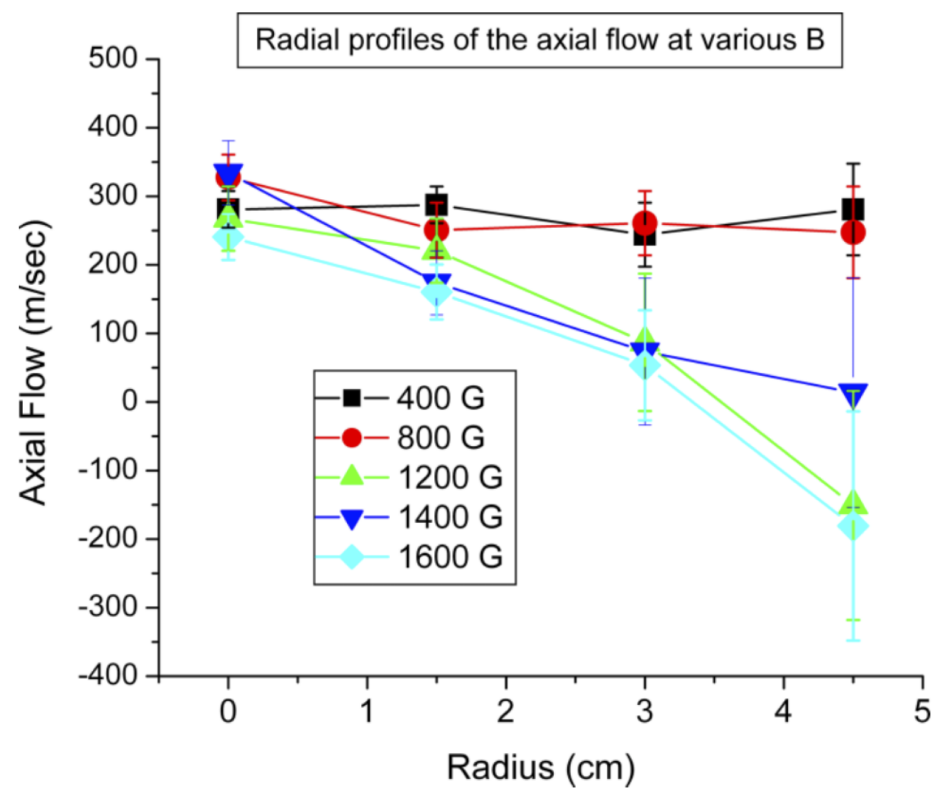

FiguRE 1.10: Axial flow dependence on magnetic field in CSDX measured with LIF with $f_{\mathrm{RF}}=13.65 \mathrm{MHz}, P_{\mathrm{RF}}=1800 \mathrm{~W}$, and $p=3.2$ mTorr [78]. Reprinted from S. Chakraborty Thakur, K. Adriany, J. J. Gosselin, J. McKee, E. E. Scime, S. H. Sears, and G. R. Tynan, Physics of Plasmas 87, $11 E 513$ (2016), with the permission of AIP Publishing.
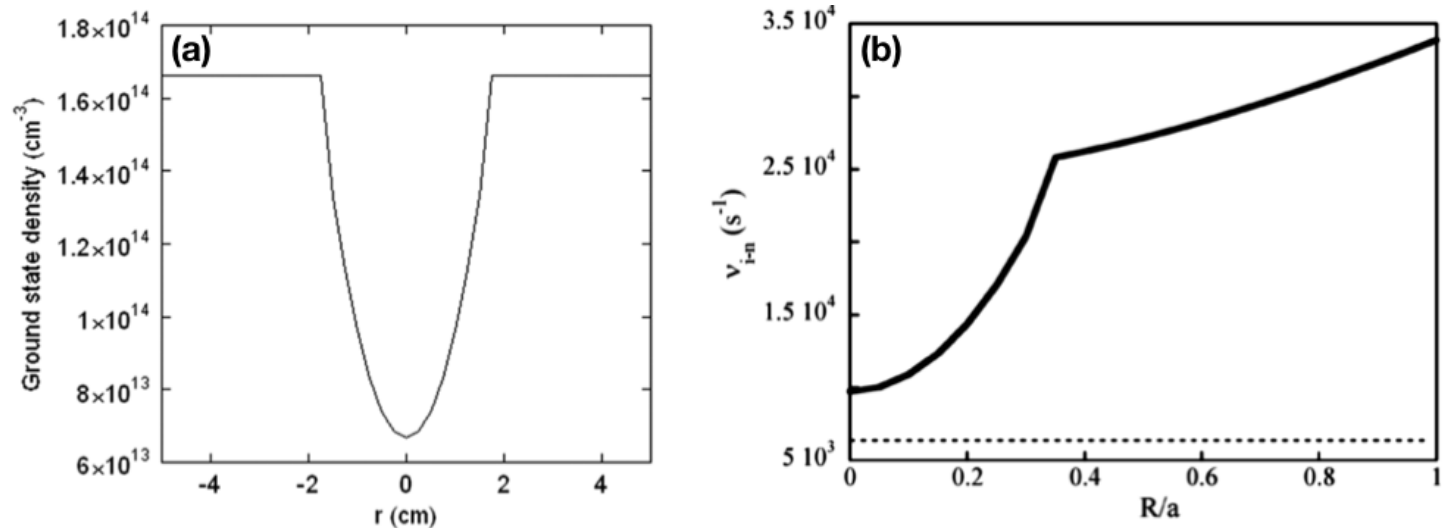

Figure 1.11: (a) Ar-I density and (b) ion-neutral collision rate profiles for $B$ $=750 \mathrm{G}, P_{\mathrm{RF}}=300 \mathrm{~W}, p=6.0 \mathrm{mTorr}[76,80]$. Image at right reprinted from E. Scime, R. Hardin, C. Biloiu, A. M. Keesee, and X. Sun, Physics of Plasmas 14, 043505 (2007), with the permission of AIP Publishing.

\subsection{Dissertation overview}

This dissertation describes the measurement of ion, neutral, and electron dynamics in a boundary region with an oblique magnetic field. Where possible, measurements of all three spatial and three velocity components are acquired. The details of the boundary that was created to study the magnetic presheath are described in 

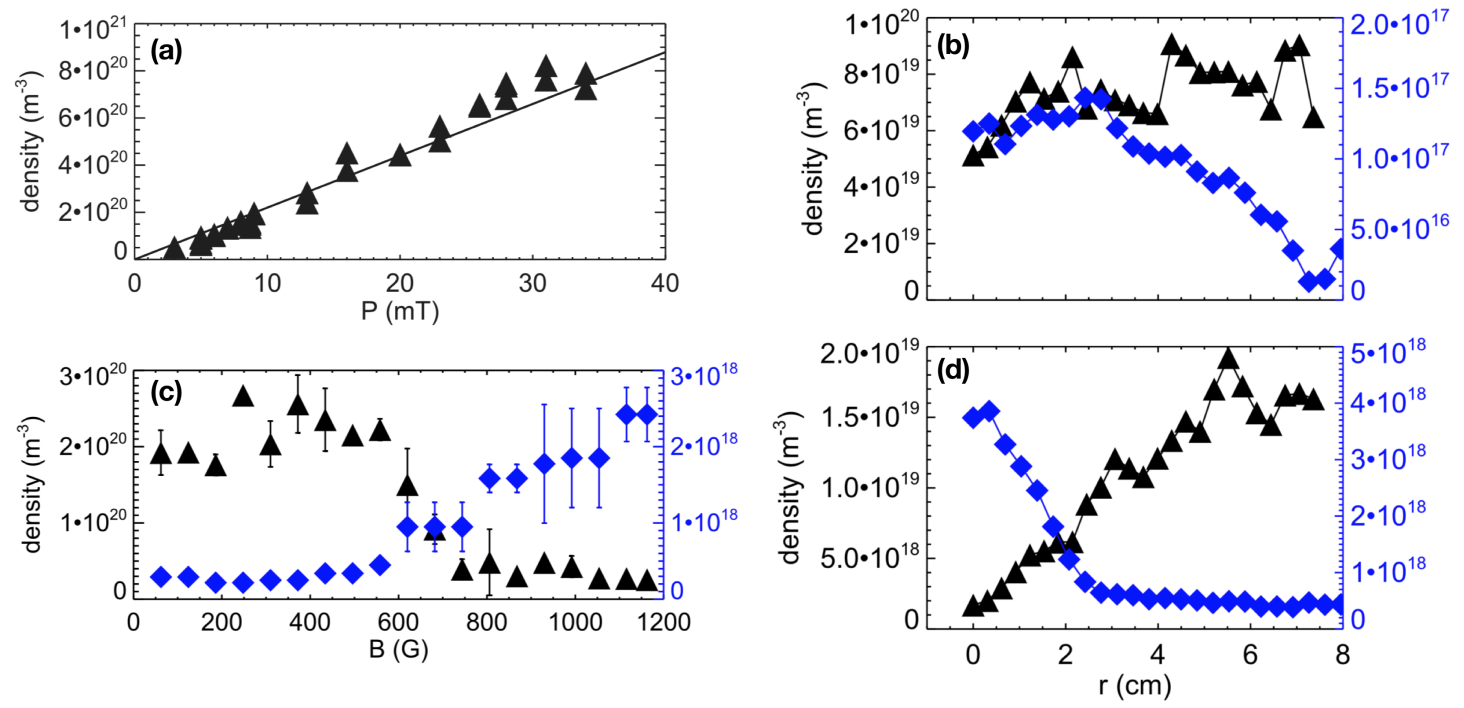

Figure 1.12: Kr-I density profiles and dependences in CHEWIE [84]. (a) Pressure dependence of two-photon absorption LIF (TALIF) signal. (b) Kr-I density (black triangles, left axis) and Kr-II density (blue diamonds, right axis) profile at $f_{\mathrm{RF}}=13 \mathrm{MHz}, P_{\mathrm{RF}}=600 \mathrm{~W}$, and $B=600 \mathrm{G}$. (c) Magnetic field dependence of Kr-I density (black triangles, left axis) and Kr-II density (blue diamonds, right axis) at $P_{\mathrm{RF}}=600 \mathrm{~W}$. (d) Kr-I density (black triangles, left axis) and Kr-II density (blue diamonds, right axis) profile at $f_{\mathrm{RF}}=11 \mathrm{MHz}$, $P_{\mathrm{RF}}=800 \mathrm{~W}$, and $B=1200 \mathrm{G}$. Reprinted from R. M. Magee, M. E. Galante, N. Gulbrandsen, D. W. McCarren, and E. E. Scime, Physics of Plasmas 19, 123506 (2012), with the permission of AIP Publishing.

Chapter 2. Described in this chapter are the coordinate systems used to map the boundary region. The HELIX facility which provides the consistent test plasma is described as well, along with a brief description of several key components.

Chapter 3 describes the diagnostics used for the measurements presented. The 3 -axis probe is introduced, and its construction and calibration methods are detailed. The plasma conditions in the boundary were not known a priori, so a litany of Langmuir probe analysis methods are described for extracting plasma quantities from this diagnostic. Several important pitfalls are highlighted. Chapter 3 describes the laser-induced fluorescence technique that was used to measure the ion and neutral velocity distributions, including transition schemes, calibration, and a list of possible artifacts. The results from the experimental campaign are presented in Chapter 4, where they are compared to collisional fluid and Particlein-Cell simulations and accompanied by some implications for magnetic presheath 
theory. Chapter 5 summarizes the results of the project and provides some additional discussion. 


\section{Chapter 2}

\section{Experimental Apparatus}

\subsection{The HELIX/LEIA facility}

The experiments reported here were conducted in the Hot hELIcon eXperiment (HELIX) attached to the Large Experiment on Instabilities and Anisotropies (LEIA) facility at West Virginia University. A schematic of the HELIX chamber is shown in Fig. 2.1 and typical ranges for engineering quantities are listed in Tab. 2.1. A recent review of the facility is available in Ref. [59], which contributes to much of this discussion.

The HELIX chamber consists of a $63 \mathrm{~cm} \mathrm{x} 10 \mathrm{~cm}$ dia. cylindrical Pyrex tube situated between a $91 \mathrm{~cm}$ x $15 \mathrm{~cm}$ dia. stainless steel tube and a Pyrex cross. The end of the stainless steel section opposite the Pyrex attaches coaxially to LEIA, a $4 \mathrm{~m}$ long, $2 \mathrm{~m}$ diameter aluminum expansion chamber. A uniform magnetic field

\begin{tabular}{lccc}
\hline \hline Parameter & Typical & Minimum & Maximum \\
\hline$f_{\mathrm{RF}}[\mathrm{MHz}]$ & 9.5 & 0.3 & 30 \\
$P_{\mathrm{RF}}[\mathrm{W}]$ & 650 & 0 & 2000 \\
$B[\mathrm{~T}]$ & 0.06 & 0 & 0.12 \\
Mass flow $[\mathrm{sccm}]$ & 20 & 0.1 & 200 \\
Pump rate $[\mathrm{L} / \mathrm{s}]$ & 3200 & 0 & 3750 \\
\hline
\end{tabular}

TABLE 2.1: HELIX facility engineering parameters [59]. 


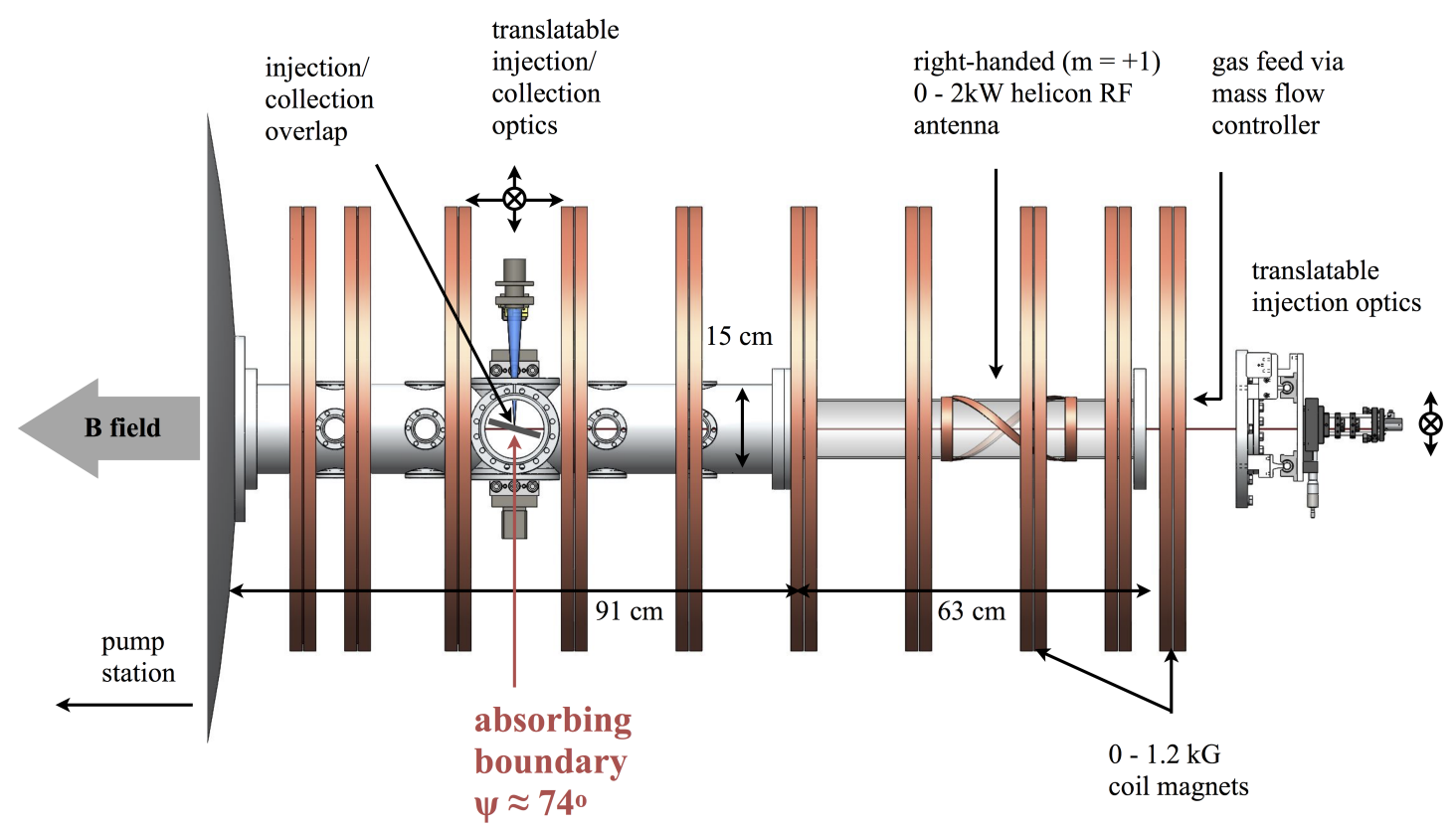

Figure 2.1: Overview schematic of the HELIX chamber.

up to $0.12 \mathrm{~T}(1225 \mathrm{G})$, with field variation $\delta B / B \lesssim 1 \%$, is established in HELIX by ten water-cooled copper channel pancake electromagnets driven by a parallel pair of Xantrex XFR DC power supplies.

Optical access to the HELIX plasma is provided by sixteen 2.75" Conflat ports, spaced 6" apart at top, bottom, and both sides of the HELIX chamber. At the ports nearest the antenna, static telescopes with fiber ports maintain a fixed, optimized focus on the center of the plasma. This optical arrangement is used for standard measurements and troubleshooting. Halfway down the length of the HELIX chamber, a 6" Conflat cross-port provides large volume optical and probe access to the plasma. This is the location of most of the experiments reported in this document.

Vacuum in the chamber is maintained by a $550 \mathrm{~L} / \mathrm{s}$ turbomolecular pump attached to the bottom of the Pyrex cross and two $1600 \mathrm{~L} / \mathrm{s}$ turbomolecular drag pumps on the rear wall of LEIA. Each of these pumps is backed by an oil-free diaphragm pump. The large chamber volume is reduced from atmospheric pressure to 0.5 Torr by an oil-free roots pump. This vacuum system ensures that the surfaces inside the chamber are never exposed to pump oil. The chamber readily obtains vacuum 


\begin{tabular}{lccc}
\hline \hline Parameter & Typical & Minimum & Maximum \\
\hline$n\left[\mathrm{~m}^{-3}\right]$ & $5 \times 10^{18}$ & $\sim 5 \times 10^{15}$ & $\sim 3 \times 10^{19}$ \\
$T_{\text {Ar-I }}[\mathrm{eV}]$ & 0.027 & 0.025 & - \\
$T_{\text {Ar-II }}[\mathrm{eV}]$ & 0.4 & 0.15 & 2.5 \\
$T_{e}[\mathrm{eV}]$ & 4 & 1.5 & 15 \\
$p_{\text {vac }}[$ Torr $]$ & $1 \times 10^{-7}$ & $7 \times 10^{-8}$ & $5 \times 10^{-7}$ \\
$p_{\text {gas }}[$ mTorr $]$ & 4 & 0.1 & 100 \\
\hline \hline
\end{tabular}

TABLE 2.2: HELIX facility plasma quantities [59].

pressures below $5 \times 10^{-7}$ Torr, a pressure that improves to $7-8 \times 10^{-8}$ Torr after several hours of steady plasma operation.

Gas is fed into the chamber from the top of the Pyrex cross. Plasmas in HELIX are formed from argon, helium, nitrogen, hydrogen, xenon, and krypton gases. Gas flow is controlled with calibrated MKS 1179 mass flow valves and a MKS PR-4000 mass flow controller. The mass flow operates from a minimum of $0.1 \mathrm{sccm}$ up to $200 \mathrm{sccm}$. The pressure inside HELIX is manipulated by the mass flow controller, the location of gas injection, and by opening or closing the gate valves to the vacuum pumps. Pre-plasma fill pressures range from 0.1 to $100 \mathrm{~m}$ Torr.

Plasmas in HELIX are created by a $19 \mathrm{~cm}$ long, half wave, $m=+1$ helical antenna, which is wrapped around the cylindrical Pyrex section of the chamber. The antenna is driven at RF frequencies between 8.5 and $16 \mathrm{MHz}$. Up to $1.5 \mathrm{~kW}$ of forward $\mathrm{RF}$ power is produced by a single ENI A1000 RF power supply, driven by a Wavetek 80 function generator. Typical plasma conditions are shown in Tab. 2.2.

\subsection{1 $\pi$ network matching circuit}

RF power is coupled to the plasma by driving the antenna through a balanced $\pi$ network matching circuit. A diagram of the matching circuit on HELIX is shown in Fig. 2.2. In a $\pi$ network matching circuit, neither side of the antenna is electrically connected to ground. One side of the antenna is separated from the ground 


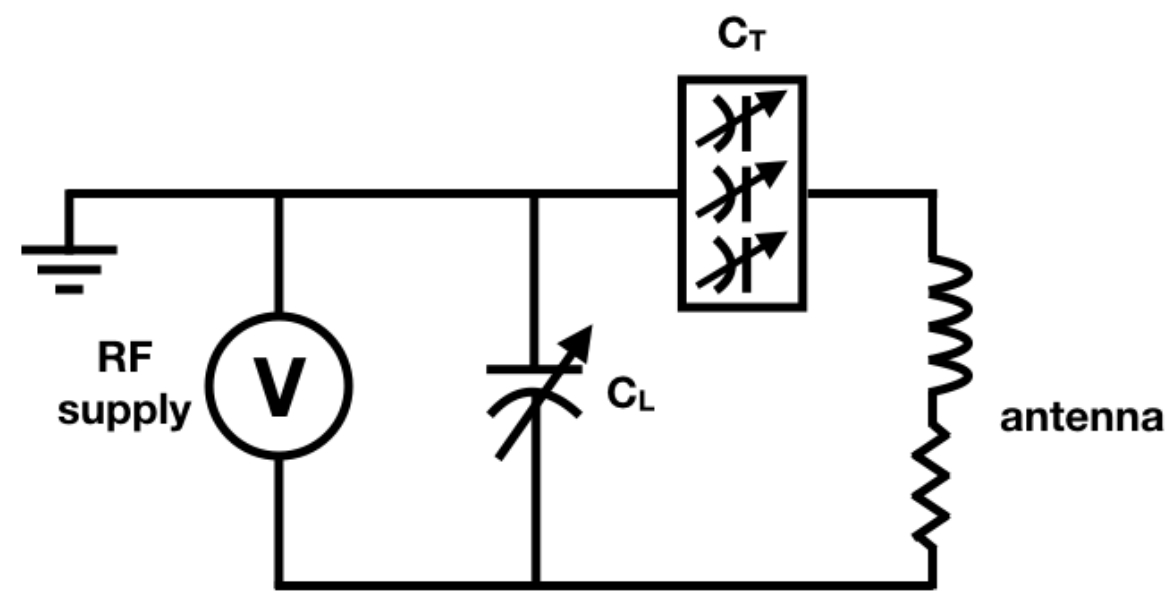

Figure 2.2: Circuit diagram: HELIX $\pi$ matching circuit [59]. The load capacitor is placed in parallel with the antenna. Three tuning capacitors are placed in series with the antenna. E. E. Scime, P. A. Keiter, M. M. Balkey, J. L. Kline, X. Sun, A. M. Keesee, R. A. Hardin, I. A. Biloiu, S. Houshmandyar, S. Chakraborty Thakur, J. Carr, M. Galante, D. McCarren, and S. Sears, The hot hELicon eXperiment (HELIX) and the large experiment on instabilities and anisotropy (LEIA), Journal of Plasma Physics, 81, 1, 345810103, reproduced with permission.

reference by three tuning capacitors in parallel with each other. Two of these capacitors are Jennings UCSL 250 tunable capacitors with a 4-250 pF range. The other capacitor is a Jennings UCSL 500 tunable capacitor with a 5-500 pF range. These tuning capacitors allow RF power to be driven over a theoretical range of $0.3-30 \mathrm{MHz}$, however the RF frequency for all measurements reported here is 9.5 MHz. The side of the antenna away from the tuning capacitor set is connected to the negative pole of the power supply. The load capacitor, a Jennings CVCD 2000 tunable capacitor with a 20-2000 pF range and 40 A maximum current, is connected parallel to the antenna and the power supply on the side of the tuning capacitors away from the antenna. The capacitors connect to the antenna and the power supply via a $6.2 \mathrm{~mm}$ thick silver-plated copper bar, which serves as a strong electrical connection, mechanical support, and heat sink [59].

The goal of a $\pi$ network matching circuit is to create a $(50+0 i) \Omega$ impedance that matches the impedance of the transmission lines, minimizing the reflected power. (The following discussion adapts [85].) The impedance should be entirely real. The impedance of the circuit, with the tuning capacitors combined into a 
single quantity $C_{\mathrm{T}}$ and the load capacitance $C_{\mathrm{L}}$, is

$$
\left.Z=\left(Z_{\mathrm{L}}^{-1}+Z_{\mathrm{T}}^{-1}\right)\right)^{-1}
$$

where $Z_{\mathrm{L}}=1 / i \omega C_{\mathrm{L}}, Z_{\mathrm{T}}=R+i X+1 / i \omega C_{\mathrm{T}}$, and $\omega$ is the RF driving frequency. $X$ is the reactance of the antenna and changes depending on whether the load is primarily inductive or capacitive. Inserting $Z_{\mathrm{L}}$ and $Z_{\mathrm{T}}$ into Eq. (2.1), and defining $Q=X-1 / \omega C_{\mathrm{T}}$ gives

$$
\begin{aligned}
Z & =\left[i \omega C_{\mathrm{L}}+\frac{1}{R+i X+1 / i \omega C_{\mathrm{T}}}\right]^{-1}=\frac{R+i\left(X-1 / \omega C_{\mathrm{T}}\right)}{1+i \omega C_{\mathrm{L}}\left[R+i\left(X-1 / \omega C_{\mathrm{T}}\right)\right]} \\
& =\frac{R+i Q}{1-\omega C_{\mathrm{L}} Q+i \omega R C_{\mathrm{L}}} .
\end{aligned}
$$

Making the denominator purely real the impedance becomes

$$
\begin{aligned}
Z & =\frac{(R+i Q)\left(1-\omega C_{\mathrm{L}} Q-i \omega R C_{\mathrm{L}}\right)}{\left(1-\omega C_{\mathrm{L}} Q\right)^{2}+\left(\omega R C_{\mathrm{L}}\right)^{2}}=\frac{R-i\left[\omega C_{\mathrm{L}}\left(R^{2}+Q^{2}\right)-Q\right]}{\left(1-\omega C_{\mathrm{L}} Q\right)^{2}+\left(\omega R C_{\mathrm{L}}\right)^{2}} \\
& ==\frac{R-i\left[\omega C_{\mathrm{L}}\left(R^{2}+Q^{2}\right)-Q\right]}{1-2 \omega C_{\mathrm{L}} Q+\left(\omega C_{\mathrm{L}}\right)^{2}\left(R^{2}+Q^{2}\right)}
\end{aligned}
$$

$\operatorname{Im}(Z)=0$ implies $\omega C_{\mathrm{L}}\left(R^{2}+Q^{2}\right)-Q=0$, or equivalently

$$
\left(\omega C_{\mathrm{L}}\right)^{2}\left(R^{2}+Q^{2}\right)=\omega C_{\mathrm{L}} Q
$$

Eq. (2.3) can now be rewritten to match $\operatorname{Re}(Z)=R_{\mathrm{o}}$ :

$$
\omega C_{\mathrm{L}} Q=1-R / R_{\mathrm{o}}
$$

If $A \equiv \omega C_{\mathrm{L}} Q$, solving the quadratic in Eq. (2.4) gives

$$
\begin{aligned}
2 A-1 & = \pm\left(1-4 \omega^{2} C_{\mathrm{L}}^{2} R^{2}\right)^{1 / 2} \\
\left(1-2 R / R_{\mathrm{o}}\right)^{2} & =1-4 \omega^{2} C_{\mathrm{L}}^{2} R^{2} .
\end{aligned}
$$



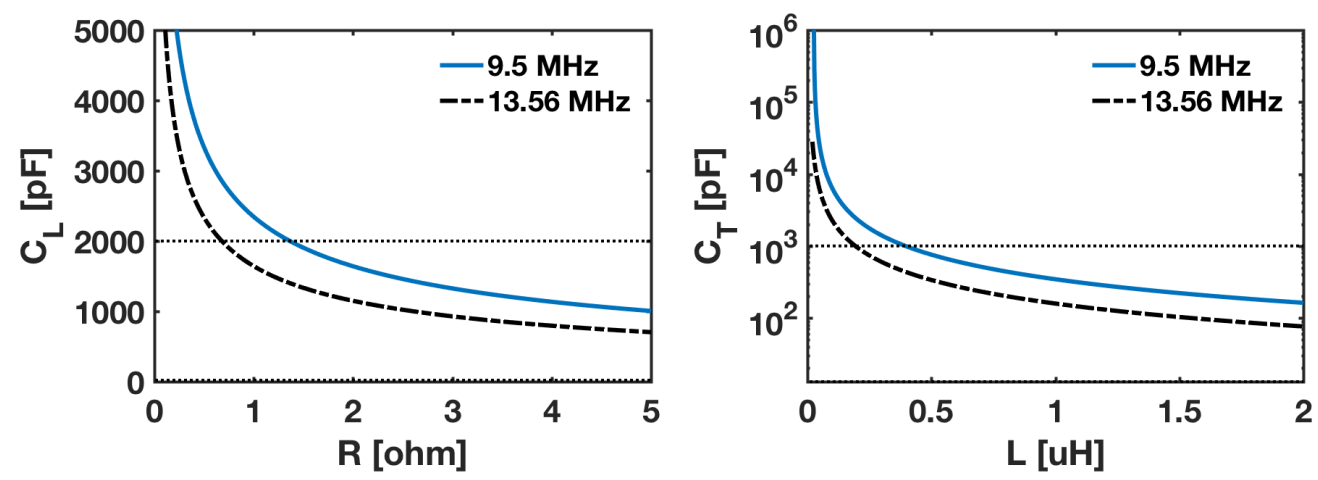

FiguRE 2.3: HELIX $\pi$ matching circuit load and tuning capacitance for a range of antenna resistance and inductance. The 20 - 2000 pF HELIX load capacitance is appropriate for antenna resistances above approximately $1.4 \Omega$. The tuning capacitance is weakly dependent on the antenna resistance, and $C_{\mathrm{T}}<1000 \mathrm{pF}$ is appropriate above approximately $0.4 \mu \mathrm{H}$.

Therefore

$$
C_{\mathrm{L}}=\frac{1}{2 \omega R}\left[1-\left(1-\frac{2 R}{R_{\mathrm{o}}}\right)^{2}\right]^{1 / 2}
$$

and from Eq. (2.5)

$$
C_{\mathrm{T}}=\left[\omega X-\frac{1-R / R_{\mathrm{o}}}{C_{\mathrm{L}}}\right]^{-1}
$$

For a helicon discharge, $X$ is expected to be primarily inductive, so that $X=$ $X_{\mathrm{L}}=\omega L$. In this case, Eq. (2.7) becomes $C_{\mathrm{T}}^{-1}=\omega^{2} L-\left(1-R / R_{\mathrm{o}}\right) / C_{\mathrm{L}}$. At large $L, C_{\mathrm{T}} \propto L^{-1}$, the matching does not depend strongly on the inductance of the antenna, and the circuit establishes a stable discharge. In practice, the capacitors are tuned to achieve this condition. Fig. 2.3 shows the predicted range required for the HELIX load capacitor. The 20 - 2000 pF HELIX load capacitance is appropriate for antenna resistances above approximately $1.4 \Omega$, and $C_{\mathrm{T}}<1000$ $\mathrm{pF}$ is appropriate above approximately $0.4 \mathrm{\mu H}$. In essence, for a given antenna, the capacitor set determines the range of frequencies over which a stable discharge can be sustained. In the discharges used in the experiments reported in this document, which are created in 3.6 mTorr of argon, a magnetic field of 600 gauss, and an RF frequency of $9.5 \mathrm{MHz}$, the HELIX matching circuit is capable of reducing the reflected $\mathrm{RF}$ power below $<3 \mathrm{~W}$ out of $650 \mathrm{~W}$. 


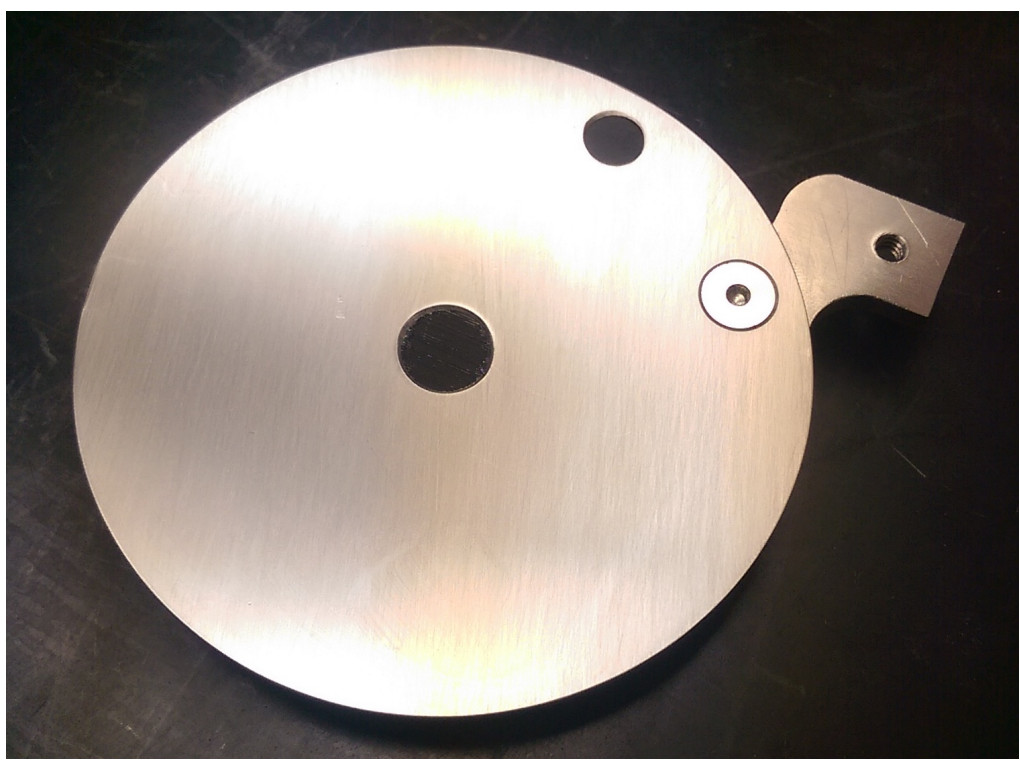

Figure 2.4: Photograph of the (Type 316) stainless steel absorbing boundary.

\subsection{Absorbing boundary}

The test boundary in these experiments, shown in Fig. 2.4, is created from a 1 mm thick, $\varnothing 7.62 \mathrm{~cm}$ Type 316 stainless steel disk. The boundary dimensions are intended to be large enough that the behavior observed at the center will not be influenced by edge effects, since the distance between the sample region to the edge is larger than the sheath and presheath scales, the charge exchange length, and the ion and electron gyro-radii. The disk is fixed to a U-shaped Type 316 bracket that supports the disk and prevents it from sagging or deflecting. The bracket also provides a strong electrical connection between the disk and the post that grounds the disk to the chamber wall through a low-impedance path. The impedance between the outer edge of the disk and the nearby flange is measured with a digital multimeter to be less than the tip-to-tip impedance of the probes, i.e., $<0.3 \Omega$. Rotating the post outside the chamber changes the angle between the boundary within the optical access at the center of the cross port.

At the center of the disk, a razor blade beam dump sits within a $\varnothing 9.5 \mathrm{~mm}$ hole so that the edges of the blades are flush with the disk surface. The beam dump is used to attenuate false "signals" from laser reflection off the boundary surface during laser-induced fluorescence (see Sec. 3.3). Each blade is 0.009" thick, and the 


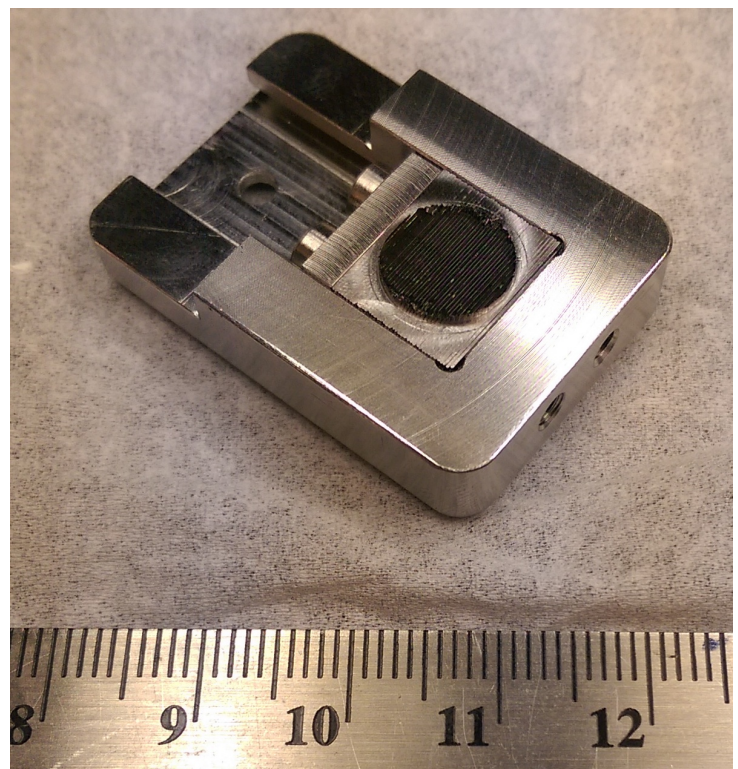

Figure 2.5: Photograph detail of the (Type 316 SS) laser beam dump. Dark, circular region is the effective absorption area of the beam dump.

tight grooves of the razor blade stack absorb the incident beam, providing a way to differentiate reflected signal from non-Maxwellian spectral features. Commercially available razor blades are fabricated from magnetic, high-carbon 440C stainless steel. Beam dumps fabricated from these blades distort the local magnetic field lines. To ensure that the beam dump does not affect the local field structure and to maintain the angle of incidence of the field lines on the boundary surface, each blade of the beam dump is fabricated from non-magnetic, austenitic Type 316 stainless steel (no special letter designation). A photograph of the beam dump in its housing is shown in Fig. 2.5. The stacked edges of the blades are cut to form a dark, relieved circle that fits into the hole at the boundary center. The blades are shown in their Type 316 housing, which attaches to the underside of the Ushaped mount bracket. A photograph of the entire boundary assembly immersed in a plasma is shown in Fig. 2.6.

The boundary perturbation to the magnetic field was measured with a gaussmeter (see Ch. 3 for details). The results, shown in Fig. 2.7 for the on-axis field, confirm that the perturbation from the presence of the plate is everywhere less than $1 \%$. 


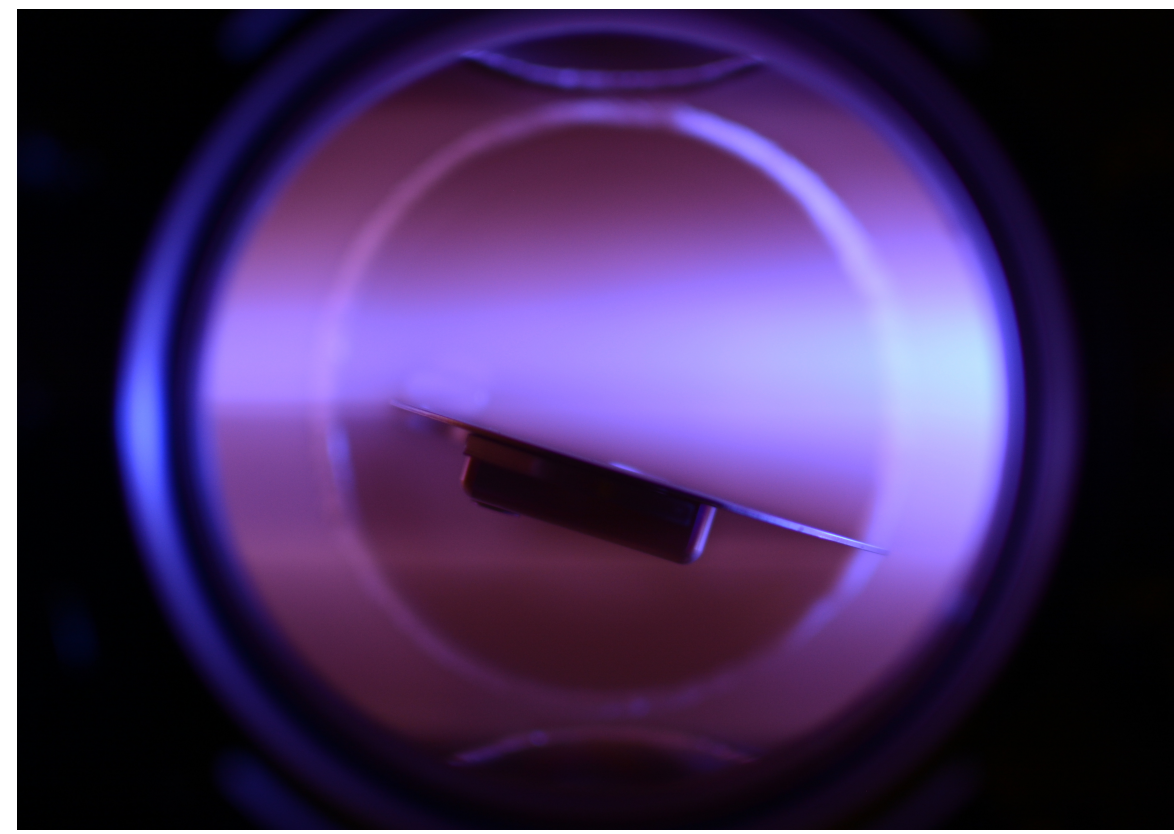

FiguRE 2.6: Photograph of the absorbing boundary immersed in the HELIX plasma.

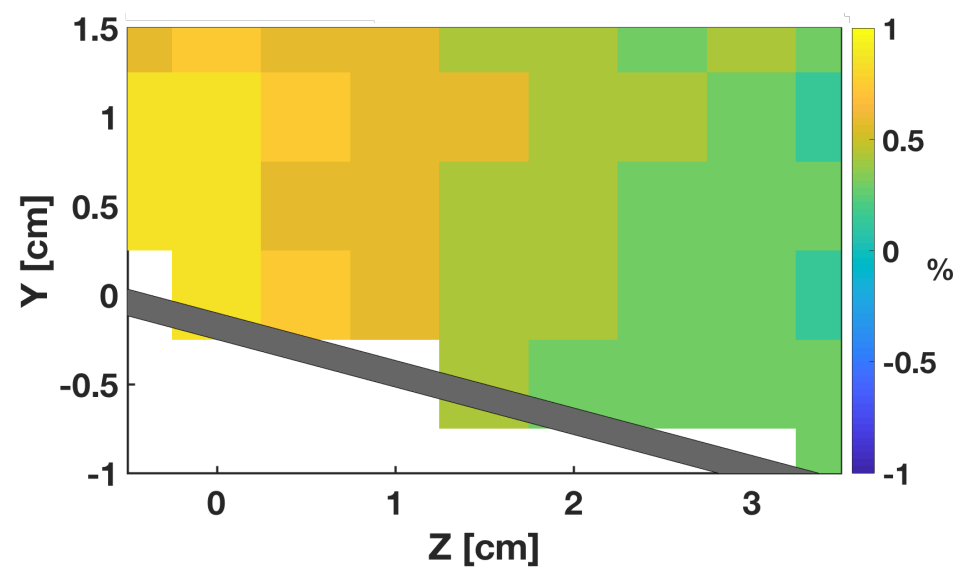

Figure 2.7: Percentage perturbation from the presence of the absorbing boundary. The color axis represents $\left(B_{\text {out }}-B_{\text {in }}\right) / B_{\text {out }}[\%]$.

\subsection{Boundary coordinates}

Two coordinate systems are used to describe spatial variation in the boundary environment for the experiments in this work. The first coordinate system is dictated by the LIF translation stages. The second system is determined by physics models. 


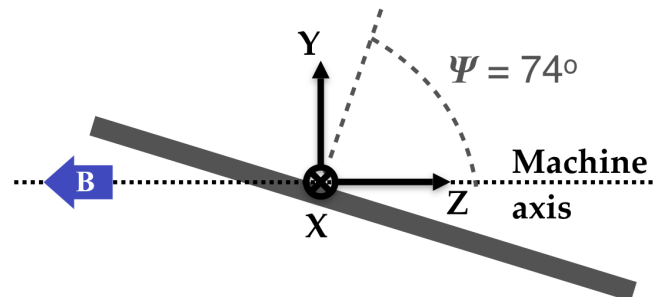

Translational stage coordinates

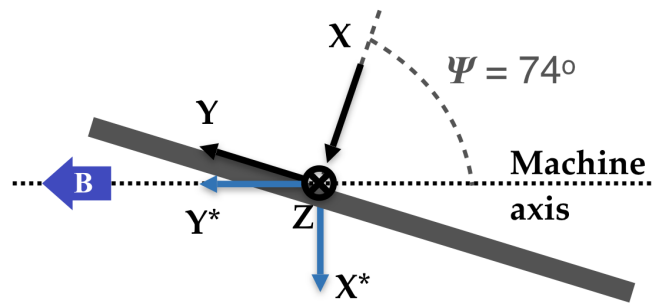

Simulation coordinates

FiguRE 2.8: Translational stage and physics coordinate systems

\subsubsection{Translation stage coordinates}

The experimental results will be presented in the coordinate system employed by simulations. Initially, however, the translation stage positions and all notes were recorded in the lab or "translational stage" coordinate system, which is described here. The origin of this coordinate system on HELIX is located along the mechanical chamber axis, $69 \mathrm{~cm}$ from the end of the antenna, where the chamber axis intersects the boundary surface (see Fig. 2.1). The $x$ axis is perpendicular to the gravity vector, i.e., parallel to the floor, with $x>0$ toward the lab doors, to the right of the chamber axis passing from the back of the glass cross to the back of LEIA. The $y$ axis is antiparallel to the gravity vector, with $y>0$ toward the lab ceiling. The $z$ axis is located along the mechanical chamber axis. $z>0$ is toward the antenna to form a right-handed coordinate system (see Fig. 2.8). In this coordinate system, the experimental domain is enclosed within $x \in[-1,1] \mathrm{cm}$, $y \in[-1,1.5] \mathrm{cm}$, and $z \in[-0.5,3.5] \mathrm{cm}$ for those locations between the boundary surface and the antenna. Fig. 2.9 shows the extent of the domain projected onto the boundary, along with the laser injection directions. The domain is determined in large part by the ability of the optical stages to translate between the HELIX magnets. No measurements were taken in the shadow of the boundary. Note that the laser injects toward $+\hat{x},-\hat{y}$, and $-\hat{z}$, so that in the raw data $\hat{v}_{x}\left\|\hat{x}, \hat{v}_{y}\right\|-\hat{y}$, and $\hat{v}_{z} \|-\hat{z}$. (In lab notes and some presentations, the $z$ axis follows the initially adopted convention that $\hat{z} \| \mathbf{B}$, so that $z>0$ is toward LEIA. This convention forms a left-handed system.) 

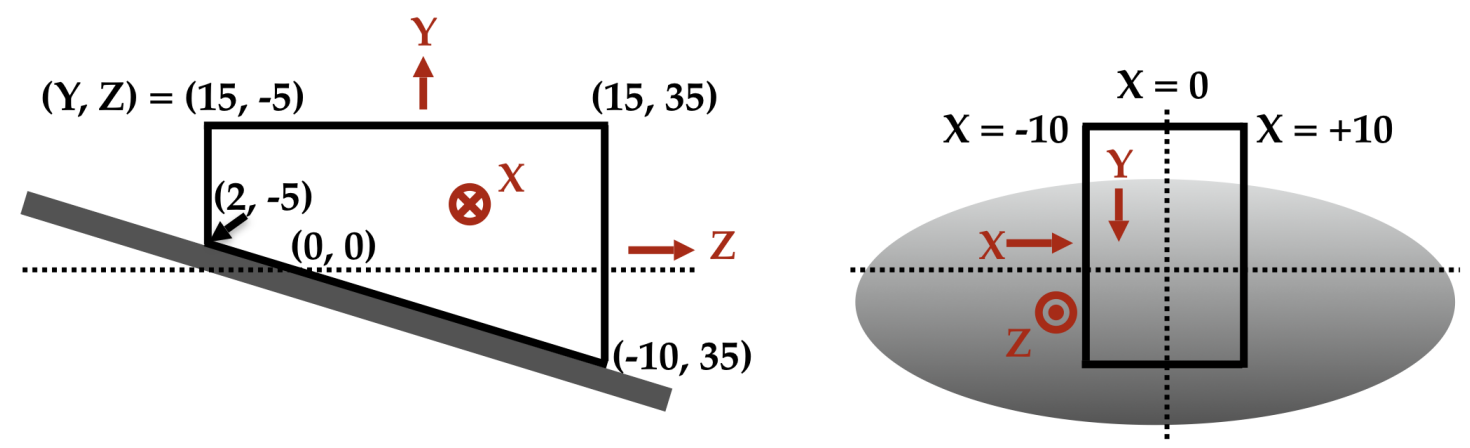

Figure 2.9: Experimental domain in the translational stage coordinate system. All coordinates are given in units of millimeters. Lettered arrows show the direction of laser injection.

The magnetic field intersects the boundary at an oblique angle. For the experiments reported here, the magnetic angle $\psi$ between the boundary normal and the magnetic field is $74^{\circ}$. The alignment of the boundary is achieved by injecting a collimated beam through the side port of HELIX and lowering the beam until the beam grazes the surface and the beam spot barely appears on the boundary edge. The stage is moved parallel to the chamber axis, toward the antenna, after which the beam is lowered again until it grazes the surface. If $\Delta z$ is the horizontal translation, and $\Delta y$ is the vertical drop, then the surface angle to the chamber axis, $\alpha=\pi / 2-\psi$, is

$$
\alpha\left[^{\circ}\right]=\frac{180}{\pi} \arctan \left(\frac{\Delta y}{\Delta z}\right) .
$$

For every $11 \mathrm{~mm}$ the stage moves along the axis, the vertical stage should drop $3 \mathrm{~mm}$, so that $\alpha=16.03^{\circ}$. The uncertainties $\delta(\Delta y)$ and $\delta(\Delta z)$ are estimated to be $\pm 0.5 \mathrm{~mm}$. The angle is measured over $33 \mathrm{~mm}$ - a $9 \mathrm{~mm}$ drop - so that if $q=\Delta y / \Delta z$, then $[86$, p. 61,65$]$

$$
\delta \alpha=\frac{180}{\pi}\left|\frac{d[\arctan (q)]}{d q}\right| \delta q=\frac{180}{\pi} \frac{\delta q}{q^{2}+1}=0.8^{\circ} .
$$

Therefore $\psi=74.0^{\circ} \pm 0.8^{\circ}$.

Ar-II LIF and Langmuir probe measurements were recorded at every location 


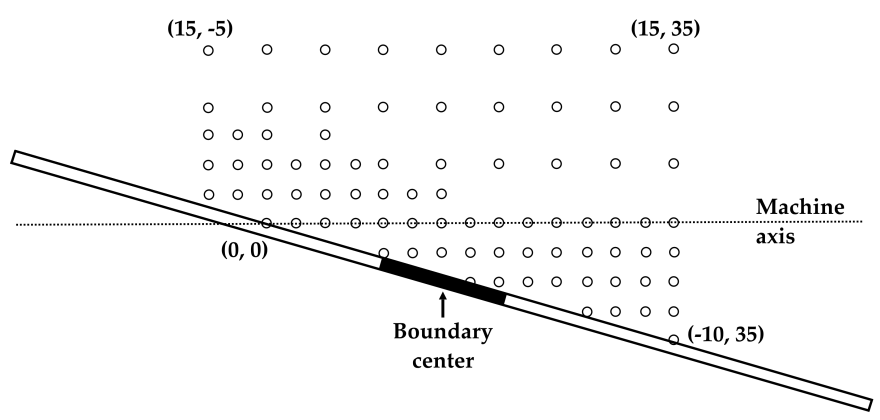

FIGURE 2.10: $y, z$ map of LIF and Langmuir probe measurement locations. Coordinates are given in millimeters. The location of beam dump at the center of the boundary is marked by a solid black box.

marked by a circle in Fig. 2.10. The locations were selected so that the laser beam, collection optics, and Langmuir probe tip would not overlap any two sample locations. Data were recorded at identical $(y, z)$ locations at $x \in\{0, \pm 0.5, \pm 1.0\}$ $\mathrm{cm}$. LIF data were recorded for $\hat{v}_{x}, \hat{v}_{y}$, and $\hat{v}_{z}$ directions, so that the velocity distribution and drift velocity vector were measured at each location in Fig. 2.10.

\subsubsection{Simulation coordinates}

Plasma physics models of the boundary region describe variation with respect to distance from an infinite plane. The coordinates are therefore determined by the surface normal vector, which is conventionally directed toward the surface. The lettering convention varies by author, but here the surface normal vector is referred to by $x$ and points at the surface. A right-handed coordinate system is established by setting $y$ along the surface, directed at an angle toward the lab ceiling. $z$ is along the boundary surface, directed at the lab doors (see Fig. 2.8).

The $z$ direction of the physics coordinate system is therefore identical to the $x$ direction of the translational stage system, and the other directions are rotated through the magnetic angle. If $\psi$ is the magnetic angle, between the magnetic field vector and the surface normal, then $\alpha=\pi / 2-\psi$. The coordinate systems can be 
transformed using

$$
\begin{aligned}
\bar{U}_{x}^{\operatorname{sim}} & =-\bar{U}_{y}^{\text {tran }} \sin (\psi)-\bar{U}_{z}^{\text {tran }} \cos (\psi) \\
\bar{U}_{y}^{\operatorname{sim}} & =\bar{U}_{y}^{\text {tran }} \cos (\psi)-\bar{U}_{z}^{\text {tran }} \sin (\psi) \\
\bar{U}_{z}^{\operatorname{sim}} & =\bar{U}_{x}
\end{aligned}
$$

where the sim superscript denotes the "simulation" coordinate system and tran denotes the translation stage, or "lab," system. Occasionally, it is more convenient to describe behavior aligned with the original lab system. Quantities in the lab system are marked with an asterisk, e.g., $x^{*}$. This coordinate system is shown alongside the simulation coordinate system in Fig. 2.8. Note that the $z$ direction is shared between the two systems such that $z^{*}=z$. 


\section{Chapter 3}

\section{Diagnostics}

Investigating plasma boundary interaction requires several specialized diagnostics and analysis methods. Fluid models are described by the bulk plasma fluid quantities - p, n, T, $\overline{\mathbf{u}}$ - and electromagnetic fields - E and B. Kinetic and gyrokinetic models, which provide greater physical detail, rely on information about the particle distributions. The primary diagnostics for determining the fluid quantities are pressure gauges and flow meters $(p)$, Langmuir probes $\left(n_{e} \approx n_{i} \approx n\right.$, and $\left.T_{e}\right)$, and laser-induced fluorescence $\left(T_{i}, \overline{\mathbf{u}}_{i}\right)$. The primary diagnostics for measuring the fields are gaussmeters $(\mathbf{B})$ and electrostatic probes $\left(\nabla \phi_{p} \rightarrow \mathbf{E}\right)$. Laser-induced fluorescence (LIF) offers ion and neutral particle velocity distribution information, while Langmuir probes provide the energy distributions of the electrons. These distribution measurements indicate where the fluid models exceed their limited applicability and help construct a more nuanced picture of the boundary environment. This chapter aims to provide a detailed description of the suite of diagnostics used in the plasma boundary experiments described in the rest of this document.

\subsection{Gaussmeter}

When present, magnetic fields strongly influence the plasma equilibrium. The special case of interacting boundary plasmas and magnetic fields is the focus of this 
project, and an accurate map of the magnetic field is required for experimental context and to establish a solid foundation for all that follows.

Magnetic fields in HELIX are measured using a Lake Shore Cryotronics Model 455 DSP gaussmeter [87, p. 1-1]. This gaussmeter measures $35 \mathrm{mG}$ to $350 \mathrm{kG}$ with $0.02 \mathrm{mG}$ resolution and $\pm 0.075 \%$ accuracy.

The gaussmeter has two components: a Hall effect sensor at the end of a probe and a digitization circuit. The circuit processes the probe signal and applies thermal compensation and background subtraction corrections. The field data can then be read out digitally through a serial port or recorded from the front display panel of the circuit unit. In these experiments, the latter method is employed. Readings from the display panel are recorded, confirmed by a second investigator, and transferred to a CSV, which is verified an extra time. Matching scans of the field map and copies of the CSV are preserved online and in several lab notebook records.

The active component in the Hall sensor is a rectangular InAs or GaAs semiconductor [87, p. 2-5]. The gaussmeter circuit drives current along one of the semiconductor axes. When the sensor is placed in a magnetic field, the electrons in the semiconductor experience a Lorentz force and drift perpendicularly to both the original current and the background field. This charge separation and buildup creates an electric potential between the two faces of the sensor, which has a known proportionality to the magnetic field:

$$
V_{B}=\gamma_{B} B \sin \theta
$$

where $V_{B}$ is the potential recorded by the circuit, in $\mathrm{V} ; \gamma_{B}$ is the proportionality constant, in $\mathrm{V} / \mathrm{G}$; $B$ is the magnetic field magnitude, in $\mathrm{G}$; and $\theta$ is the angle of incidence of the magnetic field onto the surface of the sensor. $V_{B}$ is maximized when the field is perpendicular to the sensor surface. The magnetic field is positive when it passes into the face of the axial probe or enters the face of the transverse probe on the side of the LakeShore label. Fig. 3.1 shows the two probe configurations and the direction of positive field with respect to each sensor. 

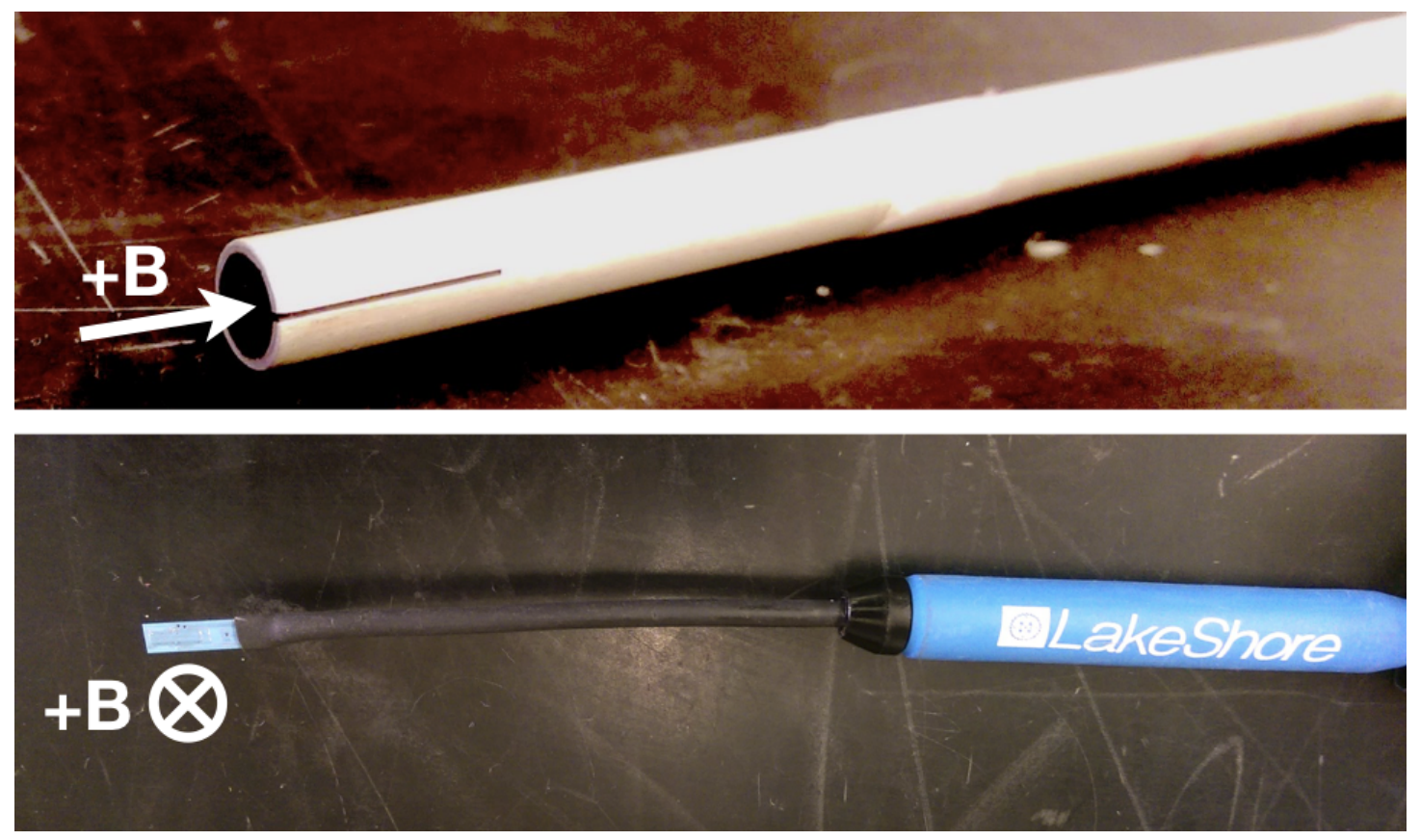

Figure 3.1: Axial (top) and transverse (bottom) gaussmeter probes indicating positive magnetic field direction.

Eq. (3.1) suggests a procedure for ensuring the accuracy of gaussmeter measurements. The transverse probe, which is held around its solid barrel by a rotational stage, is inserted into the open chamber. The probe should already be calibrated with the zeroing procedure outlined in the manual. The rotational stage is fixed to a tip-tilt mount to compensate for any small angle bend in the gaussmeter's flexible stalk. For stability, and to protect the fragile gaussmeter tip, the probe is used with the protective plastic cylinder in place whenever possible. Starting with the probe at the approximate orientation, and with the magnetic field on, the tip is tilted and rotated while maximizing or minimizing the field measurement on the display. The field is measured at several locations multiple times and the results are compared to confirm that the probe was not displaced at any time during measurement. The gaussmeter probe is held by the same stages that were used for the LIF optics, and initial positioning is confirmed by lasers, so that the locations of the magnetic field measurements are consistent with other diagnostics. 


\subsection{Langmuir probes}

Langmuir probes are among the oldest plasma diagnostics and provide measures of $n_{i}, n_{e}$ and $T_{e}$. Despite existing since the 1920s, when they were introduced by Langmuir [88] and Mott-Smith [89], no general probe theory exists, and probe fabrication and analysis techniques are still an active area of research. For decades, probe theory focused on quiescent, unmagnetized, low temperature plasmas. More recently, electrostatic probe techniques have extended probe applicability to more difficult regimes, such as exist in magnetized [90-94], radio-frequency [95, 96], and high temperature plasmas [29, 94, 97]. Several excellent and instructional reviews discuss Langmuir probe theory and application [27, 96, 98-101].

\subsubsection{Langmuir probe construction}

The need to perform 3D Langmuir probe measurements above the boundary necessitates the development of a novel probe translation mechanism. First, the entire probe and translation mechanism must retract behind a gate valve so that venting and pump-down is limited to a small volume, allowing maintenance or electrode exchange to occur rapidly. The retractability requirement sets a limit on the outer diameter of the probe translation mechanism, which must be smaller than the opening of the gate valve. Second, the effort to use off-the-shelf vacuum components whenever possible further constrains the design to only rotational motion and radial translation. That is, the probe could not be translated directly in Cartesian coordinates to match the coordinate systems of the other diagnostics, since that would require custom flanges, feedthroughs and fluorocarbon vacuum seals, which are expensive, awkward, and susceptible to leaks. Third, the need to keep the electrode orientation relative to the boundary surface the same at all sample locations excluded the use of ball-mount probes.

To meet these constraints, a new translation mechanism was designed that relies on a cylindrical coordinate system: radial $\rho$, rotational $\theta$, and vertical $y$. A rendering of the probe translation mechanism mounted to the top of the HELIX chamber 
is shown in Fig. 3.2, cut away to show the probe in place over the absorbing boundary. A photograph in Fig. 3.3 shows the probe electrode extending from the translation stage; the Macor cap is removed to reveal inner components of the Langmuir electrode. The probe electrode sits in a clamp mount, which is attached via a small post to a linear bearing stage. The small post slides along a closed groove in an arm located between the clamp mount and the bearing stage. As a $1 / 4$ " outer diameter (OD) drive shaft turns the arm, the groove in the arm moves the small post and linear bearing stage along a track, positioning the electrode at the desired radius $(\rho)$ from the center of a $21 / 2$ " OD stainless steel (SAE 316) disc. The disc is welded around a $1 / 2$ " OD tube that rotates (in $\theta$ ) the disc and radial mechanism and translates them vertically in the lab frame $(y)$. The $1 / 2$ " OD tube is fixed by the Ultra-Torr o-ring vacuum seals near the top of the translation assembly, concentric with the upper opening of the $6 "$ cross port on HELIX. The drive shaft passes through a rotational bearing in the central opening of the disc, continues through the entire length of the $1 / 2$ " OD tube, and emerges through an Ultra-Torr o-ring seal to air, where it can be turned independently from the rotation of the $1 / 2$ " OD tube around it.

The $y$ coordinate is shared with the coordinate system of the boundary and the laser optics. The $\rho$ and $\theta$ coordinates are determined by graded rotational stages fixed to the air side of the tube and drive shaft. These coordinates are then converted using software into the $x-z$ lab coordinate system. The probe position is confirmed by additional linear scales on the outside of the chamber and by laser sighting with the same optomechanical stages used for LIF to ensure consistent positioning across all diagnostics.

All mechanical components are fabricated from (SAE) Type 316 (non-L-type when possible) austenitic stainless steel. (Except for the expensive and difficult to acquire Type $316 \mathrm{~N}$ and $316 \mathrm{Ti}$ sub-varieties, Type 316 has the lowest magnetic permeability of the stainless steel metal group, especially after machining, annealing, and cold working [102, p. 21-23].) Type 316 stainless steels also exhibit lower secondary electron emission than aluminum [103-105]. The components of the translational mechanism that are not Type 316 are contained in the off-the-shelf linear stage and 


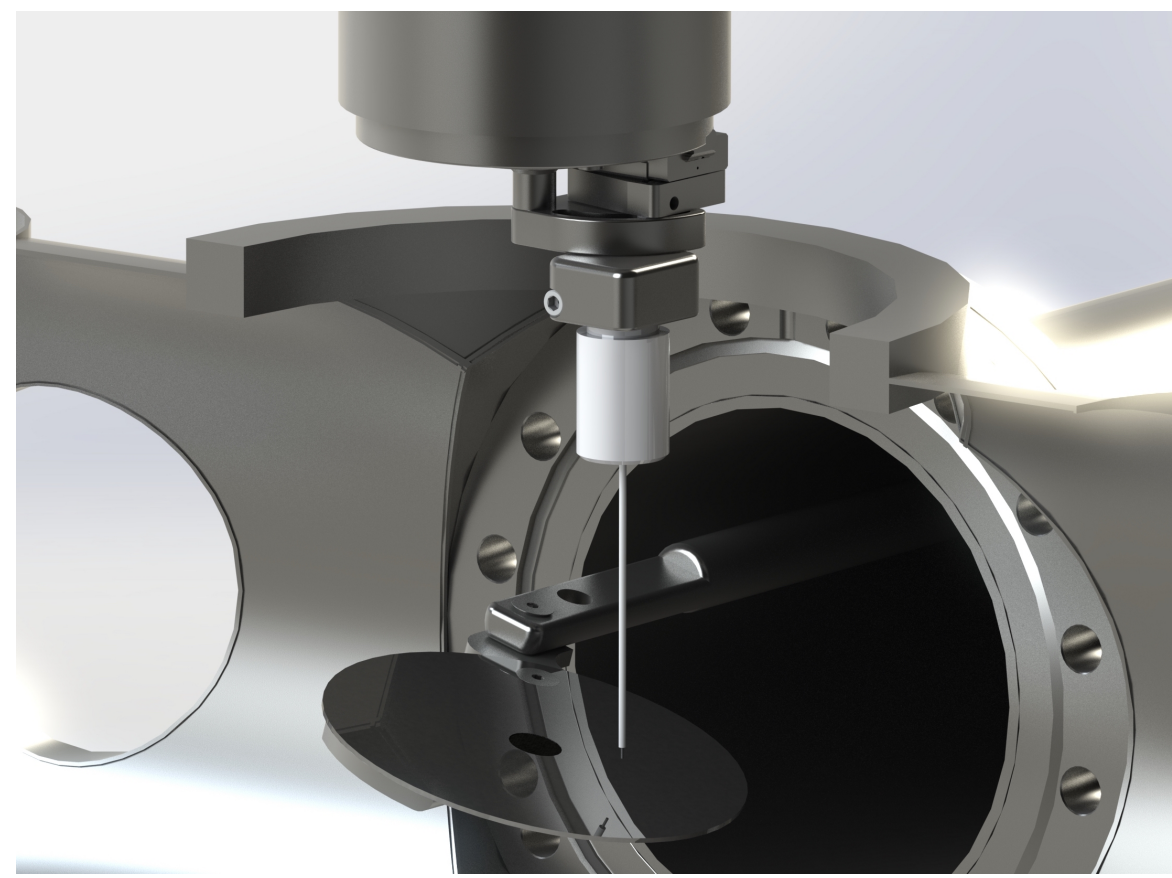

Figure 3.2: Cutaway of HELIX chamber showing 3D cylindrical coordinate translation stage in place over the boundary.

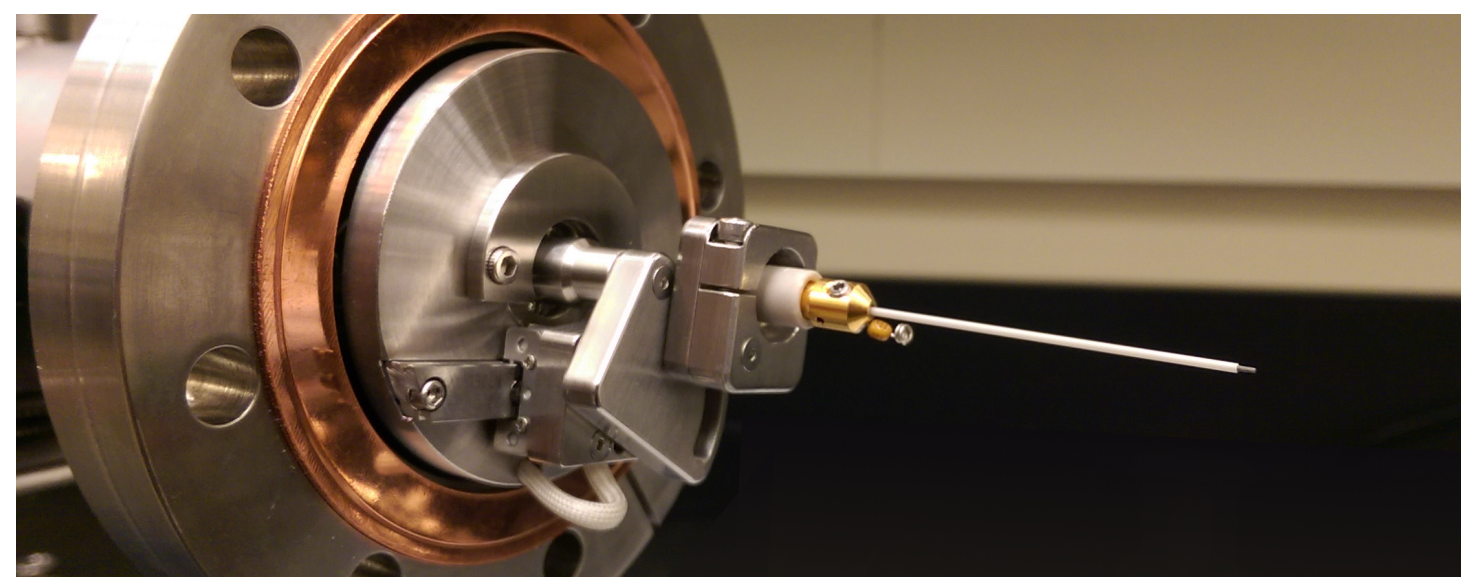

Figure 3.3: Photograph of the Langmuir probe electrode with the protective Macor cap removed to reveal probe tip internal components.

track, which are fabricated from magnetic Type 440 stainless steel. These pieces are small and are housed $>10 \mathrm{~cm}$ from the probe tip. Any magnetic perturbation from the presence of the probe is confirmed to be $<1 \%$ by gaussmeter measurement (see Fig. 2.7). Vented screws are used wherever trapped volumes would otherwise occur. 


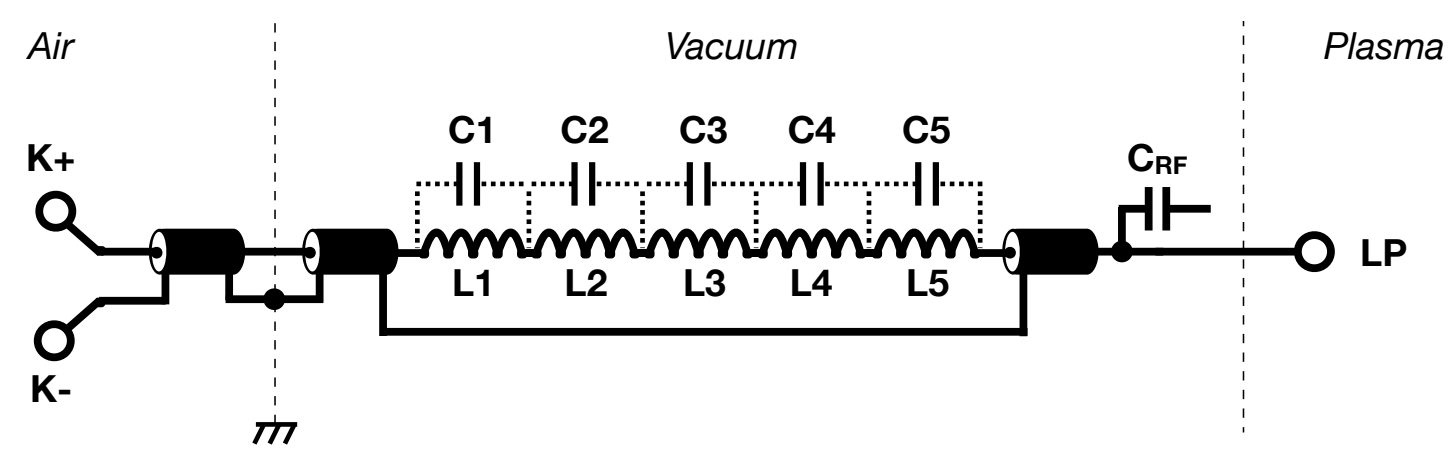

FiguRE 3.4: Langmuir probe circuit diagram. LP - Langmuir probe collection surface; $\mathbf{C}_{\mathbf{R F}}-\mathrm{RF}$ compensation electrode connected through $10 \mathrm{nF}$ tantalum capacitor; L1-L5 and accompanying capacitors - resonant inductors forming the choke chain; $\mathbf{L} 1-330 \mu \mathrm{H}, 6.8 \mathrm{MHz} ; \mathbf{L} 2-270 \mu \mathrm{H}, 7.3 \mathrm{MHz} ; \mathbf{L} 3-33 \mu \mathrm{H}$, $23 \mathrm{MHz}$; $\mathbf{4}-15 \mu \mathrm{H}, 47 \mathrm{MHz}$; $\mathbf{5}-33 \mu \mathrm{H}, 23 \mathrm{MHz}$; K+ and $\mathbf{K}-$ - the isolated terminals of the Keithley 2400 SourceMeter. The reference potential is provided by the chamber wall near the sample region.

\subsubsection{Langmuir probe circuit}

The probe electronics begin at the electrode immersed in the plasma. A schematic of the probe circuit is shown in Fig. 3.4. The Langmuir probe electrode is a $500 \pm 25$ um OD carbon tip, with $2.00 \pm 0.03 \mathrm{~mm}$ exposed from the end of a $1.00 \pm 0.03$ mm OD alumina $\left(\mathrm{Al}_{2} \mathrm{O}_{3}\right)$ shield. A set screw fixes the alumina inside the central bore of a brass connector. The end of the carbon electrode opposite to the plasmaimmersed collection surface is wrapped in a single strand of silver-coated copper wire. The wire-wrapped end is then soldered to the inside of the brass connector to form a good electrical contact between the carbon tip and the brass connector. A $10 \mathrm{nF}$ RF capacitor is soldered into a small hole drilled into the probe electrode side of the brass connector. The brass tip holder is press-fit onto a copper post isolated from the surrounding components by a polyether ether ketone (PEEK) disc. A PEEK cylinder shields the post and the sides of the brass tip holder from the surrounding stainless steel. A hollow Macor cap slides around the alumina shield, the brass tip holder, and the PEEK, and is clamped in place by a hinge in the stainless mount.

A 32 AWG silver-coated copper wire, wrapped around and soldered to the base of the copper post, transports the probe signal away from the probe electrode. The wire is insulated in a coaxial jacket of PTFE (Teflon), shielded by mesh braid 
conductor, and enclosed in Kapton film. The wire passes through the post that connects the clamp mount and the linear stage, continues through a groove on the underside of the linear stage mount plate, and passes through the translation mechanism disc. A small amount of wire remains on the plasma side of the disc to allow the stage to translate, but any exposed wire is shielded from the plasma by ceramic braid to insulate the wire and provide wear resistance.

On the side of the translation mechanism disc opposite to the translation mechanism, a small hose clamp keeps the ceramic braid-wrapped wire in place against the 1/2" OD stainless tube. An RF-compensation choke chain connects the wire from the electrode to the wire that carries the signal to the BNC vacuum feedthrough. (The RF compensation circuit is described below.) The diagnostic is easily converted from a Langmuir probe to a plasma heated emissive probe by exchanging electrode assemblies from carbon to thoriated tungsten and by removing the RF compensation. The wire between the compensation circuit and the BNC feedthrough is wound around the $1 / 2$ " OD stainless tube to allow the probe to translate. From the feedthrough, the signal passes to the Keithley 2400 SourceMeter via an RG-58 BNC cable.

The outer conductors of the BNC cable and Kapton coated coax wire are tied to instrumentation ground at the BNC feedthrough flange, shielding the signal path with low-impedance mesh braid from the Keithley up to the base of the probe electrode. These conductors and the grounded metallic probe tubes attenuate electromagnetic interference (EMI) which would otherwise appear as noise in the Langmuir probe signal. These components surround all but the very end of the probe circuit and provide a low-impedance path to ground for any EMI present outside of the plasma. The ground braid that surrounds the signal wire is unconnected from other grounded conductors at the probe electrode end to avoid ground loops.

The impedance of the probe circuit is important for several error sources inherent in electrostatic probe measurements (see Sec. 3.2.3.4). The resistance between the brass connector and the central conductor of the air-side BNC cable is measured 
by multimeter to be $\leq 11.6 \Omega$, where the contact resistance between the two multimeter probes is $0.2 \Omega$. The resistance between the clamp mount of the probe (the closest reference metallic surface to the probe tip) to a nearby vacuum flange is $\leq 0.6 \Omega$, indicating a good ground reference and suggesting strong EMI shielding. The resistance between the brass connector and the vacuum flange is beyond the range of the multimeter.

Some plasma conditions facilitate sputtering of the probe surface. High density plasmas such as helicon mode discharges in HELIX will rapidly sputter even tungsten probe surfaces, generating visible coatings of the alumina insulating surfaces within hours. If this sputtered surface is electrically connected to the probe tip, it becomes a vastly extended collection surface and often causes such severe error that any measurements must to be retaken with a new insulator. Two solutions are employed to avoid this problem. The first is to exchange the conductor with one that offers less sputtering yield. For argon, carbon electrodes exhibit dramatically less sputtering yield than tungsten, aluminum, copper, etc. The disadvantage of carbon is that it is brittle and cannot be wet by typical soldering methods. If a large enough (100s - 1000s of $\mu \mathrm{m})$ electrode is appropriate, a carbon electrode allows the probe to have a small diameter insulation shield that avoids perturbing the plasma. The second option [106] is to keep the insulating tip holder recessed inside an outer sleeve. If the tip holder is recessed sufficiently far back inside the outer sleeve, line-of-sight sputtering from the probe surface will collect on the inner surface of the sleeve, but a connection path will not be able to form between the sputtered surface and the probe electrode. This solution has the advantage of easing the requirement on the choice of electrode material and allows smaller electrodes, but it has the disadvantage that the assembly near the electrode is larger and may perturb the plasma.

For these measurements, a carbon electrode is used with a tightly fitted insulator. The resistance between the insulator near the electrode and the electrode surface is recorded after the measurements are complete. The resistances measured at several locations on the insulator, including its end, are out of range of the multimeter, indicating no collection area expansion due to sputtering occurred. 


\begin{tabular}{ccc}
\hline \hline Inductor & Inductance & Resonant Frequency \\
\hline L1 & $330 \mu \mathrm{H}$ & $6.8 \mathrm{MHz}$ \\
L2 & $270 \mu \mathrm{H}$ & $7.3 \mathrm{MHz}$ \\
L3 & $33 \mu \mathrm{H}$ & $23 \mathrm{MHz}$ \\
L4 & $15 \mu \mathrm{H}$ & $47 \mathrm{MHz}$ \\
L5 & $33 \mu \mathrm{H}$ & $23 \mathrm{MHz}$ \\
\hline \hline
\end{tabular}

TABLE 3.1: RF choke specifications used in Langmuir probe compensation circuit.

\section{RF compensation}

$\mathrm{RF}$ plasmas present an additional source of error from the influence of the RF power on the sheath around the probe. Several publications have discussed the problem in detail and have demonstrated the necessity of RF compensation [95, 101, 107, 108]. The solution used for Langmuir probe RF compensation in HELIX follows a modified design based on Sudit and Chen [107-110]. HELIX is unique in that its RF source can vary in frequency, and the probe must be able to compensate for sheath potential fluctuations from 8 - $14 \mathrm{MHz}$. For that reason, broad frequency $\mathrm{RF}$ compensation is implemented.

Fig. 3.4 shows the RF compensation circuit, which is comprised of a $10 \mathrm{nF}$ tantalum capacitor and five self-resonant inductors. One side of the capacitor is attached to the signal path of the probe at the base of the collection electrode. The other side is allowed to float, so that the local potential fluctuations due to the RF power are felt across the capacitor and absorbed. The self-resonant inductors are specialty RF chokes from Lenox-Fugle International [111] and are constructed so that the capacitance between the inductor and its central core creates a large impedance (typically 100s of $\mathrm{k} \Omega$ ) at specific RF-range resonance frequencies and their first, second and half harmonics. The values of the chokes are provided in Table 3.1.

The frequency response of the Langmuir probe is measured when the probe is disconnected from HELIX [112]: 


\begin{tabular}{cccc}
\hline \hline Quantity & Value & Quantity & Value \\
\hline Rate & $6.4 \mathrm{~Hz}$ & Start f & $100 \mathrm{~Hz}$ \\
Span & $9999900.00 \mathrm{~Hz}$ & Stop f & $10 \mathrm{MHz}$ \\
SWP CF & $5000050.0 \mathrm{~Hz}$ & Ampl. & $1 V_{p p}$ \\
Trig Src & SrcE-rate & Trig Rate & $10 \mathrm{~Hz}$ \\
\hline \hline
\end{tabular}

TABLE 3.2: DS345 settings for electrostatic probe frequency response measurements.

1. A Stanford Research Systems DS345 function generator is given the settings in Tab. 3.2.

2. The $1 V_{p p}$ output from the DS345 is split. One branch is attached to an oscilloscope. The other branch is connected to the probe tip $(+)$ and part of the vacuum assembly (-).

3. A ramp applied to the sweep input sweeps the frequency of the output signal from the start to stop frequencies defined in step 1.

4. The scope triggers on the sweep output from the rear panel of the DS345 and the horizontal axis scales by the set frequency range.

5. Plotting $20 \log _{10}\left(A / A_{\mathrm{DC}}\right)$ vs. the signal frequency generates a Bode plot, showing the frequency response of the probe, where $A_{\mathrm{DC}}$ is the $\mathrm{DC}$ amplitude (see Fig. 3.6).

The results of the frequency response test are shown in Fig. 3.5 and Fig. 3.6. The effect of the RF compensation is clear when comparing the output responses of the two probes: the Langmuir probe, which contains the RF compensation, exhibits a $-3 \mathrm{~dB}$ (50\% attenuation) frequency at $\approx 25 \mathrm{kHz}$, whereas the emissive probe only begins to attenuate near $10 \mathrm{MHz}$. 

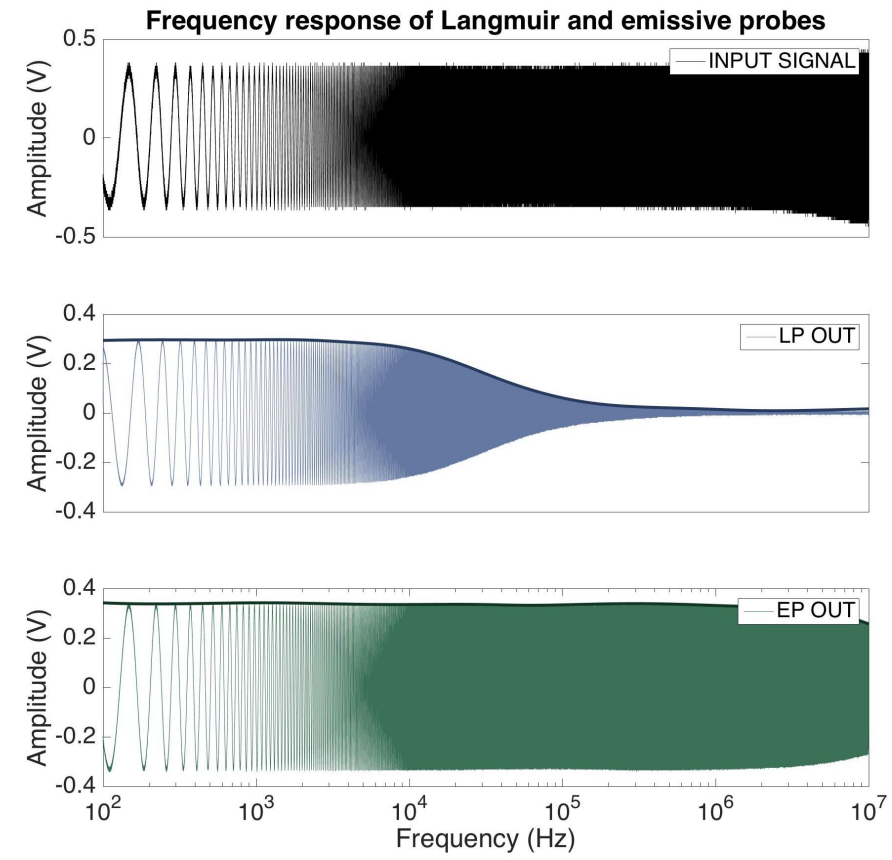

FIgURE 3.5: Input and output frequency sweeps for Langmuir and emissive probes.

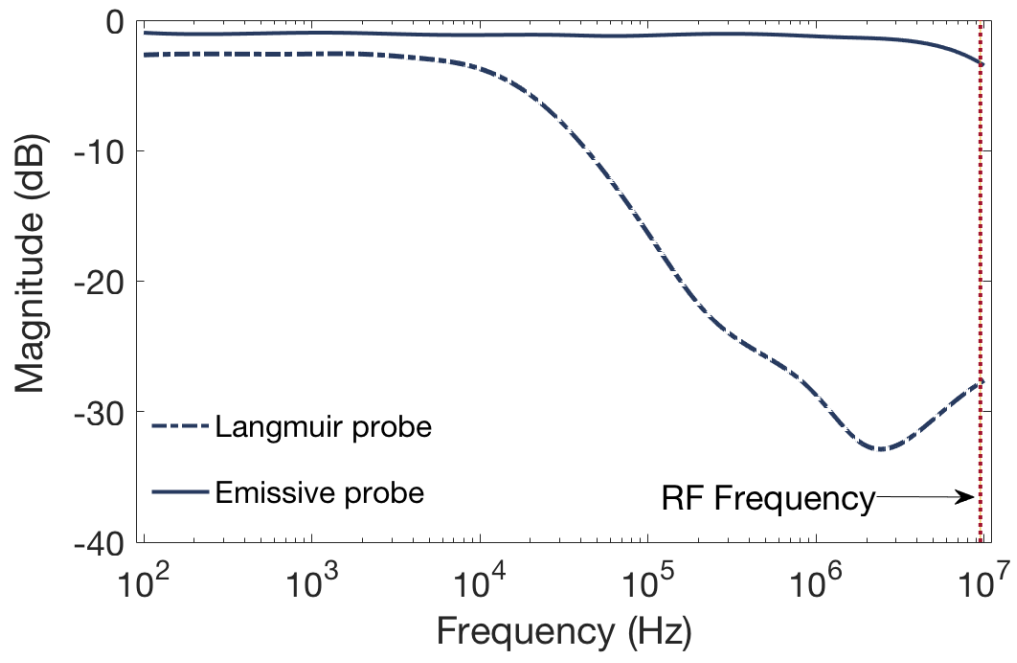

FiguRE 3.6: Bode plot for the Langmuir and emissive probes, showing effect of RF compensation.

\subsubsection{Langmuir probe analysis}

\subsubsection{Introduction}

Most analyses of Langmuir probe data begin with a plot showing the current $I$ drawn from the plasma into the probe as a function of the potential $V$ applied between the probe and a reference potential for the plasma, often set at the chamber 
wall. These plots are referred to as $I-V$ traces or characteristics. Probe characteristics are broken down into three sections, delineated by a pair of voltages: the floating potential $\phi_{f}$ and the plasma potential $\phi_{p}$. The floating potential is the bias voltage applied to the probe at which the electron and ion currents are equal and the net current collected by the probe is zero. The plasma potential is the potential that exists in the space between the charged particles of the plasma.

The three sections are treated separately:

Ion saturation region $-V \ll \phi_{p}$ : When the potential applied to the probe is driven very negative $\left(V \lesssim \phi_{p}-6 T_{e}\right.$ ) relative to the plasma potential the ions are attracted to the probe surface and collected while the electrons are almost completely repelled. In this region, the probe current consists primarily of plasma ions. In some circumstances, the probe draws no more current despite being driven to more negative potentials. This region is referred to as ion saturation, regardless of the actual saturation of the ion current.

Electron saturation region $-V \gg \phi_{p}$ : When the bias potential is greater than the plasma potential the current collected by the probe is dominated by electrons. This region is therefore referred to as electron saturation.

Transition region $-\phi_{f}<V<\phi_{p}$ : The region of the characteristic roughly between the floating and plasma potentials is referred to as the electron retarding or transition region. In this region both species are collected. When the energies of the plasma electrons are Maxwellian, the collected current in the transition region increases exponentially with increasing voltage.

Figure 3.7 shows the three regions of a Langmuir probe characteristic for a typical helicon source plasma measured in HELIX (IV\#16 from 17 April 2017). The three regions are identified in the figure.

The hazard of Langmuir probe analysis is that the deceptive simplicity of a probe's construction conceals the assumptions behind the design and choice of analysis method, which in any case had to be chosen before measuring the plasma on 


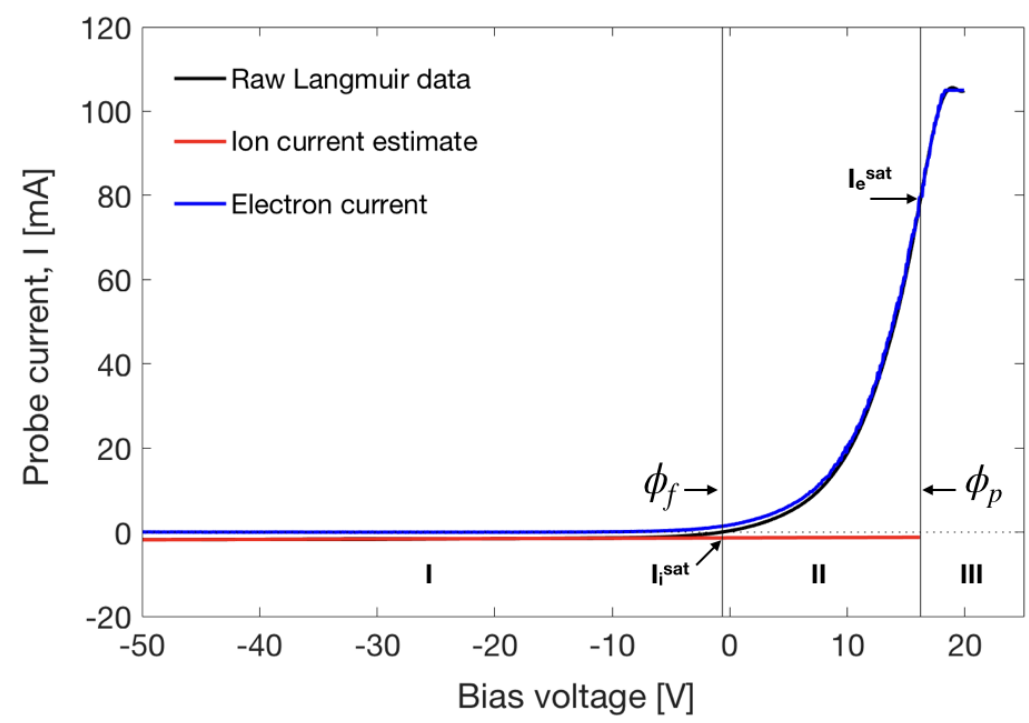

FiguRE 3.7: Probe characteristic showing: I. ion saturation, II. transition region, III. electron saturation. The current is limited to $100 \mathrm{~mA}$ by instrumentation.

which those assumptions are based. The researcher is forced to make a strong ansatz (or several), work through the appropriate analysis, and then verify the validity of the choices made.

\section{Reference data}

The reference data appearing throughout this section is taken from characteristic IV\#16 recorded on 17 April 2017. This characteristic was recorded $1.5 \mathrm{~cm}$ above an absorbing boundary, at the horizontal center of the $6 "$ cross port on HELIX, in a $B=600 \mathrm{G}, 20 \mathrm{sccm}, 3.6 \mathrm{mTorr}$ (end feed), $P_{\mathrm{rf}}=650 \mathrm{~W}, f_{\mathrm{rf}}=9.5 \mathrm{MHz}$, 99.999\% argon helicon plasma.

Noise in the current data often requires digital smoothing, which can introduce error and distortion to the characteristic if applied incorrectly. A description of the smoothing techniques used at several steps during the analysis of each characteristic is described in Appendix A. 


\subsubsection{Calculation of the fluid quantities $n_{i}, n_{e}$, and $T_{e}$}

This section reviews several techniques for determining the plasma fluid properties $n_{i}, n_{e}$, and $T_{e}$ from Langmuir probe data. $T_{i}$ is very difficult to obtain accurately using electrostatic probes, because of the difficulty of separating the ion and electron contributions [27]. To measure $T_{i}$ with a probe one should use a baffled or ion sensitive probe, or use a retarding field energy analyzer. These diagnostics are all perturbative and LIF is a preferable diagnostic for measuring the ions.

\section{Conventional Langmuir analysis}

Conventional analysis, as introduced by Langmuir and Mott-Smith [88], assumes that the electrons in the bulk plasma away from the probe are isotropic - particles approach the probe surface with angular symmetry — and that their energies are described by a Maxwellian distribution. Following Chen [101] and Hershkowitz [98] the total current is a combination of the net current from the ions and electrons, such that $I=I_{e}-I_{i}$. In the transition region,

$$
I_{e}(V)=I_{e}^{\text {sat. }} \exp \left[-e\left(\phi_{p}-V\right) / k_{B} T_{e}\right]
$$

where $I_{e}^{\text {sat. }}$ is the electron saturation current at the plasma potential, in amps; $k_{B} T_{e}$ is the electron temperature, the width of the Maxwellian energy distribution, in $\mathrm{eV} ; k_{B}$ is Boltzmann's constant; and $e$ is the elementary charge, $1.602 \times 10^{-19}$ C. The currents are modeled by the particle flux to the probe surface such that $J_{\sigma}=n_{\sigma} e v_{\sigma}=I_{\sigma} / A_{p}$, where $J$ is the current density at the probe surface, in $A / m^{2} ; n$ is the particle density, in $m^{-3} ; A_{p}=\pi r_{p}^{2}+2 \pi r_{p} \ell_{p}$ is the area of the single-cap cylindrical probe, in $m^{2}$, where $r_{p}$ and $\ell_{p}$ are the probe radius and exposed length, respectively; $v$ is the velocity of the incident particles, in $\mathrm{m} / \mathrm{s}$; and $\sigma$ denotes the particle species, either electron or ion. If these particle fluxes stabilize in the saturation regions, the electrons approach at their thermal velocity $\left(v_{\text {the }}=\sqrt{8 T_{e} / \pi m_{e}}\right)$ and the ions are incident at the Bohm velocity [6], $v_{B i}=$ 
$\sqrt{T_{e} / m_{i}}$. The two saturation currents are related to the plasma parameters via

$$
\begin{aligned}
I_{e}^{\text {sat. }} & =\frac{n_{e} e A_{p}}{4}\left(\frac{8 k_{B} T_{e}}{\pi m_{e}}\right)^{1 / 2} \\
I_{i}^{\text {sat. }} & =\alpha^{\prime} n_{i} e A_{p}\left(\frac{k_{B} T_{e}}{m_{i}}\right)^{1 / 2} .
\end{aligned}
$$

Here $m_{i}$ and $m_{e}$ are the ion and electron masses, in $\mathrm{kg}$, and $\alpha^{\prime}$ arises from an estimate of the variation in the ion density between the probe surface and the plasma far from the perturbation region around the probe. $\alpha^{\prime}$ depends on the geometry of the probe and on the local Debye length $\lambda_{D}$ through the empirical relation [100]:

$$
\alpha^{\prime}=0.607+2432 / \exp \left[7.01\left(r_{p} / \lambda_{D}\right)^{0.096}\right]
$$

where

$$
\lambda_{D}=\left[\frac{\epsilon_{o} k_{B} / e^{2}}{n_{e} / T_{e}+n_{i} / T_{i}}\right]^{1 / 2}
$$

Here $\epsilon_{o}$ is the permittivity of free space and $T_{i}$ is the ion temperature. Typically, $\alpha^{\prime}$ is estimated as $\alpha_{o}=\exp (-1 / 2) \approx 0.61$. For the more complicated magnetized plasmas in helicons, it may be significantly higher. In HELIX, Eq. (3.5) and typical plasma quantities suggest that a closer estimate is $\alpha^{\prime}=0.8 \pm 0.1$. If high accuracy is required, it is often necessary to guess a value for $\alpha^{\prime}$, compute $n$ and $T_{e}$, calculate $\alpha^{\prime}$ using Eq. (3.5) and (3.6), and iterate until self-consistent values for $\alpha^{\prime}, n$, and $T_{e}$ are obtained.

$T_{e}$ is determined from the transition region, since Eq. (3.2) gives

$$
T_{e}=\left[\frac{d \ln \left(I_{e}(V)\right)}{d V}\right]^{-1}
$$

which is determined directly from the slope of the semilog plot $\ln \left(I_{e}\right)$ vs. $V$. The electron saturation current is measured at the plasma potential and the ion current is determined from a linear fit to the ion region extrapolated back to the floating 
potential. The densities are then computed by reorganizing Eqs. (3.3) and (3.4):

$$
\begin{gathered}
n_{e}=\frac{\left(2 \pi m_{e}\right)^{1 / 2}}{e A_{p}} \frac{I_{e}^{\text {sat. }}}{\left(k_{B} T_{e}\right)^{1 / 2}} \\
n_{i}=\frac{m_{i}^{1 / 2}}{\alpha^{\prime} e A_{p}} \frac{I_{i}^{\text {sat. }}}{\left(k_{B} T_{e}\right)^{1 / 2}} .
\end{gathered}
$$

In summary, the procedure for the conventional method is

1. Fit a line to the ion region of a plot of $I$ vs $V$, emphasizing the region at high negative potential where the electron contribution to the current may be neglected;

2. Isolate the electron current by calculating $I_{e}=I-I_{i}$;

3. Fit a line to the semilog plot of $\ln \left(I_{e}\right)$ vs. $V$ to obtain $T_{e}$;

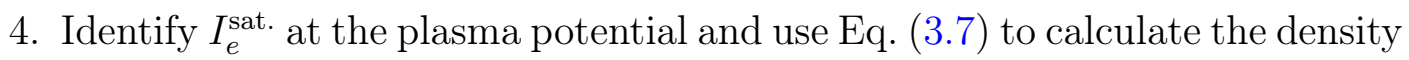
from the electron current

and/or identify $I_{i}^{\text {sat. }}$ from the ion current extrapolated to the floating potential and use Eq. (3.8) to calculate the density from the ion current.

In Fig. 3.7, step 1 is shown by the small current red line, and the result of step 2 is the blue curve showing the isolated electron current. The saturation currents in step 4 are identified as well. Step 3, finding $T_{e}$, is demonstrated in Fig. 3.8.

Accurately determining the density from the electron saturation current presents several difficulties. First, it is not always clear exactly where the plasma potential is, and a significant amount of error can be introduced from the exponential dependence on $\phi_{p}$. Fig. 3.7 identifies the plasma potential at the inflection point of the curve, a method preferred in several published accounts [27, 96], whereas others prefer to identify a knee [98], which would be at least above $V \approx 18 \mathrm{~V}$ in Fig. 3.7, where the current protection circuit has been activated. This choice alone results in a $20 \%$ difference in density. Many probe characteristics never achieve electron saturation, where the geometry of the probe, magnetic fields, noise, and 


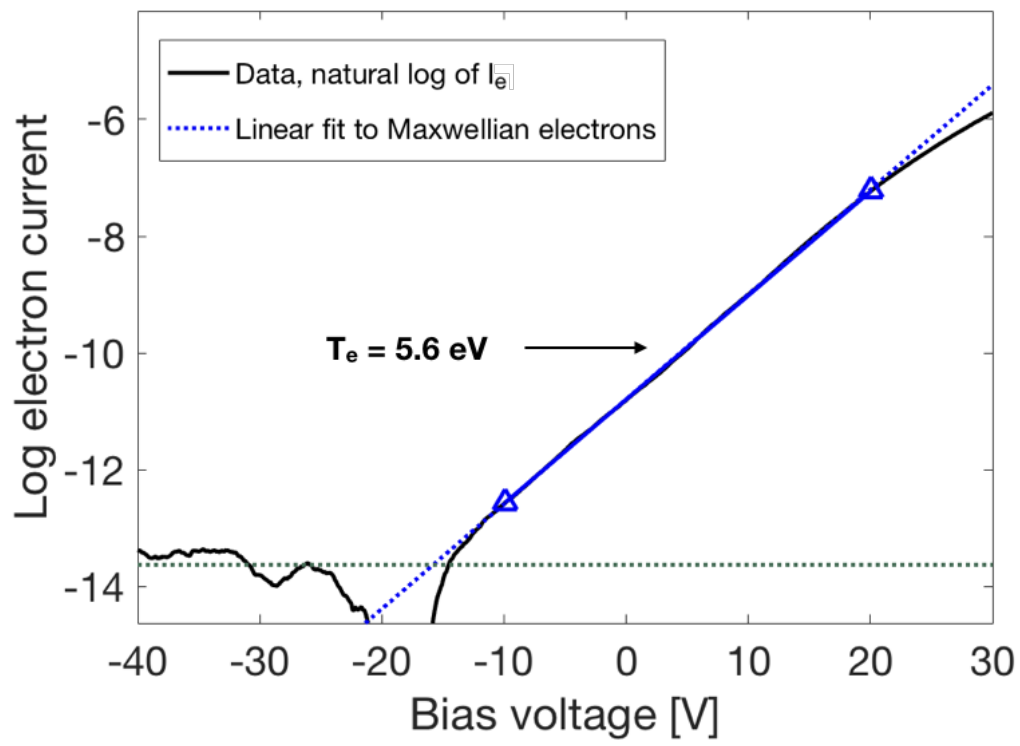

FIgURE 3.8: Semilog plot of the electron density dependence on probe voltage. Data is shown in solid black. A linear fit giving the inverse electron temperature is in dotted blue. The excellent linear fit is evidence that these electrons are Maxwellian. The dotted horizontal line marks the noise floor for the characteristic.

distribution of the electrons affect the shape of the characteristic [98]. For these reasons, the ion region is often used to measure plasma density.

Popular analyses using the ion region of the characteristic fall into two main genres of analysis. (This discussion closely follows the review by Godyak and Alexandrovich [113].) The appropriate analysis is determined by the unitless parameter $r_{p} / \lambda_{D}$, denoted $\xi_{p}$ in the notation of Ref. [100].

\section{The BRL and ABR analyses}

The first method assumes that positive ions approach the probe surface along radial trajectories, implying that the probe is larger than the Debye length, i.e., $\xi_{p}>1$. The "thin sheath" method for these situations is referred to as ABR, after Allen, Boyd, and Reynolds [114] who first proposed it. ABR was extended to cylindrical geometries by Chen [100]. The disadvantage of ABR analysis is that it involves the generation of many parametric curves which are then compared to the data. Calibration of ABR against non-perturbative diagnostics found that 
ABR consistently underestimates plasma density because it ignores the non-radial trajectories ions make on their approach [115].

Another theory, which incorporates ion orbits and finite sheaths, was introduced by Bernstein, Rabinowitz, and Laframboise [100, 116, 117] and is referred to as the BRL method. Calibration of BRL against non-perturbative diagnostics found that BRL consistently overestimates plasma density because it ignores collisions in the sheath that reduce the angular momentum of passing ions, increasing the ion current. The geometric mean of the ABR and BRL densities is considered fairly good, however both techniques are computationally difficult and are usually avoided.

\section{Orbital motion limited theory}

An alternative method, referred to as orbital motion limited theory and abbreviated OML, follows early work by Mott-Smith and Langmuir [89]. (This discussion closely follows Chen et al. [115].) OML posits that ions with large angular momentum will pass the probe and not be collected, whereas ions with small enough angular momentum will be attracted by the probe potential and collected. In this case, the probe current is [115]

$$
I_{i}(V)=A_{p} n_{\mathrm{OML}} e \frac{\sqrt{2}}{\pi}\left[\frac{e\left(\phi_{p}^{\mathrm{OML}}-V\right)}{m_{i}}\right]^{1 / 2}
$$

where $I_{i}$ is the ion current in amps, $n_{\mathrm{OML}}$ is the bulk ion density in $m^{-3}$, and $\phi_{p}^{\mathrm{OML}}$ is a proxy plasma potential to be described shortly. An immediately evident benefit of the OML method in Eq. (3.9) is that it does not require an accurate measure of $T_{e}$. Rearranging Eq. (3.9) and evaluating it at $V=\phi_{f}$ gives

$$
n_{\mathrm{OML}}=C_{\mathrm{OML}} \frac{I_{i}\left(\phi_{f}\right)}{\left[\phi_{p}^{\mathrm{OML}}-\phi_{f}\right]^{1 / 2}} ; \quad C_{\mathrm{OML}} \doteq \frac{\pi}{A_{p}} \sqrt{\frac{m_{i}}{2 e^{3}}}
$$

According to Eq. (3.9) the squared ion current is linear in $V$. The electron current $I_{e}$ is obtained by subtracting the linear fit of $I^{2}$ from the total measured probe 


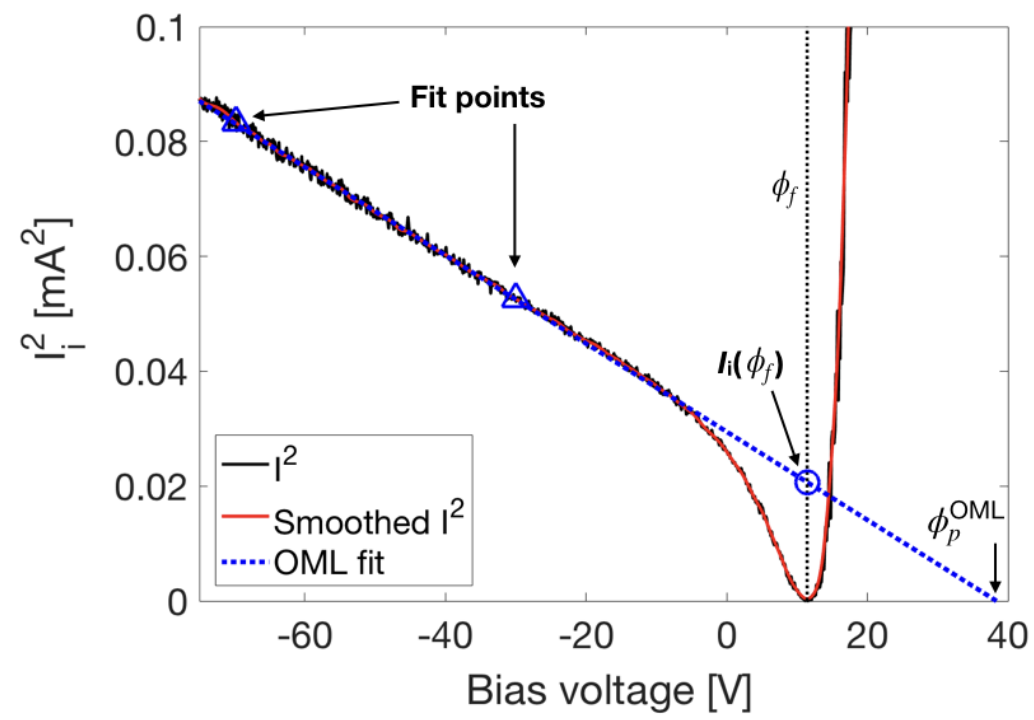

Figure 3.9: Example $I^{2}-V$ plot used in OML analysis. Data are shown in solid black; smoothed data are shown in red. Extrapolated ion current is shown in dashed blue

current. This current is described by

$$
I_{e}(V)=n e v_{t h e} e^{e\left(V-\phi_{p}\right) / k_{B} T_{e}} .
$$

$T_{e}$ can be calculated from the slope of the semilog plot of $\ln \left(I_{e}\right)$ as in conventional Langmuir analysis.

If OML is valid, a linear fit to $I^{2}-V$ produces a better fit than $I-V$. (The validity condition of OML is described in detail in Refs. [118] and [119] and should be verified for each characteristic.) A demonstration of a typical OML analysis is shown in Fig. 3.9. The procedure for OML analysis is

1. Plot $I^{2}-V$. Identify $\phi_{f}$ at the minimum of the curve where $I=0$.

2. Fit a line to the ion current region, where the voltage is highly negative.

3. Extrapolate this line to find $\phi_{p}^{\mathrm{OML}}$ and $I_{i}\left(\phi_{f}\right)$.

4. Calculate $n_{\mathrm{OML}}$ using Eq. (3.10).

5. Subtract the line (found in $\# 2$ ) from the total current to isolate $I_{e}$.

6. Find $T_{e}$ from the slope of the semilog plot of $I_{e}-V$ using Eq. (3.11). 
7. Calculate $\phi_{p}$ from $I_{e}^{\prime \prime}\left(\phi_{p}\right)=0$.

Of the thin sheath theories, OML densities are observed to be surprisingly accurate given the questionable validity of the analysis theory [115].

\section{Child-Langmuir floating point analysis}

OML is considered to be appropriate only when $\xi_{p} \lesssim 1$, and Chen et al. [120] propose an additional technique that eases this requirement which they refer to as the Child-Langmuir floating point method, abbreviated CL- $V_{f}$. A brief version of their description of the technique is given here. They observe that at higher densities - in the $10^{16}-10^{18} \mathrm{~m}^{-3}$ range relevant to HELIX plasmas, specifically - a linear fit to the ion current of $I^{4 / 3}-V\left(\mathrm{CL}-V_{f}\right)$ is better than a fit to the ion current of $I^{2}-V(\mathrm{OML})$ or $I^{(>2)}-V(\mathrm{ABR})$. The ions are collected by a Bohm sheath, as in Eq. (3.4). The collection area is defined not by the physical probe surface but by the Child-Langmuir sheath that forms around it, such that Eq. (3.4) becomes

$$
I_{i}=\alpha_{o} n A_{s h} e\left(k_{B} T_{e} / m_{i}\right)^{1 / 2}
$$

resulting in a density of

$$
n=\frac{I_{i}}{e \alpha_{o} A_{s h}}\left(\frac{m_{i}}{k_{B} T_{e}}\right)^{1 / 2},
$$

where the sheath area $A_{s h}$ now includes the sheath geometry. (Note that in the description of this technique by Chen et al. the current in Eq. (3.12) is given without the $e$ and has units of particles/second, not amps. I keep the $e$ here.) Current collected through the end cap of the probe is estimated at $<5 \%$ and is neglected. The CL- $V_{f}$ technique follows the algorithm:

1. Determine $\phi_{p}$ at $\max (d I / d V)$.

2. Fit a line to the ion region of a plot of $I^{4 / 3}-V$.

3. Extrapolate the linear fit to the floating potential $\phi_{f}$ to obtain $I_{i}\left(\phi_{f}\right)$.

4. Subtract $I_{i}^{4 / 3}$ to isolate $I_{e}$. 
5. Fit a line to $\ln \left(I_{e}\right)$ vs $V$ to obtain $T_{e}$.

6. Use $T_{e}$ to calculate $\eta(V) \doteq-\left(V-\phi_{p}\right) / k_{B} T_{e}$ and $\eta_{f}=\eta\left(\phi_{f}\right)$.

7. Calculate $n$ using the Child-Langmuir sheath thickness and the derived quantities $\eta_{f}$ and $T_{e}$.

With $\eta_{f}$ and $T_{e}$ in hand, $n$ is determined by the Child-Langmuir sheath area, $A_{s h}$ via the replacement $r_{p} \rightarrow r_{p}+d$, where $d$ is the sheath thickness per the Child-Langmuir formula [121, p. 165]:

$$
d=\frac{1}{3} \sqrt{\frac{2}{\alpha_{o}}}\left(2 \eta_{f}\right)^{3 / 4} \lambda_{D}=\frac{1}{3} \sqrt{\frac{2}{\alpha_{o}}}\left(2 \eta_{f}\right)^{3 / 4}\left(\frac{\epsilon_{o} k_{B} T_{e}}{e^{2}}\right)^{1 / 2} n^{1 / 2}
$$

Incorporating this substitution into Eq. (3.12) produces a quadratic equation for $n^{1 / 2}$ :

$$
n=\frac{1}{4 A^{2}}\left[-B \pm\left(B^{2}+4 A C\right)^{1 / 2}\right]^{2}
$$

where

$$
\begin{aligned}
& A \doteq r_{p} \\
& B \doteq \eta_{f}^{3 / 4}\left(\epsilon_{o} k_{B} T_{e} / e^{2}\right)^{1 / 2} \\
& C \doteq \frac{m_{i}^{1 / 2}}{2 \pi \ell_{p} \alpha_{o} e} \frac{I_{i}\left(\phi_{f}\right)}{k_{B} T_{e}}
\end{aligned}
$$

Comparisons of the results from the methods mentioned here have been published by several research groups $[113,115,120]$. Chen et al. find that, for RF plasmas created in 1-10 mTorr of argon, densities calculated using the Child-Langmuir floating point method are in excellent agreement with non-perturbative microwave measurements for RF powers between 300 and $900 \mathrm{~W}$. ABR and electron saturation densities are observed to be about a factor of 2 lower [101]. 


\subsubsection{EDFs, EEDFs and EEPFs}

The majority of the fluid quantity analyses assume that the electron energies are Maxwellian and that the ions, too, are Maxwellian or are very cold, such that $T_{i} \rightarrow 0$. These assumptions are frequently invalid, and many times deviations from the Maxwellian distribution offer insights into underlying physics that conventional methods pass over. The difficulty lies in trying to recover the bulk distribution in the unperturbed region of the plasma accurately while recognizing that we are only able to measure particles that have passed through the perturbation region and reached the probe surface.

(This discussion follows the discussion in Ref. [27] closely.) The kinetic approach to the problem attempts to solve the Vlasov-Boltzmann equation

$$
\frac{\partial f}{\partial t}+\mathbf{v} \cdot \nabla_{x} f+\frac{e}{m_{e}}(\mathbf{E}+\mathbf{v} \times \mathbf{B}) \cdot \nabla_{v} f=S
$$

where $f(\mathbf{r}, \mathbf{v}, t)$ is the electron distribution function $(\mathrm{EDF}), \mathbf{E}$ and $\mathbf{B}$ are the electric and magnetic fields, and $S$ represents any sources or sinks. At equilibrium, the problem turns to determining how the EDF at the probe surface $f\left(\mathbf{r}_{s}, \mathbf{v}_{s}\right)$ is related to the EDF far from the region perturbed by the probe $f(\mathbf{r}, \mathbf{v})$ by making approximations about collisions, i.e., about $S$, and by making assumptions about the shape of $\mathbf{E}(\mathbf{r})$. The unperturbed $f(\mathbf{r}, \mathbf{v})$ is related through $f\left(\mathbf{r}_{s}, \mathbf{v}_{s}\right)$ to the current density entering the probe as a function of the repulsive potential.

Cylindrical Langmuir probes do not discern the approach direction of the particles they collect, but they do discern the energies of those particles since the electron kinetic energy must be sufficient to overcome the repulsive Coulomb potential of the probe. The speed $v$ and kinetic energy $\varepsilon$ are related through the thermal speed $v=\sqrt{2 \varepsilon / m_{e}}$. It is possible to construct the electron energy distribution function (EEDF), written $f_{e}(\mathbf{r}, \varepsilon)$, or the electron energy probability function (EEPF), written $f_{p}(\mathbf{r}, \varepsilon)$, that in isotropic plasmas contain the same information about the electrons near the probe as the EDF. Conversion between them is simple: $\left(4 \sqrt{2} \pi / m^{3 / 2}\right) f_{o}(\varepsilon)=f_{p}(\varepsilon)=f_{e}(\varepsilon) / \sqrt{\varepsilon}$. 
Once the EEDF or EEPF is constructed, the fluid quantities are derived from the energy moments. The distribution is normalized to the electron density, so that the $0^{\text {th }}$-moment is

$$
n_{e}=\int_{0}^{\infty} f_{e}(\varepsilon) d \varepsilon=\int_{0}^{\infty} \varepsilon^{1 / 2} f_{p}(\varepsilon) d \varepsilon
$$

For electron energy measured in $\mathrm{eV},\left[f_{e}(\varepsilon)\right]=\mathrm{eV}^{-1} \mathrm{~m}^{-3}$ and $\left[f_{p}(\varepsilon)\right]=\mathrm{eV}^{-3 / 2} \mathrm{~m}^{-3}$ [96]. The temperature is defined relative to the mean energy of the distribution (instead of by the distribution width for Maxwellian electrons) such that the $1^{\text {st }}$ moment of the energy distribution provides the effective temperature

$$
T_{\text {eff }} \doteq \frac{2}{3}\langle\varepsilon\rangle=\frac{2}{3}\left[\frac{1}{n_{e}} \int_{0}^{\infty} \varepsilon f_{e}(\varepsilon) d \varepsilon\right]=\frac{2}{3}\left[\frac{1}{n_{e}} \int_{0}^{\infty} \varepsilon^{3 / 2} f_{p}(\varepsilon) d \varepsilon\right]
$$

More on distribution functions can be found in Refs. [27, 96, 113, 121] and [122].

Note: many authors are casual in labeling EDFs, EEDFs and EEPFs correctly in their work, often do not declare their units, and propagate many typos. It is easy to find examples of EDFs, EEDFs, and EEPFs mislabeled or interchanged within a single paragraph without notice. Absent or superfluous $e$ 's and $m_{e}$ 's occur in published equations and propagate through references. Therefore, the reader is advised to verify these functions by their units before using them in calculations to avoid extra factors of $10^{-19}$ or $10^{-31}$.

\section{Scale length approximations of the Vlasov-Boltzmann equation}

Many of the approximations made by various attempts to solve Eq. (3.15) are summarized by Demidov et al. in Ref. [27] and by Godyak and Demidov in Ref. [96]. These approximations arise from comparing various lengths that govern the plasma region around the probe. Some methods are drastically more complicated than others. If one is fortunate enough to have foreknowledge of the plasma conditions and can tabulate the various lengths in advance, it is usually possible to alter the geometry of the probe electrode and to secure a simpler analysis. 
As a guiding principle, an interaction may be omitted from the analysis if its scale length for a particle species $\lambda$ is much larger than the characteristic probe dimension $d$ and the sheath width $h$, i.e., when $\lambda \gg d+h$. For cylindrical probes,

$$
d \approx r_{p} \ln \left[\pi \ell_{p} / 4 r_{p}\right]
$$

and the sheath thickness for the two species, $h_{e}$ and $h_{i}$, are [27]

$$
h_{e, i}=0.0013 r_{p}\left(\frac{\lambda_{e, i} \ell_{p} V^{2}}{r_{p}^{2} I_{e, i} \sqrt{T_{e, i} m_{e, i}}}\right)^{0.33} .
$$

Here $\lambda_{e, i}$ is the electron or ion mean free path (defined below), $m_{e, i}$ is the particle mass in units of amu $\left(1 \mathrm{amu}=1.67262 \times 10^{-27} \mathrm{~kg}\right), V$ is the probe potential in volts as before, and $I_{e, i}$ is the electron or ion current, in amps. Note that the sheath thickness changes at different probe voltages, and therefore several analysis regimes may apply to a single $I-V$ characteristic. The appropriate analyses for the ions and electrons may differ as well.

The most important scale lengths to describe the probe system in HELIX are the electron and ion mean free paths, $\lambda_{e}$ and $\lambda_{i}$; the electron energy relaxation length $\lambda_{\varepsilon}\left(\lambda_{\varepsilon, i}=\lambda_{i}\right)$; the Debye length $\lambda_{\mathrm{D}}$ (see Eq. (3.6)); the electron and ion gyro-radii $\rho_{e}$ and $\rho_{i}$; and the charge exchange collision length $\lambda_{\mathrm{cx}}$.

An empirical estimate for the electron and ion mean free paths is [123, p. 28]

$$
\lambda_{e, i} \approx v_{t h ; e, i} / \nu_{e, i}
$$

where $\nu$ is the collision frequency and $v_{t h ; e, i}=\sqrt{2 k_{\mathrm{B}} T_{e, i} / m_{e, i}}$. The collision frequencies are provided by the NRL plasma formulary [123], converted here to SI, with $T_{e, i}$ in $\mathrm{eV}$ :

$$
\begin{gathered}
\nu_{e}=2.91 \times 10^{-12} n_{e} \ln \Lambda T_{e}^{-3 / 2} \\
\nu_{i}=4.80 \times 10^{-14} Z^{4} n_{i}\left(m_{i} / m_{p}\right)^{-1 / 2} \ln \Lambda T_{i}^{-3 / 2}
\end{gathered}
$$

Here, $m_{p}$ is the proton mass: $m_{p}=1.67262 \times 10^{-27} \mathrm{~kg}$, and $\ln \Lambda$ is the Coulomb logarithm, from $\ln (\Lambda)=\ln \left(12 \pi n \lambda_{D}^{3}\right) \approx 10$ [66, p. 181]. Simplifying all the constants, 
and assuming that the argon ions are singly-ionized $(Z=1)$,

$$
\begin{aligned}
& \lambda_{e}=1.45 \times 10^{16} \frac{T_{e}^{2}}{n_{e}} \\
& \lambda_{i}=2.04 \times 10^{16} \frac{T_{i}^{2}}{n_{i}},
\end{aligned}
$$

where $T_{e}$ and $T_{i}$ are in $\mathrm{eV}$, and $n_{e}$ and $n_{i}$ are in $\mathrm{m}^{-3}$, producing $\lambda_{e, i}$ in $\mathrm{m}$. (The constants have units of $\mathrm{m}^{4} \mathrm{eV}^{-2}$.) If $e$-e collisions occur more frequently than collisions with other species, then $\lambda_{\varepsilon} \approx \lambda_{e}$. However, if $e-\operatorname{Ar}^{0}$ collisions dominate, then [27]

$$
\lambda_{\varepsilon} \approx \frac{\lambda_{e}}{\sqrt{2 m_{e} / m_{\mathrm{Ar}}}} \approx 60 \lambda_{e} .
$$

The gyro-radii are

$$
\rho_{e, i}=v_{\perp} / \omega_{c e, i}=\frac{\left(2 T_{e, i} m_{e, i}\right)^{1 / 2}}{e B}
$$

and finally the charge exchange length is

$$
\lambda_{\mathrm{cx}}=\left(n_{n} \sigma_{\mathrm{cx}}\right)^{-1}
$$

The charge exchange cross-section $\sigma_{\mathrm{cx}}$ is obtained from literature [1, 124, 125]. Cross section data is difficult to obtain for low energies, but the data that exists can be extrapolated to lower energies. The data from Ref. [1] fits well to the relation

$$
\sigma_{\mathrm{cx}}\left(10^{-20} \mathrm{~m}^{2}\right) \approx 59.28-3.257 \times 10^{-4} E_{i}-4.641 \ln \left(E_{i}\right)
$$

At $E_{i}=0.35 \mathrm{eV}$, Eq. (3.28) estimates the cross section to be $\sigma_{\mathrm{cx}}=6.4152 \times 10^{-19}$ $\mathrm{m}^{2}$. The ideal gas law provides a crude estimate of the neutral density. At $T_{n}=$ $0.027 \mathrm{eV}, 3.6$ mTorr corresponds to $n_{n} \approx 1.11 \times 10^{20} \mathrm{~m}^{-3}$, for a charge exchange length $\lambda_{\text {cx }} \gtrsim 14 \mathrm{~mm}$. The neutral density decreases once the discharge is struck, so using the pre-discharge pressure gives the most conservative estimate for $\lambda_{\mathrm{cx}}$.

In the presence of a strong magnetic field, Eqs. (3.18)-(3.27) must be modified to reflect the asymmetry in perpendicular and parallel diffusion. Demidov et al. reduce the perpendicular quantities by the parameter $\eta_{e, i}=\left(1+\left(\lambda_{e, i} / \rho_{e, i}\right)^{2}\right)^{1 / 2}$, 


\begin{tabular}{cccc}
\hline \hline$r_{p}$ & $2.5 \times 10^{-4}$ & $r_{h}$ & $5.0 \times 10^{-4}$ \\
$d=d_{\|}$ & $4.6 \times 10^{-4}$ & $d_{i \perp}$ & $4.1 \times 10^{-4}$ \\
$\ell_{p}$ & $2.0 \times 10^{-3}$ & $d_{e \perp}$ & $1.2 \times 10^{-7}$ \\
$h_{e}$ & $5.6 \times 10^{-4}$ & $h_{e \perp}$ & $1.5 \times 10^{-7}$ \\
$h_{i}$ & $1.3 \times 10^{-4}$ & $h_{i \perp}$ & $1.2 \times 10^{-4}$ \\
$\rho_{i}$ & $6.3 \times 10^{-3}$ & $\rho_{e}$ & $8.0 \times 10^{-5}$ \\
$\lambda_{\mathrm{cx}}$ & $1.4 \times 10^{-2}$ & $\lambda_{D}$ & $4.7 \times 10^{-6}$ \\
$\lambda_{i}=\lambda_{i \|}$ & $3 \times 10^{-3}$ & $\lambda_{i \perp}$ & $2.8 \times 10^{-3}$ \\
$\lambda_{e}=\lambda_{e \|}$ & 0.38 & $\lambda_{e \perp}$ & $8.0 \times 10^{-5}$ \\
$\lambda_{\varepsilon}=\lambda_{\varepsilon \|}$ & 18 & $\lambda_{\varepsilon \perp}$ & $1.5 \times 10^{-2}$ \\
\hline \hline
\end{tabular}

TABLE 3.3: Scale lengths in a sample helicon plasma in HELIX, IV\#16 (17 April 2017), where $T_{e}=4.1 \mathrm{eV}, T_{i}=0.35 \mathrm{eV}, n_{e}=n_{i}=n=8 \times 10^{17} \mathrm{~m}^{-3}$, and $B=600$ G. $r_{p}$ - probe radius; $r_{h}$ - radius of the ceramic probe holder; $\ell_{p}-$ probe length; $d$ - characteristic probe dimension; $h_{i}, h_{e}$ - ion and electron sheath thicknesses; $\rho_{i}, \rho_{e}$ - ion and electron gyro-radii; $\lambda_{D}$ - Debye length; $\lambda_{\varepsilon}$ - electron energy relaxation length; $\lambda$ - mean free path. All quantities are in meters.

which describes the relative momentum and gyration scales:

$$
d_{e, i \perp}=d \eta_{e, i}^{-1} \quad \lambda_{e, i \perp}=\lambda_{e, i} \eta_{e, i}^{-1} \quad \lambda_{\varepsilon \perp}=\lambda_{\varepsilon} \eta_{e, i}^{-1}
$$

For the reference characteristic, $B=600 \mathrm{G}, p=3.5 \mathrm{mTorr}, T_{e}=4.1 \mathrm{eV}, T_{i}=0.35$ $\mathrm{eV}, n_{e}=n_{i}=n=8 \times 10^{17} \mathrm{~m}^{-3}$. Conservative approximations for $I_{e, i}$ and $V$ are $I_{e} \approx 0.1 \mathrm{~A},\left|I_{i}\right| \lesssim 1 \times 10^{-3} \mathrm{~A}$, and $|V| \lesssim 50 \mathrm{~V}$. Scale length estimates are shown in Tab. 3.3, along with the alumina electrode holder radius, $r_{h}$.

The magnetic quantities are related via

$$
\lambda_{\varepsilon \perp} \approx \lambda_{\mathrm{cx}}>\rho_{i}>\lambda_{i \perp}>d \approx d_{i \perp}>r_{p}>h_{i \perp}>\rho_{e}=\lambda_{e \perp} \gg \lambda_{D} \gg h_{e \perp} \gtrsim d_{e \perp} .
$$

According to the classification of Ref. [27] for unmagnetized plasmas, this arrangement corresponds to the orbital limited thick sheath analysis regime for the electrons and to the collisionless thin sheath regime for the ions. For calculating the distribution function in the presence of the HELIX magnetic field, the strongly 
magnetized, nonlocal condition $\lambda_{\varepsilon \perp} \gg d \gg \rho_{e}$ is weakly satisfied: $\lambda_{\varepsilon \perp} \approx 33 d \approx 6 \rho_{e}$.

An alternative framework for determining the analysis regime arises from the generalized probed current [94]

$$
I_{e}=C \int_{e U}^{\infty} \frac{(\varepsilon-e U) f_{p}(\varepsilon)}{1+[(\varepsilon-e U) / \varepsilon] \Psi(\varepsilon)} d \varepsilon ; \quad C \doteq \frac{e A_{p}}{\sqrt{8 m_{e}}}
$$

where $U=\phi_{p}-V$. The appropriate analysis hinges on the unitless diffusion parameter $\Psi(\varepsilon)$. When the probe is oriented so that its long axis is perpendicular to the magnetic field, as it is for all of the experiments reported in this document $[27,96]$

$$
\Psi_{\perp}(\varepsilon)=\frac{r_{p}}{\gamma \rho_{e}(\varepsilon)} \ln \left(\pi \ell_{p} / 4 r_{p}\right) .
$$

The unitless geometric factor $\gamma=4 / 3-0.62 \exp \left(-\lambda_{e} / 2 r_{p}\right)$ relates the electron momentum loss scale length $\lambda_{e}$ and the probe radius. $\rho_{e}(\varepsilon)$ is the energy dependent electron gyro-radius. ( $\Psi_{\|}$for probes aligned parallel to the field is given in Refs. $[27,96] . \Psi_{\|}$does not apply to any of the experiments reported in this document, and the $\perp$ designation will be omitted from now on.)

The denominator of Eq. (3.31) establishes three analysis cases:

$\Psi \ll 1$ - Druyvesteyn analysis: $f_{p} \propto I_{e}^{\prime \prime}$ (following Ref. [27]) In the presence of very energetic electrons (low electron collisionality) or very low magnetic fields, the second term in the denominator of Eq. (3.31) is neglected. In the classification of Demidov et al., this case is equivalent to the collisionless regime, when $\rho_{e} \gg r_{p} \ln \left(\ell_{p} / r_{p}\right)$. In this case, the appropriate method, first solved by Druyvesteyn, derives $f_{p}$ from the second derivative of the electron current [126]:

$$
f_{p}(\varepsilon)=\frac{2 \sqrt{2 m_{e}}}{e^{3} A_{p}} \frac{d^{2} I_{e}}{d U^{2}}
$$

$\phi_{p}$ is the voltage at max $I_{e}^{\prime}$ or at the zero-crossing of $I_{e}^{\prime \prime}=0$.

$\Psi \gg 1$ - Strong magnetic field: $f_{p} \propto I_{e}^{\prime}$. When the diffusion parameter is very large the probe predominantly collects electrons tied to the field lines that 
intersect the probe surface. In these circumstances, the EEPF is found not from the second derivative of the current but the first. The form of the EEPF is then calculated to be $[94,127]$

$$
f_{p}(e U)=\frac{3 \sqrt{2 m_{e}}}{2 e^{3} A_{p}} \frac{\gamma \Psi}{U} \frac{d I_{e}}{d U}
$$

This formulation has the additional benefit of requiring one fewer digital derivative and is therefore less prone to noise amplification. In the classification of Demidov et al., this case is equivalent to the strongly magnetized, nearly nonlocal regime $\lambda_{\varepsilon \perp} \gg d \gg \rho_{e}[27]$.

$\Psi \sim 1$ - Extended Druyvesteyn analysis. When neither limit applies, the extended current relation [94]

$$
I_{e}^{\prime \prime}(U)=C f_{p}(e U)-C \int_{e U}^{\infty} K^{\prime \prime}(W, U) f_{p}(W) d W
$$

should be solved, where

$$
K^{\prime \prime}(W, U)=\frac{2 \Psi W^{2}}{[W(1+\Psi)-\Psi e U]^{3}}, \quad C \doteq \frac{e^{3} A_{p}}{2 \gamma \sqrt{2 m_{e}}}
$$

$W=m_{e} v_{s h}^{2} / 2+e U$, and $v_{s h}=\sqrt{8 \varepsilon / \pi m_{e}}$ is the electron velocity at the sheath edge. In the absence of the second term of (3.35), when $\Psi \ll 1$, $I_{e}^{\prime \prime}(U)=C f(e U)$ returns the conventional Druyvesteyn formula in Eq. (3.33). The second term corrects for the anisotropic diffusion geometry which causes electrons along field lines intersecting the probe surface to be preferentially sampled. (Note that Refs. [94] and [127] present Eq. (3.35) in terms of the EDF; $C$ reflects this difference.) The disadvantage of this method is that it is complicated to solve Eq. (3.35) without a priori knowledge of the shape of $f_{p}$ and complicated further by a shift that occurs in $\phi_{p}$ up to $1.4 T_{e}$ from the $I_{e}^{\prime \prime}\left(\phi_{p}\right)=0$ potential estimate that is typically relied on [94]. If the shape of the distribution can be determined from another method, a model distribution can be constructed that fits to $I_{e}^{\prime \prime}$, with the plasma quantities determined as fit parameters. 


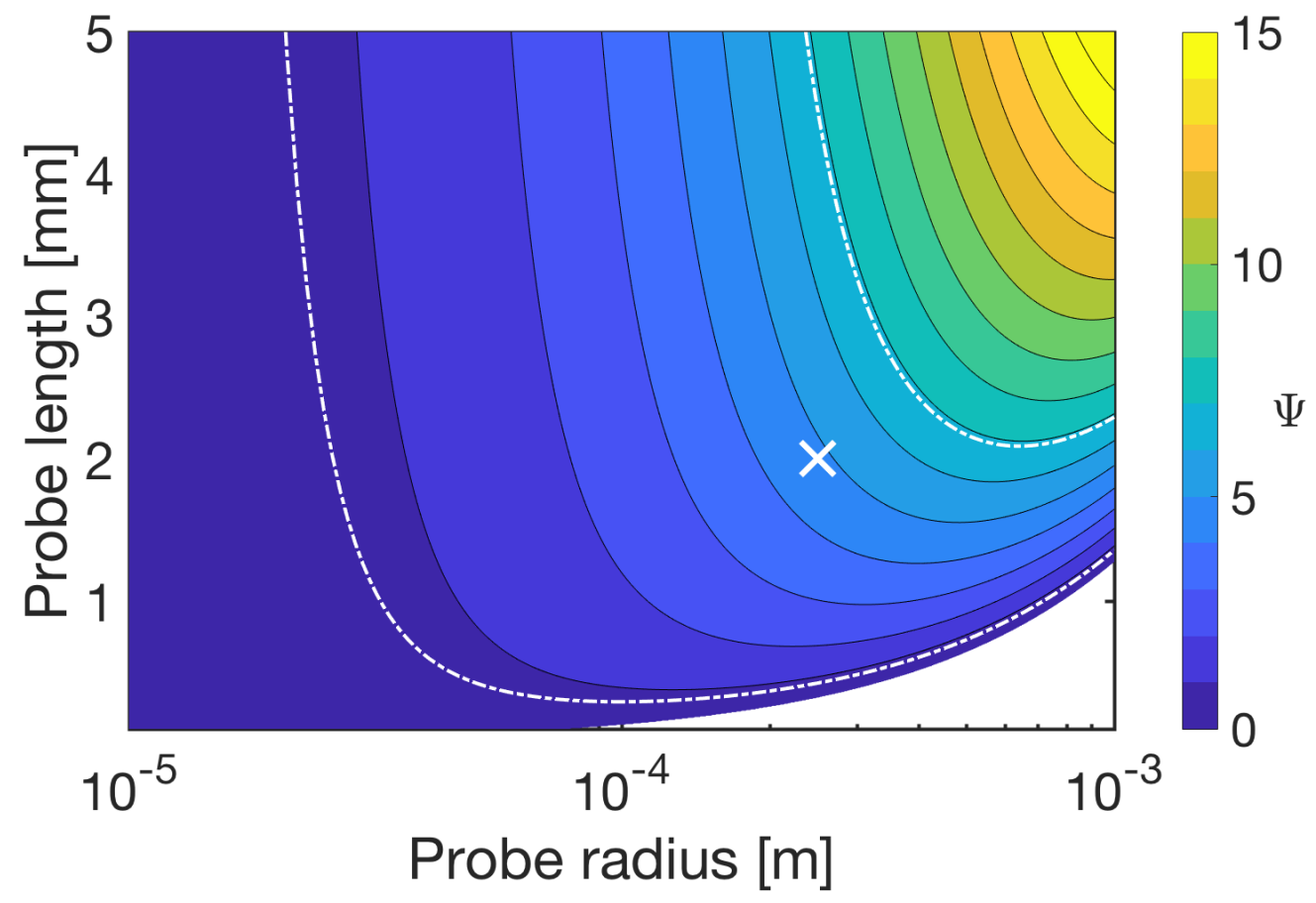

Figure 3.10: $\Psi$ range for $T_{e}=4 \mathrm{eV}$ and $B=600$ gauss. The white $\mathrm{X}$ marks $\Psi$ when $r_{p}=250 \mu \mathrm{m}$ and $\ell_{p}=2 \mathrm{~mm}$ for typical HELIX plasma conditions. The white dashed lines indicate $\Psi=0.75$ and 7 .

There is some disagreement on how large $\Psi$ must be for the $\Psi \gg 1$ limit to apply, with some authors requiring $\Psi>7$ [96] and others as much as $\Psi>100$ [128]. Fig. 3.10 shows the range of $\Psi$ available for the plasma conditions of the experiments reported here. A white ' $\mathrm{X}$ ' marks $\Psi$ for the dimensions of the specific Langmuir probe described in Tab. 3.3. Fig. 3.11 shows the range of $\Psi$ accessible by HELIX magnetic fields for these probe dimensions. White dashed lines indicate $\Psi=0.75$ and 7 .

For the majority of plasmas generated in HELIX, $\Psi$ falls in the third category: $\Psi \sim 1$. At $B=600 \mathrm{G}$ and for typical temperatures, $\Psi>7$ for $\varepsilon \lesssim 3.5 \mathrm{eV}$ and barely dips below 0.75 at $100 \mathrm{eV}$. In fact, under these plasma conditions $\Psi$ cannot be brought below unity for the majority of the electron energies except for probe diameters of tens of microns. The most appropriate analysis therefore follows extended Druyvesteyn theory. The procedure is to calculate $f_{p}$ in the strongly magnetized field limit, compare it against the conventional Druyvesteyn EEPF, and then attempt to reconstruct $I_{e}^{\prime \prime}$ with a model $f_{p}$ whose form is determined by the EEPFs calculated by the first two methods. 


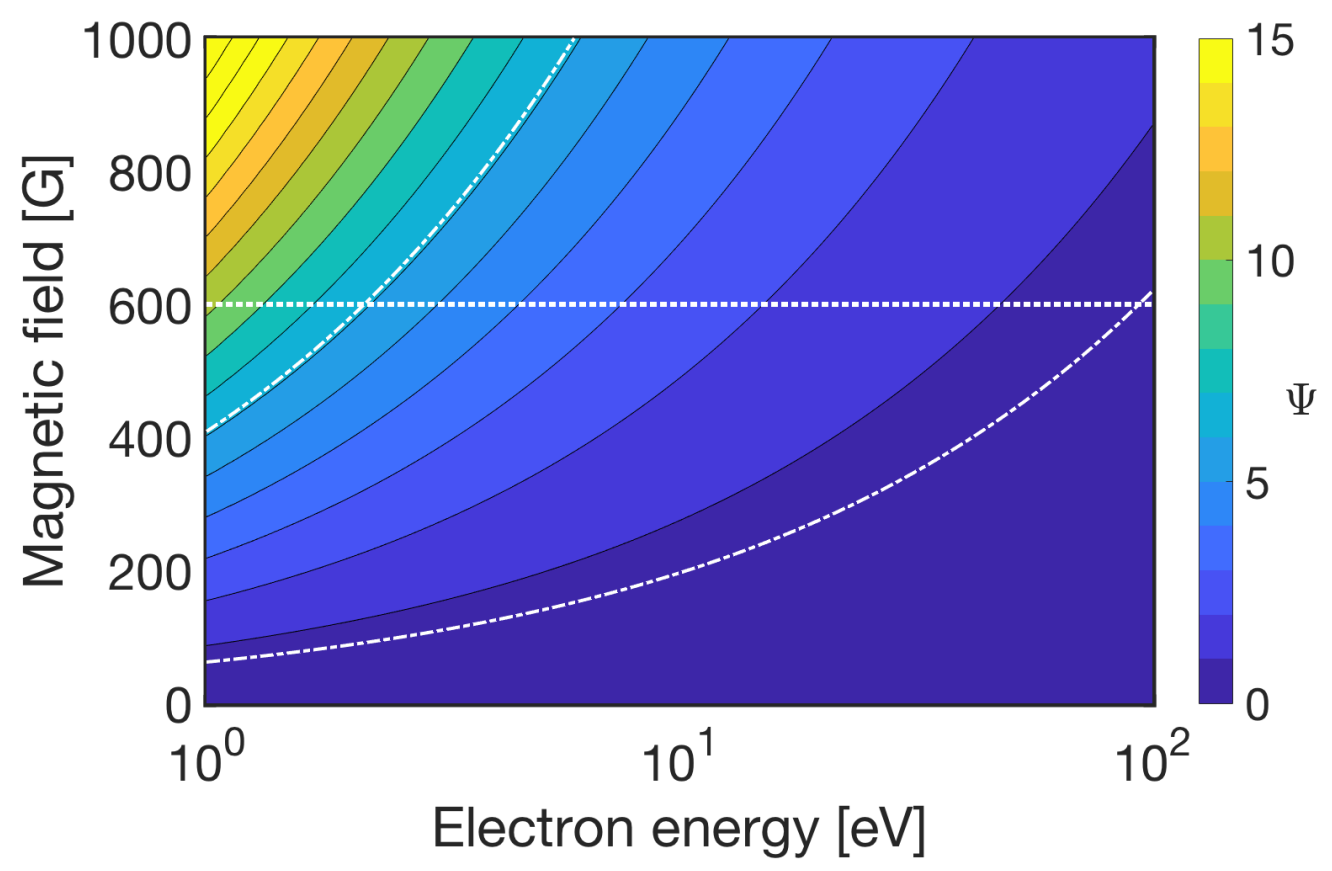

FiguRE 3.11: $\Psi$ over the range of HELIX plasmas for $r_{p}=250 \mu \mathrm{m}$ and $\ell_{p}=2$ $\mathrm{mm}$. The white dashed lines indicate $\Psi=0.75$ and 7 .

\section{Druyvesteyn analysis}

An example EEPF calculated from characteristic IV\#16 using conventional Druyvesteyn analysis described by Eq. (3.33) is shown in Fig. 3.12. The EEPF is Maxwellian over two decades. The apparent low energy depletion, i.e., the rounded peak, is an artifact of the probe's finite energy resolution. A linear extrapolation accounts for this distortion and is used to calculate $n$ and $T_{\text {eff }}$ when the EEPF is Maxwellian.

\section{Strongly magnetized, nonlocal analysis}

An example EEPF calculated by the strongly magnetized nearly nonlocal analysis of Eq. (3.34) is shown in Fig. 3.13. The low energy portion of the EEPF is distorted by the division by $U \sim 0$, and a linear fit to this Maxwellian distribution

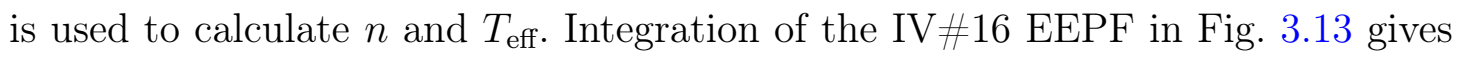
$n=1.95 \times 10^{18} \mathrm{~m}^{-3}$ and $T_{\text {eff }}=2.8 \mathrm{eV}$. Recall that the strongly magnetized regime is marginally valid for this test $I-V$ characteristic. The analysis assumes that the probe undersamples the bulk electron population because it overestimates the 


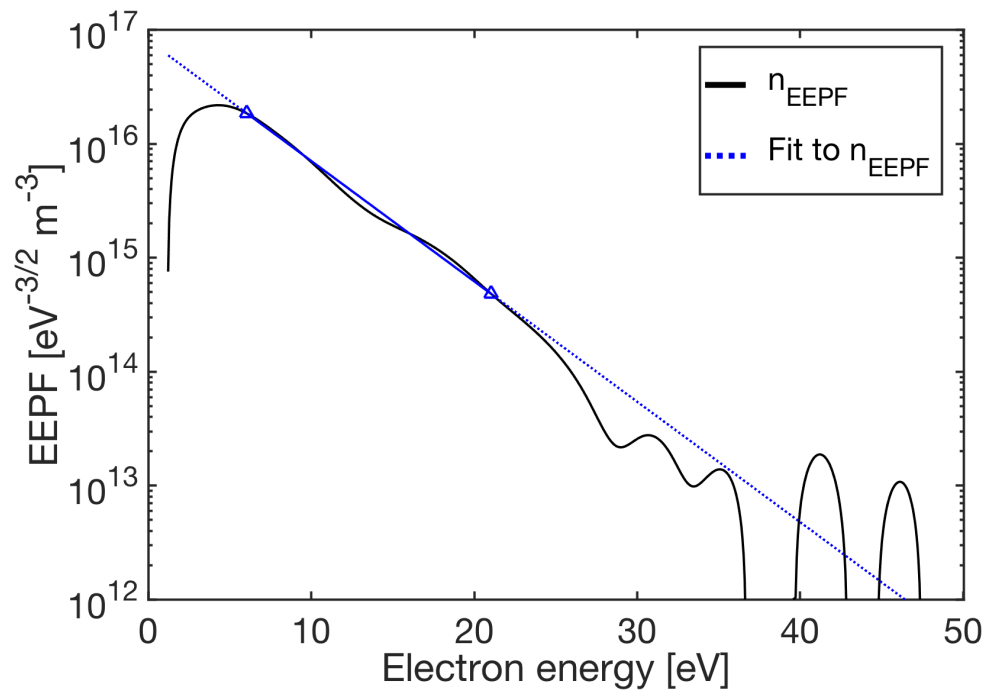

Figure 3.12: Example EEPF calculated from IV\#16 using conventional Druyvesteyn analysis, with extrapolation to low energies.

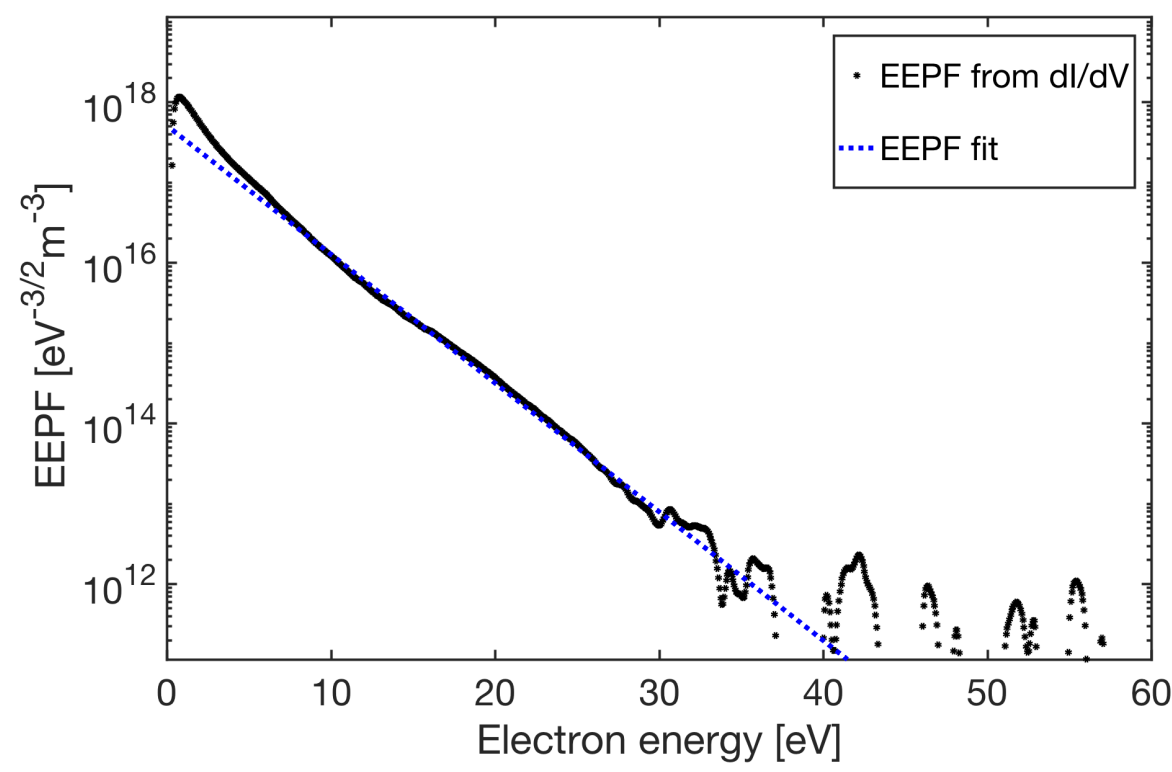

Figure 3.13: Example EEPF calculated from IV\#16 (17 April 2017) using strongly magnetized, nearly nonlocal analysis. $n=1.95 \times 10^{18} \mathrm{~m}^{-3}, T_{\text {eff }}=2.8$ $\mathrm{eV}$.

magnetic confinement of the electrons along field lines. The result is an overestimation of the unperturbed density. $T_{\text {eff }} \propto n^{-1}$, and therefore the reported temperature is low. 


\section{Extended Druyvesteyn analysis}

An example extended Druyvesteyn analysis using Eq. (3.35) is shown in Fig. 3.14. Following Ref. [94], a model of $I_{e}^{\prime \prime}$ is created using an assumed Maxwellian or bi-Maxwellian EEPF:

$$
\begin{aligned}
\text { Max.: } \quad f_{p}^{\text {model }} & =\frac{2 n}{\sqrt{\pi}} T_{e}^{-3 / 2} e^{-\varepsilon / T_{e}} \\
\text { Bi-Max.: } \quad f_{p}^{\text {model }} & =\frac{2 n_{e, \mathrm{~B}}}{\sqrt{\pi}} T_{e, \mathrm{~B}}^{-3 / 2} e^{-\varepsilon / T_{e, \mathrm{~B}}}+\frac{2 n_{e, \mathrm{E}}}{\sqrt{\pi}} T_{e, \mathrm{E}}^{-3 / 2} e^{-\varepsilon / T_{e, \mathrm{E}}}
\end{aligned}
$$

Here subscript $B$ and $E$ mark the bulk and energetic populations. As shown in Fig. 3.15, the correction term falls off sharply at high energies; the correction acts primarily on the low energies. For this reason, $T_{\text {eff }}$ is determined by optimizing the fit in the high energy region. Matching at low energies provides $\phi_{p}$. With these parameters set, $n$ is determined by scaling the model and matching it to the data in a $\chi^{2}$-minimized sense. The uncorrected EEPF appears as a straight line when using a Maxwellian model and matches the adjusted EEPF at high energies. An important feature of this correction is that the plasma potential designated at the maximum of $I_{e}^{\prime}$ (or equivalently at $I_{e}^{\prime \prime}=0$ ) occurs approximately $1 T_{\text {eff }}$ less than the actual $\phi_{p}$, marked by the vertical line in Fig. 3.14. Without correction, this inaccuracy in $\phi_{p}$ leads to additional inaccuracies in calculations of $n$ and $T_{\text {eff }}$ calculated by taking moments of $f_{p}$.

\section{Comparison of the results from different techniques}

The techniques in this section are applied to each $I-V$ characteristic recorded near the boundary. The results from the test characteristic IV\#16 (17 April 2017) are contained in Tab. 3.4. This characteristic is the first non-calibration Langmuir probe data recorded during the campaign to measure the plasma in the vicinity of an absorbing boundary in HELIX. The various methods show a range similar to the variation observed by other groups (see, for example, Ref. [129]). The range of densities, including both ions and electrons, is $4.3-19.5 \times 10^{17} \mathrm{~m}^{-3}$, with the 


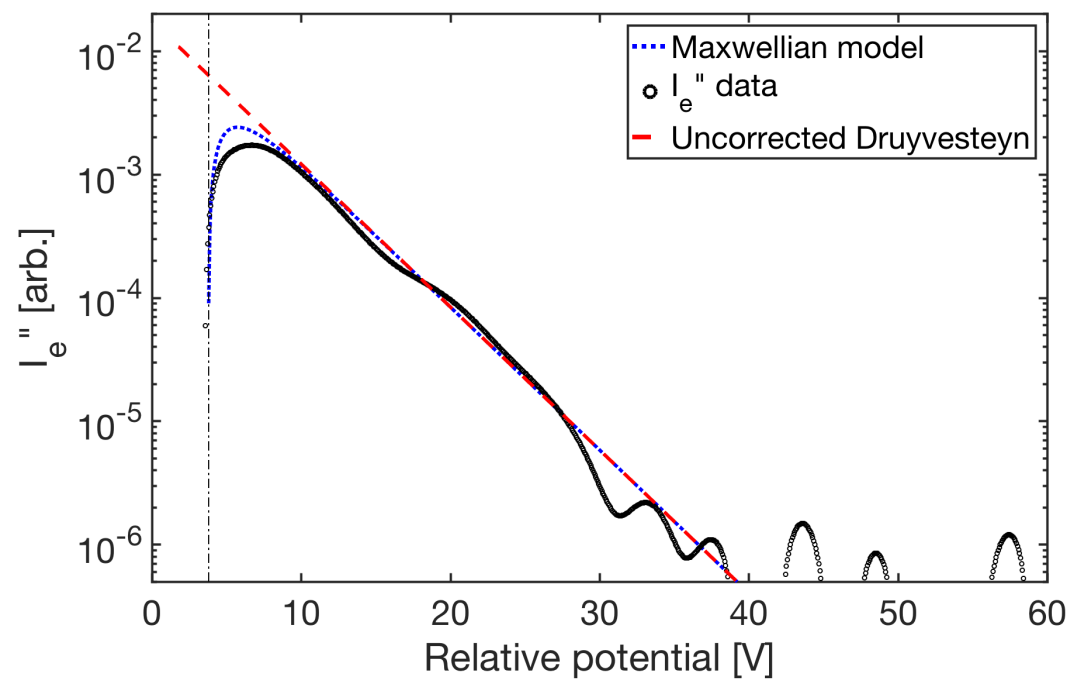

Figure 3.14: Second derivative reconstructed using extended Druyvesteyn analysis and compared to uncorrected Druyvesteyn current. These data show that $T_{\text {eff }}=3.8 \mathrm{eV}, n_{e}=7.5 \times 10^{17} \mathrm{~m}^{-3}$ and $\phi_{p}=17.19 \mathrm{~V}$, as indicated by the vertical dashed line. The $I_{\phi}^{\prime \prime}=0$ potential occurs here at $U=0$.

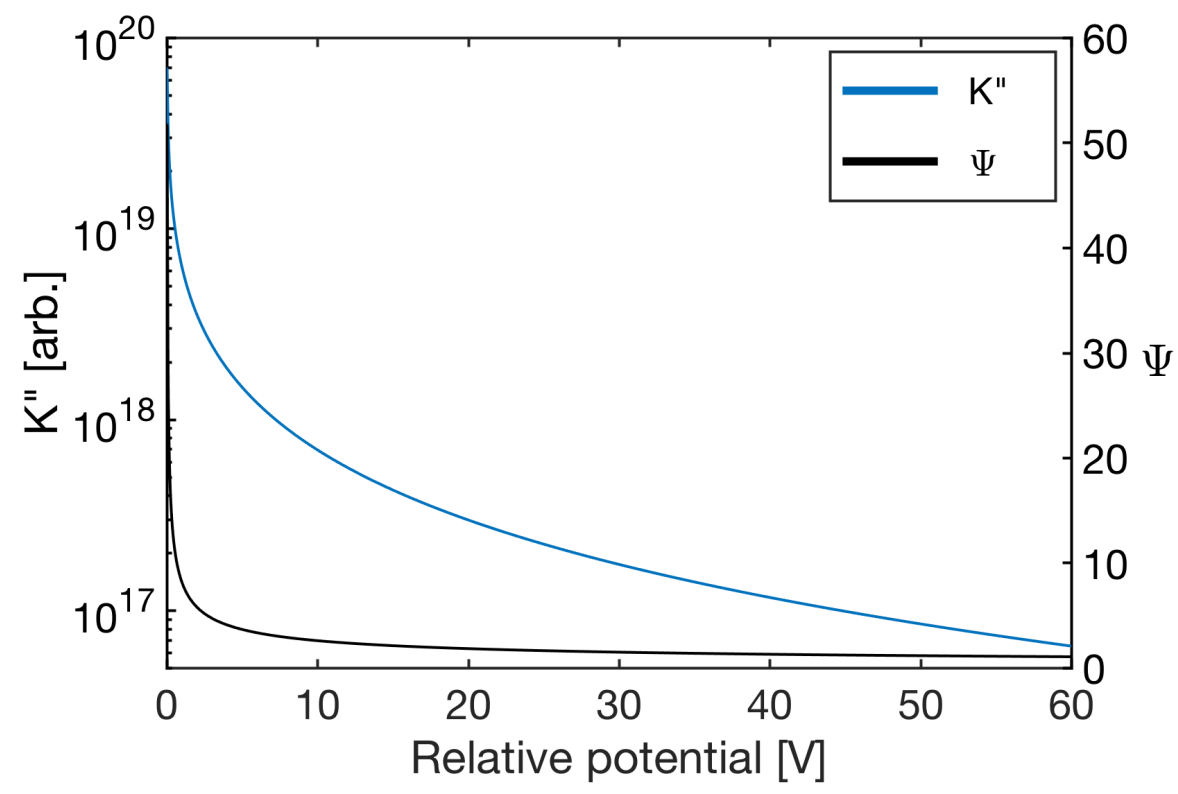

FiguRE 3.15: Dependence of $K^{\prime \prime}$ and $\Psi$ on relative potential.

minimum and maximum a factor of 4.5 apart. The spread in temperatures is high, from 2.79 to $4.13 \mathrm{eV}$, presumably due to the inclusion of several invalid analysis techniques. The $2.79 \mathrm{eV}$ temperature derived from the strongly magnetic analysis is an outlier, and its disparity is easily traced back to an overestimation of plasma density. The plasma potential determined at the knee and at the zero-crossing of the second derivative agree well. However, extended Druyvesteyn analysis indicates 


\begin{tabular}{|c|c|c|c|c|}
\hline Technique & $n[10$ & $\left.7 \mathrm{~m}^{-3}\right]$ & $T_{e}$ or $T_{\text {eff }}[\mathrm{eV}]$ & $\phi_{p}[\mathrm{~V}]$ \\
\hline Conventional Langmuir & $\begin{array}{l}19.2 \\
13.0\end{array}$ & $\begin{array}{l}\text { (electrons) } \\
\text { (ions) }\end{array}$ & 3.97 & 15.96 \\
\hline Orbital Motion Limited & 4.3 & (ions) & 3.44 & 16.04 \\
\hline Child-Langmuir floating point & 17.7 & (ions) & 3.98 & $15.76^{\dagger}$ \\
\hline Druyvesteyn (fit), from $I_{e}^{\prime \prime}$ & 5.9 & & 4.13 & $15.76^{\dagger}$ \\
\hline Strongly magnetized, from $I_{e}^{\prime}$ & 19.5 & & 2.79 & $15.76^{\dagger}$ \\
\hline Extended Druyvesteyn & 7.5 & & 3.8 & 17.19 \\
\hline
\end{tabular}

TABLE 3.4: Summary of the results of fluid quantities for characteristic IV\#16 (17 April 2017) calculated using various probe analyses. $\dagger$ - Derived from $\max \left(I_{e}^{\prime}\right)$ or $I_{e}^{\prime \prime}=0$.

that these results may be $\approx 0.3 T_{\text {eff }}$ too low, and that the correct plasma potential is $17.2 \mathrm{~V}$.

The conventional Langmuir analysis produces an electron temperature and plasma potential in agreement with the temperatures derived from other methods. The electron density is suspect because it is difficult to precisely identify the electron saturation onset near the current limit of the circuit. The ion measurement is more credible because it is not at risk of distortion from current limitations and because the scaling analysis places the ions firmly in the collisionless thin sheath analysis regime, even in the HELIX magnetic field.

The OML method produces a temperature which is lower than most of the other methods, but not unreasonably so. This can be explained by the over-subtraction of the ion contribution which preferentially depletes the energetic region of the electron current and lowers the calculated temperature. The density from OML is lower than the density derived from the other methods. The low density is a consequence of the poorly fit ion region and the disparity ( $\approx 65 \mathrm{~V}$ !) between the plasma potential and the stand-in $V_{p}^{\mathrm{OML}}$ determined from the linear fit intercept. 
OML theory requires no acceleration region and therefore applies only when $\xi_{p}=$ $r_{p} / \lambda_{D} \sim 1$ [113] - well below $\xi_{p} \approx 50$ in these measurements. Sanmartín and Estes have calculated the maximum radius below which OML is valid. For probe bias $|V|<100 \mathrm{~V}, T_{i} \approx 0.4 \mathrm{eV}$, and $T_{e} \approx 4 \mathrm{eV}, \xi_{p}^{\max } \ll 1$ and decreases as the temperature ratio increases [118]. In addition, in the test characteristic the ion current dependence increases gradually with $V$, as $I^{1 / 3}$, instead of $I^{2}$ as predicted by OML theory. These factors suggest that the OML quantities should be considered corroborative or exploratory, but the results from OML should not be considered the preferred results for this characteristic.

The Child-Langmuir floating point density produces the highest density determined from the ion region of the characteristic. The ion current as $I_{i}^{4 / 3}$ appears to match a linear fit better than a linear fit to $I_{i}^{2}$. However, this dependence is still substantially above the $I^{1 / 3}-V$ dependence of the data. Nonetheless, the consistency of this method has been shown to be excellent [115], although published data are limited for $\xi_{p}>9$.

Unlike the three methods mentioned above, the EEPF methods make no a priori assumptions about the shape of the electron energy distribution, which makes them attractive techniques for calculating the fluid quantities. Of the analyses derived from the EEPF, the conventional Druyvesteyn analysis wherein $f_{p}(\varepsilon) \propto I_{e}^{\prime \prime}$ has the weakest theoretical support for use in these plasma conditions. The integral term in Eq. (3.35) suggests that, due to the undersampling of the electrons in the presence of strong fields, the density in the unperturbed region is higher than the result of the conventional Druyvesteyn calculation, so it is not surprising that the conventional analysis reports a low density and consequently the highest temperature. In a similar way, the strongly magnetized analysis overcorrects for the undersampling, resulting in densities that are high and temperatures that are suspiciously low. As expected, the extended Druyvesteyn treatment produces results between these two extremes. The temperature for this Maxwellian distribution is $3.8 \mathrm{eV}$, near the temperatures produced by the other analyses and not affected by an outlier density result. This analysis also indicates a correction of about $1-1.5$ $\mathrm{V}$ for $\phi_{p}$, compared to the other methods. The extended Druyvesteyn analysis is 
the most general and theoretically sound treatment for the probe data and should be preferred whenever it is possible to perform.

\subsubsection{Other considerations}

\section{Energy resolution}

Langmuir probes have finite energy resolution, depending on the design of the probe and its circuit. If carefully designed, the energy resolution is a fraction of the electron temperature, resulting in an accurate measure of the EEPF. Finite energy resolution predominately affects the near-zero region of the EEPF, where the function undergoes the most rapid change, leading to a rolled peak and inaccurate calculations of the distribution moments: $n$ and $T_{\text {eff. }}$ A posteriori, one can measure the separation between the zero-crossing of the second derivative and the neighboring $I_{e}^{\prime \prime}$ maximum, which for acceptable energy resolution should satisfy the condition

$$
\left.\Delta \varepsilon \approx \varepsilon\right|_{\max \left(I_{e}^{\prime \prime}\right)}-\left.\varepsilon\right|_{I_{e}^{\prime \prime}=0} \leq(0.3-0.7) T_{e}
$$

Exceptional energy resolution requires $\Delta \varepsilon \leq 0.3 T_{e}$. For $\Delta \varepsilon>0.7 T_{e}$, best practice is to fit the low energy region of the EEPF and extrapolate to $\varepsilon=0$, then use the extrapolated function when calculating $n$ and $T_{\text {eff }}[96,106]$. The function [130]

$$
g(\varepsilon)=a \exp \left(-b \varepsilon^{x}\right)
$$

captures both Maxwellian $(x=1)$ and Druyvesteyn $(x=2)$ distribution shapes, with $a, b$ and $x$ set by the fit. The $I_{e}^{\prime \prime}$-derived EEPFs of IV \#16 in Figs. 3.123.14 fit Eq. (3.37) with $x=1.02\left(R^{2}=0.9998\right.$; RMSE $\left.=7.0 \times 10^{-5}\right)$. A biMaxwellian function $g=g_{1}+g_{2}$ gives $x_{1}=0.99$ and $x_{2}=0.17\left(R^{2}=0.9998\right.$; RMSE $=7.4 \times 10^{-5}$ ), which is essentially a single Maxwellian plus a constant, suggesting that the data in characteristic IV \#16 are best described by a single Maxwellian distribution. 


\section{Probe-plasma interactions}

Plasma perturbation caused by the introduction of an electrostatic probe is a well-known problem in plasma diagnostics. The high conductivity and therefore boundary-sensitive nature of plasmas takes on practical importance when dealing with probes. Two classes of interaction must be mitigated for accurate measurements from probe diagnostics: probe perturbation of the background plasma and plasma distortion of probe characteristics.

Probes unavoidably change the plasma equilibrium by absorbing or injecting particles and energy into the plasma during operation [131]. Probes introduce foreign materials into the plasma, which mix with the plasma as impurities, particularly when sputtering and ablation occur. In the most severe cases, energetic electrons streaming toward or away from the probe, or created by secondary impact from energetic ions, can create a plasma-within-a-plasma, which the probe then measures. In general, the probe collecting surface, compensation electrode, and shielding all rearrange the local potential structure in their vicinity. An early warning sign that the validity of probe data is suspect occurs when the plasma visibly changes, shakes, or moves during the acquisition of a probe characteristic. If this phenomenon cannot be eliminated, the probe may have to be redesigned to avoid such disturbances.

As a guiding principle, probe electrodes and their holders should be small. An underlying assumption to many probe analyses is that the potential across the chamber wall sheath is negligibly small. Godyak and Demidov state the requirement as [96]

$$
\frac{A_{p} N_{o}}{A_{\mathrm{ch}} N_{\mathrm{s}}}\left(\frac{m_{i}}{2 \pi m_{e}}\right)^{1 / 2} \ll 1
$$

for Maxwellian electrons, or

$$
\frac{A_{p} N_{o}}{A_{\mathrm{ch}} N_{\mathrm{s}}}\left(\frac{m_{i}}{2 \pi m_{e}}\right)^{1 / 2} \ll\left(\frac{T_{\mathrm{eb}}}{T_{\mathrm{eh}}}\right)^{1 / 2}<1
$$

for bi-Maxwellian distributions. $A_{p}=2 \pi r_{p} \ell_{p}$ is the surface area of the cylindrical probe; $A_{\mathrm{ch}}$ is the collection area of the chamber wall; $N_{o} / N_{\mathrm{s}}$ is the ratio of 


\begin{tabular}{cccc}
\hline \hline$N_{o}$ & $5 \times 10^{17} \mathrm{~m}^{-3}$ & $N_{\mathrm{s}}$ & $\approx N_{o} / 100$ \\
$A_{p}$ & $3.3 \mathrm{~mm}^{2}$ & $m_{\mathrm{Ar}}$ & $6.6 \times 10^{-27} \mathrm{~kg}$ \\
$r_{\mathrm{ch}}$ & $7.5 \mathrm{~cm}$ & $m_{\mathrm{e}}$ & $9.1 \times 10^{-31} \mathrm{~kg}$ \\
$\ell_{\mathrm{ch}}$ & $63 \mathrm{~cm}$ & $T_{\mathrm{eb}}$ & $3.5 \mathrm{eV}$ \\
$A_{\mathrm{ch}}$ & $0.3 \mathrm{~m}^{2}$ & $T_{\mathrm{eh}}$ & $7.5 \mathrm{eV}$ \\
\hline \hline
\end{tabular}

TABLE 3.5: HELIX chamber dimensions and typical plasma conditions.

the density of the plasma in the core of the discharge to the density at the edge; $T_{\mathrm{eb}}$ and $T_{\mathrm{eh}}$ are the bulk and hot electron population temperatures, respectively. Representative quantities for the HELIX chamber and helicon plasmas used for boundary experiments are shown in Tab. 3.5. (Note that several of these quantities were determined from Langmuir probe analysis and are not independently confirmed by other means.) LEIA and the other absorbing surfaces that are not part of the stainless steel section of HELIX are excluded to be conservative. The left-hand side of Eqs. (3.38) and (3.39) for the quantities in Tab. 3.5 is 0.04. For a typical bi-Maxwellian electron distribution in these plasmas, $\left(T_{\mathrm{eb}} / T_{\mathrm{eh}}\right)^{1 / 2} \approx 0.68$. For either distribution, these probe dimensions easily satisfy the check and the probe stands a good chance of measuring the plasma without an outsized effect on the plasma equilibrium.

\section{End effects}

Short probes improve spatial resolution but can introduce unintended end effects. For example, ions approaching the probe build up charge near the electrode. This space charge concentrates near the insulator end of the electrode, creating an electric field directed away from the insulator. This field distorts the symmetry of the potential structure around the electrode. The end effect loss current has been calculated in Ref. [120]:

$$
\frac{I_{\mathrm{loss}}}{I_{i}}=\frac{1}{\sqrt{2}} \frac{r_{p}}{\ell_{p}}\left(\frac{\left(\alpha^{\prime}\right)^{2}}{\alpha_{o}^{2}}-1\right)
$$


where $\alpha^{\prime}$ is given by Eq. (3.5) and $\alpha_{o}=\exp (-1 / 2)$, as usual. For the probe geometry in Tab. 3.3, $I_{\text {loss }} / I_{i} \approx 2.7 \%$. This level of loss is well below the acceptable uncertainty of the measurement and is neglected for this characteristic. Hoskinson and Hershkowitz [132] demonstrated that end effects influence the characteristic for a broad range of plasma conditions and $r_{p} / \lambda_{D}$, and therefore the ion loss ratio is monitored for every characteristic in case they require correction.

\subsubsection{Electrostatic fluctuations}

If electrostatic fluctuations are present in the plasma, measurements of these fluctuations provide frequency-space insights for determining the underlying physical processes near the boundary. These measurements are conducted using a probe without RF compensation. Instead of connecting the probe BNC output to the Keithley SourceMeter, the output is connected to a $1 \mathrm{M} \Omega$ AC-coupled channel on the oscilloscope. At full bandwidth, a $500 \mathrm{MS} / \mathrm{s}$ or $1 \mathrm{GS} / \mathrm{s}$ timeseries is recorded.

The power spectral density (PSD) of the probe data describes how the signal power, or square of the signal voltage, is distributed in frequency space. In other words, the PSD describes at what frequencies fluctuations in the plasma occur [133, p. 118]. An estimate of the PSD is provided by the Matlab function periodogram() [134]. For a timeseries signal $S_{n}(t)$, the periodgram (PSD estimate) is

$$
\hat{P}(f)=\frac{\Delta t}{N}\left|\sum_{n=0}^{N-1} S_{n} e^{-i 2 \pi f n}\right|^{2}, \quad-\frac{1}{2 \Delta t}<f \leq \frac{1}{2 \Delta t}
$$

from the function call

$$
[\mathrm{Pxx}, \mathrm{F}]=\operatorname{periodogram}\left(\mathrm{S}_{\mathrm{n}},[], \text { length }\left(\mathrm{S}_{\mathrm{n}}\right), \mathrm{Fs}\right)
$$

where $\mathrm{Fs}$ is the sample rate in $\mathrm{Hz}$, such that $\Delta t=1 / \mathrm{Fs}$, and the second argument indicates that the PSD estimate Pxx should be calculated using a rectangular window. Fig. 3.16 contains an example PSD estimate recorded at boundary experiment conditions. The RF frequency, $9.5 \mathrm{MHz}$, its half-harmonic, and its first 


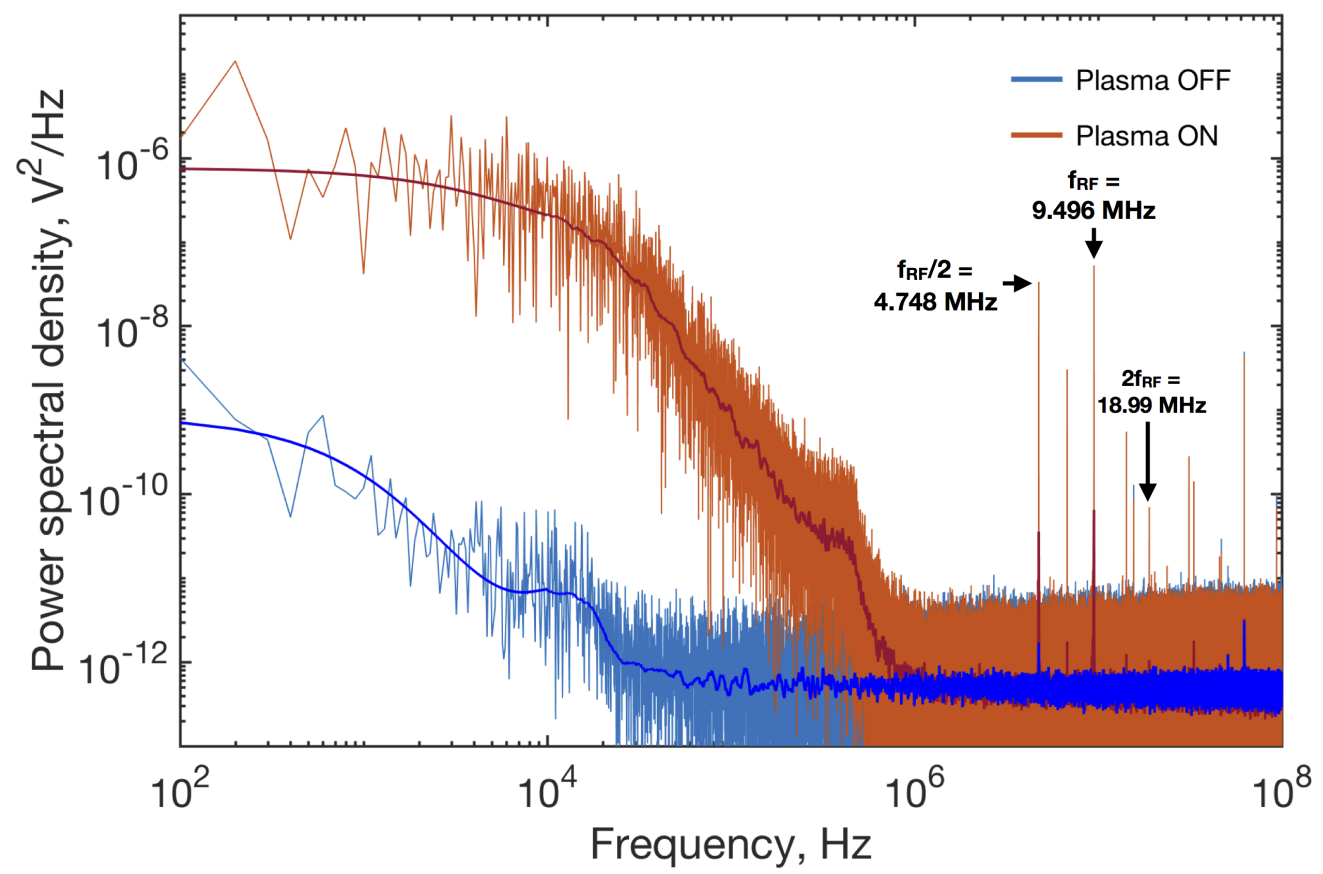

FiguRE 3.16: Power spectral density estimate of floating potential fluctuations at boundary experiment conditions. No RF compensation is present in the circuit. A PSD calculated from "signal" taken in the absence of plasma, but with all other systems running, is included for comparison. The RF frequency, half-harmonic, and first harmonic frequencies are marked.

harmonic are clearly visible when the plasma is on. Only the half-harmonic is visible, at low power, when there is no plasma in the chamber. This is likely because the standby power of the RF power supplies is not zero, but very small, typically $\approx 10 \mathrm{~W}$. To reiterate, these measurements are taken in the absence of any RF compensation. The features above $1 \mathrm{MHz}$ are highly attenuated during Langmuir probe measurements, during which RF compensation is in place.

\subsection{Laser induced fluorescence}

\subsubsection{Overview}

In the vicinity of plasma boundaries, potential structures influence the positive ions more significantly than they influence electrons, which pass rapidly through these structures with minimal acceleration before reaching the boundary surface. Neutral and ion velocity distributions couple through ionization, recombination, 
or charge exchange. Fortunately, boundary structures can be arranged in which the potential and ion-neutral interaction scales are large enough that the velocity distribution variations of the two species are resolvable.

While it is possible to observe the ions - but not the neutrals — via retarding field energy analyzers (RFEAs) or Mach probes, both of these diagnostics are highly perturbative, making accurate analysis of the data in the complicated environment of a magnetized boundary prohibitively difficult. Fortunately, laserinduced fluorescence (LIF) is non-perturbative, and the theory for analyzing LIF data is well established. LIF has been applied to a broad range of applications, from single-photon LIF first introduced by Stern and Johnson in 1975 [135], and two-photon LIF (TALIF) [136, 137], to planar LIF [138, 139], multiplexed LIF [140], tomographic LIF [141], and multispecies LIF using diode lasers [142, 143].

In LIF, a narrow bandwidth laser passes through the sample region of the plasma as its wavelength sweeps through an absorption resonance. After absorption, the target species relaxes to a third state, emitting a photon of known wavelength. The intensity of this emission is associated with the injection wavelength, generating a spectrum for energies around the resonance. The resonance wavelength is defined within the reference frame of the absorbing particles and is therefore Doppler shifted for particles in motion. For this reason, the spectrum provides a distribution of the velocities of the particles within the sample region. This distribution is referred to as a velocity distribution function (VDF). The velocity moments of the VDF provide the bulk flow velocity ( $1^{\text {st }}$ moment $)$ and temperature ( $2^{\text {nd }}$ moment $)$ of the population in the direction of the laser injection. In certain plasma species, the density ( $0^{\text {th }}$ moment $)$ is also obtainable. For argon, however, the absolute density is difficult to obtain without the incorporation of a collisional-radiative model to determine the relative population of states [143]. 


\subsubsection{Velocity distributions from LIF data}

A model of the LIF signal is described in Refs. [110], [144], and [145]:

$$
I_{\mathrm{LIF}}(\nu)=\alpha_{\mathrm{DC}}+I_{\mathrm{LIF}}\left(\nu_{o}\right) \exp \left[-\gamma\left(\nu-\nu_{o}-\left(u_{\mathrm{n}} / c\right) \nu_{o}-\delta \nu_{Z}\right)^{2}\right]
$$

where $\alpha_{\mathrm{DC}}$ accounts for any electronic DC offset, $I_{\mathrm{LIF}}$ is the LIF signal at laser frequency $\nu, \delta \nu_{Z}$ is the Zeeman shift, $\nu_{o}$ is the resonant transition frequency for the particle at rest, $\nu_{o}(u / c)$ is the Doppler shift due to the mean flow $u$, and $\gamma=m_{\mathrm{n}} c^{2} / 2 k_{\mathrm{B}} T_{\mathrm{n}} \nu_{o}^{2}$ is a scaling factor that relates the temperature $T$ of the particle to its mass. For polarized LIF perpendicular to the magnetic field, the Zeeman shift is $\delta \nu_{Z} \sim 0$, and the normalized signal (marked with a $*$ superscript) can be rewritten for velocity dependence:

$$
I_{\mathrm{LIF}}^{*}(v)=\alpha_{\mathrm{DC}}+\exp \left[\frac{-\left(m / 2 k_{B}\right)(v-u)^{2}}{T}\right]
$$

The mean flow $u$ and temperature $T$ are determined directly from the best fit of Eq. (3.44) to the data. For parallel injection, the Zeeman shift term must be included, after which the analysis proceeds in the same way. Bi-Maxwellian distributions are described by adding an additional exponential term to Eq. (3.44).

An example IVDF is shown in Fig. 3.17. The relationship between the temperature and the distribution width is demonstrated in the relative widths of the argon and iodine distributions: the room temperature iodine (black dots) is visibly narrower than the $\sim 10 \mathrm{x}$ hotter ion population. The narrowness of the iodine spectrum also reflects the greater iodine mass: $m_{\mathrm{I}}=3.1768 m_{\mathrm{Ar}}$. The peak of the ion distribution is not noticeably displaced from $0 \mathrm{~m} / \mathrm{s}$, indicating that this population, recorded at the center of the plasma, is not flowing on average in the direction of laser injection. 


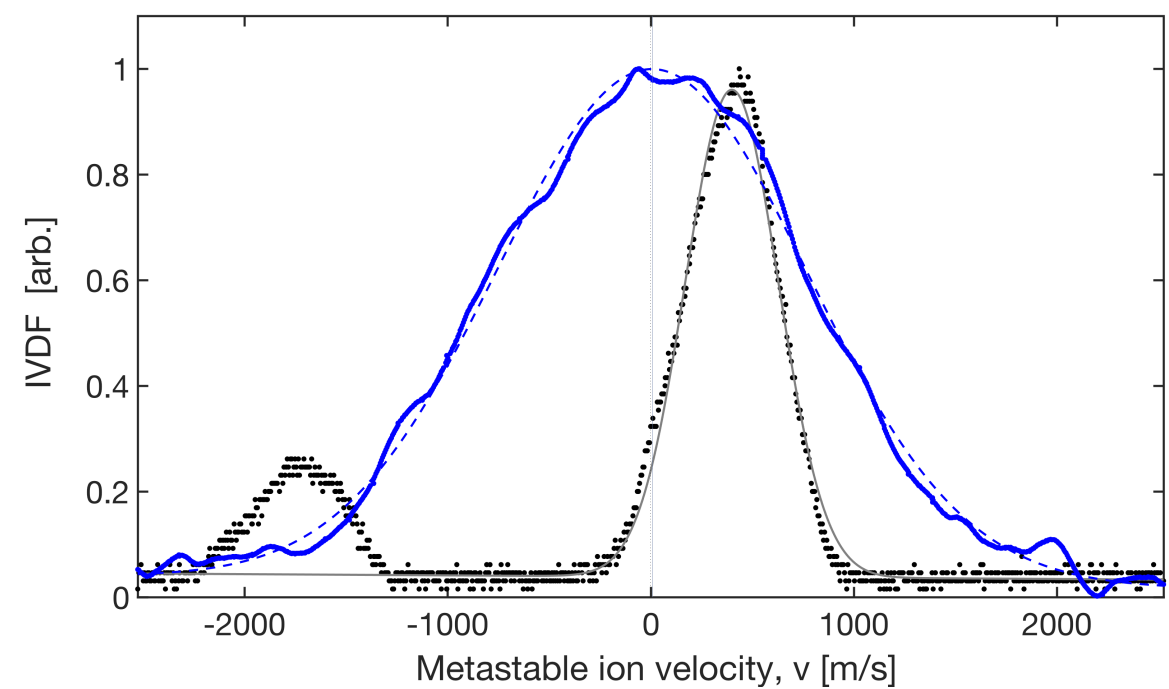

FiguRE 3.17: Sample IVDF recorded with confocal LIF in HELIX: $p=3.5$ mTorr, $P_{\mathrm{RF}}=650 \mathrm{~W}, B=600 \mathrm{G}, n \approx 5 \times 10^{18} \mathrm{~m}^{-3}, T_{e} \approx 3 \mathrm{eV}$ [146]. From this IVDF, $u_{i}=9 \mathrm{~m} / \mathrm{s}, T_{i}=0.24 \mathrm{eV}$. The argon metastable IVDF data depicted by blue dots with the best fit Maxwellian distribution according to Eq. (3.44) shown as a blue dashed line. The iodine gas reference distribution is depicted in black. Reprinted from Derek S. Thompson, Miguel F. Henriquez, Earl E. Scime, and Timothy N. Good, Review of Scientific Instruments 88, 103506 (2017), with the permission of AIP Publishing.

\section{Zeeman splitting and broadening effects}

In the presence of an external field, Zeeman splitting occurs in which the degeneracy of each argon ion (Ar-II) energy level separates into several energy levels around the unperturbed level. One group of these levels for which the magnetic orbital quantum number $m$ does not change during the transition $(\Delta m=0)$ is referred to as the $\pi$ transitions. Transitions for which $\Delta m= \pm 1$ are referred to as $\sigma_{ \pm}$transitions. In LIF, these separated levels are convolved with the wider Doppler broadening of the line and other broadening effects and create the appearance of multiple peaks in the IVDF. If the field is insufficient to make the transition groups distinct, the temperature is easily overestimated from the artificially broad IVDF.

When LIF is conducted with the laser passing across the magnetic field (i.e., for $x$ and $y$ in boundary coordinates), the $\sigma_{ \pm}$components are polarized perpendicular to the field ( $\sigma$ is shorthand for senkrecht, German for perpendicular). The laser passes through a field-aligned linear polarizer, and the $\pi$-polarized beam is not 
resonant with the $\sigma$-polarized transitions, eliminating the fluorescence from the sixteen $\sigma(\Delta m= \pm 1)$ components of the Zeeman-split spectrum.

When the laser is injected along the magnetic field, the $\Delta m=0$ transition is not observed, and the $\sigma_{ \pm}$transitions appear circularly polarized with opposite rotation [147, pp. 243-248]. Since linearly polarized light contains both right- and left-hand polarization components, unpolarized or linearly polarized light generates a bimodal fluorescence spectrum from the emission of the two $\sigma$ groups. Each peak is shifted $\Delta \nu= \pm 1.4 \mathrm{GHz} / \mathrm{kG}[143]$. A $\lambda / 4$-plate placed after the linear polarizer, oriented $45^{\circ}$ to the polarization axis of the linear polarizer, causes the linearly polarized laser to become circularly polarized. This circular polarization is resonant with only one of the $\sigma_{ \pm}$groups, selecting one of the peaks and eliminating the fluorescence from the other. The mean speed of the IVDF is at the shift-corrected location at the center of the bi-modal distribution.

Several broadening mechanisms are present in each IVDF. In addition to splitting the spectral lines, the Zeeman effect creates spectral broadening. For Ar-II in the plasma conditions of these measurements, this broadening is estimated to be $\lambda_{Z \pi} \approx 1.6 \times 10^{-4} \mathrm{~nm},<4 \%$ of the $\Delta \lambda_{D_{1 / 2}} \approx 4.4 \times 10^{-3} \mathrm{~nm}$ Doppler-broadening and is ignored in the analysis. Stark broadening $\left(O\left(10^{-6} \mathrm{~nm}\right)\right)$, line-width $\left(O\left(10^{-5}\right.\right.$ $\mathrm{nm}))$, power broadening $\left(<O\left(10^{-4} \mathrm{~nm}\right)\right)$ and pressure broadening $\left(O\left(10^{-6} \mathrm{~nm}\right)\right)$ are negligible for the Ar-II ions in these plasma conditions as well, contributing $\ll 1 \%$ to the broadening of the spectrum [144, 145]. Therefore, only Doppler broadening and Zeeman effect shifts are incorporated into analysis of the LIF data.

One type of artificial broadening derives from instrumentation instead of from atomic physics. The lock-in amplifier that the LIF signal passes through applies a time averaging window to the signal. The VDF depends on the wavelength which is swept over time, so an averaging window in time effectively applies a moving average to the VDF in velocity, which for our sweep proceeds from negative to positive velocities. The averaging window behaves as a low-pass filter. If the time constant of the filter - i.e., of the averaging window — is small compared to the 


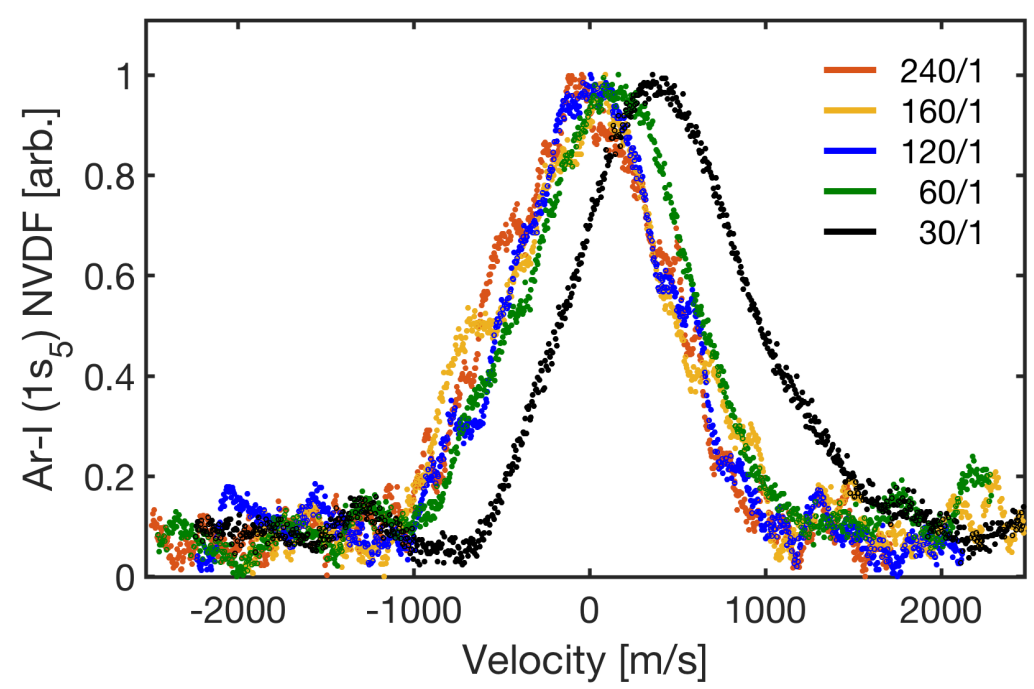

FiguRE 3.18: Example overintegration of VDFs.

scan time, the recorded VDF accurately reflects the VDF at the lock-in input, before processing. If the window is large compared to the scan time, the VDF is recorded in such a way that each point contains a significant contribution from the portions earlier in time, or equivalently lower in frequency or velocity. A VDF recorded in this way is shifted toward positive velocities. Fig. 3.18 illustrates this effect by comparing a series of NVDFs measured at the same location, laser power, and plasma conditions, but with different ratios of scan time to averaging window width, in seconds. For time constants of one second, scan times 120 seconds and longer produce an accurate, unshifted distribution. Shorter scan times introduce a noticeable artificial shift toward positive velocities, up to several hundred $\mathrm{m} / \mathrm{s}$. When the time constant is increased for noisy signals, the ratio between scan time and time constant is kept constant for consistent flow measurements. Long sweeps to mitigate overintegration easily enter the domain of diminishing returns, opposing the researcher's effort to make each measurement in the shortest time possible without loss of accuracy. A method of selecting the scan time to time constant ratio is demonstrated in Fig. 3.19. For a fixed time constant, the scan time is increased until the mean flow speed levels off at some minimum value. The scan time is selected that is shortest while not exceeding one standard deviation from the measurement of the minimum speed. For the data in Figs. 3.18 and 3.19, recorded with a one second time constant, a scan time of 120 seconds is the shortest sweep that produces a speed within a conservative $25 \mathrm{~m} / \mathrm{s}$ of the mean velocity at 


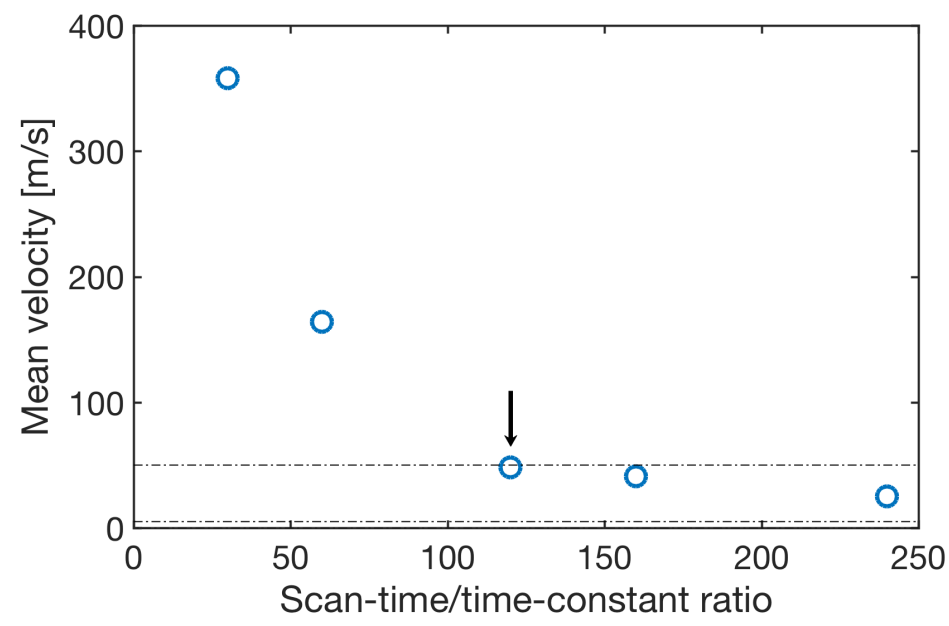

FiguRE 3.19: Overintegration trend in mean velocity for scan time selection. The arrow marks $120 \mathrm{~s}$, the selected scan time used to measure subsequent VDFs.

240 seconds.

\subsubsection{Optical arrangement}

A schematic of the optical arrangement for LIF in these experiments is shown in Fig. 3.20. Both argon metastable neutral (Ar-I) and metastable ion (Ar-II) resonances are accessed by a Sirah Matisse DR (dye ring) laser. The spectroscopic details of these resonances are described in Secs. 3.3.6 and 3.3.7. The Matisse laser provides $30 \mathrm{GHz}$ mode-hop-free sweep range and $P_{\text {las }}^{\mathrm{Ar}-\mathrm{I}} \lesssim 400 \mathrm{~mW}(\mathrm{CW})$ laser power at $706.9167 \mathrm{~nm}$ from Pyridine 1 (LDS 698) dye in a 9:1 ethylene glycol (EG): ethylene glycol monophenyl ether (EPH) solution used to fluoresce the Ar-I metastable resonance at vacuum wavelength $\lambda_{\mathrm{Ar}-\mathrm{I}}=706.913 \mathrm{~nm}$. For the Ar-II resonance at vacuum wavelength $\lambda_{\text {Ar-II }}=611.6616 \mathrm{~nm}, P_{\text {las }}^{\text {Ar-II }} \lesssim 1400 \mathrm{~mW}(\mathrm{CW})$ from Rhodamine 590 dye in ethylene glycol.

After exiting the laser cavity, $\approx 10 \%$ of the beam is separated by a $90: 10$ optical blank. $90 \%$ of the $10 \%$ separated beam passes through a stable, stationary and room temperature iodine cell. Resonance transitions in the iodine near the argon resonance act as a spectroscopic reference for the laser wavelength and corroborate the Bristol 621 wavemeter, into which the remaining $10 \%$ of the separated beam 


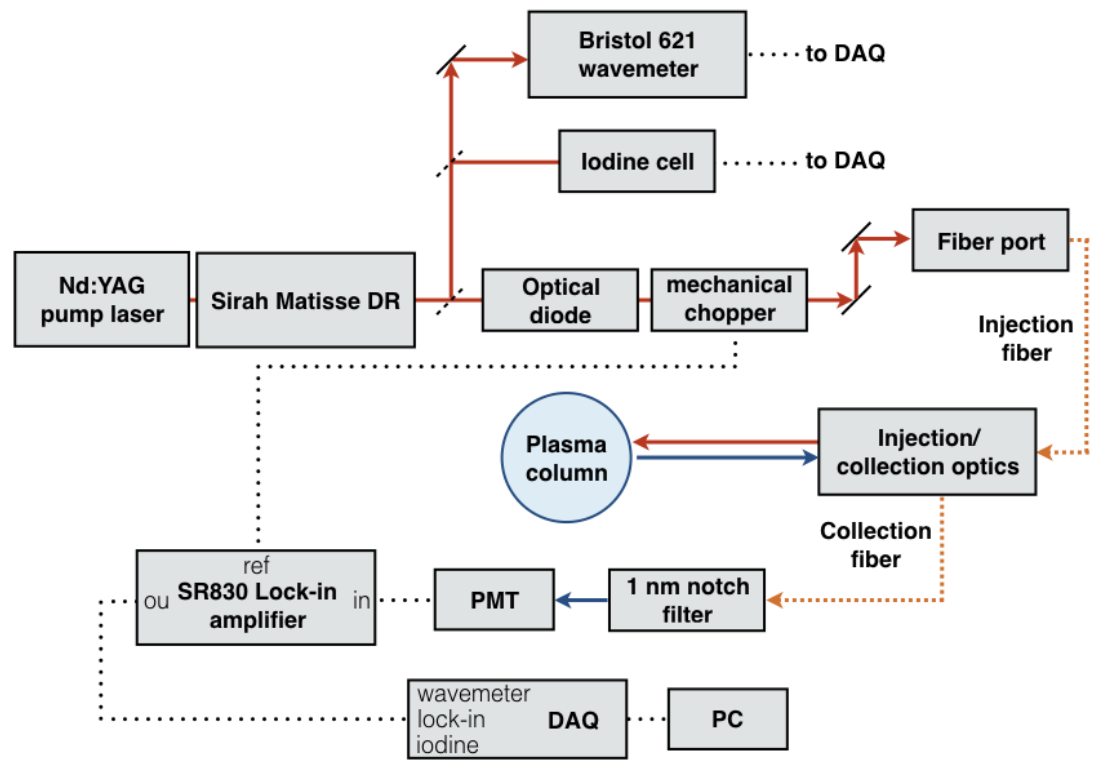

FiguRE 3.20: Optical arrangement for laser-induced fluorescence employing the Sirah Matisse dye ring laser [146]. Reprinted from Derek S. Thompson, Miguel F. Henriquez, Earl E. Scime, and Timothy N. Good, Review of Scientific Instruments 88, 103506 (2017), with the permission of AIP Publishing.

passes. The wavemeter records the wavelength and fractional power of the branch beam during the wavelength sweep, with $\Delta \lambda_{\text {las }}= \pm 0.0001 \mathrm{~nm}$ and $\Delta P_{\text {las }}^{\text {w.m. }}=$ $\pm 15 \%$.

The main beam passes through an Optics For Research IO-5-633-PBS optical diode, which prevents potentially damaging reflections from returning to the laser cavity. This diode has a specified transmittance of $73-75 \%$, however experimentally it is nearly $80 \%$. A mechanical chopper after the diode alternatively blocks and passes the beam at a frequency of $2.5-5 \mathrm{kHz}$, after which the beam is directed by turning mirrors into a fiber port. The fiber port focuses the injection beam onto the acceptance orifice of a $20 \mathrm{~m}$ long optical fiber, through which the laser is transported from the laser room to the HELIX chamber.

Two kinds of optical fiber are used. For perpendicular injection, the optical distance from the exit orifice of the fiber to the sample region is small, and a relatively large diameter collimator suffices and allows for rapid coupling between the fiber and the laser. For this application, multimode (MM) fibers are used with SMA connectors and 3-axis fiber ports. At HELIX, the fiber couples to a parabolic 
reflective collimator (ThorLabs RC04SMA-P01), which collimates the laser into a $4 \mathrm{~mm}$ diameter $\left(1 / e^{2}\right.$ distance) beam. For axial injection from the rear of HELIX, the beam travels approximately two meters before reaching the sample region and requires tighter collimation. For this application, single mode (SM) fibers are used with ThorLabs PAF-X-15-B fiber ports, which possess 5 degrees of freedom, tighter tolerances, and the greater precision of FC-type connectors. SM fibers have 5-10 $\mathrm{mm}$ cores, making them better point-source approximations than MM cores for the collimators at the expense of greater coupling difficulty and lower transmitted power. MM fibers in good condition are typically capable of $80 \%$ transmission, whereas SM fibers achieve 20-25\%. Axial injection uses a $2 \mathrm{~mm}$ diameter $\left(1 / e^{2}\right.$ distance) reflective collimator (ThorLabs RC02FC-P01) with full angle beam divergence $0.02^{\circ}$, ensuring excellent collimation over long distances. While the total power is $\approx 4 \mathrm{x}$ lower for SM fibers, the narrower beam results in comparable power density in the sample region.

Zeeman splitting occurs in both perpendicular and parallel orientations due to the magnetic fields in HELIX, as described in detail in Sec. 3.3.2. The polarization of the injected beam is altered to isolate specific components of the spectra and avoid spurious broadening. For perpendicular injection, the laser passes from the collimator through a wire-mesh gridded linear polarizer (ThorLabs WP25M-VIS). The linear polarizer transmits $80 \%$ of the laser power with a reported 800:1 extinction ratio, ensuring excellent spectral selection [148]. In the axial injection optics, a quarter wave plate (ThorLabs WPQ10ME-670; $T \approx 98 \%$ [149]) is placed after the linear polarizer, and the pair transmits slightly less than $80 \%$ of the incident beam power. (Ideally, the specified wavelength for the quarter-wave plate should match the wavelength of the laser. $\lambda=670 \mathrm{~nm}$ is the closest quarter-wave plate currently available in our lab.)

With the exception of confocal LIF (see below), the collection optics consist of a pair of simple plano-convex lenses which focus the collected fluorescence emission onto the orifice of a MM fiber, through which the fluorescence is transmitted to a photomultiplier tube (PMT). A $1 \mathrm{~nm}$ full-width at half-maximum (FWHM) notch filter isolates the fluorescence from other photon noise. (For the 461.086 
$\mathrm{nm}$ Ar-II fluorescence, maximum transmission for this filter is $\approx 72 \%$.) The PMT converts incident light to an electrical signal. A Stanford Research Systems SR830 lock-in amplifier isolates the LIF signal from electron-impact excitation and other uncorrelated noise via correlation analysis, amplifying signal at the frequency of the mechanical chopper and strongly attenuating other components. The remaining signal is recorded by a computer and is synchronized with the wavelength and power recorded by the wavemeter.

\subsubsection{Conventional LIF}

\section{$3 \mathrm{D}$ stage $-\mathrm{X}$ and $\mathrm{Y}$}

A coordinate system for the plasma boundary is introduced in Chapter 2. In this system, the measurement with LIF of $f\left(\mathbf{x}, v_{x}\right)$ and $f\left(\mathbf{x}, v_{y}\right)$ is achieved through an optomechanical arrangement with three orthogonal degrees of freedom aligned to the coordinate system of the boundary. This arrangement is referred to as the 3D stage. A rendering of the 3D stage is shown in Fig. 3.21, and its placement at the 6" cross port on HELIX is depicted in Fig. 3.22. Linear scales placed along the horizontal arm and along the vertical and axial travel of the 3D stage provide the position of the laser and collection focus once the stage is initially aligned.

A schematic of the optical arrangement of the 3D stage is shown in Fig. 3.23. The horizontal and vertical optics are attached to the translation mechanisms by dovetail mounts. Each mount holds a cage arrangement containing a complete set of optical components. The two mounts are exchanged to transition from horizontal injection (vertical collection) to vertical injection (horizontal collection) with minimal alignment and without removing the translation mechanism from the chamber. 


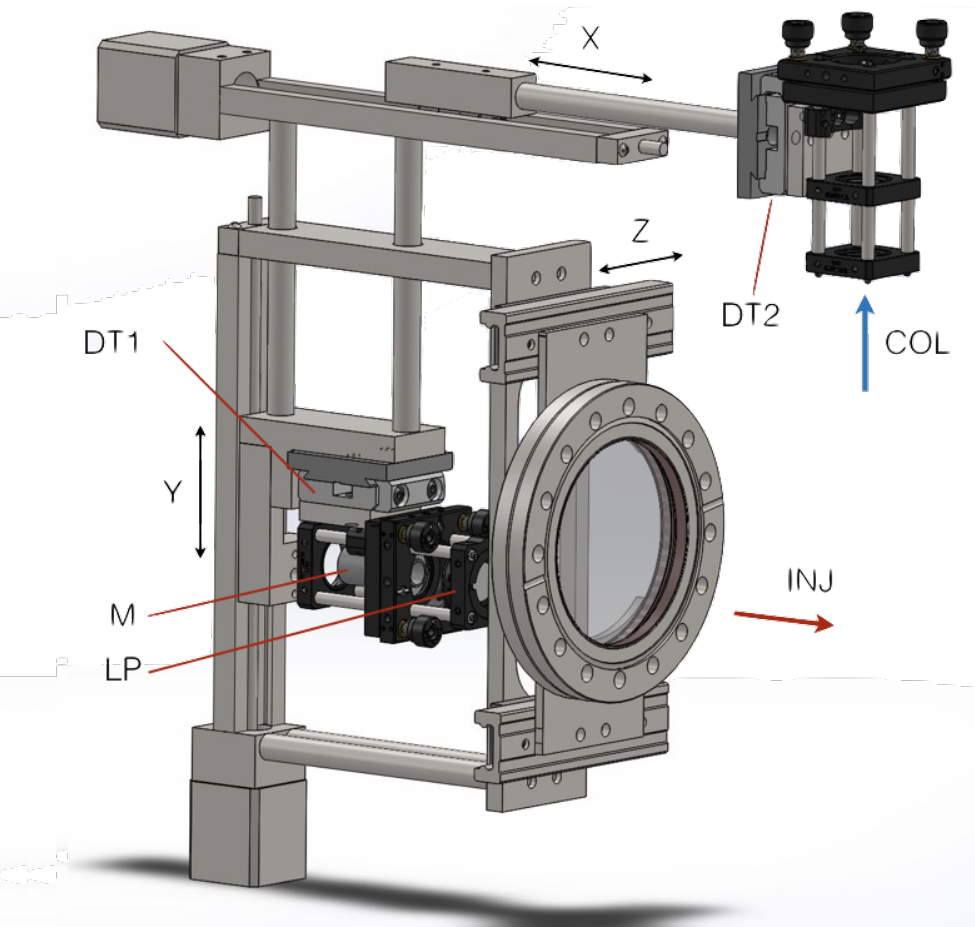

FiguRE 3.21: Rendering of the LIF 3D stage and optics, assembled for horizontal injection along $x$. The large circular flange is the side vacuum window of the HELIX chamber cross port. Translations along $x, y$ and $z$ are marked. DT1 - dovetail with injection optics; DT2 - dovetail with collection optics; M - collimating mirror (4 mm diameter beam); LP - linear polarizer; INJ - injection direction; COL - collection direction. The collection optics focus within the injection beam.

\section{Axial injection stage $-\mathrm{Z}$}

The $f\left(\mathbf{x}, v_{z}\right)$ component of the flow field near the boundary is measured using an optomechanical arrangement at the rear of the HELIX vacuum vessel. A rendering of this mechanism is shown in Fig. 3.24. The axial injection mechanism is shown in profile at the left of Fig. 3.22, with the collection optics located on the upper arm of the 3D stage, above the $6 "$ cross port as shown. The collection optics during axial injection are the same as the arrangement described in Fig. 3.23. While the XY injection and collection optics are coupled and move together, the axial injection stage and the collection optics are separate. The two stages are carefully aligned to each other whenever measuring $f\left(\mathbf{x}, v_{z}\right)$ at a new position. The position of the axial injection stage is determined using scales placed above and along the travel of the stage. The optics are mounted on a manual micrometer stage, providing redundant verification of the optical position. 


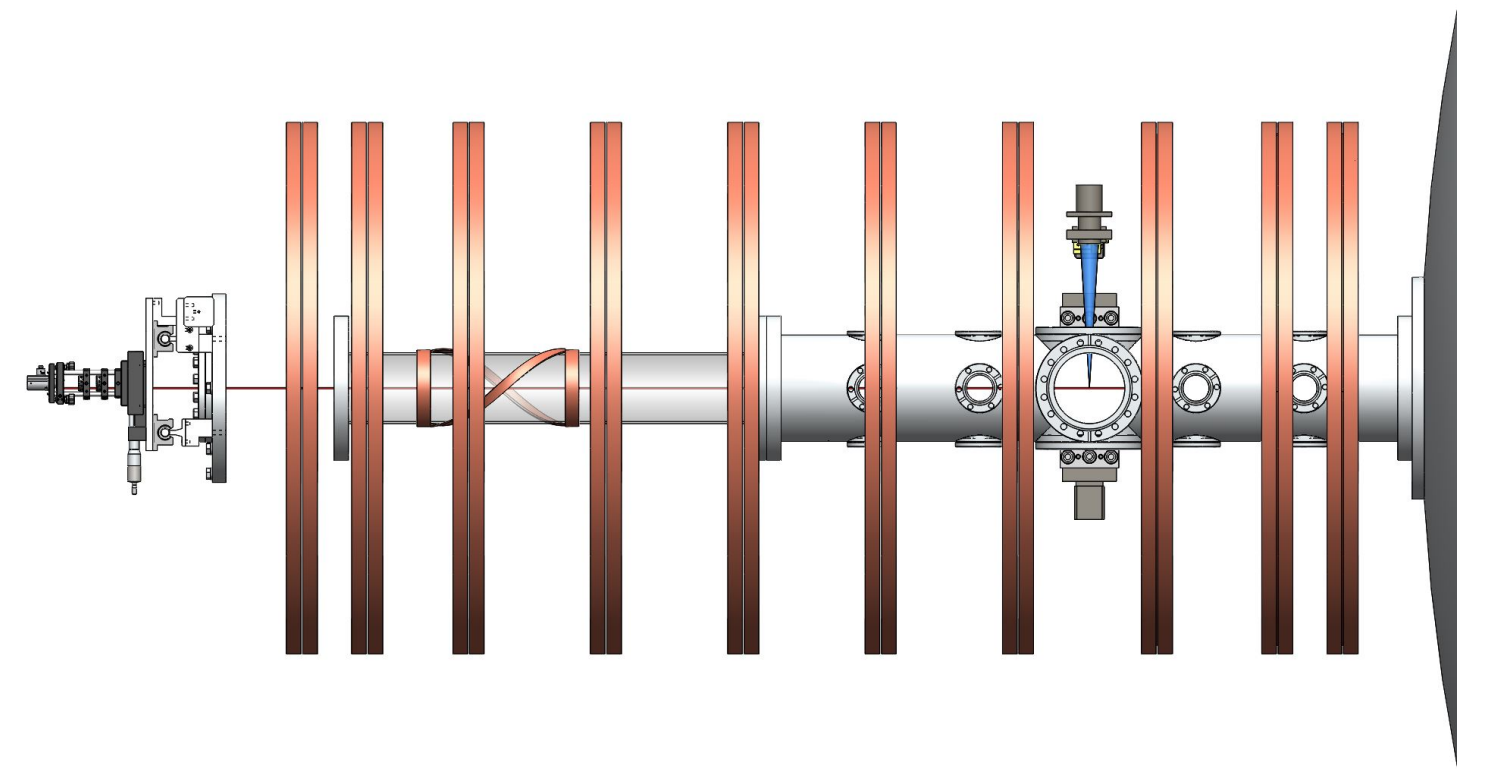

FiguRE 3.22: Rendering of the LIF axial injection optics on HELIX. The collection optics for axial injection are located at the $6 "$ cross port.

Initial alignment of the optics to the boundary is achieved in this way:

1. The laser passes through the axial stage onto a target tightly fitted to the rear window of the HELIX chamber.

2. The target is removed, and the laser is adjusted until it passes through a small window at the far end of the LEIA chamber.

3. Steps 1 and 2 are repeated until the laser is both centered on the target and passes through the LEIA window. The axial laser now defines the chamber axis. The scale positions are zeroed, and the micrometer position is recorded.

4. The boundary is inserted until it appears at the center of the upper window of the $6 "$ cross port.

5. The collection fiber is backlit using a pen laser and centered onto a target on the upper window of the cross port.

6. The target is removed and replaced until the axial laser and collection spot overlap at the center of the boundary at the same time that the collection spot is centered on the window target. 


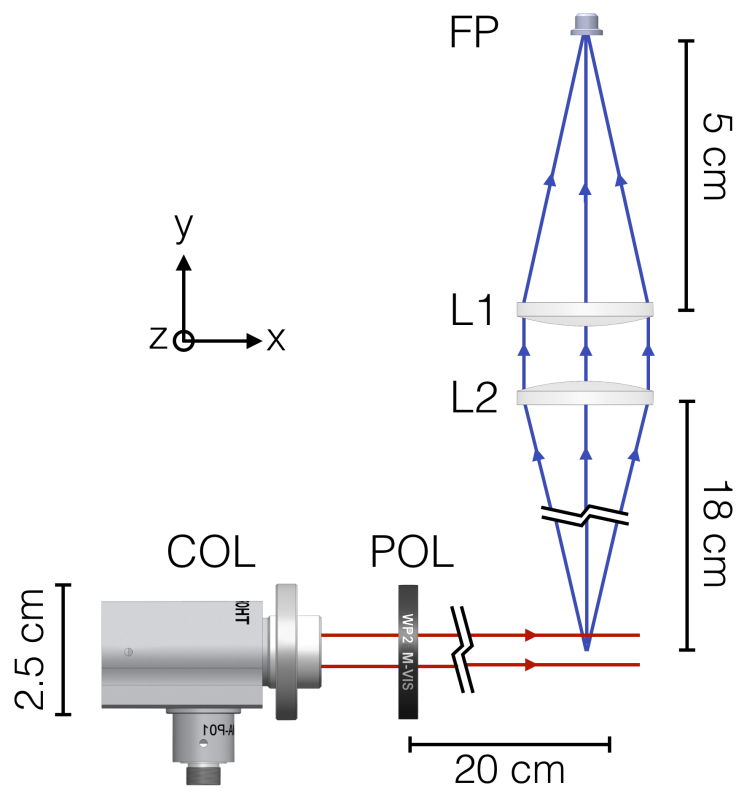

Figure 3.23: Schematic of the 3D stage optics indicating the optical paths and coordinate system during horizontal injection along $x$ [146]. Red lines show the path of the injection laser. Blue lines show the path of the collected fluorescence. FP - Fiber port for multimode collection fiber; L1 $-f=50 \mathrm{~mm}$ plano-convex lens; L2 $-f=200 \mathrm{~mm}$ plano-convex lens; COL - reflective collimator; POL - wire grid linear polarizer. Reprinted from Derek $S$. Thompson, Miguel F. Henriquez, Earl E. Scime, and Timothy N. Good, Review of Scientific Instruments 88, 103506 (2017), with the permission of AIP Publishing.

7. A third laser beam passes through the horizontal injection optics so that it intersects the vertical and axial beams.

8. When all three beams intersect at the origin of the boundary coordinate system, the Velmex translation stage zeros are set, and all scale measurements are verified.

Before each IVDF is recorded, the linear scale positions are recorded with the IVDF identification number electronically on the lab computer and in at least one lab notebook.

\subsubsection{Confocal LIF}

The LIF optical arrangements described in the previous two sections benefit from an experimental luxury: two available, orthogonal windows in the chamber wall 


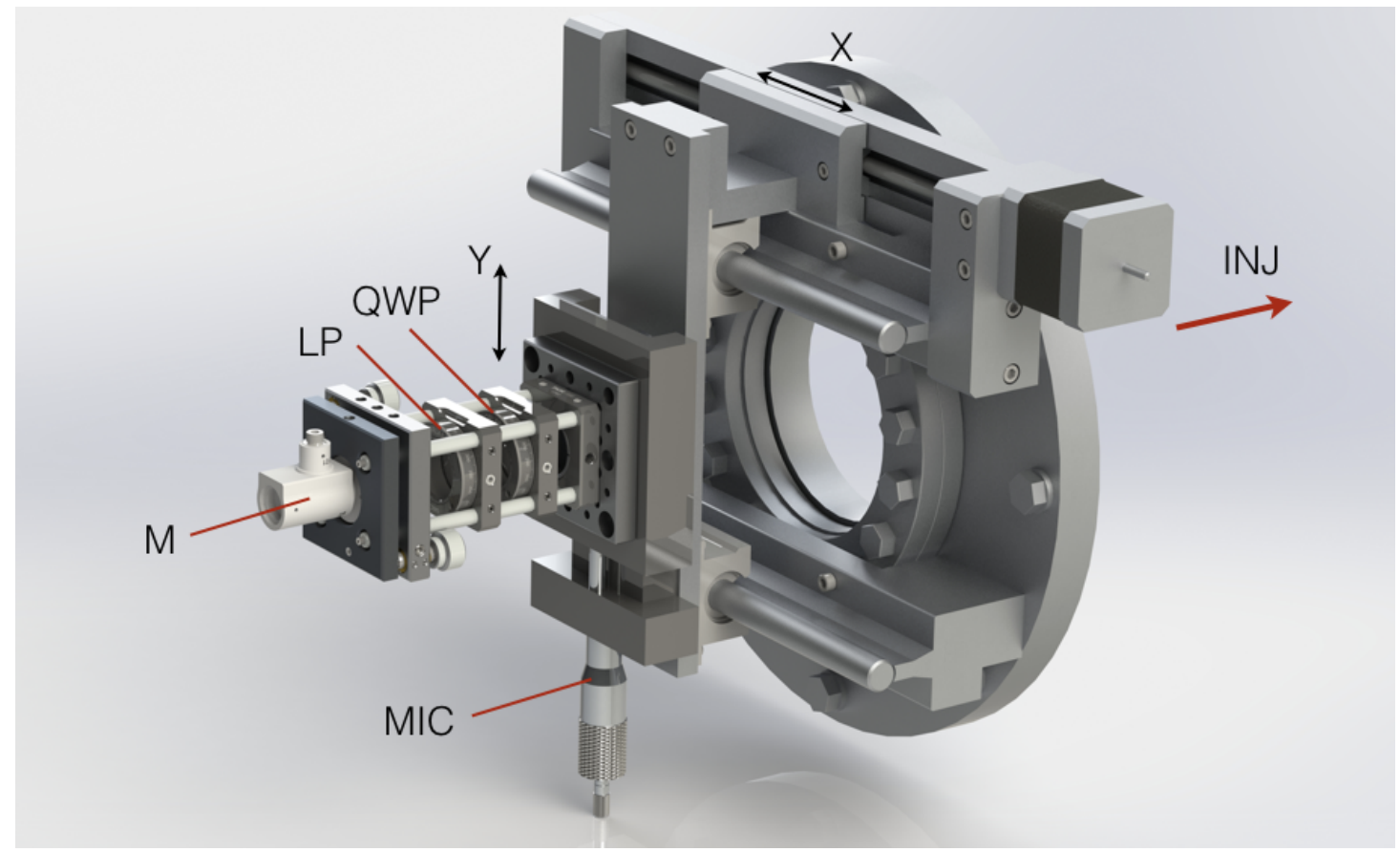

FiguRE 3.24: Rendering of the axial LIF injection optics. The large circular flange is the rear vacuum wall of the HELIX chamber. $x$ and $y$ translation directions are marked. $\mathrm{M}$ - reflective collimator (2 mm diameter beam); LP linear polarizer; QWP - $\lambda / 4$-plate; MIC - vertical micrometer stage; INJ injection direction toward HELIX.

that provide access to the sample region. Many experiments do not offer multiple windows in this configuration. A new way to conduct LIF through a single small window alleviates these diagnostic access issues.

Satisfying the single window constraint requires a new optical design in which the injection and collection paths overlap at the focus and are concentric between the optical assembly and the focal point. We refer to this design as the confocal fluorescence telescope and to this diagnostic as confocal laser-induced fluorescence [146]. The two window arrangement may be referred to as perpendicular or conventional LIF. Fig. 3.25 shows a schematic of the optical paths in confocal LIF, and Fig. 3.26 shows the confocal optics in place on HELIX. The laser and data acquisition arrangement is the same as the one described in Fig. 3.20.

Confocal LIF uses an SM fiber (5-10 $\mu \mathrm{m}$ core) connected to a reflective collimator (2 mm diameter beam) to create a very tight focus of the injection beam. The beam passes through the same wire-mesh gridded linear polarizer that is 


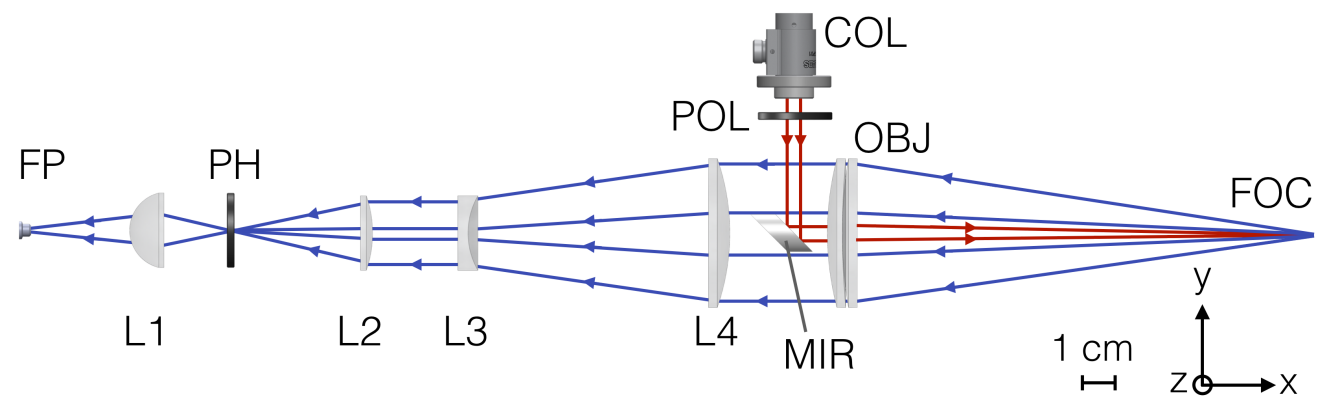

FIgURE 3.25: A schematic of the confocal optical assembly with coordinate directions marked. Red lines show the path of the injection laser. Blue lines show the path of the collection optics. FP - multimode fiber port, L1 $-f=25$ $\mathrm{mm}$ plano-convex lens, $\mathrm{PH}-$ pinhole, $\mathrm{L} 2-f=75 \mathrm{~mm}$ plano-convex lens, L3 $f=-50 \mathrm{~mm}$ plano-concave, $\mathrm{L} 4-f=300 \mathrm{~mm}$ plano-convex lens, $\mathrm{COL}-$ reflective collimator, POL - wire grid linear polarizer, MIR $-45^{\circ}$ elliptical mirror, OBJ - plano-convex/meniscus objective doublet, FOC - shared injection/collection focus [146]. Reprinted from Derek S. Thompson, Miguel F. Henriquez, Earl E. Scime, and Timothy N. Good, Review of Scientific Instruments 88, 103506 (2017), with the permission of AIP Publishing.

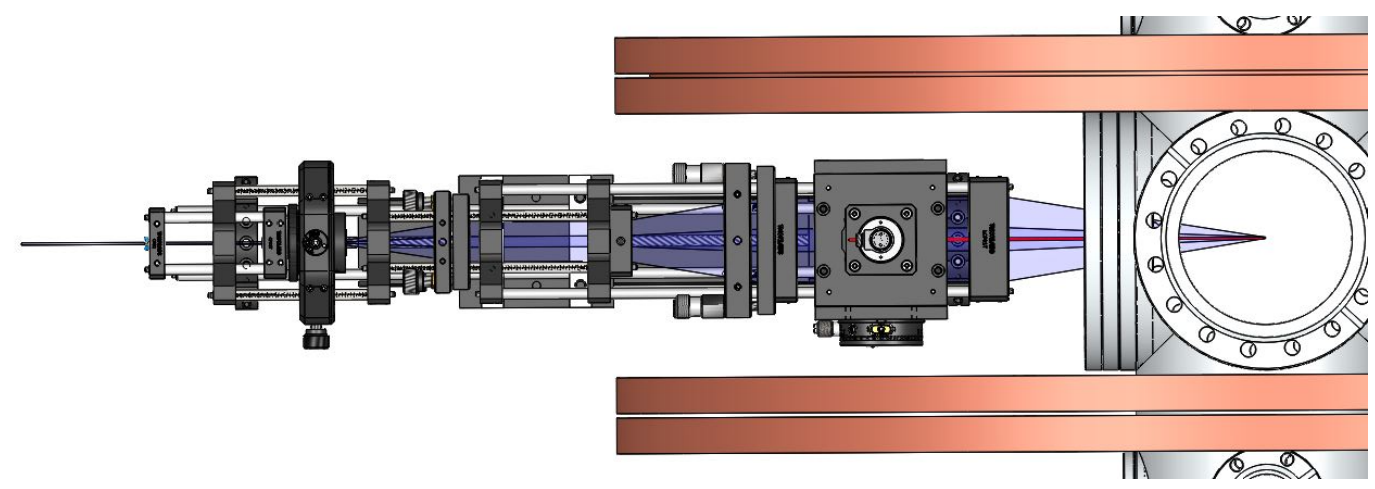

Figure 3.26: A top-down view rendering of the confocal optical assembly deployed on HELIX.

used in conventional LIF. The polarized beam reflects off a $12.7 \mathrm{~mm}$ minor diameter 45-degree elliptical turning mirror, which is oriented so that it presents a circular profile when viewed down the optical axis. The beam exits through an anti-reflection coated N-BK7 $50.8 \mathrm{~mm}$ diameter plano-convex objective lens paired with a positive meniscus lens that sets the focal length and therefore the sample volume location of the diagnostic. This doublet mitigates chromatic and spherical aberration. When used on HELIX, the two lenses have a measured combined focal length $f=149 \mathrm{~mm}$. The polarization of the injection beam is confirmed by placing a second linear polarizer in the optical path. The transmitted power when the two polarization axes are normal is observed to be $\ll 1 \%$ of the maximum when they 
are parallel, confirming that the polarization has been preserved by the elliptical mirror and objective optics.

A subsection of the solid angle of the fluorescence passes back through the optical assembly. A series of lenses reduces the diameter of the ray bundle and focuses the collected light onto a pinhole spatial filter at the back of the optical assembly. The pinhole size may be optimized for depth of field and diffraction-limited use, which suggests a smaller diameter should be used [150]. That said, a diameter of $50 \mu \mathrm{m}$ is found to provide excellent depth of field and throughput for our needs. The light exiting the pinhole is condensed by a final lens onto the orifice of an NA $=0.22,200 \mu \mathrm{m}$ diameter core multimode fiber, that transmits it to the PMT.

Absolute and relative density measurements require consistency in the diagnostic between IVDFs recorded at different positions so that only the plasma influences the result. Beside the consistently overlapping injection and collection focus, several factors contribute to variations in intensity. The PMT bias and the settings of the lock-in amplifier remain unchanged through the measurements. The wavelength dependent transmittance variation of N-BK7 and the anti-reflective coating during the laser sweep is estimated to be $\delta T<0.005 \%$ and is neglected. The laser power also varies slightly with wavelength. For single-photon LIF the integrated spectrum should increase linearly with increasing laser power, before plateauing when the absorption saturates. For the plasma conditions in which the confocal LIF data were recorded, the linear dependence is observed, and no plateau is seen even at the highest power output of the laser.

An example IVDF, measured confocally in a 3.5 mTorr, $650 \mathrm{~W}$ helicon plasma with $B=600 \mathrm{G}$, is shown in Fig. 3.17 with its reference iodine spectrum. The IVDF indicates an essentially unmoving mean flow and ion temperature $T_{i}=0.24$ $\mathrm{eV}$. The SNR of this IVDF $-\mu_{\mathrm{sig}} / \sigma_{\text {nse }}$, the ratio of the signal average to the standard deviation of the noise - is calculated to be approximately 15. The SNR for conventional LIF under the same conditions is 22, indicating that at least $70 \%$ of the conventional SNR can be obtained by the confocal technique. 

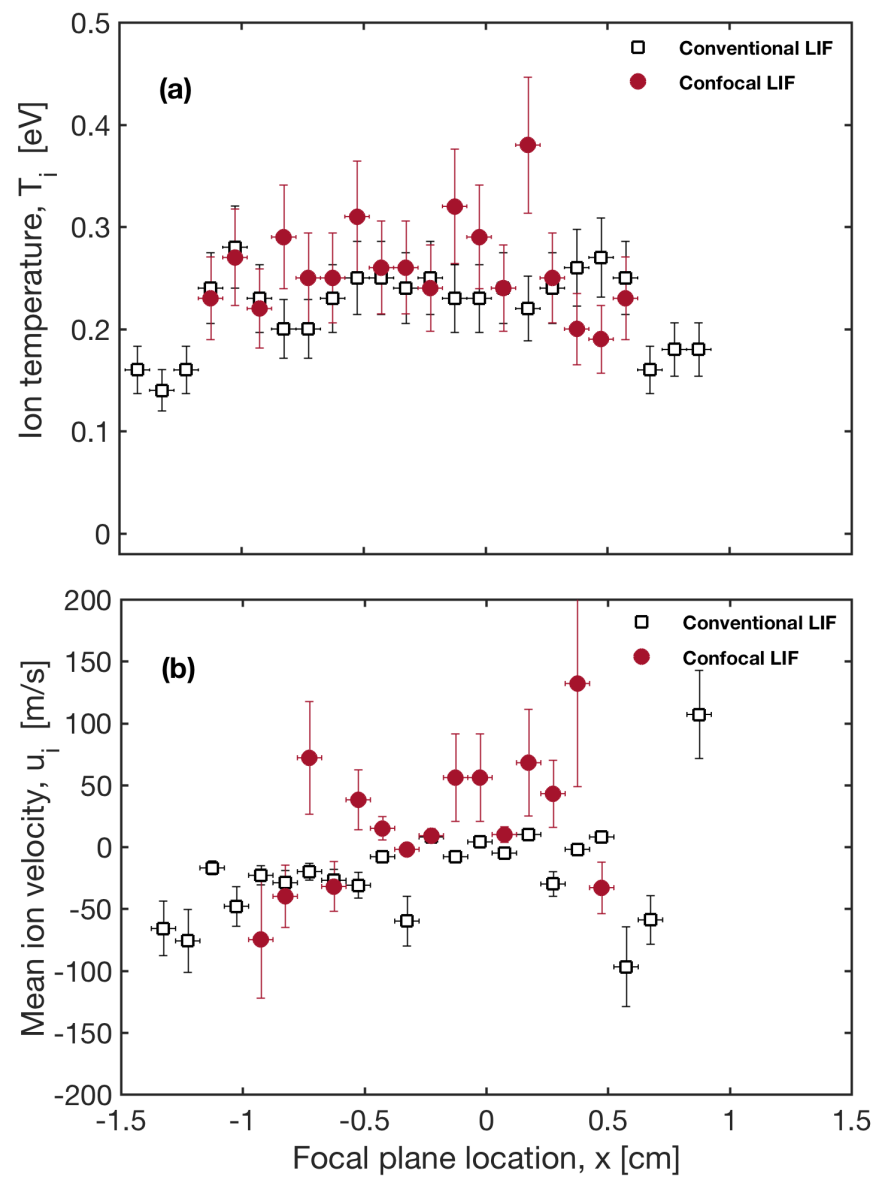

FiguRE 3.27: (a) Ion temperature and (b) mean flow speed profiles measured with conventional and confocal LIF [146]. Reprinted from Derek S. Thompson, Miguel F. Henriquez, Earl E. Scime, and Timothy N. Good, Review of Scientific Instruments 88, 103506 (2017), with the permission of AIP Publishing.

Temperature and flow profile comparisons are shown in Fig. 3.27. The horizontal error reflects the $0.5 \mathrm{~mm}$ uncertainty in the linear scale position used to identify the position of the focus. The vertical error derives from error in the Maxwellian fit to the IVDF and from shot-to-shot variation. In the central region of the plasma, the flow is consistent with zero, with $u^{\text {conf. }}=21 \pm 53 \mathrm{~m} / \mathrm{s}, u^{\text {conv. }}=-21 \pm 41 \mathrm{~m} / \mathrm{s}$, $\bar{T}_{\mathrm{i}}^{\text {conf. }}=0.26 \pm 0.05 \mathrm{eV}$, and $\bar{T}_{\mathrm{i}}^{\text {conv. }}=0.23 \pm 0.03 \mathrm{eV}$, confirming that conventional and confocal LIF produce comparable results. The error is primarily statistical.

The focal point and sample volume of the confocal optical arrangement is determined by taking profiles of the injection and backlit collection optics using a Newport LBP HR beam profiler. The beam profiler is a $7.6 \mathrm{~mm} \times 6.2 \mathrm{~mm}$ active area charge-coupled device (CCD) with $4.65 \mu \mathrm{m} \times 4.65 \mu \mathrm{m}$ pixels. Imaging the 


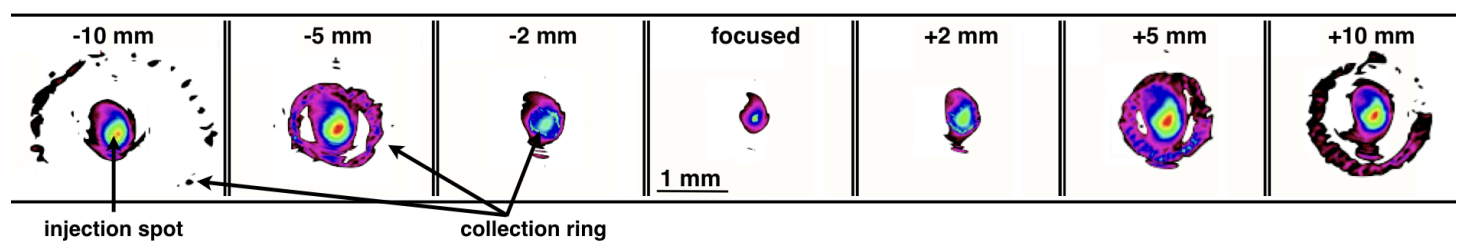

Figure 3.28: Beam profiles showing the shape, focus and overlap of the injection and collection ray bundles [146]. Reprinted from Derek S. Thompson, Miguel F. Henriquez, Earl E. Scime, and Timothy N. Good, Review of Scientific Instruments 88, 103506 (2017), with the permission of AIP Publishing.

shape of the ray bundle for each optical path provides the location and distance to the overlap region of the two bundles. Fig. 3.28 shows a series of beam profiles showing the shape, focus, and overlap of the injection and collection volumes several millimeters around the focal plane. Negative distances correspond to planes between the optics and the focal plane. Away from the focal plane, the elliptical mirror creates a central obscuration that causes the collection ray bundle profile to form an annulus whose radius decreases toward the focus. In a finite region around the focal plane, the collection and injection ray bundles overlap. It is from this region that the collected LIF signal originates. The optical response of the confocal LIF technique describes the spatial distribution of the light that contributes to the signal for a flat density. The width of this optical response provides a measure of how well the confocal optics isolate signal from the intended sample volume and exclude signal from elsewhere. At $f=149 \mathrm{~mm}$, this optical response has a FWHM of $1.4 \mathrm{~mm}$, from which $65.8 \%$ of the signal originates. This width is about $30 \%$ broader than conventional LIF, which has a FWHM of $1.1 \mathrm{~mm}$ and encompasses $97.3 \%$ of the signal. Previously published state-of-the-art confocal LIF designs exhibit collection regions several centimeters wide, leading to flattened profiles of measured plasma quantities [151]. By comparison, the excellent agreement between the profiles measured confocally and in the conventional way, even on the far side of the core at $x>0$ as shown in Fig. 3.29, demonstrates that the spatial localization of the two methods is similar. 


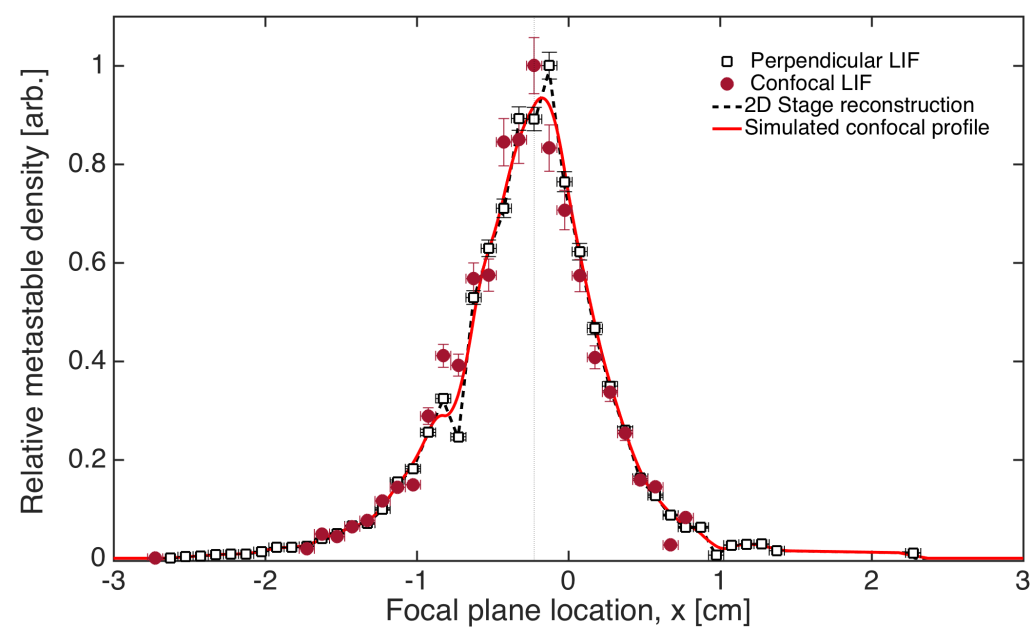

FiguRE 3.29: Relative metastable density profiles comparing the resolution of confocal and conventional LIF. $P_{\mathrm{RF}}=650 \mathrm{~W}, B=600 \mathrm{G}, n \approx 5 \times 10^{18} \mathrm{~m}^{-3}$, $T_{e} \approx 3 \mathrm{eV}$ [146]. Reprinted from Derek S. Thompson, Miguel F. Henriquez, Earl E. Scime, and Timothy N. Good, Review of Scientific Instruments 88, 103506 (2017), with the permission of AIP Publishing.

\subsubsection{Ar-I LIF}

Published LIF schemes for measuring neutral argon velocity distribution functions (NVDFs) have existed since the mid-1980s [152]. They were developed further in the subsequent decades to access multiple transitions and observe multiple directions simultaneously [153-155]. Ar-I LIF with diode lasers was demonstrated in the early 2000s [143] and was combined with a collisional-radiative model to extract absolute densities [80]. In the scheme employed in Refs. [143] and [80], the laser scans over the $667.9125 \mathrm{~nm}$ resonance to pump the $4 s\left({ }^{2} P_{3 / 2}^{0}\right)_{1}$ non-metastable state to the $4 p^{\prime}\left({ }^{2} P_{1 / 2}^{0}\right)_{0}$ state, which decays to the $4 s^{\prime}\left({ }^{2} P_{1 / 2}^{0}\right)_{1}$ state by emitting a $750.5934 \mathrm{~nm}$ photon. In Paschen notation this is the $1 s_{4} \rightarrow 2 p_{1} \rightarrow 1 s_{2}$ excitationdecay transition sequence. While this pump transition is accessible through several available dye and diode lasers, it has the disadvantage of requiring a sufficient population in the non-metastable $1 s_{4}$ state, which is primarily populated by the electron-impact excitation of atoms occupying the nearby $1 s_{5}$ and $1 s_{3}$ metastable states. Recently, a new $1 s_{2}$ Ar-I LIF scheme demonstrated improved signal-tonoise over previous approaches that pumps the $1 s_{4}$ state by taking advantage of the improved sensitivity photomultiplier detectors have to the visible range of the $706.9167 \mathrm{~nm} 2 p_{3} \rightarrow 1 s_{5}$ fluorescence [156]. In Ref. [155], the metastable $1 s_{5}$ state 


\begin{tabular}{cccc}
\hline \hline Upper state & $\lambda_{12}[\mathrm{~nm}]$ & Decay state & $\lambda_{23}[\mathrm{~nm}]$ \\
\hline $4 p^{\prime}\left[{ }^{2} P_{1 / 2}^{0}\right]_{1}\left(2 p_{2}\right)$ & 696.7352 & & \\
& & $\left.4 s{ }^{2} P_{3 / 2}^{0}\right]_{1}\left(1 s_{4}\right)$ & $\mathbf{7 2 7 . 4 9 4 0}$ \\
& & $\left.\left.4 s^{\prime}\right|^{2} P_{1 / 2}^{0}\right]_{0}\left(1 s_{3}\right)$ & 772.6333 \\
$4 p^{\prime}\left[{ }^{2} P_{3 / 2}^{0}\right]_{2}\left(2 p_{3}\right)$ & 706.9167 & & 826.6794 \\
& & $\left.\left.4 s^{\prime}\right|^{2} P_{1 / 2}^{0}\right]_{1}\left(1 s_{2}\right)$ & \\
$4 p^{\prime}\left[{ }^{2} P_{3 / 2}^{0}\right]_{1}\left(2 p_{4}\right)$ & 714.9012 & $\left.4 s^{\prime} \mid{ }^{2} P_{1 / 2}^{0}\right]_{1}\left(1 s_{2}\right)$ & $\mathbf{7 9 5 . 0 3 6 2}$ \\
& & $\left.\left.4 s\right|^{2} P_{3 / 2}^{0}\right]_{1}\left(1 s_{4}\right)$ & 852.3783 \\
& & $\left.\left.4 s^{\prime}\right|^{2} P_{1 / 2}^{0}\right]_{1}\left(1 s_{2}\right)$ & $\mathbf{8 4 1 . 0 5 2 1}$ \\
\hline \hline
\end{tabular}

TABLE 3.6: Summary of Ar-I metastable $4 s\left[{ }^{2} P_{3 / 2}^{0}\right]_{2}\left(1 s_{5}\right)$ LIF schemes with VIS/NIR transitions. Fluorescence lines observed are set in bold.

is pumped directly. However, this is accomplished by exciting to the $3 p_{2}$ state through the resonance at $395 \mathrm{~nm}$, beyond the range of the Toptica DL100 and the Sirah Matisse DR with Rhodamine and Pyridine 1 dye [155]. Relative densities of the $1 s_{5}$ state were observed to exceed the densities of other first level Ar-I excitations $\left(1 s_{4}, 1 s_{3}\right.$, and $\left.1 s_{2}\right)$ by an order of magnitude in inductive plasmas [154].

For these experiments, we explored three schemes that take advantage of the highly populated $1 s_{5}$ metastable state and the high output power of the Sirah Matisse dye ring laser. Fig. 3.30 shows a Grotrian energy level diagram of the pump and fluorescence sequences used for probing the $1 s_{4}$ and $1 s_{5}$ states. In these schemes, the metastable $4 s\left({ }^{2} P_{3 / 2}^{0}\right)_{2}\left(1 s_{5}\right)$ state is pumped to one of the states $4 p^{\prime}\left({ }^{2} P_{1 / 2}^{0}\right)_{1}$, $4 p^{\prime}\left({ }^{2} P_{3 / 2}^{0}\right)_{2}$, and $4 p^{\prime}\left({ }^{2} P_{3 / 2}^{0}\right)_{1}\left(2 p_{2}, 2 p_{3}\right.$, and $2 p_{4}$, respectively). These transitions occur at $696.7352 \mathrm{~nm}, 706.9167 \mathrm{~nm}$, and $714.9012 \mathrm{~nm}$. The upper states have several decay paths, all of which occur in the red to near infrared wavelength range. The details of these transitions are shown in Tab. 3.6.

Where the Ar-II and Ar-I $\left(1 s_{4}\right)$ fluorescence is filtered by passing through a thin film bandpass filter, the $1 s_{5}$ fluorescence is passed through a McPherson Model 209 1.33 m Czerny-Turner high resolution monochrometer. The monochrometer acts as a filter with a $\pm 5 \mathrm{~nm}$ pass band [157]. During initial setup, light from a NIST standard lamp is transmitted through an optical fiber and coupled to the 


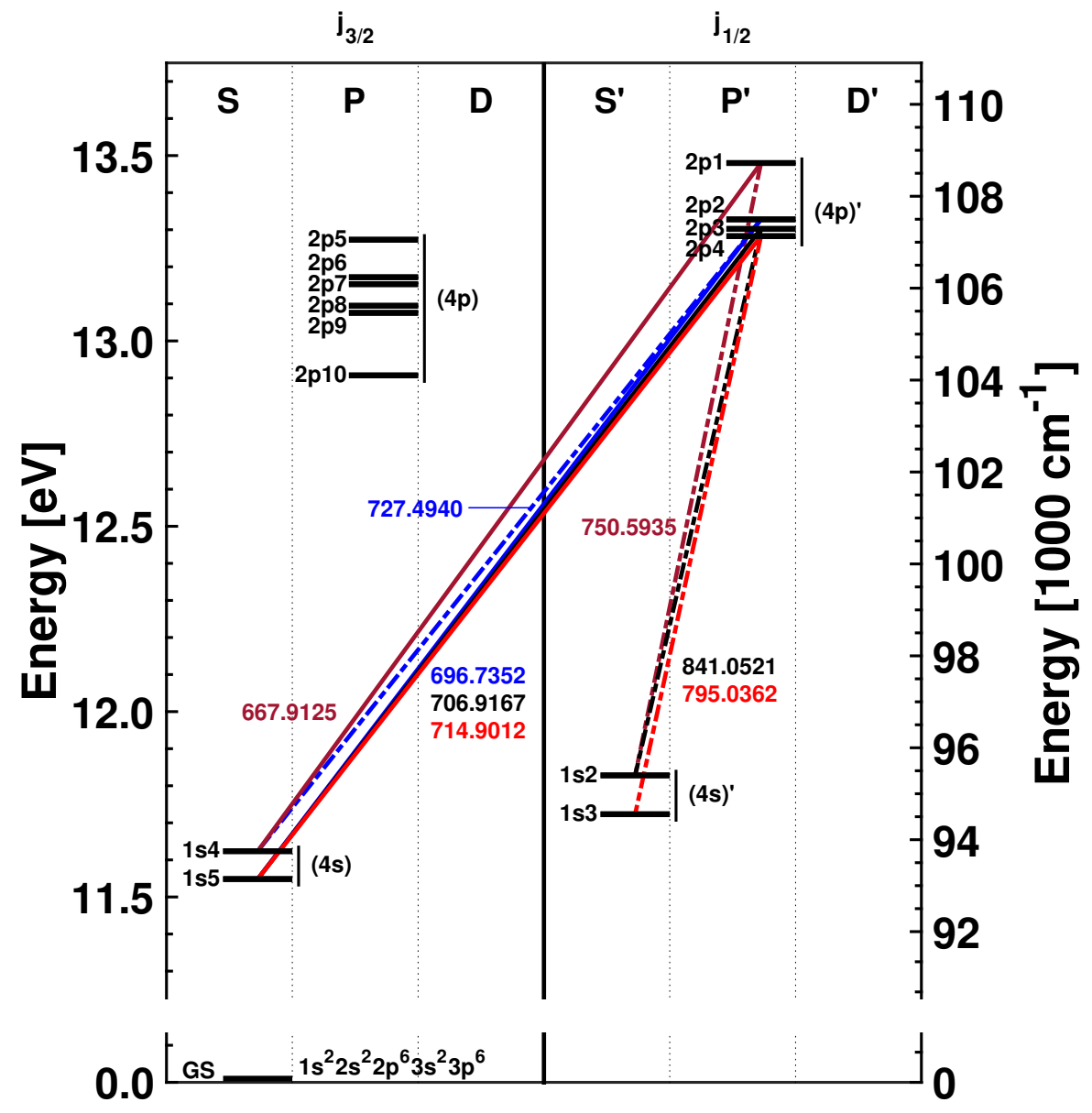

FiguRE 3.30: Grotrian diagram for metastable Ar-I LIF showing $1 s_{5}$ and $1 s_{4}$ transitions [25]. (C) 2018 IOP Publishing. Reproduced with permission. All rights reserved.

entrance slit of the spectrometer. A CCD records the dispersed spectrum around the fluorescence wavelength. Once the target wavelength is centered on the camera, the standard lamp is exchanged for the fluorescence from the plasma, and a mirror in the spectrometer cavity is turned so that the light reflects instead into an IR sensitive PMT. The PMT signal passes to the lock-in amplifier and is recorded to the lab computer.

Typical Ar-I spectra recorded in an inductive plasma $-B=450 \mathrm{G}, P_{\mathrm{rf}}=400$ $\mathrm{W}, p=6$ mTorr - with injection perpendicular to the magnetic field are shown in Fig. 3.31. 

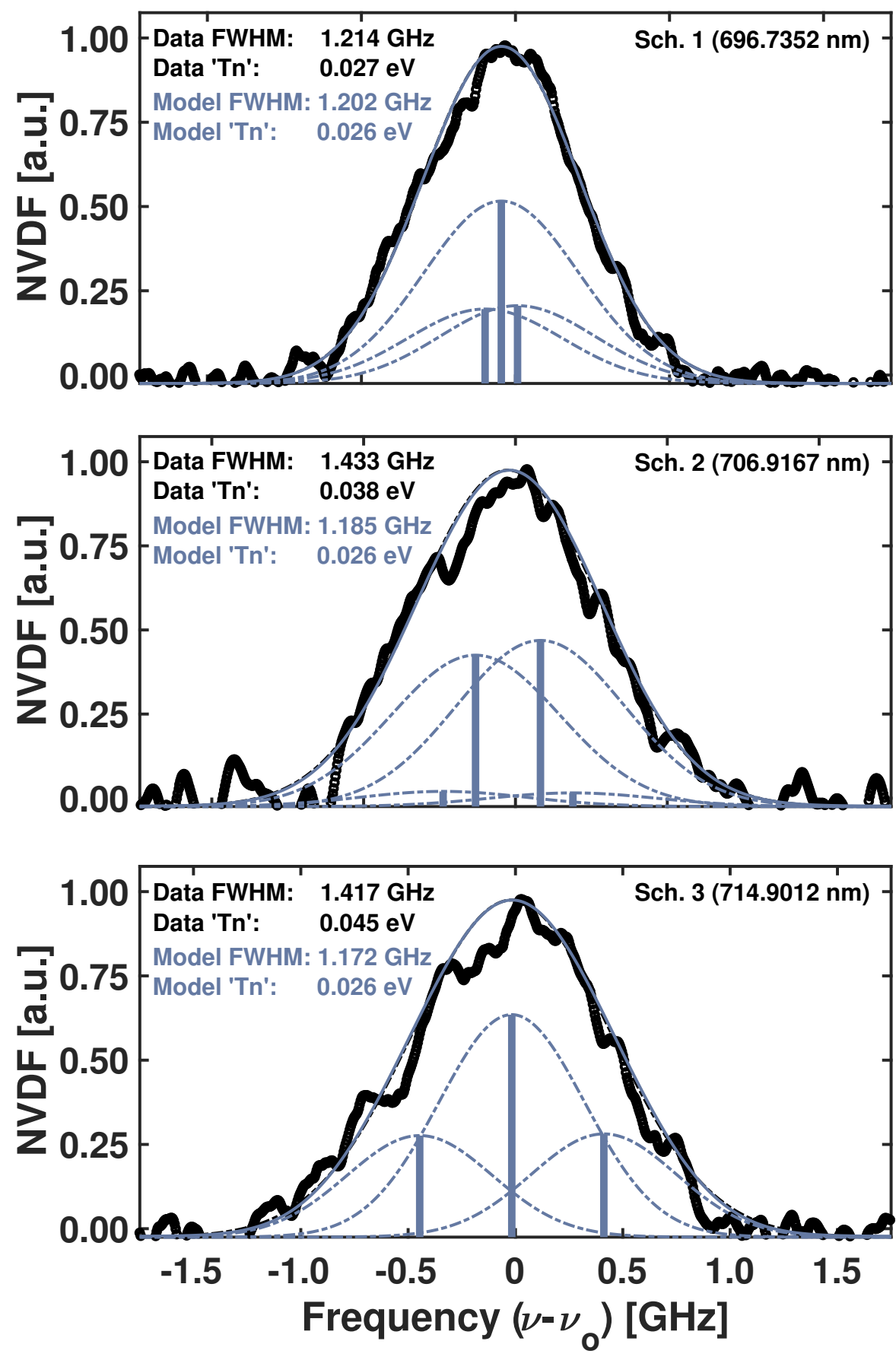

FiguRE 3.31: Example Ar-I $1 s_{5}$ spectra recorded on-axis in an RF plasma: $B$ $=450 \mathrm{G}, P_{\mathrm{rf}}=400 \mathrm{~W}, p=6 \mathrm{mTorr}, P_{\text {las }}=3 \mathrm{~mW}$. Modeling of the spectra using theoretical Zeeman splitting provides an estimate of the neutral temperature once Zeeman effects are removed [25]. (C) 2018 IOP Publishing. Reproduced with permission. All rights reserved.

Assuming a single neutral temperature and fitting these data to Eq. (3.43), the three schemes produce significantly different temperatures: $T_{n}=0.027 \mathrm{eV}$ for the $2 p_{2}$ scheme at $696.7352 \mathrm{~nm}, T_{n}=0.038 \mathrm{eV}$ for the $2 p_{3}$ scheme at $706.9167 \mathrm{~nm}$, and $T_{n}=0.045 \mathrm{eV}$ for the $2 p_{4}$ scheme at $714.9012 \mathrm{~nm}$. In fact, it is inappropriate 


\begin{tabular}{cccc}
\hline \hline Upper state & $\begin{array}{c}\delta \nu_{i}(\pi) \\
{[\mathrm{GHz}]}\end{array}$ & $\begin{array}{c}\text { FWHM, } \Delta \nu_{\mathrm{Z} \pi} \\
{[\mathrm{MHz} / \mathrm{mT}]}\end{array}$ & $\begin{array}{c}\text { FWHM, } \Delta \nu_{\mathrm{Z} \sigma} \\
{[\mathrm{MHz} / \mathrm{mT}]}\end{array}$ \\
\hline $2 p_{2}(696.7352 \mathrm{~nm})$ & $0, \pm 0.168$ & 4 & 5 \\
$2 p_{3}(706.9167 \mathrm{~nm})$ & $\pm 0.1512, \pm 0.3023$ & 21 & 12 \\
$2 p_{4}(714.9012 \mathrm{~nm})$ & $0, \pm 0.4289$ & 25 & 30 \\
\hline \hline
\end{tabular}

TABle 3.7: Summary of Ar-I metastable $4 s\left[{ }^{2} P_{3 / 2}^{0}\right]_{2}\left(1 s_{5}\right)$ Zeeman effect details. Courtesy of Amy M. Keesee, personal communications.

to refer to these values as the temperature. Instead, they are simply the spectral full width half maximum (FWHM). This disparity is due to the different internal Zeeman splitting of each transition. To model these transitions, the data are fit by Eq. (3.45):

$$
I(\nu)=\alpha_{\mathrm{DC}}+\sum_{i=1}^{n} I_{i} \exp \left[-\gamma\left(\nu-\nu_{o}-\left(u_{\mathrm{n}} / c\right) \nu_{o}-\delta \nu_{i}\right)^{2}\right]
$$

In Eq. (3.45), $T_{\mathrm{n}}$ is assumed to be the same for all sub-distributions. The spectral shifts $\delta \nu_{i}$ from the Zeeman effect are calculated from atomic theory [158] and provided in Tab. 3.7 .

The spectral widths of the sub-distributions now correspond to "temperatures" that are similar: $T_{n}=0.026 \mathrm{eV}$ for the $2 p_{2}$ scheme at $696.7352 \mathrm{~nm}, T_{n}=0.026$ $\mathrm{eV}$ for the $2 p_{3}$ scheme at $706.9167 \mathrm{~nm}$, and $T_{n}=0.026 \mathrm{eV}$ for the $2 p_{4}$ scheme at $714.9012 \mathrm{~nm}$. These numbers are within error for the measurement, which is $\approx 0.002 \mathrm{eV}$. The disparity in spectral width is predicted at least qualitatively by the FWHM predicted for the $\pi$ transitions, $\Delta \nu_{\mathrm{Z} \pi}$, shown in Tab. 3.7, for which $\Delta \nu_{\mathrm{Z} \pi}\left(2 p_{4}\right) \gtrsim \Delta \nu_{\mathrm{Z} \pi}\left(2 p_{3}\right)>6 \Delta \nu_{\mathrm{Z} \pi}\left(2 p_{2}\right)$. Therefore, Zeeman splitting is sufficient to explain the different spectral width provided by Eqs. (3.43) and (3.45). The $2 p_{2}$ transition spectral width hardly changes, while the spectral width of the $2 p_{4}$ transition is reduced by almost a half.

In addition to Zeeman broadening, these transitions are affected by laser saturation broadening, in which greater laser power does not result in greater signal [159]. This effect manifests as a laser power dependence of the spectral width, as shown in Fig. 3.32. Spectral widths measured with the $2 p_{2}$ and $2 p_{3}$ transitions show strong 

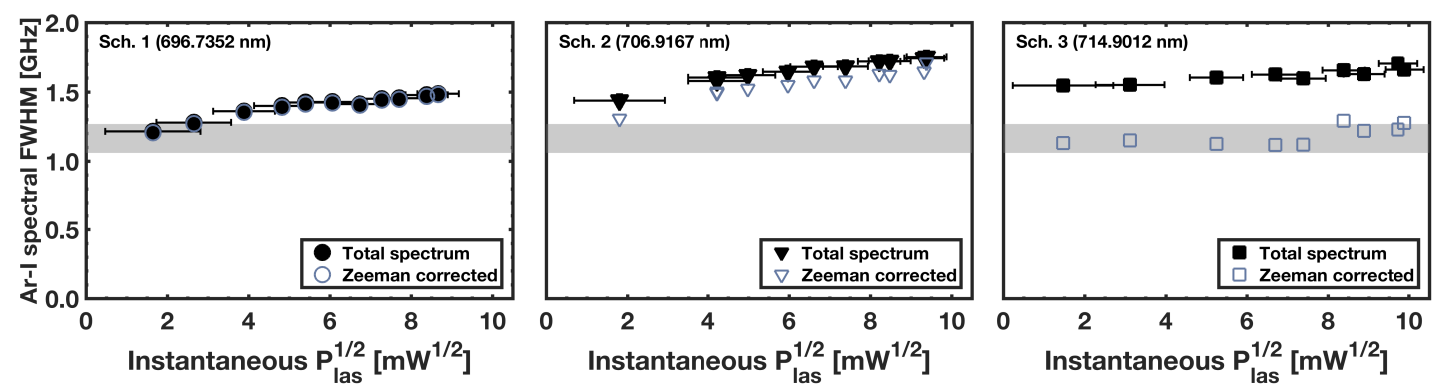

FiguRE 3.32: The spectral width measured by each transition depends on the injected laser power. The $2 p_{2}$ and $2 p_{3}$ transitions are most affected. The $2 p_{4}$ transition is minimally affected by laser saturation, but shows strong Zeeman broadening [25]. (c) 2018 IOP Publishing. Reproduced with permission. All rights reserved.

dependence on laser power. Temperatures recorded with these transitions are not valid for laser powers above approximately $10 \mathrm{~mW}$ in the test plasmas of Fig. 3.32. Since the saturation level is dependent on the density of the probed species, i.e., on the $1 s_{5}$ metastables, best practice is to select a laser power so that the spectral width does not change with increasing laser power.

The $\pi$ transitions are forbidden in the Zeeman splitting of the Ar-I metastable spectrum parallel to the magnetic field. The spectrum splits into two $\sigma_{ \pm}$clusters whose separation depends on the magnetic field. The dependence is linear in $B$. Fig. 3.33 shows an example spectrum with the two $\sigma_{ \pm}$clusters resolved. The three transitions exhibit different dependence of the splitting on the magnetic field, as described by their Landé factors. Still, any of the three transitions can be used to determine the background magnetic field by measuring the separation of the two clusters. The magnetic field dependence of the cluster separation half-distance is shown in Fig. 3.34. This half-distance serves as the shift of the peak away from the drift velocity $u_{n}$ of the neutral population and appears as $\delta \nu_{Z}$ in Eq. (3.43).

\subsubsection{Ar-II LIF}

The experiments reported here measure IVDFs using the scheme proposed by Hill et al. in Ref. [160] and explored in detail by Boivin in Ref. [145]. Fig. 3.35 shows a transition diagram for this sequence. The absorption resonance from the $3 d^{\prime 2} G_{9 / 2}$ 


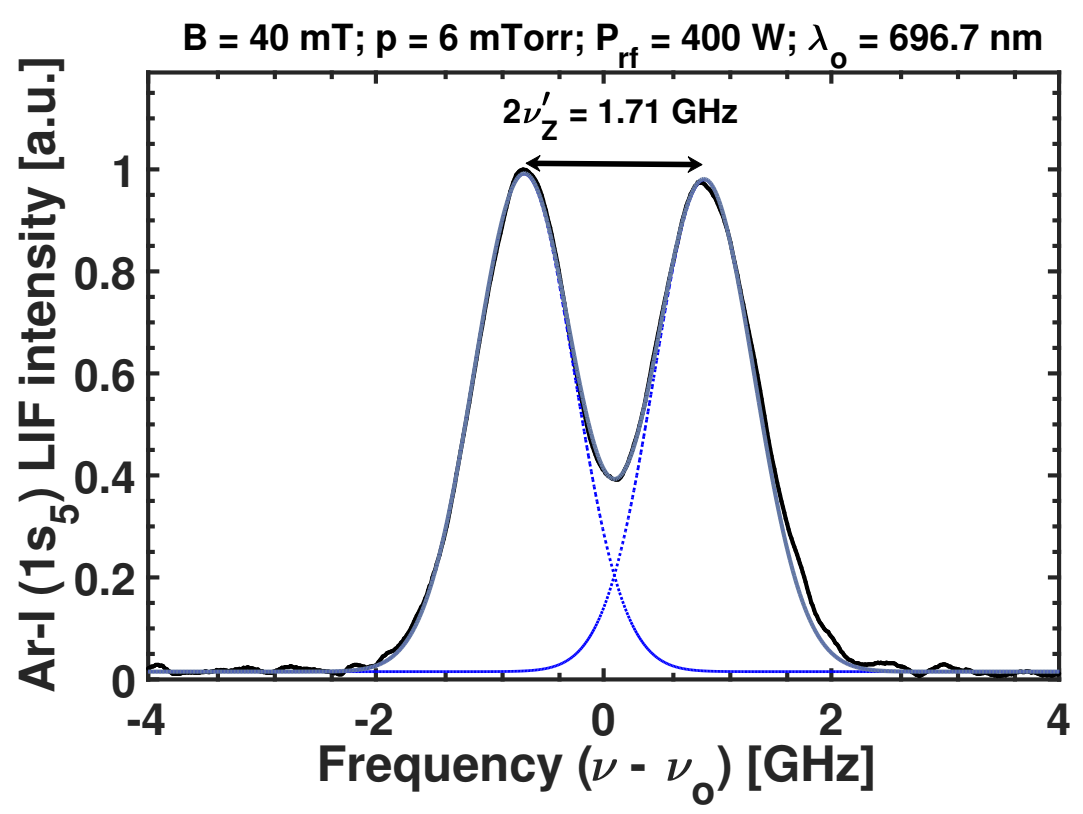

FIgURE 3.33: Ar-I spectrum recorded parallel to the magnetic field, showing $\sigma_{ \pm}$clusters. The separation between the two clusters provides an estimate of the magnetic field strength [25]. (C) 2018 IOP Publishing. Reproduced with permission. All rights reserved.

Ar-II metastable state $\left(\lambda_{\text {vac }}=611.6616 \mathrm{~nm}\right)$ transition to the $4 p^{\prime 2} F_{7 / 2}^{0}$ state is observed through the fluorescence transition to the $4 s^{\prime 2} D_{5 / 2}$ state $\left(\lambda_{\text {vac }}=461.086\right.$ $\mathrm{nm})$. The irreducible velocity uncertainty for this scheme is set by the linewidth of the excitation transition and is reported to be $\Delta u \geq 12 \mathrm{~m} / \mathrm{s}$ [142]. Shot noise and statistical uncertainties typically dominate, with $\Delta u \approx 50 \mathrm{~m} / \mathrm{s}$ common.

\subsubsection{Other considerations}

\section{Reflections}

Occasionally, the LIF laser beam will reflect off a surface in a way that the reflection has sufficient power to create a second peak in the spectrum. This second peak is created by absorption of laser light appearing to arrive from the opposite direction of the primary beam, so that its fluorescence appears to be mirrored on the opposite side of zero velocity from the primary peak. If the beam does not reflect exactly $180^{\circ}$, the fluorescence will be produced by the projection of the reflected light 


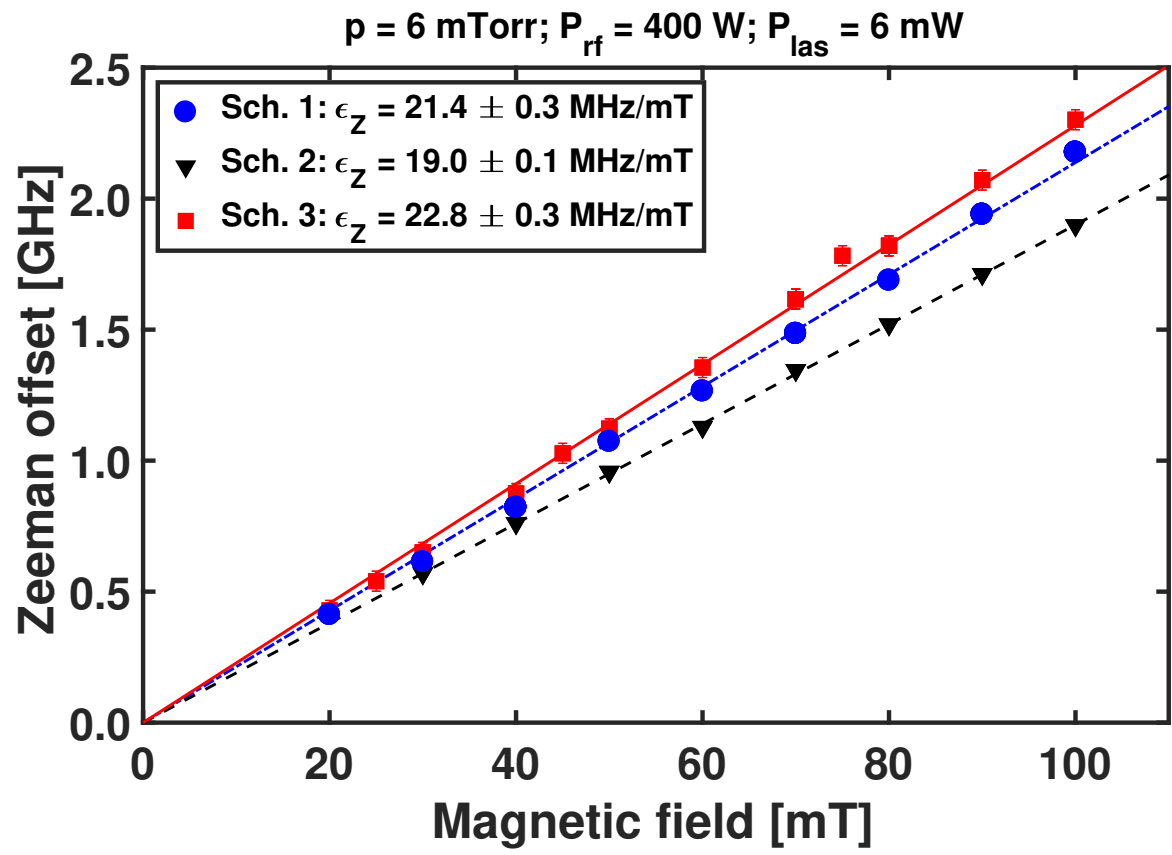

Figure 3.34: Dependence of Zeeman-split cluster separation on $B$ [25]. (C) 2018 IOP Publishing. Reproduced with permission. All rights reserved.

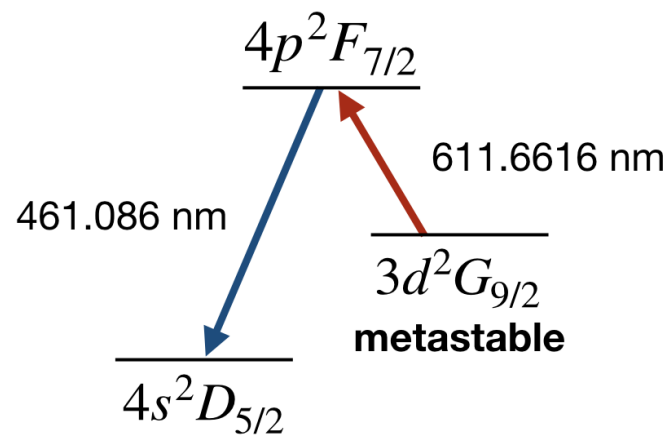

FiguRE 3.35: Transition diagram for metastable Ar-II LIF.

along the primary beam direction. This results in a reflected component that is broader than the primary peak.

Fig. 3.36 shows an example progression of the IVDF as the sample region approaches the boundary from top to bottom in the figure. $\mathrm{d}_{\mathrm{y}}$ is the distance from the sample region to the boundary surface in the vertical direction. The IVDF far from the plate at $\mathrm{d}_{\mathrm{y}}=10 \mathrm{~mm}$ does not have a significant reflection signal. The reflection signal increases as the sample region approaches the boundary surface, becoming comparable in intensity to the IVDF at $\mathrm{d}_{\mathrm{y}}=1 \mathrm{~mm}$. The exception is the measurement at $\mathrm{d}_{\mathrm{y}}=5 \mathrm{~mm}$, where the laser terminates on a razor blade beam dump that strongly attenuates the reflected signal. The functional form for 


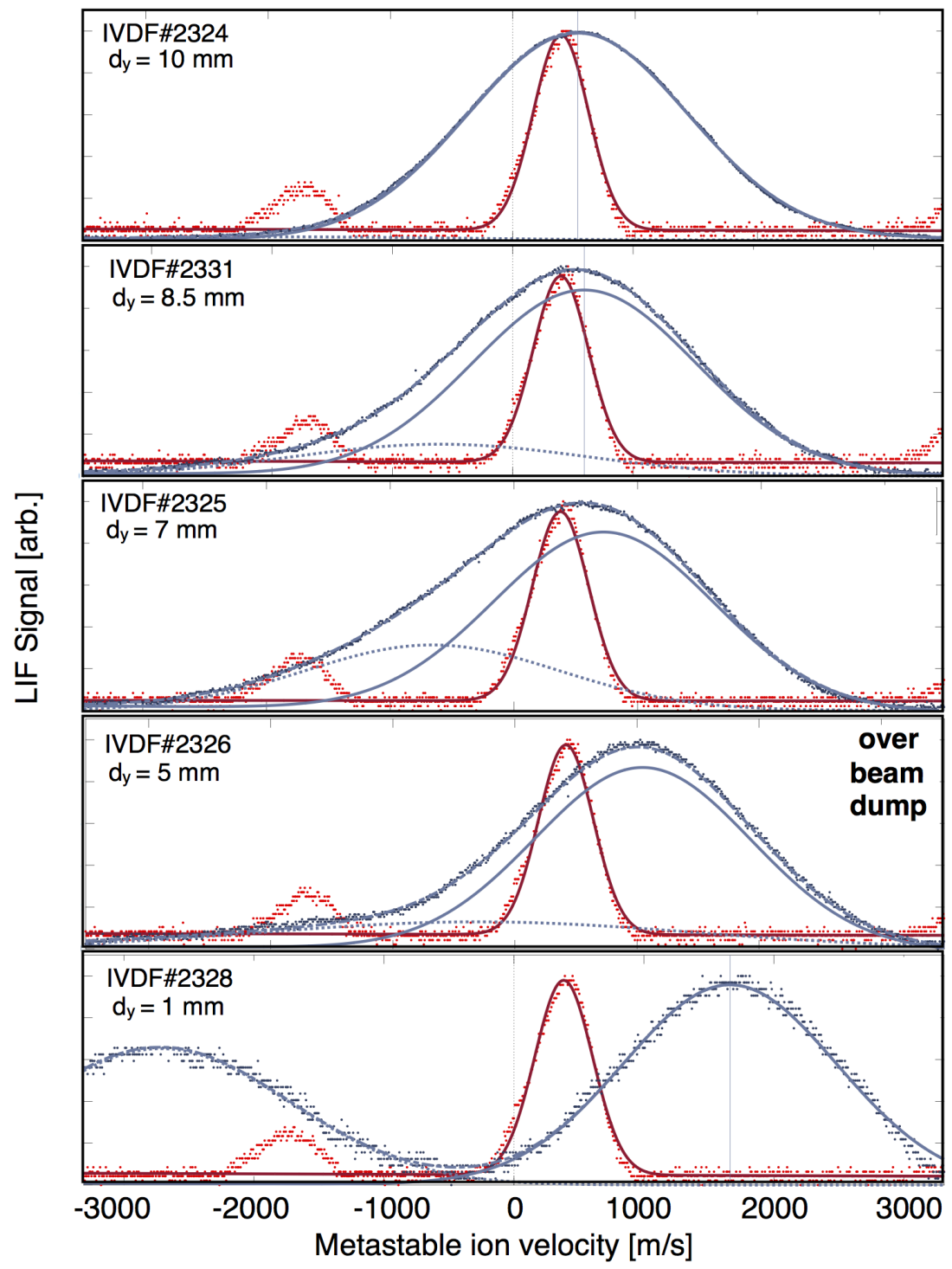

FigURE 3.36: IVDF showing the effect and analysis of laser reflections. Red curves depict reference iodine distribution. Dark blue dots depict argon metastable LIF signal. In light blue: primary IVDF (solid), reflection (dots), combined (dash).

measurements which include reflected signals is a modified version of Eq. (3.44):

$$
I_{\mathrm{LIF}}^{*}(v)=A+C_{\text {inj }} \exp \left[-\left(m / 2 k_{B}\right) \frac{(v-u)^{2}}{T}\right]+C_{\text {refl }} \exp \left[-\left(m / 2 k_{B}\right) \frac{(v+u)^{2}}{D_{\text {refl }}}\right]
$$

$A, C_{\mathrm{inj}}, C_{\mathrm{refl}}, u, T$, and $D_{\text {refl }}$ are fit parameters and serve similar functions to their counterparts in Eq. (3.44). The second Gaussian, which describes the reflected signal, is expected to be centered at the negative velocity of the primary peak. (The width of the reflected peak $D_{\text {refl }}$ is not truly a temperature and is given this 
less suggestive notation.)

\section{Power budget}

The expected final transmission through an optical train is a useful figure of merit when aligning the laser to optical fibers and injection optics. As a general rule, as long as the laser is stable, more power is better as signal scales linearly with power in unsaturated single-photon LIF. If $P_{o}^{\text {las }}$ is the power of the laser as it exits the laser cavity, and $T_{\mathrm{c}}$ is the transmission coefficient of an optical component $c$, the total power injected into the plasma $P^{\text {las }}$ is

$$
P^{\text {las }}=P_{o}^{\text {las }} \prod_{c} T_{c}
$$

A list of the optical components and their transmission coefficients is shown in Tab. 3.8. The mechanical chopper transmits with $50 \%$ cycle-averaged transmission, so that a power reading downstream is observed to have half the power when the mechanical chopper is rotating. The plasma, however, is exposed to instantaneous laser power oscillating from $0 \%$ to full transmission.

As an example calculation, the Ar-II laser passes through the splitter, the optical diode, across two mirrors, through a multimode fiber, reflects off another mirror, through the linear polarizer, through a lens, and finally through the chamber window. If the initial power is $1300 \mathrm{~mW}$, the transmitted power is

$$
\begin{aligned}
P^{\text {las }} & =(0.90 \times 0.80 \times 0.95 \times 0.95 \times 0.80 \times 0.95 \times 0.80 \times 0.95 \times 0.95) \times 1300 \mathrm{~mW} \\
& =464 \mathrm{~mW}
\end{aligned}
$$

This constitutes a 36\% overall transmission for a multimode fiber. A similar calculation for a single-mode fiber with the linear polarizer, $\lambda / 4$-wave plate combination gives an expected transmitted power of $114 \mathrm{~mW}$, or $9 \%$ total transmittance. To excellent approximation, these transmittances are constant over laser power and can be applied directly to the exit power of the laser to determine the power expected at the final output of the injection optics. In this way the laser power can 


\begin{tabular}{lc}
\hline \hline Component & Transmission coefficent, $T_{c}$ \\
\hline 90:10 beam splitter & 0.90 \\
Optical diode & 0.80 \\
Each lens or mirror & 0.95 \\
MM fiber & 0.80 \\
SM fiber & 0.25 \\
Linear polarizer & 0.80 \\
Lin. pol. $+\lambda / 4$-plate & 0.60 \\
Clean chamber window & 0.95 \\
\hline \hline
\end{tabular}

TABLE 3.8: Approximate transmission coefficients of elements in the LIF optical train.
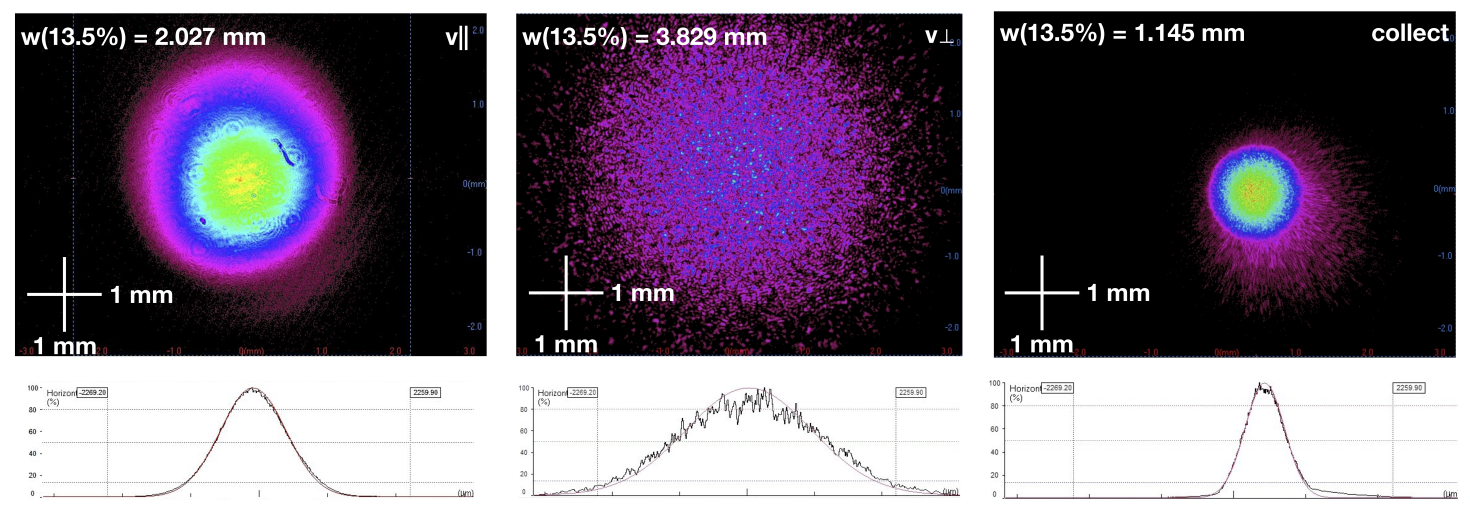

FigURE 3.37: Beam profiles for parallel and perpendicular LIF. LIF measurements record an intensity-weighted average over the beam spot. Beam profiler records images with a $9.3 \mu \mathrm{m} /$ pixel sensor. Only horizontal profiles and widths are shown.

be checked overall or observed after each optical component to reduce any sources of power loss to a minimum.

\section{Laser beam profiles}

The collimators used for LIF injection create a Gaussian profile beam. The axial injection beam diameter is smaller than the beam diameter for perpendicular injection, as detailed in Sec. 3.3.4. The intensity distributions in the profiles in Fig. 3.37 reveal the structure of the injected beam and the weighting of the emission due to the collection optics. These intensity distributions are important, because 


\begin{tabular}{lcc}
\hline \hline Response function & $w\left(13.5 \%, 1 / e^{2}\right)[\mathrm{mm}]$ & $\sigma[\mathrm{mm}]$ \\
\hline Laser, $\perp$ & 3.829 & 0.957 \\
Horizontal & 3.934 & 0.258 \\
Vertical & & \\
& & \\
\hline Laser, $\|$ & 2.027 & 0.507 \\
Horizontal & 2.192 & 0.548 \\
Vertical & & \\
& & \\
Collection focus $(\perp$ and $\|)$ & 1.145 & 0.286 \\
Horizontal & 1.198 & 0.300 \\
Vertical
\end{tabular}

TABLE 3.9: Response function parameters measured by beam profiles of the injected laser and backlit collection optics.

the signal that is recorded depends not only on the state of the plasma but also on the structure of the injection beam and the collection region.

The emission collected by the PMT, $f_{\mathrm{pmt}}(\mathbf{x}, \mathbf{v})$, due to the ideal distribution $f(\mathbf{x}, \mathbf{v})$ and the optical response function $\mathscr{P}\left(\mathbf{x} ; \mathbf{x}^{\prime}\right)$ is

$$
f_{\mathrm{pmt}}(\mathbf{x}, \mathbf{v})=\int f\left(\mathbf{x}^{\prime}, \mathbf{v}\right) \mathscr{P}\left(\mathbf{x} ; \mathbf{x}^{\prime}\right) d^{3} \mathbf{x}^{\prime}
$$

The optical response function $\mathscr{P}\left(\mathbf{x} ; \mathbf{x}^{\prime}\right)$ is the product of the laser and collection optics response functions: $\mathscr{P}\left(\mathbf{x} ; \mathbf{x}^{\prime}\right)=\mathscr{P}_{\text {las }}\left(\mathbf{x} ; \mathbf{x}^{\prime}\right) \mathscr{P}_{\text {coll }}\left(\mathbf{x} ; \mathbf{x}^{\prime}\right)$. Unless they are observed to be otherwise, the response functions are given a Gaussian form:

$$
\begin{aligned}
& \mathscr{P}_{\text {las }}\left(\mathbf{x} ; \mathbf{x}^{\prime}\right)=\mathscr{P}_{l, o} \exp \left[-\frac{\left(\mathbf{x}^{\prime}\right)^{2}}{2 \sigma_{l}^{2}}\right] \\
& \mathscr{P}_{\text {coll }}\left(\mathbf{x} ; \mathbf{x}^{\prime}\right)=\mathscr{P}_{c, o} \exp \left[-\frac{\left(\mathbf{x}^{\prime}\right)^{2}}{2 \sigma_{c}^{2}}\right]
\end{aligned}
$$

The $13.5 \%$ level $I / I_{o}$ normalized intensity widths are given in Fig. 3.37. $\sigma_{l}$ and $\sigma_{c}$ are calculated from fitting Eqs. (3.49) to the beam profiles. The results are shown in Tab. 3.9. The calculated response functions are used in creating synthetic 
diagnostics for comparing simulations to measurement, and simulated spectra are compared to the normalized spectra $f_{\text {pmt }} / \mathscr{P}_{c, o} \mathscr{P}_{l, o}$.

One implication of these finite extent response functions is that gradients with shorter scales than the overlap region will be misrepresented by the LIF data. Boundary regions, and Debye sheaths especially, have steep gradients over tens of microns, and are far too small to be resolved by LIF on HELIX. LIF measurements that overlap these regions therefore record an ensemble of sheath particles combined with particles outside the sheath, and the measured gradients appear smoothed. 


\section{Chapter 4}

\section{Three-dimensional, multispecies}

\section{measurements in a boundary with}

an oblique magnetic field: $B=600$

$\mathrm{G}, \psi=74^{\circ}$

\subsection{Introduction}

Measurements were made of the ion and neutral velocity distribution functions $(\mathrm{I} / \mathrm{NVDF})$ and the electron energy probability functions (EEPFs) in three dimensions in a plasma boundary with magnetic field magnitude $B=600 \mathrm{G}$ and magnetic angle $\psi=74^{\circ}$. The introduction of the absorbing boundary caused the plasma to reestablish an equilibrium that is shifted from the mechanical axis by several centimeters, as shown in Fig. 4.1. Statements like "outer radii" refer to plasma radius, not mechanical radius. Unless stated otherwise, data are presented in the $(x, y, z)$ simulation coordinate system, and $z=0 \mathrm{~mm}$ lies along the mechanical axis. When the $\left(x^{*}, y^{*}, z^{*}=z\right)$ laboratory coordinate system is used it will be indicated. 


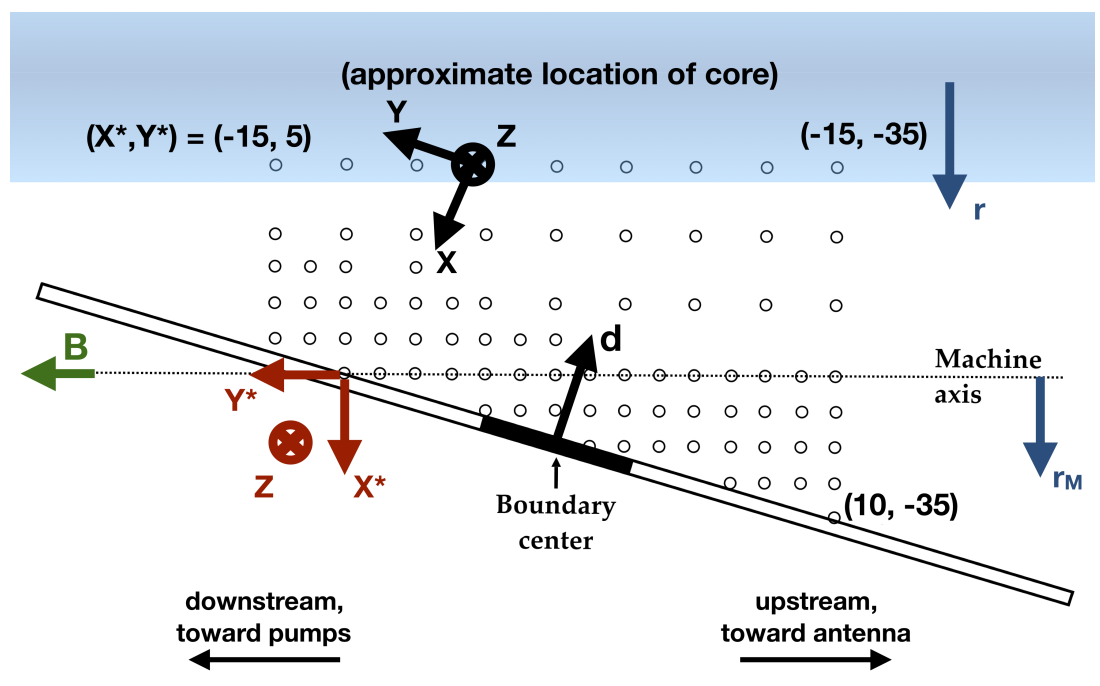

FIgURE 4.1: Detail of the coordinate systems showing the plasma shift. The $\left(x^{*}, y^{*}, z^{*}\right)$ system is aligned to the chamber mechanical axis and the point where it intersects the boundary surface. The un-starred system is aligned with the boundary geometry, and $z=z^{*}$, with the two used interchangeably. The four corners of the experimental domain are provided in the $\left(x^{*}, y^{*}, z^{*}\right)$ system. The mechanical axis lies along $z=z^{*}=0 \mathrm{~mm}$. The distance to the surface $d$ is not necessarily from the boundary center.

\subsection{Magnetic field measurements}

Hall effect and Langmuir probes provide measurements of the background environment for the ion and neutral dynamics in the boundary region. The magnetic field measurements, performed at atmosphere, with the stainless steel boundary in place and aligned, are shown in Fig. 4.2. The magnetic field deviation from the mean does not exceed $\pm 1 \%$ within the experimental domain, with all measured axial fields falling within the range $B_{y^{*}} \in[586,597] \mathrm{G}$. The fields in the two directions perpendicular to the chamber axis are within $B_{z} \in[5,20] \mathrm{G}$ and $B_{x^{*}} \in$ $[25,36] \mathrm{G}(\lesssim 6 \%)$ and are neglected.

In tandem with the boundary in/boundary out measurements shown in Fig. 2.7, these measurements confirm that a) the boundary does not distort the magnetic field structure by more than $1 \%$ and b) the magnetic field is axial and within $1 \%$ of $\bar{B}=591 \mathrm{G}$ throughout the entire experimental domain. To within very good approximation, the magnetic field is linear, axial, uniform, and incident on the boundary at $\psi=74^{\circ}$. While care was taken to align the gaussmeter probe for 
this measurement, the stalk of the probe is not rigid, and small angle variations in orientation likely account for a large component of the remaining spread in the magnitudes reported. The remainder is spatial variation from discrete electromagnets. The structure apparent in Fig. 4.2, where the field magnitude is highest at the top corners and lowest at the lower center of domain, results from the spacing of the electromagnets, but these variations are small.

\subsection{EEPF, $n_{e}, T_{\text {eff }}$, and $p_{e}$ from Langmuir probe measurements}

The plasma electrons are assumed to be magnetized and follow the field lines. (This assumption will be verified in Sec. 4.6.) Druyvesteyn analysis (see Sec. 3.2.3.3) was employed throughout, because it does not require that the electrons possess a Maxwellian energy distribution, and because it gave close results to the full, extended Druyvesteyn analysis in significantly less time. As discussed in Sec. 3.2.3.3, the relative electron, ion, and probe scales produce a density that is underestimated $(\approx 20 \%)$, and a temperature that is slightly overestimated $(\approx 10 \%)$, when Druyvesteyn analysis is employed.

\subsubsection{Non-Maxwellian features are observed in EEPFs}

The electron energy probability function (EEPF) is constructed from the second derivative of the electron current. Fig. 4.3 shows examples of typical EEPFs observed in the Langmuir probe data. The $0^{\text {th }}$ and $1^{\text {st }}$ moments of these EEPFs provide density and electron temperature, respectively (see Sec. 3.2.3.3). NonMaxwellian electron populations appear in the EEPFs. For example, the EEPF in (a) contains an energetic electron distribution with a peak at approximately 18 $\mathrm{eV}$. These EEPFs, plotted on the same scale, indicate a radial density gradient directed toward $-\hat{x}^{*}$. That the gradient is not characterized by proximity to the boundary surface is seen in part (b), which shows measurements at constant $x^{*}$ 
$Z=-10 \mathrm{~mm} X Y$ plane

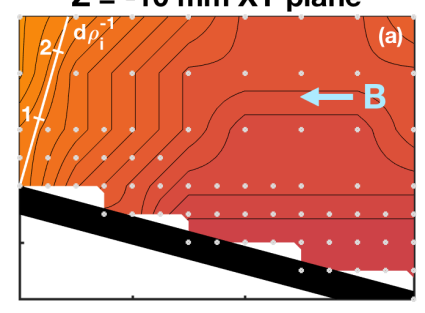

$Z=-5 \mathrm{~mm} X Y$ plane
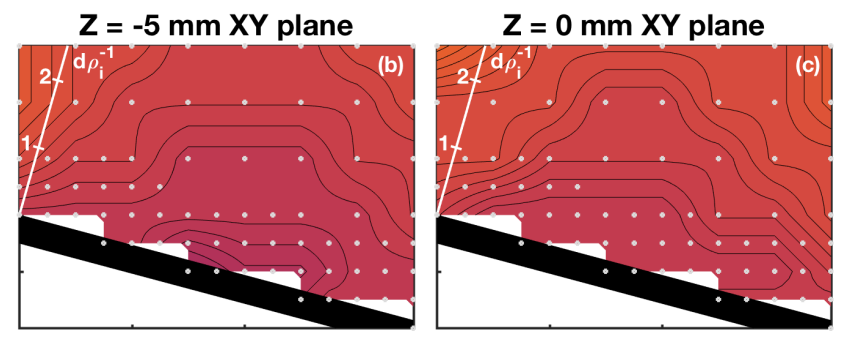

$Z=+5 \mathrm{~mm} X Y$ plane

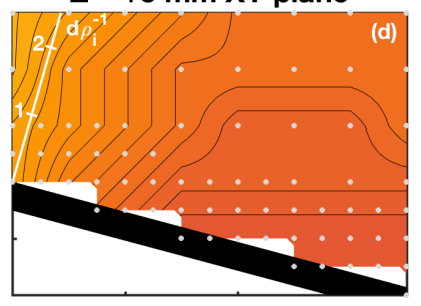

$Z=+10 \mathrm{~mm} X Y$ plane

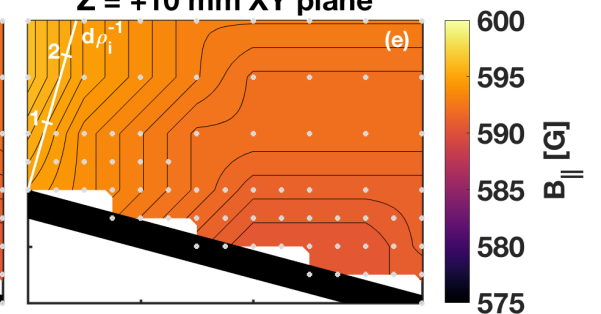

Figure 4.2: Percentage spatial variation in magnetic field: $\bar{B}_{\|}=\bar{B}_{y^{*}}=591 \mathrm{G}$, with the field direction indicated in light blue in (a). The magnetic field deviation from the mean does not exceed $\pm 1 \%$ within the experimental domain. The solid black bar across the bottom marks the location of the surface. White dots mark the measurement locations. White regions otherwise mark regions where no measurements were taken. These regions are slightly accentuated in the near vicinity of the surface. The plate normal distance $d$ is shown normalized to the ion gyro-radius $\rho_{i}$. 

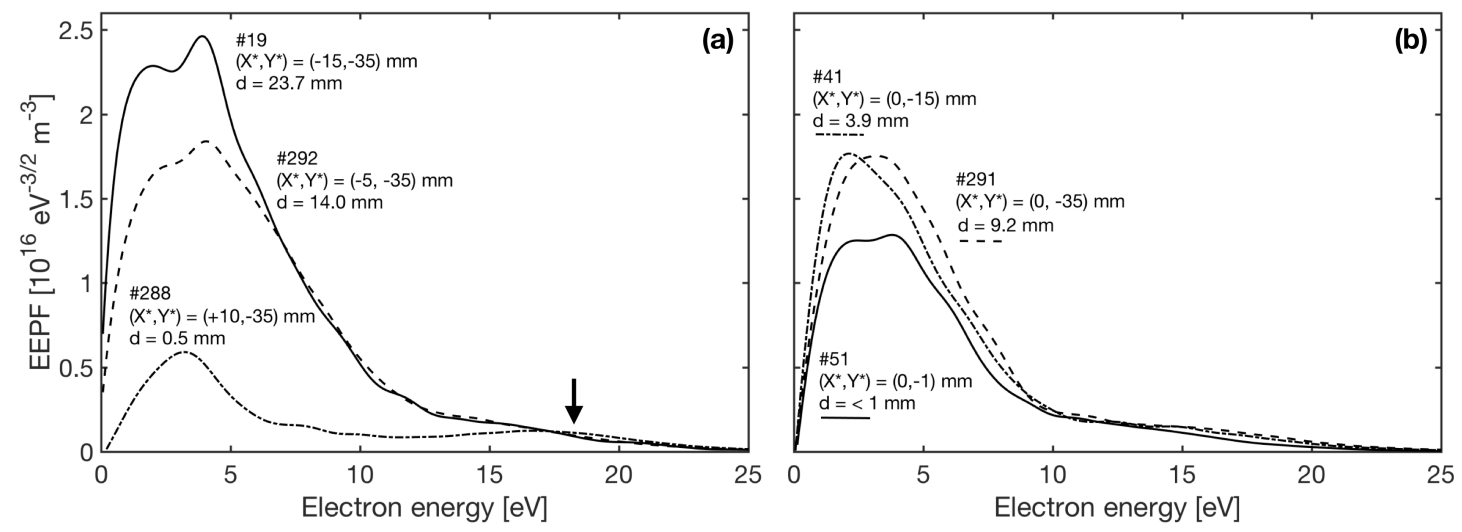

FIgURE 4.3: EEPF dependence on plasma (a) radius and (b) axial location. In (a), the arrow marks the presence of an energetic population at large radius.

but at several distances from the surface. At all three of these locations the EEPFs contain non-Maxwellian, enhanced high-energy tails.

The first excited state of argon is $11.55 \mathrm{eV}$, and the first two ionization energies are $15.76 \mathrm{eV}$ and $27.63 \mathrm{eV}$ [161]. The bulk of the electrons in the EEPFs measured in the boundary possess less energy than the first excitation energy; the tail populations are responsible for ionization and excitation to states probed by laser-induced fluorescence (LIF).

\subsubsection{The helicon source creates a radial density gradient}

For much of the boundary region, the electron population is not Maxwellian, and the energy moment formulation of the electron temperature is preferable. Langmuir probe measurements of the plasma density are shown in 3D in Fig. 4.4. Several features emerge from these measurements. First, the plasma density has a distinctly radial structure, with the maximum densities observed at the top of the domain, near the brightest emission regions. The minima are consistently observed at the largest radii in the domain. The range of densities observed is approximately $8 \times 10^{16} \mathrm{~m}^{-3}$ to $7 \times 10^{17} \mathrm{~m}^{-3}$. The plasma density decreases with distance to the boundary surface, but the gradient in this direction is less pronounced than the gradient along the plasma radius. There may be a slight axial density gradient as 

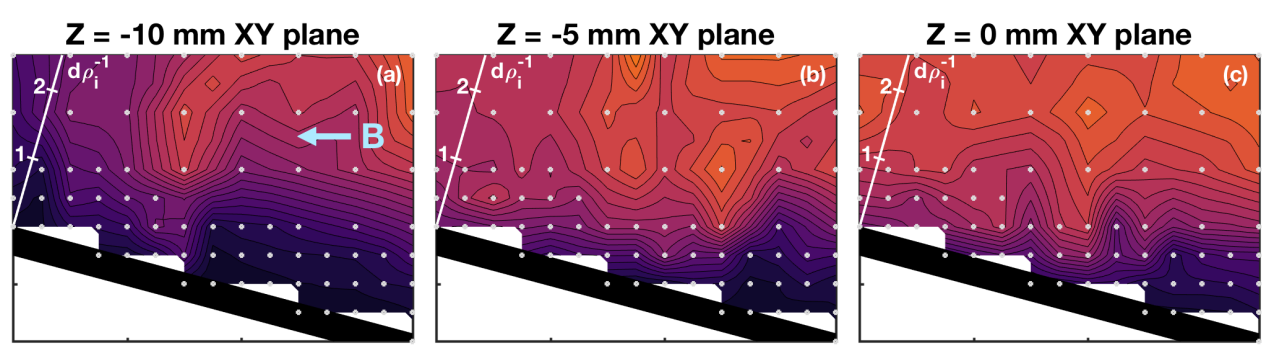

$\mathrm{Z}=+\mathbf{5} \mathrm{mm} X Y$ plane

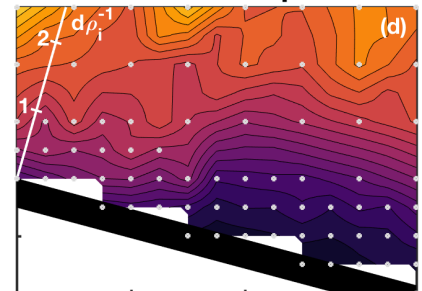

$Z=+10 \mathrm{~mm} X Y$ plane

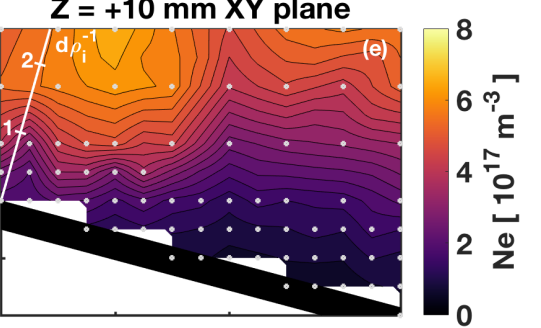

FiguRE $4.4: n_{e}(\mathbf{x})$ in the boundary region. 

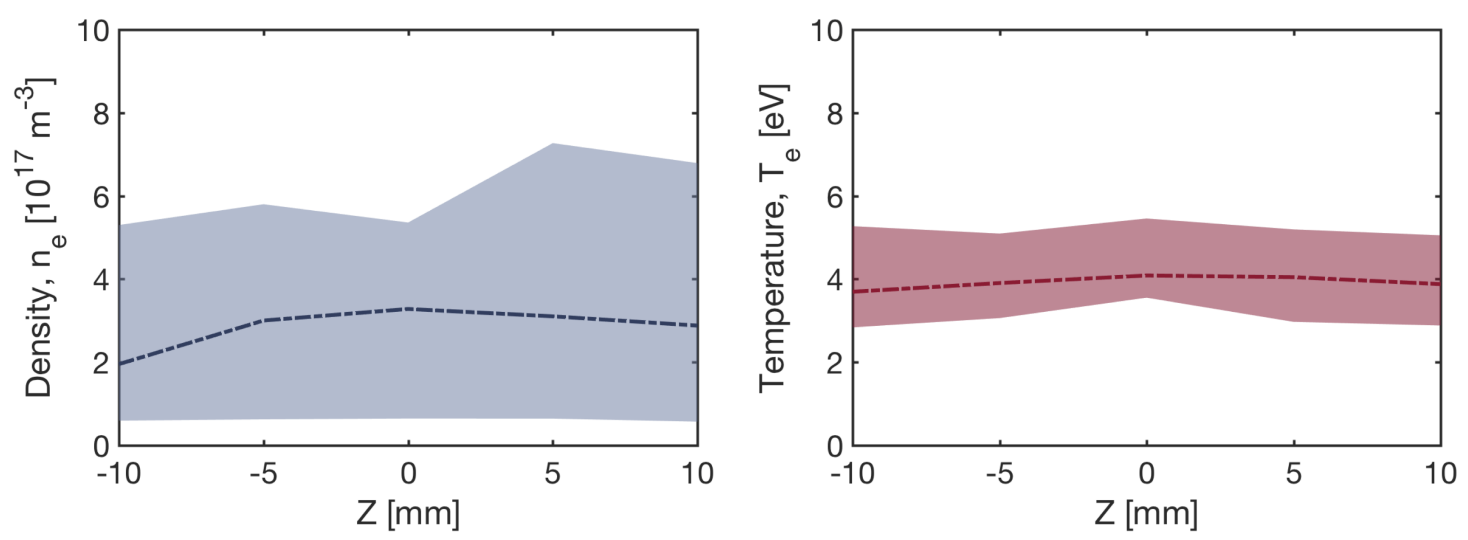

FiguRE 4.5: Electron density and temperature dependence on $z$. Uncertainties are $\delta z= \pm 1 \mathrm{~mm}, \delta n_{e}= \pm 20 \%$, and $\delta T_{\text {eff }}= \pm 10 \%$. The dashed line indicates the mean, and the shaded region indicates the range.

well, directed toward the antenna $\left(-y^{*}\right)$. The approximately $15 \%-20 \%$ decrease in density with increasing $y^{*}$ is on the order of the measurement uncertainty, which is estimated to be $20 \%$, optimistically, or $50 \%$, conservatively. The left plot of Fig. 4.5 shows the mean and peak density in each $z$ plane. The mean density suggests a centrally peaked profile. There is a clear trend toward higher peak density at increasing $z$, suggesting that the plasma core may be shifted a few millimeters in $z$ from the chamber mechanical axis. The experimental domain rests within the more gradual density gradient region slightly away from the high density core.

\subsection{3 $T_{\text {eff }}$ distribution reflects high-energy electron popula- tions}

The electron temperatures are observed to be fairly uniform, especially at $z=0$ $\mathrm{mm}$, the center of the domain. Fig. 4.6 shows a heatmap of the effective electron temperature $T_{\text {eff }}=2\langle\varepsilon\rangle / 3$ distribution in the boundary region.

Two features emerge. First, the temperature at $z=0 \mathrm{~mm}$ is slightly higher than at larger $|z|$. This dependence can be seen in the $T_{\text {eff }}$ mean and range shown in the right plot of Fig. 4.5. $T_{\text {eff }}$ decreases with the distance of each of these planes to the plasma core and is consistent with the cylindrical structure of the 

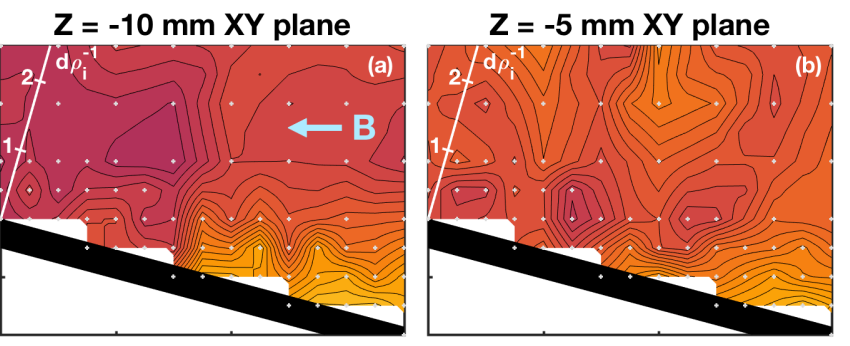

$Z=0 \mathrm{~mm} X Y$ plane

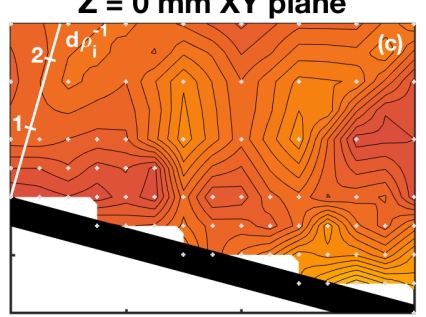

$Z=+5 \mathrm{~mm} X Y$ plane

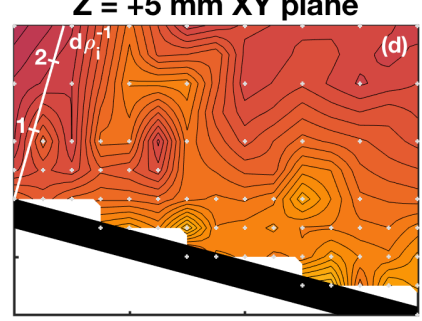

$Z=+10 \mathrm{~mm} X Y$ plane

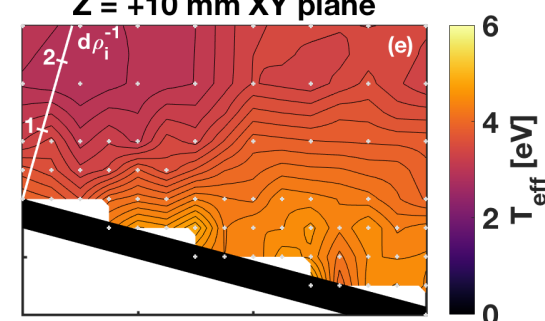

FIGURE 4.6: $T_{\text {eff }}(\mathbf{x})$ in the boundary region. 
helicon plasma. The second feature that arises is the spatial distribution of the nonMaxwellian features of the EEPFs shown in Fig. 4.3. The high-energy content of the EEPFs increases with plasma radius as the density decreases, and the resulting high effective temperature appears at the outermost radii $\left(x^{*} \gtrsim 5 \mathrm{~mm}\right)$ of the experimental domain in Fig. 4.6.

\subsection{4 $p_{e}=n_{e} k_{\mathrm{B}} T_{\mathrm{e}}$ shows a radial helicon electron pressure gradient}

The spatially varying density and relatively uniform electron temperature lead to an electron pressure, calculated by the ideal gas law, $p_{e}=n_{e} k_{\mathrm{B}} T_{\mathrm{e}}-n_{e}$ is the electron density, $k_{\mathrm{B}}$ is the Boltzmann constant, and $T_{e}=T_{\text {eff }}$ is understood that is largely determined by the structure of the density, as shown in Figs. 4.7 and 4.8. The cylindrical geometry of the helicon plasma is evident in the radial dependence of $p_{e}$ in these images, with the pressure decreasing for increasing $x^{*}$. $p_{e}$ decreases at larger $|z|$ because those planes are slightly farther from the high density core.

\section{4 $\phi_{p}$ depends on proximity to the boundary sur- face}

The plasma potential $\phi_{p}$ from Langmuir probe analysis is unreliable due to the asymmetry of the system caused by the magnetic field and because the probe disturbs the presheath $[3,27]$. The second derivative of the probe current provides a guideline for $\phi_{p}$. However, this technique is expected to underestimate the actual potential by $\mathcal{O}\left(10^{-1} T_{\mathrm{e}}\right)$ (see Sec. 3.2.3.3). $\phi_{p}$ is shown in Fig. 4.9. Unlike $n_{e}$ and $T_{e}, \phi_{p}$ is organized in relation to the distance from the boundary surface, instead of in relation to plasma radius. This behavior has been observed in unmagnetized sheaths at absorbing boundaries [3] and is not surprising here. Variations across z 


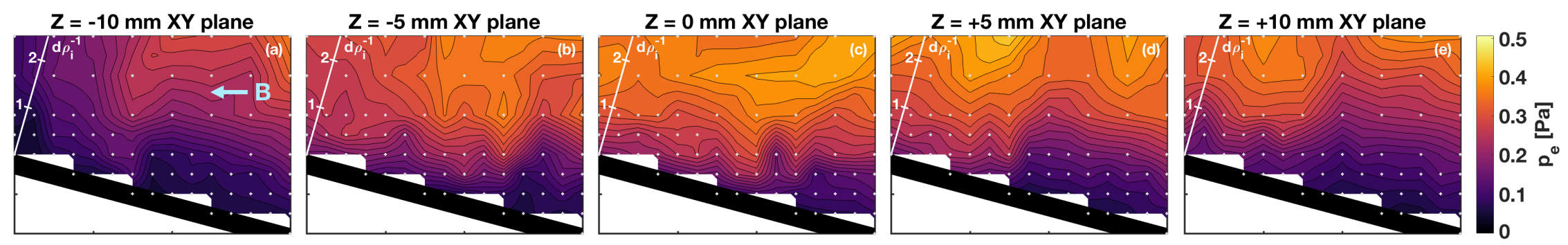

FigURE 4.7: Spatial structure of the electron pressure, $p_{e}$. 

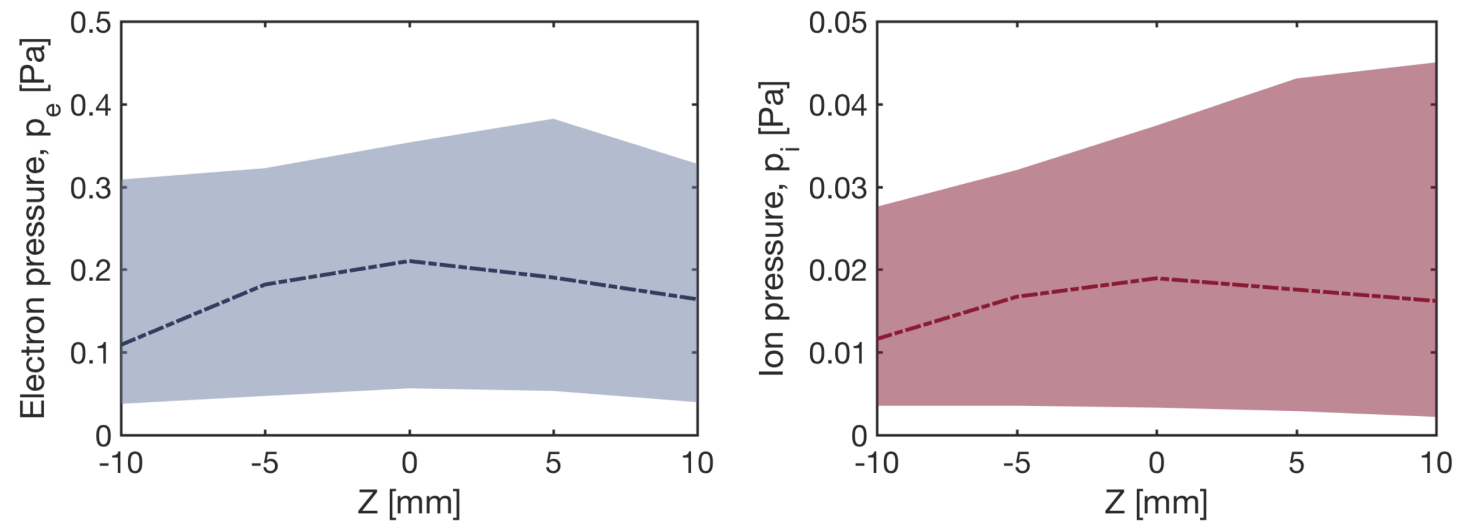

Figure 4.8: Electron and ion pressure dependence on $z$. The dashed line indicates the mean and the shaded region indicates the range. 
$Z=-10 \mathrm{~mm} X Y$ plane
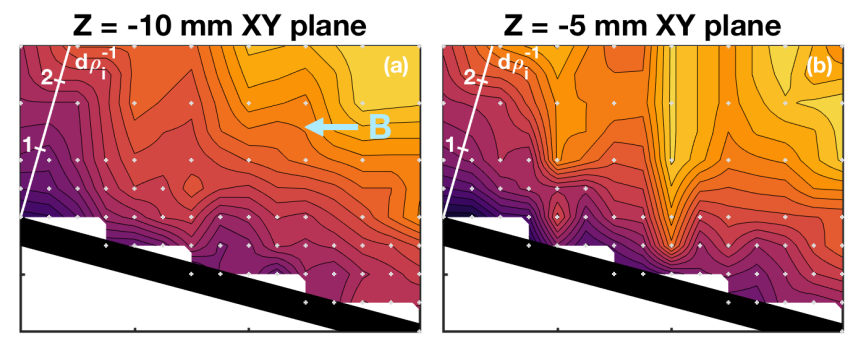

\section{$\mathbf{Z}=\mathbf{0} \mathrm{mm} X Y$ plane}

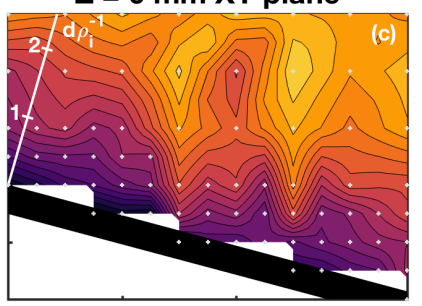

$\mathrm{Z}=+5 \mathrm{~mm} X Y$ plane

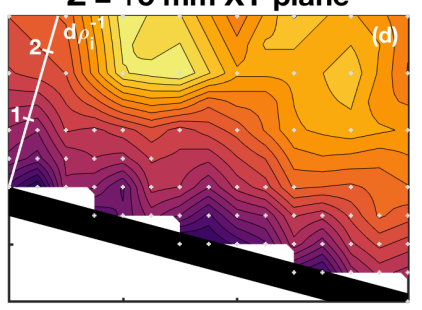

$Z=+10 \mathrm{~mm} \mathrm{XY}$ plane

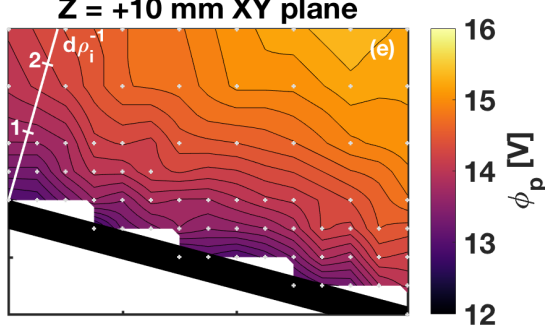

FiguRE 4.9: $\phi_{p}(\mathbf{x})$ in the boundary region. 
are within the measurement uncertainty of $\delta \phi_{p} \approx 0.5 \mathrm{~V}$. The electric field associated with this gradient accelerates the ions toward the boundary surface, diverting them from their trajectories along the field lines. This field is also responsible for generating any $\mathbf{E} \times \mathbf{B}$ drifts along the boundary.

\subsection{Ion temperature and pressure are observed in three dimensions from LIF distributions}

\subsection{1 $T_{i}$ is slightly anisotropic}

The ion temperature $T_{i}$ is measured from the width of the LIF spectrum, as described in Sec. 3.3.2. LIF resolves the ion temperature $T_{i}$ separately in every injection direction. In this instance $T_{i, y^{*}}=T_{i, \|}$, and $T_{i, x^{*}}$ and $T_{i, z}$ are perpendicular to the applied field. The mean temperature over all three directions is $0.35 \mathrm{eV}$ with a standard deviation of $0.05 \mathrm{eV} . T_{i}(\mathbf{x})$ is shown in Fig. 4.10, each row of which shows the temperature along one lab-frame direction.

$T_{i, x^{*}}$ increases by $\leq 0.05 \mathrm{eV}$ from the upstream to the downstream edge of the domain. The ions in this direction cool slightly just above the surface. At $z<0$ $\mathrm{mm}$, there is a thin layer of ion heating where temperatures $0.09 \mathrm{eV}$ above the mean are observed just above the cool region at the boundary surface. Measurements in this direction suffer from distortion due to laser reflections of the boundary surface. A circular laser beam dump at the center of the boundary, marked by blue patches in (b)-(d) and (g)-(i), mark the extent of the laser beam dump in each plane. This beam dump substantially, but not entirely, reduces the amount of reflected signal in the LIF spectrum (see "Reflections" in Sec. 3.3.8). The drop in temperature may be a result of an inaccurate reflected signal fitting algorithm. It is significant that the measurements taken when the laser is incident on the beam dump show temperatures that are comparable to the measurements when the laser is not incident on the beam dump. The same fitting algorithm - identify the LIF signal in the injection direction with mean flow $u$, look for a second peak at $-u$ 

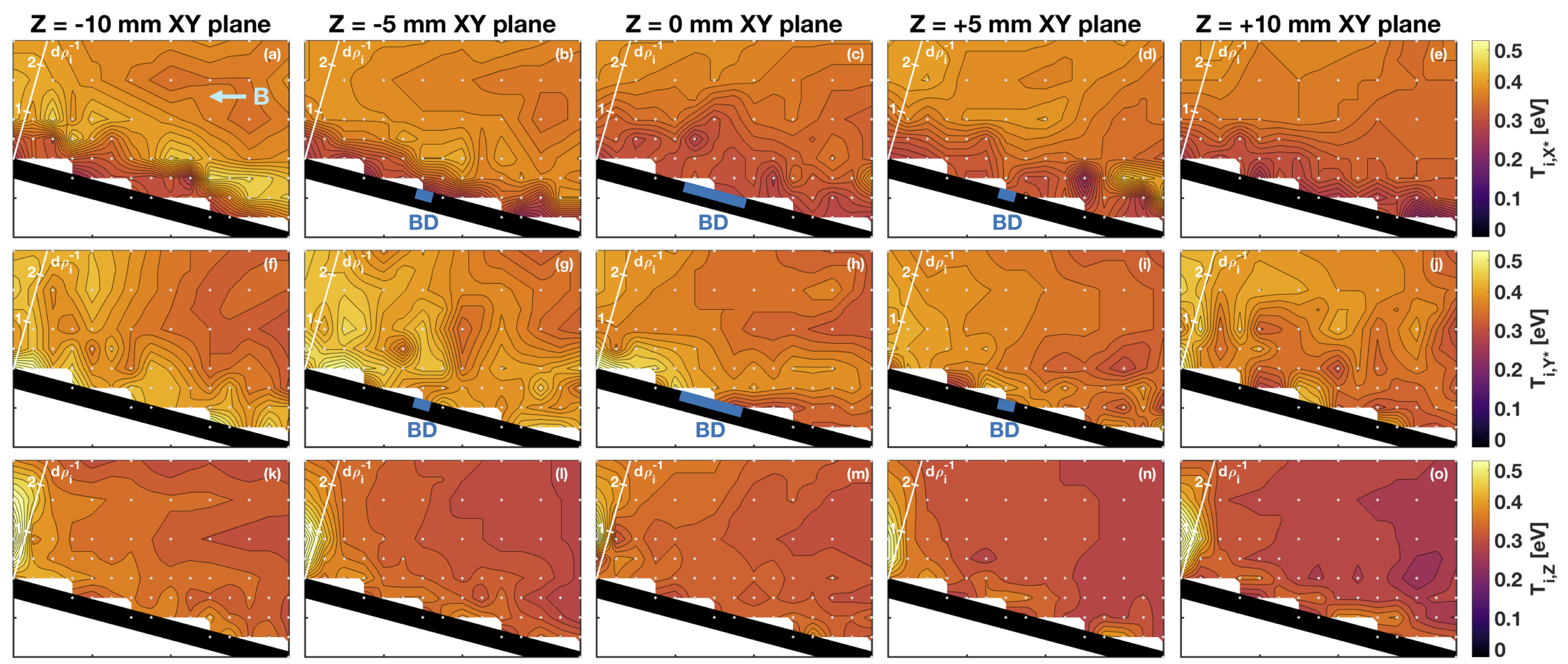

FIGURE 4.10: $T_{i}(\mathbf{x})$ in 3D3V. $T_{i, x^{*}}$, shown in the top row in (a)-(e), represents one of the temperature components perpendicular to the magnetic field. The temperature parallel to the magnetic field, $T_{i, y^{*}}=T_{i, \|}$, is shown in (f)-(j). The second perpendicular direction, $T_{i, z^{*}}$, is shown in the bottom row in (k)-(o). As before, each column represents one planar location in $z$. The hot spot on the downstream (left) side of (k)-(o) is an artifact from laser reflections. Blue patches in (b)-(d) and (g)-(i) mark the extent of the laser beam dump (BD) in each plane. For the measurements in $(\mathrm{k})-(\mathrm{o})$, the laser passes across the boundary surface and does not contact the beam dump. 
but with arbitrary temperature, then fit for $u$ and $T_{i}$ - is applied over the entire domain. Far from the surface, this algorithm finds a reflected signal contribution $<1 \%$.

$T_{i, z}$ increases from $0.26 \mathrm{eV}$, at the upstream entrance to the domain on the right side of (k)-(o) of Fig. 4.10, to approximately $0.40 \mathrm{eV}$ near the wall downstream (left). There is an axial gradient apparent in the data, with a slight increase in $T_{i, z}$ just above the surface. The hot spot on the downstream side of (k)-(o) is an artifact from laser reflections and does not represent the true temperature of the ions there.

Parallel to the magnetic field, $T_{i, y^{*}}$ increases approximately $0.05 \mathrm{eV}$ between the upstream and downstream edges of the domain. Temperature gradients with respect to the distance from the surface are inconsistent across $z$. Temperatures in this direction are slightly higher, with mean temperature $\bar{T}_{i, y^{*}}=0.39 \mathrm{eV}$, compared to $\bar{T}_{i, x^{*}}=0.36 \mathrm{eV}$ and $\bar{T}_{i, z}=0.33 \mathrm{eV}$. The temperature measurement uncertainty $\delta T_{i}$ arises from systematic errors in the technique and from statistical variation in identical measurements. $\delta T_{i}$ (syst.) $= \pm 0.012 \mathrm{eV}$, and the statistical uncertainties calculated from fiducial measurements are shown in Tab. 4.1. The temperature anisotropy is therefore greater than the uncertainty in the measurement. A higher axial temperature is consistent with the presumed greater diffusivity of ions along the field lines compared to cross-field transport. Preferential acceleration along the boundary electric field and subsequent thermalization causes the disparity that $\bar{T}_{i, x^{*}} \neq \bar{T}_{i, z}$, even though these temperatures both describe the motion of particles across field lines.

\subsubsection{The ion pressure is isotropic}

The ion temperatures in Fig. 4.10 are used to construct the ion pressure in 3D3V, which is shown in Fig. 4.11. In this figure, the directional ion pressures are calculated from $p_{i, x^{*}}=n_{i} k_{\mathrm{B}} T_{i, x^{*}}, p_{i, y^{*}}=n_{i} k_{\mathrm{B}} T_{i, y^{*}}$, and $p_{i, z^{*}}=n_{i} k_{\mathrm{B}} T_{i, z^{*}}$. The pressures are remarkably similar in all three directions and are determined primarily 


\begin{tabular}{lll}
\hline \hline Component & $\delta T_{i}[\mathrm{eV}]$ & IVDFs contributing, \# \\
\hline$T_{i, x^{*}}$ & 0.010 & $2291-2295$ \\
$T_{i, y^{*}}$ & 0.011 & $2743-2747$ \\
$T_{i, z}$ & 0.015 & $3297-3300,3396,3397,3575$ \\
& & $3576,3666,3667,3760,3761$ \\
\hline \hline
\end{tabular}

TABLE 4.1: Statistical uncertainty $\delta T_{i}$ (syst.) calculated via repeated fiducial measurements. $\mathbf{x}^{*}=(-10,-30,0) \mathrm{mm}$. 

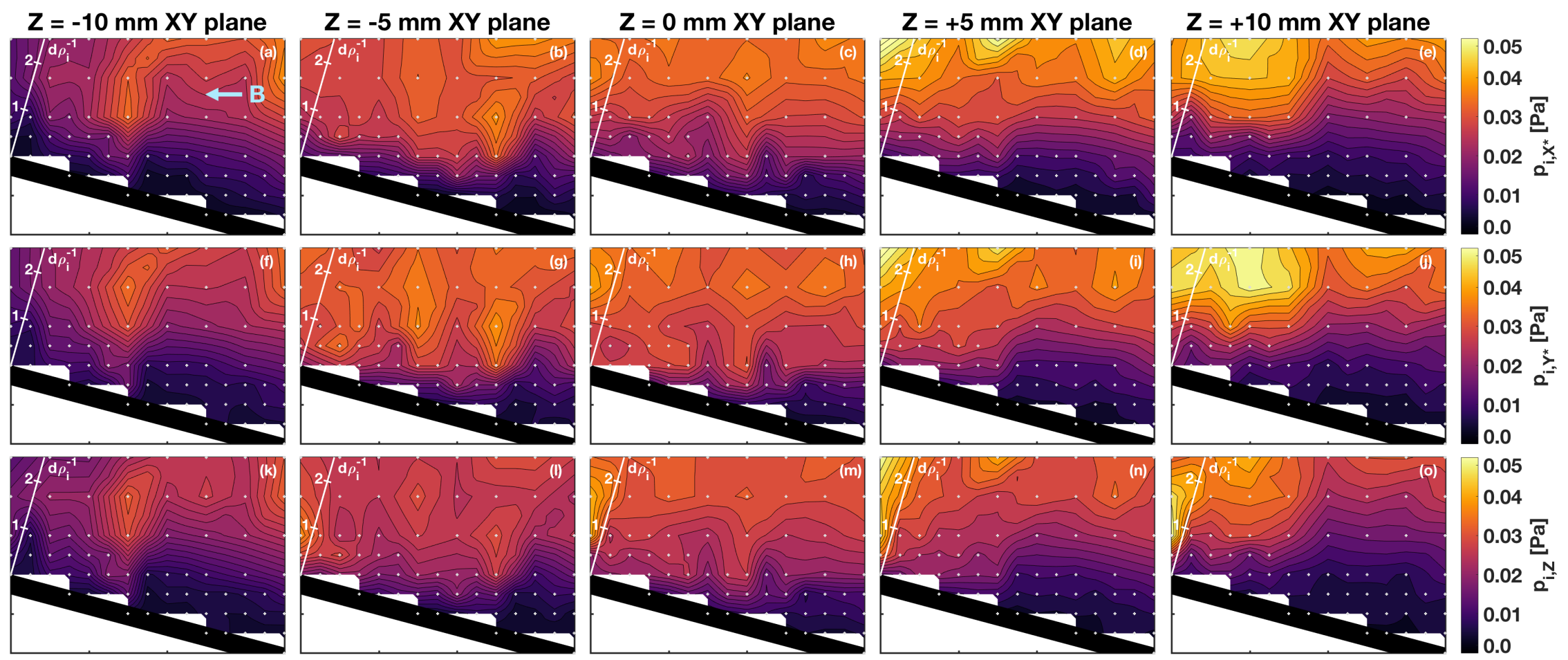

FIGURE 4.11: Spatial variation of $p_{i}(\mathbf{x}, \mathbf{v})$. Bright regions at extreme left in $(\mathrm{k})-(\mathrm{o})$ are an artifact of laser reflections 
by the density variation and the relative uniformity of $T_{i}$. The radial gradient appears in all three directions as it did for the electrons, and it is difficult to identify a gradient with respect to the boundary independent of the radial pressure drop. It is significant that no strong pressure gradients along the axis are observed, with the strongest observed at $z=-10 \mathrm{~mm}$, with point-to-point magnitude $\Delta p_{i, x^{*}} / \Delta y^{*}=0.46 \mathrm{~Pa} / \mathrm{m}$. This corresponds to a pressure force on the ions of order $F_{i}^{p} \sim 10^{-18} \mathrm{~N}$ directed downstream (assuming $\bar{n}_{i}=5 \times 10^{17} \mathrm{~m}^{-3}$ ). The mean of each $z$ plane peaks at $z=0 \mathrm{~mm}$, but the maximum pressure increases with $z$ (Fig. 4.8).

The pressure gradients in Fig. 4.11 suggest a possible explanation for the observation that $\bar{T}_{i, x^{*}}>\bar{T}_{i, z}$ in Sec. 4.5.1: the ions experience an accelerating pressure gradient in $x^{*}$ that they do not experience in $z$. The electric field of the boundary provides an additional accelerating force to the ions along the $x^{*}$ and $y^{*}$ directions. The collisions in the presheath then thermalize the populations, producing higher temperatures along the directions of acceleration. Upstream of the experimental domain, $300 \mathrm{~mm}$ from the boundary, the axial ion temperature is $T_{i, y^{*}}=0.29 \pm 0.02$ $\mathrm{eV}$. By the time the ions enter the experimental domain $T_{i, y^{*}} \geq 0.32 \pm 0.02 \mathrm{eV}$, which suggests additional forces contribute to ion acceleration and heating between the antenna and the boundary region.

\subsubsection{Characteristic velocities in the boundary}

The electron and ion thermal speeds, and the sound speed in the boundary, are calculated from the temperature data using the following relations:

$$
\begin{aligned}
v_{t h e} & =\sqrt{8 k_{\mathrm{B}} T_{e} / \pi m_{e}} \\
v_{t h i} & =\sqrt{k_{\mathrm{B}} T_{i} / m_{i}} \\
c_{s} & =\sqrt{k_{\mathrm{B}}\left(T_{e}+T_{i}\right) / m_{i}} .
\end{aligned}
$$

The range and mean velocity in the boundary are shown in Tab. 4.2. 


\begin{tabular}{llll}
\hline \hline Velocity & Minimum $[\mathrm{m} / \mathrm{s}]$ & Mean $[\mathrm{m} / \mathrm{s}]$ & Maximum $[\mathrm{m} / \mathrm{s}]$ \\
\hline$v_{\text {the }}$ & $1.13 \times 10^{6}$ & $1.32 \times 10^{6}$ & $1.56 \times 10^{6}$ \\
$v_{t h i, x^{*}}$ & 700 & 910 & 1070 \\
$v_{t h i, y^{*}}$ & 790 & 970 & 1160 \\
$v_{t h i, z}$ & 760 & 890 & 1020 \\
$v_{t h i}$ (overall) & 700 & 930 & 1160 \\
$c_{s}$ & 3000 & 3500 & 4090 \\
\hline \hline
\end{tabular}

TABLE 4.2: Characteristic velocities. $v_{t h i, z}$ omits reflection artifacts. $c_{s}$ is tabulated using the minimum, mean, and maximum ion temperatures of all three directions.

\begin{tabular}{llll}
\hline \hline Frequency & Minimum $[\mathrm{rad} / \mathrm{s}]$ & Mean $[\mathrm{rad} / \mathrm{s}]$ & Maximum $[\mathrm{rad} / \mathrm{s}]$ \\
\hline$\omega_{c e}$ & $1.03 \times 10^{10}$ & $1.04 \times 10^{10}$ & $1.05 \times 10^{10}$ \\
$\omega_{p e}$ & $1.35 \times 10^{10}$ & $2.88 \times 10^{10}$ & $4.81 \times 10^{10}$ \\
$\omega_{c i}$ & $1.42 \times 10^{5}$ & $1.43 \times 10^{5}$ & $1.44 \times 10^{5}$ \\
$\omega_{p i}$ & $0.50 \times 10^{8}$ & $1.07 \times 10^{8}$ & $1.78 \times 10^{8}$ \\
$\omega_{\mathrm{RF}}$ & - & $5.97 \times 10^{7}$ & - \\
\hline \hline
\end{tabular}

TABLE 4.3: Plasma and cyclotron frequencies for both charged species. The $\mathrm{RF}$ frequency $\omega_{\mathrm{RF}}$ is included for comparison.

\subsection{Characteristic frequencies in the boundary}

The cyclotron frequency, $\omega_{c}=e B / m$, is calculated from the magnetic field measurements, where $\bar{B}=0.059 \mathrm{~T}$, or $590 \mathrm{G}$. The plasma frequency, $\omega_{p}=\sqrt{n e^{2} / m \epsilon_{o}}$, is calculated from Langmuir probe densities. These quantities are shown in Tab. 4.3 .

The characteristic collision frequencies are calculated using the measured plasma quantities. The argon ions $\left(\mathrm{Ar}^{+}\right)$, gas particles $(\mathrm{Ar})$, and electrons $\left(e^{-}\right)$undergo a 
menagerie of collisions, even if impurity species from the air and chamber materials, such as $\mathrm{O}_{2}, \mathrm{~N}_{2}, \mathrm{C}$ and $\mathrm{Fe}$, are neglected.

Several kinds of ion collisions are considered that fall into three broad categories: ion-neutral elastic scattering, ion-neutral inelastic reactions, and Coulomb collisions with electrons:

$$
\begin{aligned}
& \mathrm{Ar}+\mathrm{Ar}^{+} \longrightarrow \mathrm{Ar}+\mathrm{Ar}^{+} \quad \text { Elastic scattering (SC) } \\
& \mathrm{Ar}+\mathrm{Ar}^{+} \longrightarrow \mathrm{Ar}^{+}+\mathrm{Ar} \quad \text { Charge exchange (CX) } \\
& \mathrm{Ar}+e^{-} \longrightarrow \mathrm{Ar}^{+}+2 e^{-} \quad \text { Ionization (IZ) } \\
& \mathrm{Ar}^{+}+\mathrm{Ar}^{+} \longrightarrow \mathrm{Ar}^{+}+\mathrm{Ar}^{+} \text {Coulomb scattering (II) } \\
& \mathrm{Ar}^{+}+e^{-} \longrightarrow \mathrm{Ar}^{+}+e^{-} \quad \text { Coulomb scattering (IE) } \\
& e^{-}+\mathrm{Ar}^{+} \longrightarrow e^{-}+\mathrm{Ar}^{+} \text {Coulomb scattering (EI) } \\
& e^{-}+e^{-} \longrightarrow e^{-}+e^{-} \quad \text { Coulomb scattering (EE) }
\end{aligned}
$$

In elastic scattering collisions (SC), an argon ion and an argon neutral ballistically scatter, exchanging momentum in the process. Charge exchange (CX) collisions occur when a passing ion acquires an outer shell electron from a neutral atom. This mechanism is important for momentum transfer, as it results in neutral particles impacting the boundary surface after having accelerated to high velocities as ions. In a sense, the neutrals experience the fields in the boundary via this interaction. When it occurs, charge exchange appears in LIF spectra as a fast neutral population and a corresponding depletion of energetic ions. Recombination has a similar effect, in that it results in strong momentum transfer between the ion and neutral populations. Ionization from electron impact is a source mechanism for positively charge ions. Coulomb collisions (II, IE, EI, EE) are interactions between one charged particle's local electric field and the electric charge of another in which the particles' trajectories are redirected.

The frequencies of these collisions depend on the density of the target species and on the ion energy dependent cross-section $\sigma(E)$ of the interaction. The crosssections have been predicted theoretically, however the experimental measurements 


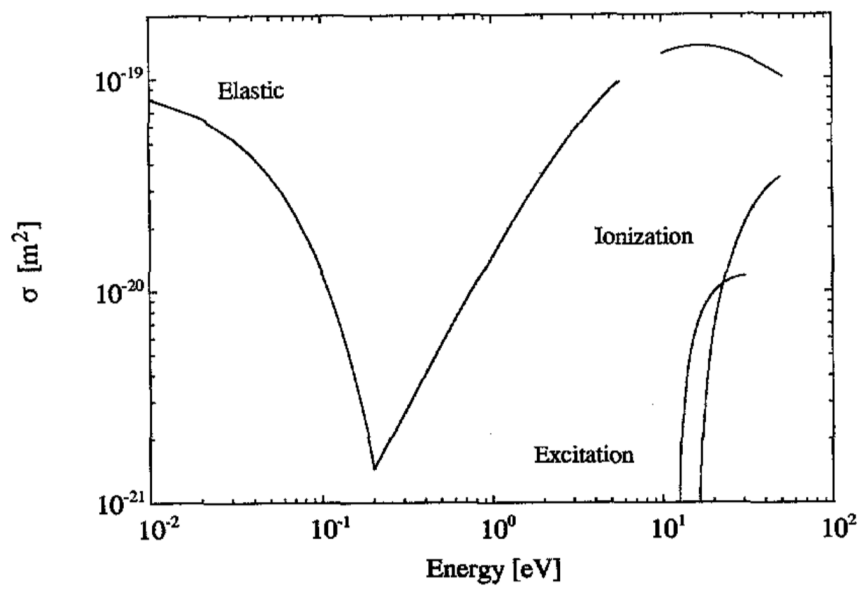

FiguRE 4.12: Experimental ion collision cross-sections [162, 163]. Reprinted from Computer Physics Communications, 87, V. Vahedi and M. Surendra, A Monte Carlo collision model for the particle-in-cell method: applications to argon and oxygen discharges, Pages No. 179-198, Copyright (1995), with permission from Elsevier.

should be used because of the complexity of the problem. Example elastic, ionization, and excitation cross sections are shown in Fig. 4.12 from Refs. [162, 163]. An approximate form for $\sigma_{\mathrm{SC}}$ is given by $[162,164]$

$$
\begin{array}{rlrl}
\sigma_{\mathrm{SC}}(E)\left[\mathrm{m}^{2}\right] & =7.8 \times 10^{-19}-2.0 \times 10^{-19} \sqrt{E} \quad 0 \leq E \leq 4 \\
& =2 \times 10^{-19}+5.5 \times 10^{-19} E^{-1 / 2}, & E \geq 4
\end{array}
$$

however $\bar{\sigma}_{\mathrm{SC}}$ and $\bar{\sigma}_{\mathrm{CX}}$ are determined from the experimental data in Ref. [1], which are reproduced in Fig. 4.13. A numerical fit to the CX data gives the relation

$$
\sigma_{\mathrm{CX}}(E)\left[\mathrm{m}^{2}\right]=10^{20}\left[59.28-3.257 \times 10^{-4} E-4.641 \ln (0.35)\right] .
$$

Estimates of collision frequencies from scattering and charge exchange interactions are calculated by the relation

$$
\nu=n_{n} \sigma \bar{v}_{t h}
$$

and require estimates of the neutral density $n_{n}$ and the mean velocity $\bar{v}_{t h}$ of the moving particles, for which the mean thermal speeds in Tab. 4.2 are used. 

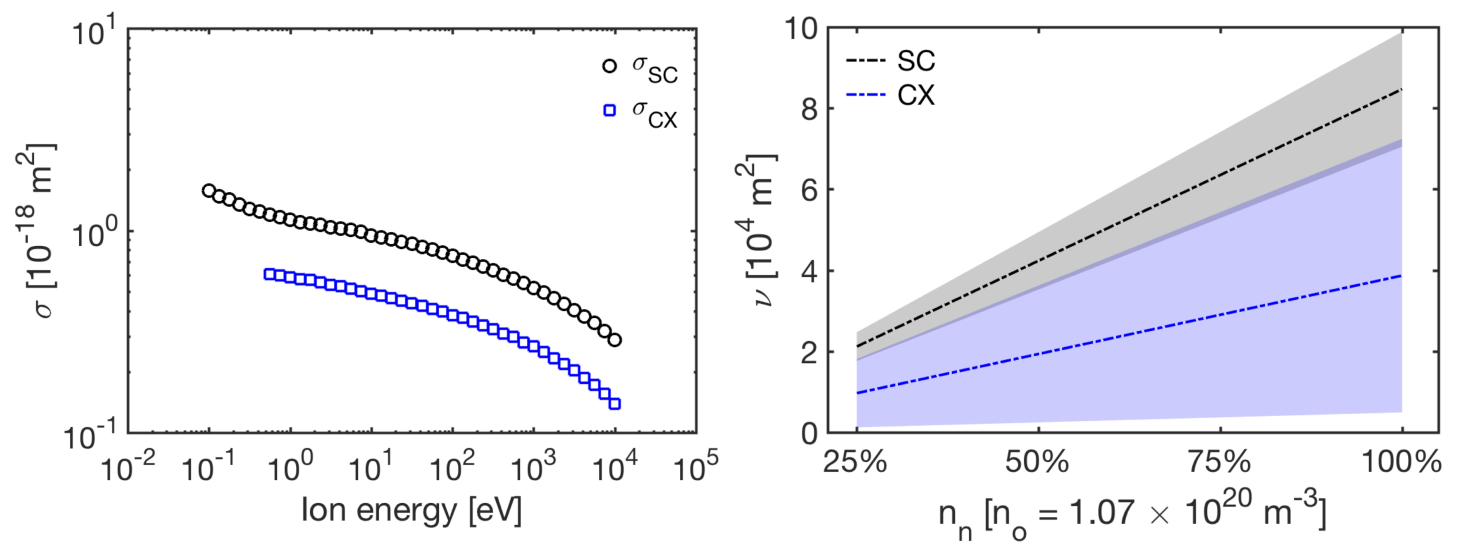

FiguRE 4.13: Experimental $\sigma_{\mathrm{CX}}$ and $\sigma_{\mathrm{SC}}$, with $\nu_{\mathrm{CX}}$ and $\nu_{\mathrm{SC}}$ calculated for a range of neutral densities. Pale bars represent standard deviation in collision frequency from standard deviation in cross section. Lines represent the collision frequency calculated with the mean cross section.
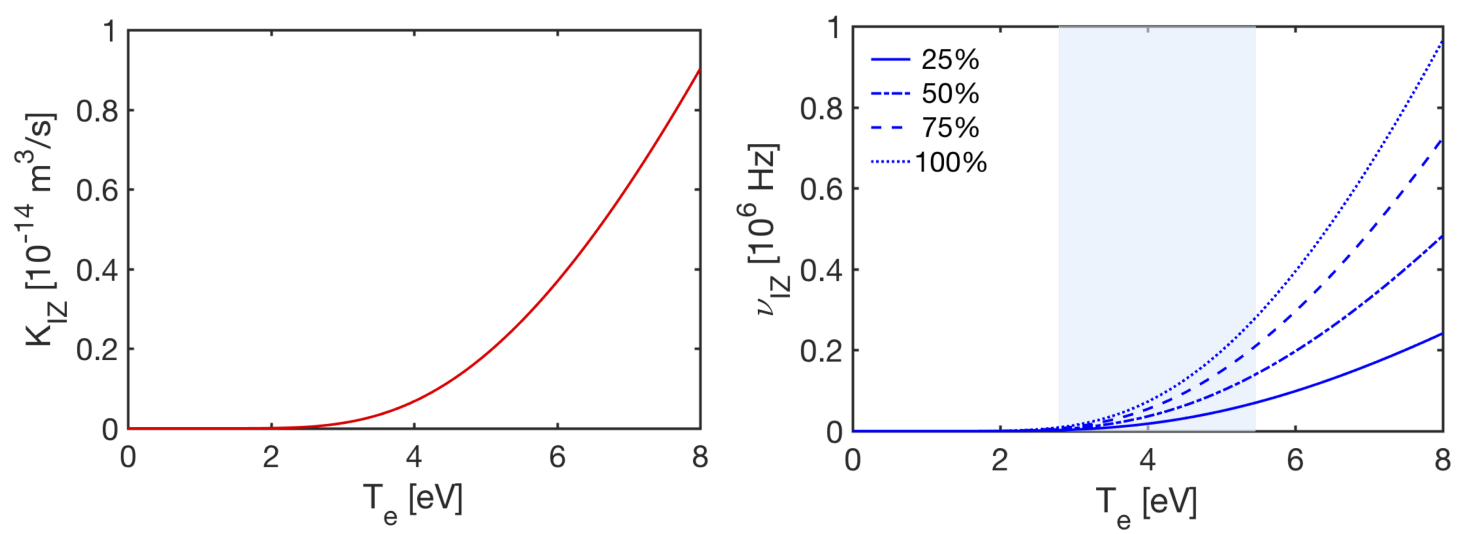

Figure 4.14: Dependence of $K_{\mathrm{IZ}}$ and $\nu_{\mathrm{IZ}}$ on $n_{n}$ and $T_{e}$. The pale blue shaded region marks range of measured electron temperatures. Lines represent percentage of $n_{n}=1.07 \times 10^{20} \mathrm{~m}^{-3}$.

The ionization rate $K_{\mathrm{Iz}}$ is approximated by the relation [121]

$$
K_{\mathrm{IZ}}\left(T_{e}\right)\left[\mathrm{m}^{3} / \mathrm{s}\right]=2.34 \times 10^{-14} T_{e}^{0.59} \exp \left(-17.44 / T_{e}\right)
$$

and shown over a range of $T_{e}$ in Fig. 4.14. The ionization frequency is calculated by $\nu_{\mathrm{IZ}}=n_{n} K_{\mathrm{IZ}}$. (A full treatment of collisions requires integrating the collision integral. Approximate solutions will be used here to determine scaling instead of using the more involved analysis reserved for simulations.)

Fig. 4.14 demonstrates a major challenge of simulating low temperature plasma 


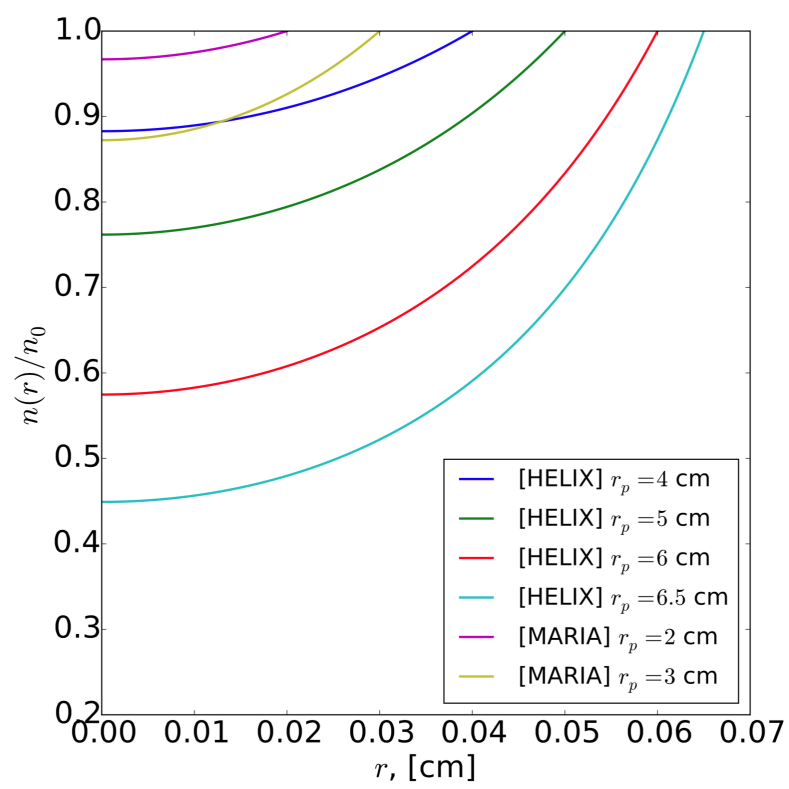

Figure 4.15: Neutral depletion on-axis in HELIX for several plasma diameters [164]. Reproduced with permission from R. Khaziev, Multiscale Numerical simulations of the magnetized plasma sheath with massively parallel electrostatic particle-in-cell code (C) 2018 R. Khaziev.

boundaries: the collisionality is very sensitive to the neutral density, which cannot be measured absolutely in pure argon and is difficult to estimate. A naive estimate of the neutral density of the gas interacting with the plasma is obtained by the ideal gas law, $n_{n}=p_{n} / k_{\mathrm{B}} T_{n}$, where $p_{n}=3.6 \mathrm{mTorr}(0.48 \mathrm{~Pa})$ is measured by the pressure gauges in the chamber wall and the neutral temperature $T_{n} \approx 0.028 \mathrm{eV}$ is measured by LIF. These conditions suggest $n_{n}=1.07 \times 10^{20} \mathrm{~m}^{-3}$. This method assumes that the neutral profile is flat across the chamber and that there is no change in pressure between the location of the pressure gauge and the experiment. Both assumptions are faulty. Measurements in HELIX have demonstrated high ionization rates in the core of the helicon plasma, with corresponding depletion of the neutral population [80]. In the CHEWIE device, experiments in krypton show neutral density and profile shape dependence on magnetic field magnitude, fill pressure, RF frequency, and RF power [84].

The radial neutral profile in HELIX was recently (Ref. [164]) estimated for several plasma diameters using a method proposed by Gilland et al. [79]. The results are shown in Fig. 4.15. According to these calculations, the neutral density along the axis is between $44 \%$ and $90 \%$ of the pressure measured at the edge. Profiles from 
Keesee et al. [80], discussed in Sec. 1.3.4, suggest a comparable range, with some authors claiming as much as a factor of 4 neutral depletion from maximum neutral density [82]. This drastically changes the frequencies of collisions involving neutral particles, which are at least linearly dependent on $n_{n}$.

The cross sections, calculated from the data in Fig. 4.13, are $\bar{\sigma}_{\mathrm{CX}}=6.415 \times 10^{-19}$ $\mathrm{m}^{2}$ and $\bar{\sigma}_{\mathrm{SC}}=8.5078 \times 10^{-19} \mathrm{~m}^{2}$. Mean collision frequencies at $75 \%$ neutral fraction are $\nu_{\mathrm{SC}}=6.34 \times 10^{4} \mathrm{~s}^{-1}$ and $\nu_{\mathrm{CX}}=4.79 \times 10^{4} \mathrm{~s}^{-1}$. (Ref. [164] calculates the full collision integral, using $100 \%$ neutral density and a flat profile. Collision frequencies calculated in this way are typically larger by a factor of about 2.)

Coulomb collisions depend on the Coulomb logarithm, $\lambda \doteq \ln \Lambda$, which describes the ratio of the maximum and minimum applicable values of the impact parameter during a collision. It is different depending on the two particles interacting. Detailed numerical approximations of the Coulomb logarithm from experimental data are provided by the NRL Plasma Formulary [123], however in most cases $\lambda \approx 10$. When $T_{i} m_{e} / m_{i}<T_{e}<10 \mathrm{eV}$, for electron mass $m_{e}=9.11 \times 10^{-31} \mathrm{~kg}$, argon ion mass $m_{i}=6.6335 \times 10^{-26} \mathrm{~kg}$, and singly-ionized atomic argon:

$$
\begin{aligned}
\lambda_{\mathrm{EE}} & =23.5-\ln \left[\left(n_{e} / 10^{6}\right)^{1 / 2} T_{e}^{-5 / 4}\right]-\left[10^{-5}+\left(\ln T_{e}-2\right)^{2} / 16\right]^{1 / 2} \\
\lambda_{\mathrm{EI}} & =\lambda_{\mathrm{IE}}=23-\ln \left[\left(n_{e} / 10^{6}\right)^{1 / 2} T_{e}^{-3 / 2}\right] \\
\lambda_{\mathrm{II}} & =23-\ln \left[\left(2 n_{i}\right)^{1 / 2} T_{i}^{-3 / 2}\right]
\end{aligned}
$$

These quantities are shown as functions of $T_{e}, T_{i}$ and $n$ in Fig. 4.16. 


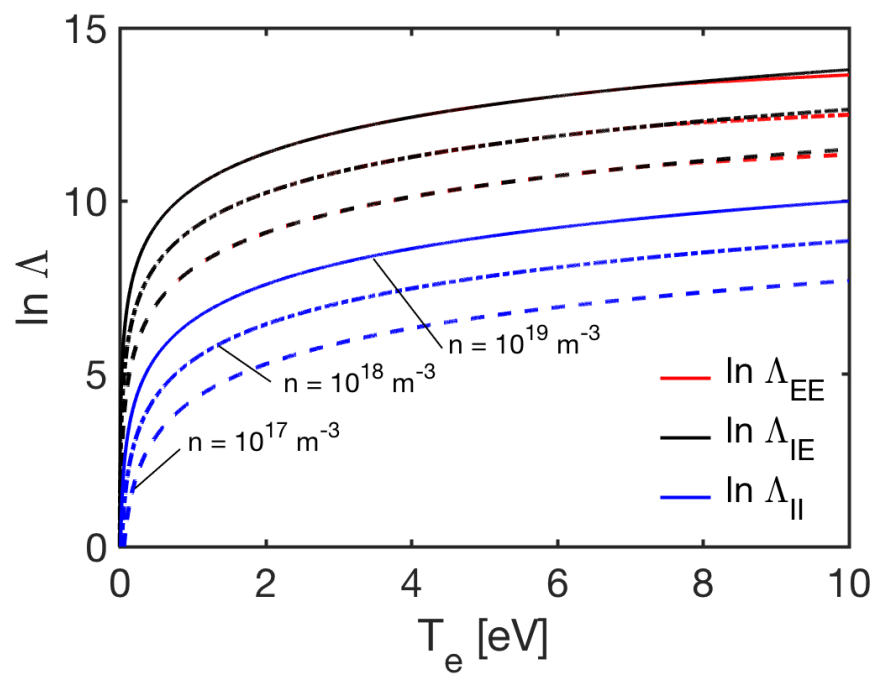

Figure 4.16: Dependence of $\ln \Lambda$ on $T_{e}, T_{i}$ and $n$.

The electron-electron, electron-ion, ion-electron, and ion-ion collision frequencies are given by the Lenard-Balescu kernel:

$$
\begin{aligned}
& \nu_{\mathrm{EE}}\left[\mathrm{s}^{-1}\right] \approx \frac{n_{e} e^{4}}{4 \pi \epsilon_{o}^{2} m_{e}^{1 / 2} T_{e}^{3 / 2}} \lambda_{\mathrm{EE}}\left(n_{e}, T_{e}\right) \\
& \nu_{\mathrm{EI}}\left[\mathrm{s}^{-1}\right] \approx \frac{\sqrt{2}}{12 \pi^{3 / 2}} \frac{n_{i} e^{4}}{\epsilon_{o}^{2} m_{e}^{1 / 2}\left(k_{\mathrm{B}} T_{e}\right)^{3 / 2}} \lambda_{\mathrm{EI}}\left(n_{e}, T_{e}\right) \\
& \nu_{\mathrm{IE}}\left[\mathrm{s}^{-1}\right] \approx\left(m_{e} / m_{i}\right) \nu_{\mathrm{EI}} \\
& \nu_{\mathrm{II}}\left[\mathrm{s}^{-1}\right] \approx \frac{n_{i} e^{4}}{12 \pi^{3 / 2} \epsilon_{o}^{2} m_{i}^{1 / 2}\left(k_{\mathrm{B}} T_{i}\right)^{3 / 2}} \lambda_{\mathrm{II}}\left(n_{i}, T_{i}\right)
\end{aligned}
$$

from [123, p. 34], [165], and [166, p. 217], where permittivity of free-space $\epsilon_{o}=$ $8.854 \times 10^{-12} \mathrm{~F} / \mathrm{m}$. The $T_{i}$ and $T_{e}$ dependence of these collision frequencies is shown in Fig. 4.17.

Recent experiments by Yip et al. [167] and Claire et al. [24] have challenged the assumption that ion-ion collisions are necessarily negligible in the presheath. In response, Baalrud et al. have extended the traditional Lenard-Balescu theory in Eq. (4.2) to include ion-acoustic and ion-ion two-stream instability enhanced collisions [165]. They argue that these instabilities effectively thermalize non-Maxwellian 

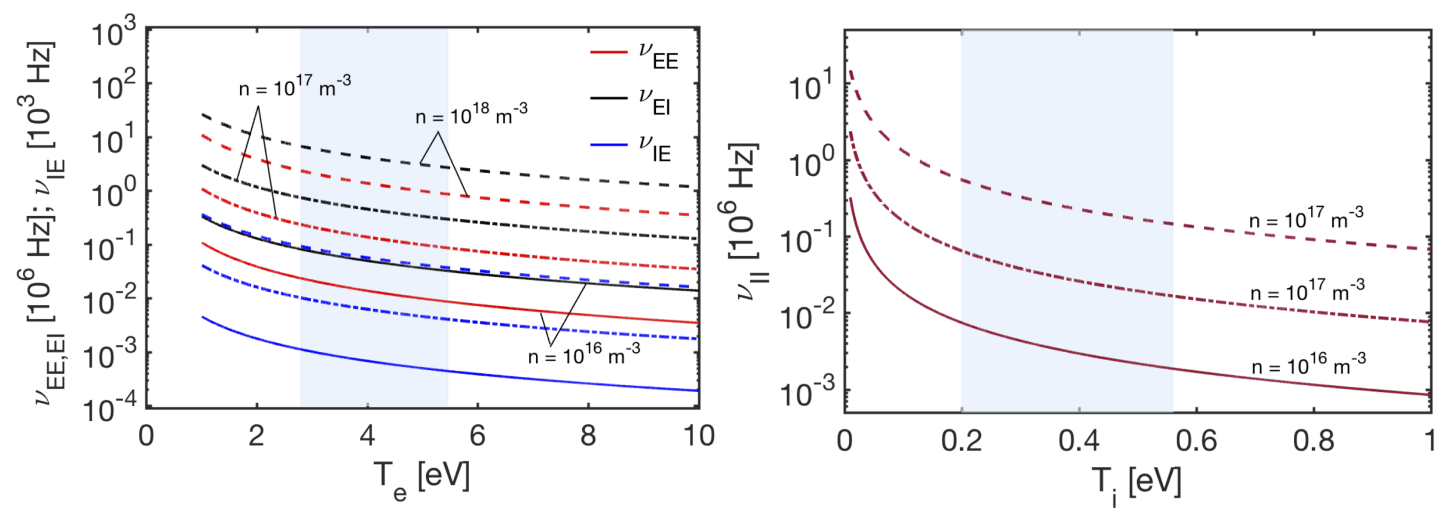

Figure 4.17: Dependence of Coulomb collision frequencies on $T_{e}, T_{i}$ and $n_{e} \approx n_{i}$. Pale blue regions mark the extent of $T_{e}$ and $T_{i}$ observed.

tails arising in IVDFs from ion-neutral collisions. The authors propose a parameter (for unmagnetized plasmas)

$$
\eta \doteq \frac{\lambda_{\mathrm{ps}}}{\lambda_{D e}} \sqrt{\frac{\pi m_{e}}{16 m_{i}}}
$$

which determines the size of the electron-electron collisional enhancement, as the enhanced collision frequency $\nu_{\mathrm{EE}}^{*} \propto \exp \left(\eta x / \lambda_{\mathrm{ps}}\right) . \lambda_{D e}$ is the electron Debye length

$$
\lambda_{D e}=\sqrt{\frac{\epsilon_{o} k_{\mathrm{B}} / e^{2}}{n_{e} / T_{e}+n_{i} / T_{i}}},
$$

and $\lambda_{\mathrm{ps}}$ is the presheath scale length, which is estimated by the ion-neutral collision length $\lambda_{\mathrm{IN}}=\bar{v}_{t h i} / \nu_{\mathrm{IN}}$. For all but the most extreme plasma parameters observed, $\eta \lesssim 0.83$, and on average $\eta \approx 0.12$, indicating that instabilities do not contribute to IVDF thermalization in a significant way. In the MARIA experiment [22], at lower $\psi$, the presheath scale was 3-4 times larger, and instability enhanced collisions may have occurred in those boundaries.

The collision frequencies are summarized in Tab. 4.4, with total ion-neutral collision frequency $\nu_{\mathrm{IN}}=\nu_{\mathrm{CX}}+\nu_{\mathrm{SC}}$, total ion collision frequency $\nu_{\mathrm{I}}=\nu_{\mathrm{IN}}+\nu_{\mathrm{II}}+\nu_{\mathrm{IE}}$, and total electron collision frequency $\nu_{\mathrm{E}}=\nu_{\mathrm{IZ}}+\nu_{\mathrm{EI}}+\nu_{\mathrm{EE}}$. Ion-neutral collisions are expected to be comparable to ion-ion collisions and ionization. 


\begin{tabular}{llll}
\hline \hline Frequency & Minimum $\left[s^{-1}\right]$ & Mean $\left[s^{-1}\right]$ & Maximum $\left[s^{-1}\right]$ \\
\hline$\nu_{\mathrm{SC}}$ & $2.10 \times 10^{4}$ & $6.34 \times 10^{4}$ & $8.47 \times 10^{4}$ \\
$\nu_{\mathrm{CX}}$ & $1.20 \times 10^{4}$ & $4.79 \times 10^{4}$ & $7.96 \times 10^{4}$ \\
$\nu_{\mathrm{IZ}}$ & $2.50 \times 10^{3}$ & $4.92 \times 10^{4}$ & $2.80 \times 10^{5}$ \\
$\nu_{\mathrm{II}}$ & $1.19 \times 10^{5}$ & $3.3 \times 10^{5}$ & $7.4 \times 10^{5}$ \\
$\nu_{\mathrm{IE}}$ & 2.4 & 17 & 66 \\
$\nu_{\mathrm{EI}}$ & $1.70 \times 10^{5}$ & $1.3 \times 10^{6}$ & $4.8 \times 10^{6}$ \\
$\nu_{\mathrm{EE}}$ & $4.90 \times 10^{4}$ & $4.0 \times 10^{5}$ & $1.7 \times 10^{6}$ \\
$\nu_{\mathrm{IN}}$ & $3.3 \times 10^{4}$ & $1.11 \times 10^{5}$ & $1.64 \times 10^{5}$ \\
$\nu_{\mathrm{I}}$ & $1.52 \times 10^{5}$ & $4.41 \times 10^{5}$ & $9.14 \times 10^{5}$ \\
$\nu_{\mathrm{E}}$ & $2.22 \times 10^{5}$ & $1.75 \times 10^{6}$ & $6.78 \times 10^{6}$ \\
\hline \hline
\end{tabular}

TABLE 4.4: Collision frequencies for measured range of $T_{e}, T_{i}$, and $n$. Min., mean, and max. $n: 5.7 \times 10^{16} \mathrm{~m}^{-3}, 2.8 \times 10^{17} \mathrm{~m}^{-3}, 7.3 \times 10^{17} \mathrm{~m}^{-3}$. Min., mean, and max. $T_{e}: 2.84 \mathrm{eV}, 3.92 \mathrm{eV}, 5.46 \mathrm{eV}$. Min., mean, and max. $T_{i}: 0.20$ $\mathrm{eV}, 0.36 \mathrm{eV}, 0.56 \mathrm{eV} \cdot \nu_{\mathrm{SC}}-$ ion-neutral scattering collision frequency; $\nu_{\mathrm{CX}}-$ ion-neutral charge exchange collision frequency; $\nu_{\mathrm{IZ}}$ - ionization; $\nu_{\mathrm{II}}-$ ion-ion Coulomb collision frequency; $\nu_{\mathrm{IE}}$ and $\nu_{\mathrm{EI}}$ - electron-ion Coulomb collision frequency; $\nu_{\mathrm{EE}}$ - electron-electron Coulomb collision frequency; $\nu_{\mathrm{IN}}$ - combined ion-neutral collision frequency; $\nu_{\mathrm{I}}-$ total ion collision frequency; $\nu_{\mathrm{E}}-$ total electron collision frequency. For collisions with neutrals, the minimum is calculated using $20 \%$ and the mean is calculated using $75 \%$. Assuming a flat profile and $0 \%$ ionization, $n_{n}=1.07 \times 10^{20} \mathrm{~m}^{-3}$.

The influence of the magnetic field depends on the collisions the particles make while traveling along field lines. If the particles experience no collisions, they follow field lines closely with little transport across them. These particles are referred to as "magnetized." At the other extreme, particles that undergo many collisions per gyration do not travel along any one field line for very long before transferring to the next, and the magnetic field does not tightly define the structure of the motion of these particles. These particles are referred to as "unmagnetized" or "collisional." The quantity that determines the collisionality regime of each species is called the Hall parameter, the ratio of gyro-frequency to collision frequency $\omega_{c} \tau$ 
where $\tau=\nu^{-1}$. (It is convention to compare frequencies in this way, even if the resulting units are radians.) When $\omega_{c} \tau \gg 1$, the particles follow the field lines closely and the background field strongly determines the structure of the plasma and its flows. When $\omega_{c} \tau \ll 1$, collisions disrupt this organization and particles easily diffuse across field lines.

The ions have a Hall parameter of $\omega_{c i} \tau_{i}=0.5 \pm 0.3$, indicating an intermediate regime. The magnetic field plays an important, but not dominant, role in determining the behavior of the ions. The range given for $\omega_{c i} \tau_{i}$ arises from reasonable assumptions about the range $n_{n}$ and $\bar{\sigma}$ may take. (Author note: Although this number arises from discussions with Dr. Rinat Khaziev of Ref. [164], fault for any error in it remains with me.) The Hall parameter for the electrons indicates clear magnetization: $\omega_{c e} \tau_{e} \geq 1500$.

\subsection{The metastable Ar-II drift velocities measured by LIF show a 3D flow field}

The primary aim of LIF measurements in the plasma boundary is to observe ion flows and the potential interaction of ion and neutral species. Ion flow fields are constructed from the $1^{\text {st }}$ velocity moment of the distribution function, which for Maxwellian distributions occurs at the peak of the distribution. The velocities are measured in the laboratory system $\left(x^{*}, y^{*}, z\right)$ before being rotated into the $(x$, $y, z)$ simulation coordinate system (see Sec. 2.3.2). The $\left(u_{x}, u_{y}, u_{z}\right)$ velocities, normalized to the local sound speed $c_{s}(\mathbf{x})$ are shown in Fig. 4.18. This data set contains the only known 3D3V measurement of ion flows in a boundary region, the only known measurements in the $u_{z}$ direction, and the only known ion flow measurements of the boundary region at $\psi \geq 60^{\circ}$.

$300 \mathrm{~mm}$ upstream of the boundary, at the chamber mechanical axis, the drift velocity measured parallel to the magnetic field is $u_{\|}=110 \pm 35 \mathrm{~m} / \mathrm{s}$. Normalized to the mean sound speed in the boundary $\left(\bar{c}_{s}=3500 \mathrm{~m} / \mathrm{s}\right)$, this corresponds to 

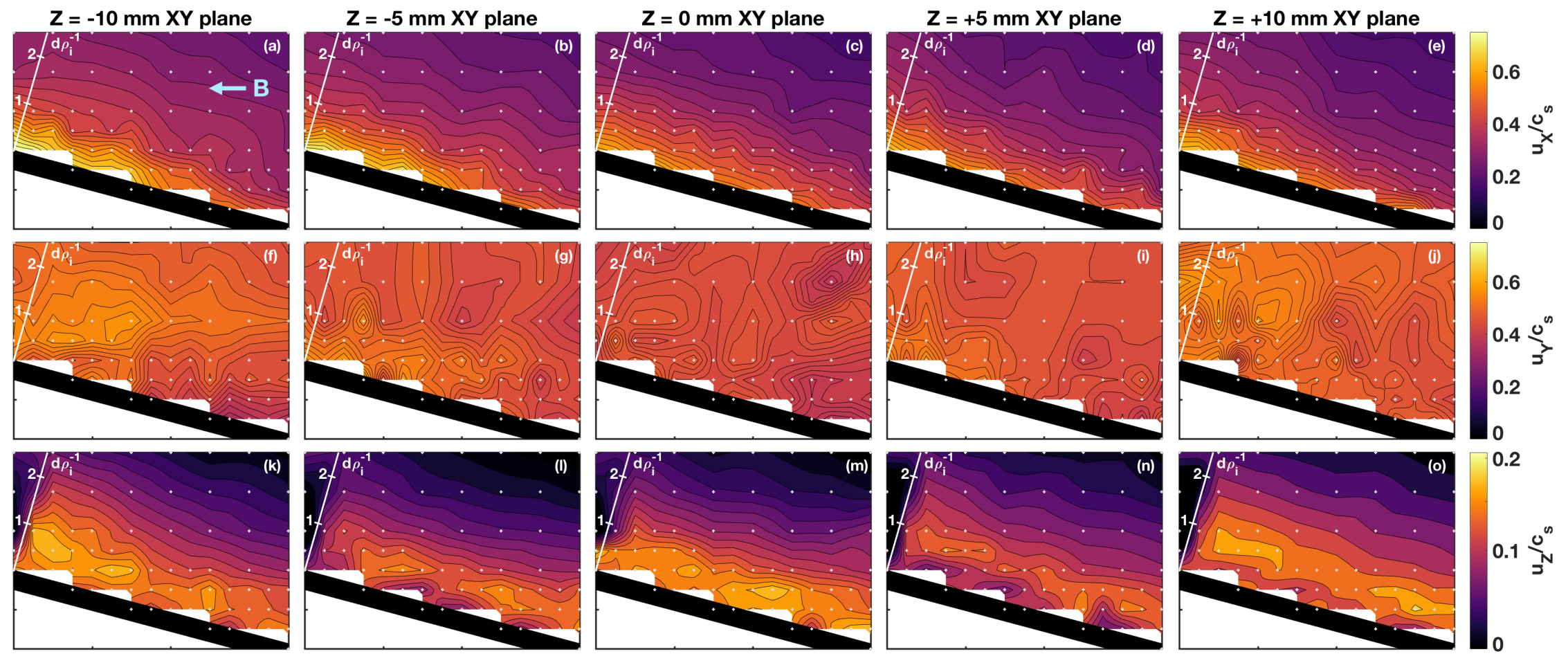

FIGURE 4.18: 3D map of Ar-II metastable drift velocities from LIF data. Flows are presented in the simulation frame $\left(u_{x}, u_{y}, u_{z}\right)$ and normalized to the local sound speed. Dark regions at the downstream end of graphs (k)-(o) are artifacts from laser reflections and do not represent actual velocities. 
parallel Mach number $u_{\|} / c_{s} \approx 0.03$. From this initial velocity, the ions accelerate to $u_{\|} / c_{s} \geq 0.41$ at the upstream entrance to the boundary domain. Upon entering the domain, $u_{y} / c_{s}$ - which is at a small angle to $u_{y^{*}} / c_{s}=u_{\|} / c_{s}$ - does not significantly accelerate.

The ion drift velocities increase substantially in the direction normal to the boundary. With the addition of this drift, the ion motion is at least two-dimensional. From $u_{x} / c_{s} \geq 0.16$ at the boundary entrance, the ions accelerate along $-\partial \phi_{p} / \partial x$ up to $u_{x} / c_{s}=0.78$ just above the surface. The Bohm criterion, wherein $u_{x} \geq c_{s}$ at the sheath edge, is never observed. This is at least in part due to a large LIF sample region with respect to the size of the sheath. In Sec. 3.2.3.3, the Debye length was calculated to be $0.03-0.05 \mathrm{~mm}$, and a conservative estimate for the sheath width is $\max \left(h_{e}, h_{i}\right) \approx 0.6 \mathrm{~mm}$. The effective collection volume for the transformed velocities is at least $1.2 \mathrm{~mm}$. Any measurement of the sheath therefore necessarily measures a substantial ion population outside the Debye sheath, and the weighted average is observed in the LIF spectrum. This deflection from field-parallel to drift toward the boundary was observed by Siddiqui et al. [22] at $\psi \leq 60^{\circ}$. These data confirm that the deflection phenomenon occurs to $\psi \leq 74^{\circ}$.

These two drift components exist in the $\mathbf{E}-\mathbf{B}$ plane. As shown in (k)-(o) of Fig. 4.18 , the ion drift acquires a third, non-linear component and diverts from $u_{\|}$not only along $x$ but in the direction parallel to $\mathbf{E} \times \mathbf{B}$ as well. The presence of these drifts was predicted in fluid theory boundary models by Reimann [168], Ahedo [11] and others and was required by Siddiqui et al. to match fluid models to the 2D data in their experiments [22]. The ion drifts observed along $z$ constitute direct, non-perturbative measurements of ion transport across field lines in a boundary with an oblique magnetic field. In addition, for $u_{z}(z \leq 0)$, the ions flow against the pressure gradient $\partial p_{i} / \partial z$ seen in Figs. 4.11 and 4.8 .

Drift in the $\mathbf{E} \times \mathbf{B}$ direction is not due solely to the rotation of the plasma column. The acceleration of the ions clearly depends on their proximity to the boundary surface and not on plasma radius. At the top of the experimental domain, where 
$d / \rho_{i}=3.8, u_{z} / c_{s}<0.01 . u_{z} / c_{s}$ increases linearly to a maximum of $u_{z} / c_{s}=$ $0.18-0.20$ between $d / \rho_{i}=0.5-0.7$.

Within $d / \rho_{i} \lesssim 0.5, u_{z} / c_{s}$ decreases to 0.1 . The cause of this decrease is not known. It is unlikely to be a reflection effect from laser bombardment of the mounting flange, which is $50 \mathrm{~cm}$ away, since the return beam would be diffuse. In addition, the flange is covered by a large area razor blade beam dump that strongly attenuates the reflected beam. It is unlikely caused by a large sample volume, since the drift velocities begin to decrease more than one laser diameter away from the surface. It is also not a consequence of the interpolation algorithm; the original data is shown in Fig. 4.21. The phenomenon occurs at the velocity distribution level. Fig. 4.22 shows the $v_{z}$ velocity distributions, normalized to the maximum signal of the set, recorded at different distances to the boundary surface. These measurements reveal shifted Maxwellian distributions that slow within a few tenths of a gyroradius from the surface. The effects of inelastic collisions like charge exchange and ionization do not appear in the Maxwellian $v_{z}$ distributions like the ones shown in Fig. 4.22, however they are expected to be small and are likely unresolvable. The upper bound on any divergence from a Maxwellian-distributed population is $2 \%$.

\subsection{Testing Chodura's model}

As discussed in Sec. 1.1.1, the Bohm-Chodura criterion predicts that $u_{x} / c_{s} \geq \cos \psi$ occurs a distance $\lambda_{\text {mps }}=\sqrt{6}\left(c_{s} / \omega_{c i}\right) \sin \psi$ from the Debye sheath edge. Siddiqui et al. conducted a series of experiments in the MARIA device in the vicinity of a boundary arranged at several angles to the magnetic field. LIF measurements were taken of the ion flow to the boundary in two dimensions. Fig. 4.19 shows a comparison between these measurements and Chodura's prediction for the length of the magnetic presheath [23]. The data from the MARIA experiments clearly show that for every experiment with angle $\psi \leq 60^{\circ}$ the Bohm-Chodura criterion is met closer to the boundary than Chodura proposed. In other words, the ions are drifting more slowly than predicted. The predicted and observed locations of 
(a) $\psi=16^{\circ}$

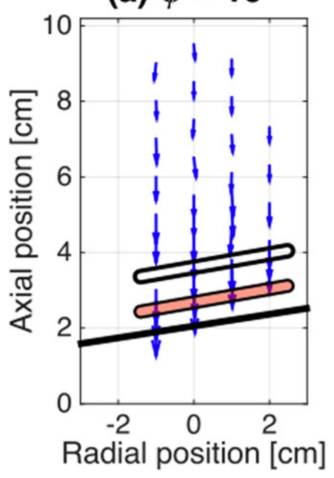

(b) $\psi=30^{\circ}$

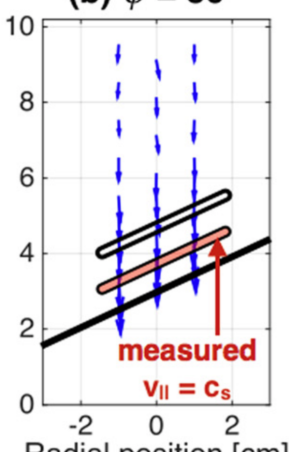

(c) $\psi=45^{\circ}$

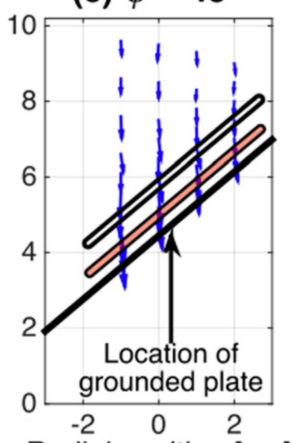

Radial position [cm]
Chodura

prediction for

$\mathrm{v}_{\|}=\mathbf{c}_{\mathrm{s}}$

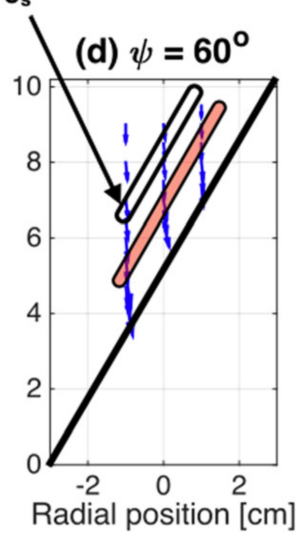

Figure 4.19: Comparison between $\mathbf{u}_{i}$ measurements in MARIA and Chodura's prediction [23]. Blue arrows show 2D ion velocities. White oval shows the location of Chodura's prediction for the location where $u_{\|}=c_{s}$. Red ovals show the location measured by Siddiqui et al. Reprinted from M. U. Siddiqui, D. S. Thompson, C. D. Jackson, J. F. Kim, and N. Hershkowitz, Physics of Plasmas, 23, 057101 (2016), with the permission of AIP Publishing.
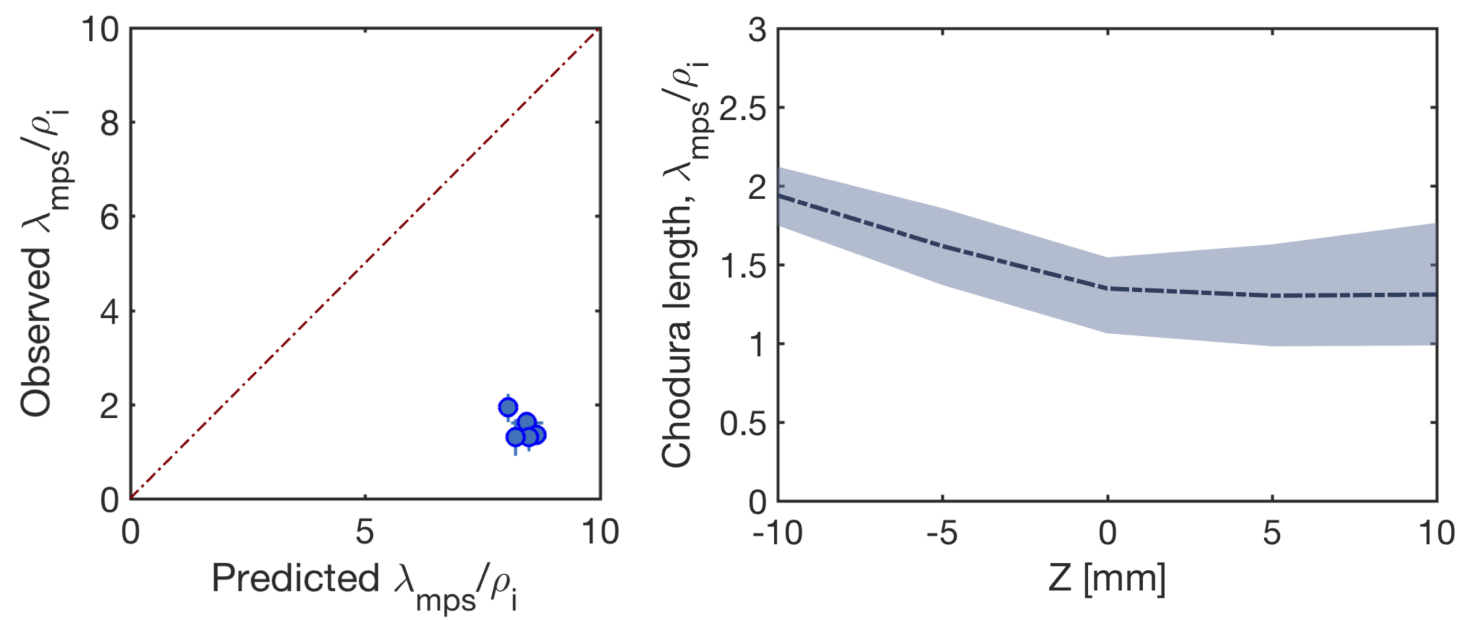

Figure 4.20: (Left) Comparison of predicted and observed $\lambda_{\text {mps }}$. (Right) Profile of observed $\lambda_{\text {mps }} / \rho_{i}$. The dashed line indicates the mean, and the shaded region indicates the range. 
$Z=-10 \mathrm{~mm} X Y$ plane

$Z=-5 \mathrm{~mm} X Y$ plane

$Z=0 \mathrm{~mm} X Y$ plane

$\mathrm{Z}=+\mathbf{5} \mathrm{mm} \mathrm{XY}$ plane

$Z=+10 \mathrm{~mm} \mathrm{XY}$ plane

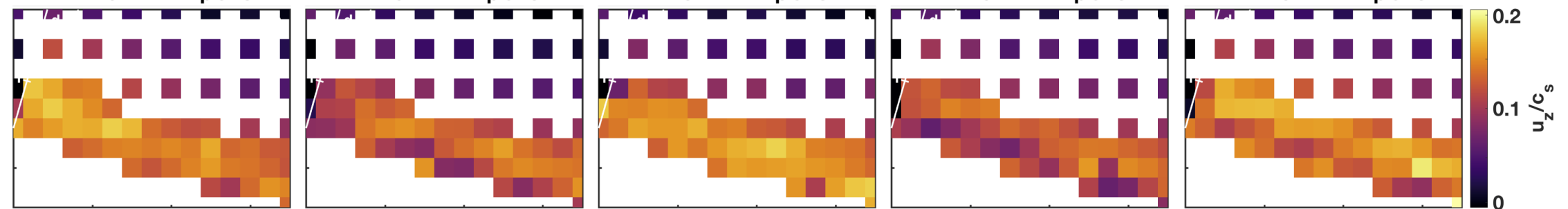

FigURE 4.21: Data showing decrease in $u_{z}$ near boundary before interpolation. 


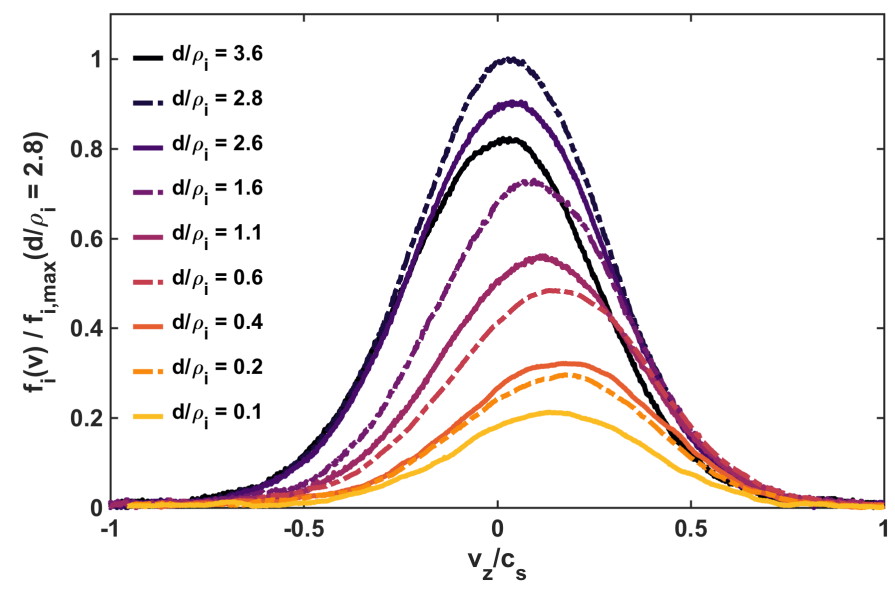

FiguRE 4.22: $v_{z}$ distribution function cascade with distance to the boundary surface.

the Bohm-Chodura criterion in the HELIX measurements are shown in Fig. 4.20. The Chodura model is intended for plasma boundaries in which $\lambda_{i} / \rho_{i} \gg 1$, which is not the case in the HELIX experiments, and therefore is expected to be a poor description of the boundary structure. That said, these measurements corroborate the observations of Siddiqui et al. [22], which $\operatorname{span} \lambda_{i} / \rho_{i}=0.5-4$ in finding that the predicted distance of the magnetic presheath edge is typically farther from the wall than observed, by a factor of about 4 in HELIX, and extend them to $\psi=74^{\circ}$.

Combined 3D LIF and probe data allow the Chodura model to be investigated at multiple locations in $z$ along the boundary. Figs. 4.23 and 4.24 show the location of the Chodura boundary in 3D. The criterion is satisfied at different locations for different locations in $z$. The $z$ dependence of $\lambda_{\text {mps }}$ is shown at right in Fig. 4.20. Observed $\lambda_{\text {mps }}$ ranges from $6.1 \mathrm{~mm}\left(\approx \rho_{i}\right)$ to $13.2 \mathrm{~mm}\left(2.1 \rho_{i}\right)$, with an average distance of $9.3 \mathrm{~mm}\left(1.5 \rho_{i}\right)$. Siddiqui et al. found that at $\psi=60^{\circ}$, the Chodura criterion is predicted to be met at $\lambda_{\mathrm{mps}} / \rho_{i}=6.3$, but is observed to occur at $\lambda_{\text {mps }} / \rho_{i}=0.6 \pm 0.01$ [22]. It is worth noting that Chodura, in his initial 1D1V model, excluded flows $u_{y}$ and $u_{z}$, which simulations suggest couple with $u_{z}$ (see, for example, Ref. [11]). The inclusion of these drifts alone is sufficient to alter the location of the Chodura layer boundary. 
Siddiqui et al. proposed a different definition of the magnetic presheath, determined at the point where the total ion flow diverges by $0.01 c_{s}$ from flow purely parallel to the magnetic field. They compare the $0.01 c_{s}$ distance to the half-maximum distance $d_{h m}$ and find that at $\psi \lesssim 45^{\circ}, \lambda_{\mathrm{mps}} / d_{h m} \approx 1$, however at $\psi=60^{\circ}$ the $\lambda_{\mathrm{mps}} / d_{h m} \approx 3$. Fig. 4.25 shows the total ion flow difference from parallel flow $u_{\text {tot }}-u_{\|}$with respect to distance from the surface for the HELIX measurements at $\psi=74^{\circ}$ and $\omega_{c i} \tau_{i}=0.5$. The total flow and the flow in the $2 \mathrm{D}$ plane of the magnetic field $u_{x y}$ are considered. $\lambda_{\mathrm{mps}} / d_{h m} \approx 3.01 \pm 0.04$ when $u_{z}$ is included, and $\lambda_{\mathrm{mps}} / d_{h m} \approx 3.83 \pm 0.04$ when comparing $u_{\|}$to $u_{x y}$ alone, continuing the trend toward higher $\lambda_{\text {mps }} / d_{h m}$ at larger $\psi$ when $u_{z}$ is excluded. The trend of $\psi$ when total velocity is considered remains to be determined, since $u_{z}$ is unknown at other magnetic angles. The potential gradient is expected to increase with increasing $\psi$, in order to allow the ions to be diverted away from $u_{\|}$and attain $u_{x}=c_{s}$ at the sheath edge. The angle between $\mathbf{E}$ and $\mathbf{B}$ increases with $\psi$ as well, resulting in stronger drifts in the $\mathbf{E} \times \mathbf{B}$, which may compensate for the $u_{x y}-u_{\|}$disparity. 


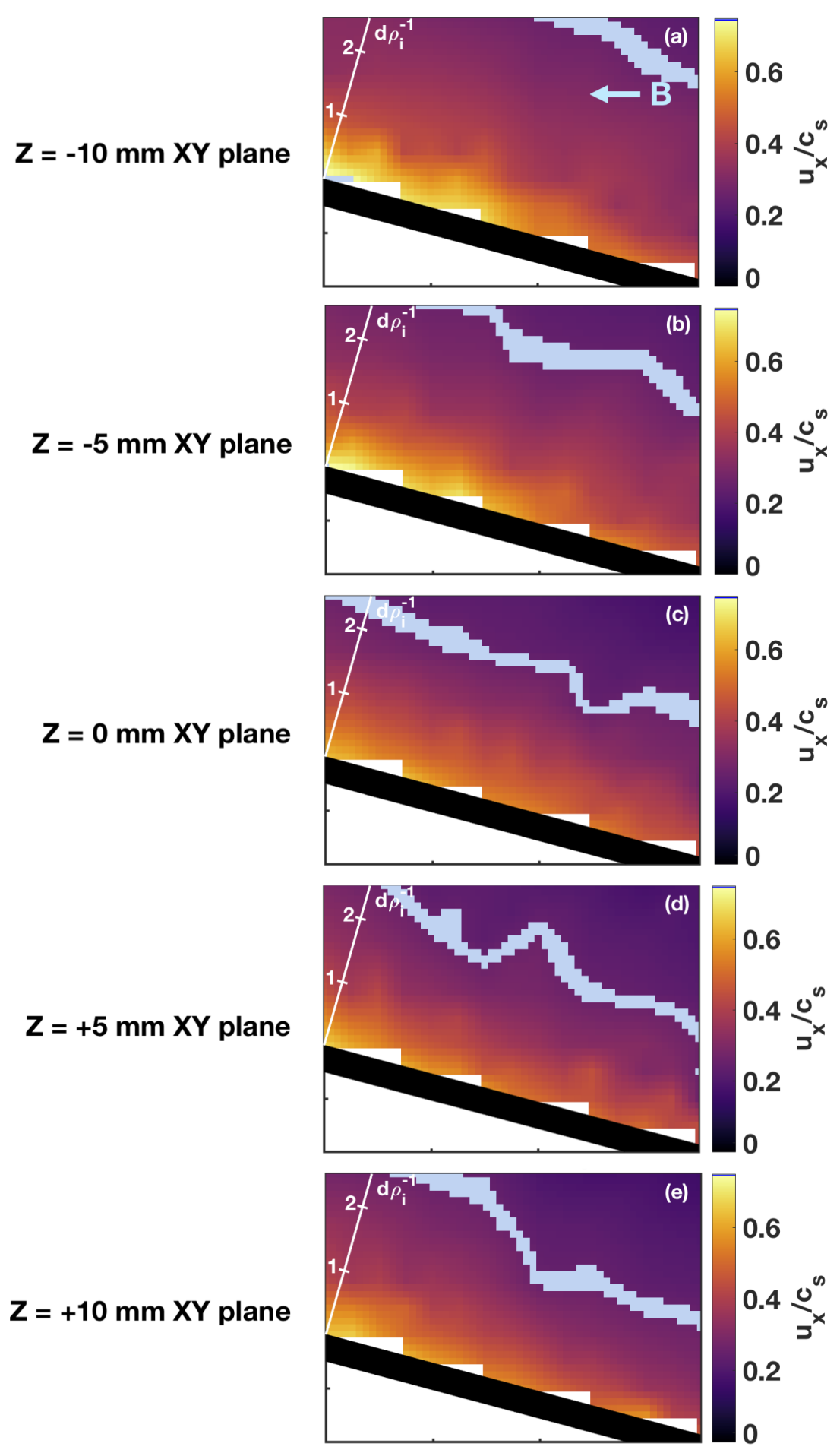

Figure 4.23: Bohm-Chodura boundary observed in 3D LIF data. Pale blue feature indicates the location where $u_{x}=c_{s} \cos (\psi) \pm 30 \mathrm{~m} / \mathrm{s}$. The location occurs at different locations for different locations in $z$. 


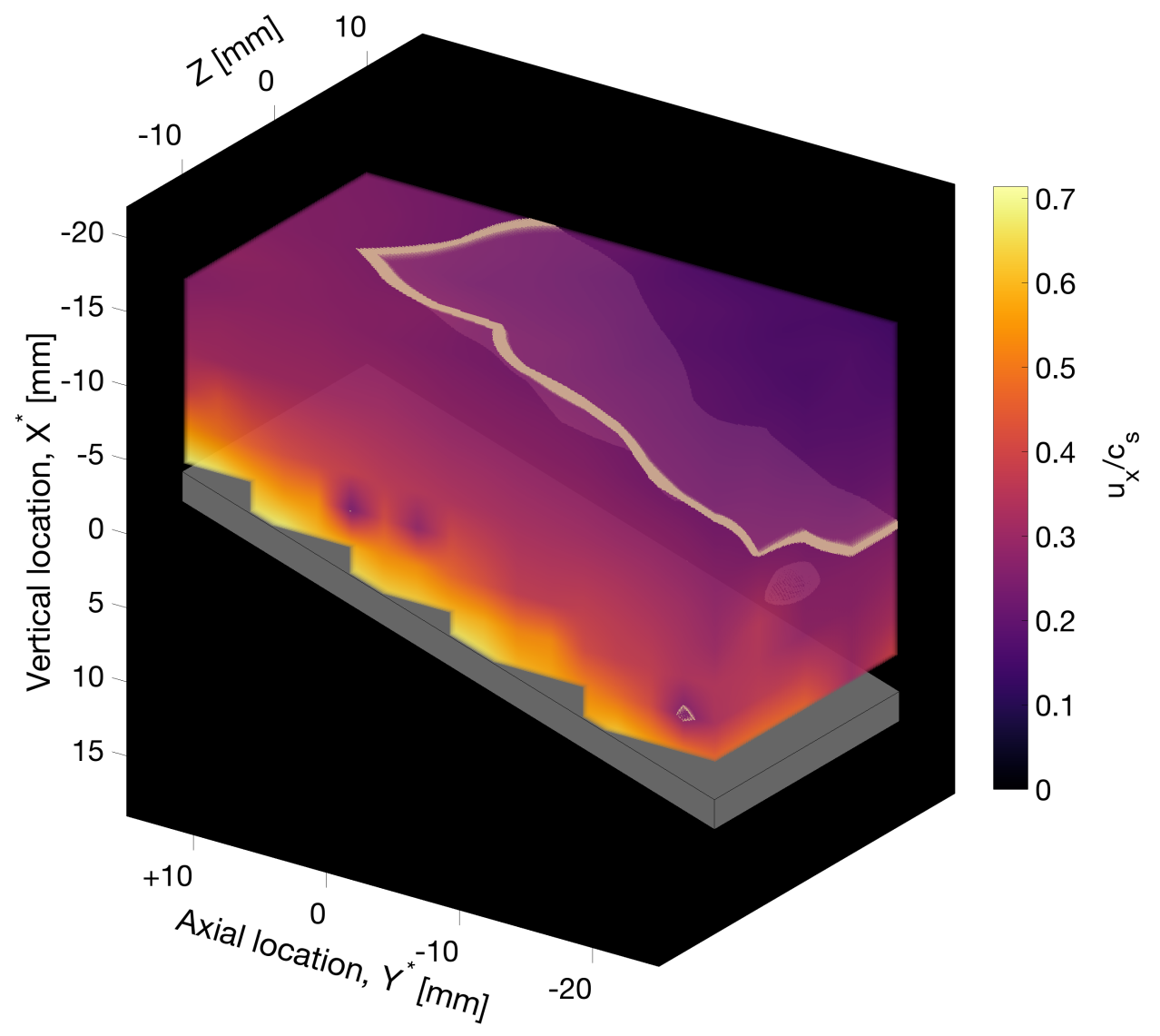

Figure 4.24: The pale manifold indicates the location where $u_{x}=c_{s} \cos (\psi) \pm 10 \mathrm{~m} / \mathrm{s}$. The criterion is met at different locations for different locations in $z$. 


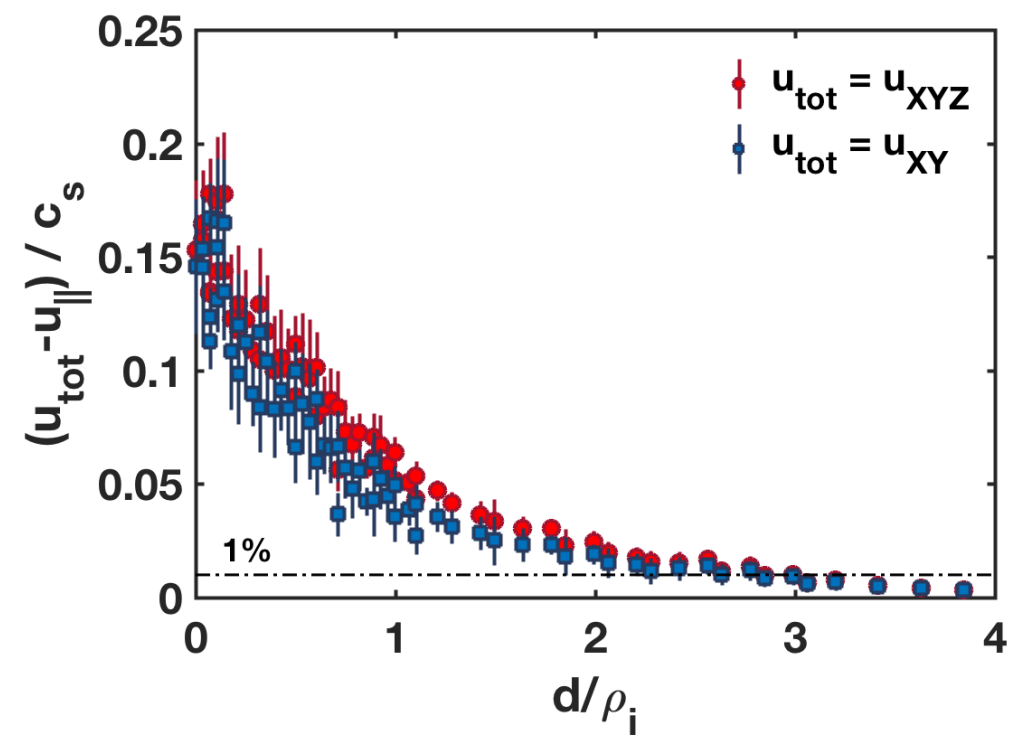

Figure 4.25: Total flow minus field-parallel flow approaching the boundary surface when $\psi=74^{\circ}$. Total drift: $u_{x y z}=\left[u_{x}^{2}+u_{y}^{2}+u_{z}^{2}\right]^{1 / 2}$. Total drift limited to the $x y$-plane: $u_{x y}=\left[u_{x}^{2}+u_{y}^{2}\right]^{1 / 2}$. 


\subsection{Comparison of HELIX boundary data with col- lisional fluid and Particle-in-Cell simulations}

A more appropriate model for the HELIX boundary should incorporate collisions and drifts in all three directions $[168,169]$. This model is recovered in Ahedo's collisional treatment when $\omega_{c i} \tau_{i} \sim 1$ and $\lambda_{D} / \rho_{i} \rightarrow 0$ (he writes " $\Lambda_{c m}=\mathcal{O}(1), \Lambda_{d m} \rightarrow 0$ ") [11]. The primary difference between Chodura's picture and this model is that there is no location where $u_{\|}=c_{s}$ and the "Chodura layer," in which ions undergo the transition from $u_{\|}=c_{s}$ to $u_{x}=c_{s}$, dissolves into a unified presheath. Within this presheath, ions experience collisions and drift effects from the presence of the magnetic field, and $\mathbf{E} \times \mathbf{B}$ drifts begin in the presheath and not at the Debye sheath edge. Little to no acceleration is expected for $u_{z}$. In the HELIX boundary measurements, at no location is $u_{\|}=c_{s}$ achieved, providing initial support for the unified presheath model.

The HELIX boundary data is compared to a collisional fluid simulation carried out by Rinat Khaziev and Davide Curreli at the University of Illinois at UrbanaChampaign. The collisional fluid model is governed by four main equations: ion continuity, with ionization; momentum balance, preserving ion pressure gradient and viscosity from collisions; the ideal gas law; and the Boltzmann distribution for electrons [164]. Explicitly, the equations are:

$$
\begin{aligned}
\nabla \cdot\left(n_{i} \mathbf{u}_{i}\right) & =n_{e} \nu_{\mathrm{IZ}} \\
n_{i} m_{i}\left(\mathbf{u}_{i} \cdot \nabla\right) \mathbf{u}_{i} & =n_{i} e\left(\mathbf{E}+\mathbf{u}_{i} \times \mathbf{B}\right)-\nabla p_{i}-n_{i} m_{i} \nu_{i} \mathbf{u}_{i}+n_{i} m_{i} \nu_{\mathrm{IN}} \mathbf{u}_{n} \\
p_{i} & =n_{i} k_{\mathrm{B}} T_{i} \\
n_{e} & =n_{o} \exp \left(-e \phi_{p} / k_{\mathrm{B}} T_{e}\right) .
\end{aligned}
$$

Eqs. (4.6)-(4.9) are numerically integrated from normalized distance to the wall, $d / \rho_{i}$, to where $u_{x} / c_{s}=1$ at the entrance of the Debye sheath. At this latter location there is a singularity in the fluid equations, and a disadvantage of the model is its inability to resolve sheath dynamics and the sheath-presheath transition. 
To supplement the fluid model, the data are compared to Particle-in-Cell (PIC) simulations, also developed by Khaziev and Curreli, in which a representative population of simulated particles is assigned to locations on a grid and moved at each time step by the calculated potentials established by the previous particle state. This process is iterated until an equilibrium condition arises. Collisions are modeled using Monte-Carlo collisions.

There are two major advantages of the PIC approach. First, no assumption is made about the particle distributions, which are allowed to be non-Maxwellian. The fluid model implicitly assumes that the particles exist in a nearly Maxwellian distribution. Second, the sheath-presheath transition and the internal sheath dynamics are resolved because no singularity exists. The major disadvantage of the PIC approach is that it is computationally expensive. As a result, the simulation space is limited. In simulating the HELIX boundary, a 1D3V model was considered with a $2.5\left(\approx 4 \rho_{i}\right)$ simulation space.

The critical importance of collisions to ion drifts in the boundary is clear from a comparison of these simulations to the data, as shown in Fig. 4.26. In (a), which shows the ion flow toward the wall, the PIC simulations that omit collisions (red) overestimate the flow of the ions. When collisions are included (yellow), they provide a mechanism for slowing the acceleration of the ions, and data and simulation agree well. The collisional fluid model also describes the data well in this direction but does not show the final acceleration of the ions in the sheath. Importantly, applying the LIF instrument response function to the simulation produces a synthetic diagnostic which smooths the sheath acceleration, matching the LIF data up to the wall surface.

The $u_{y}$ data in (b) shows good agreement with the collisional fluid and PIC simulations, even the collisionless PIC case. The flows in this direction are qualitatively described by collisionless simulations. Quantitative agreement requires the inclusion of collisions and an external force to reproduce the high initial flows observed at the experimental domain entrance. Without this force, the PIC simulations do 

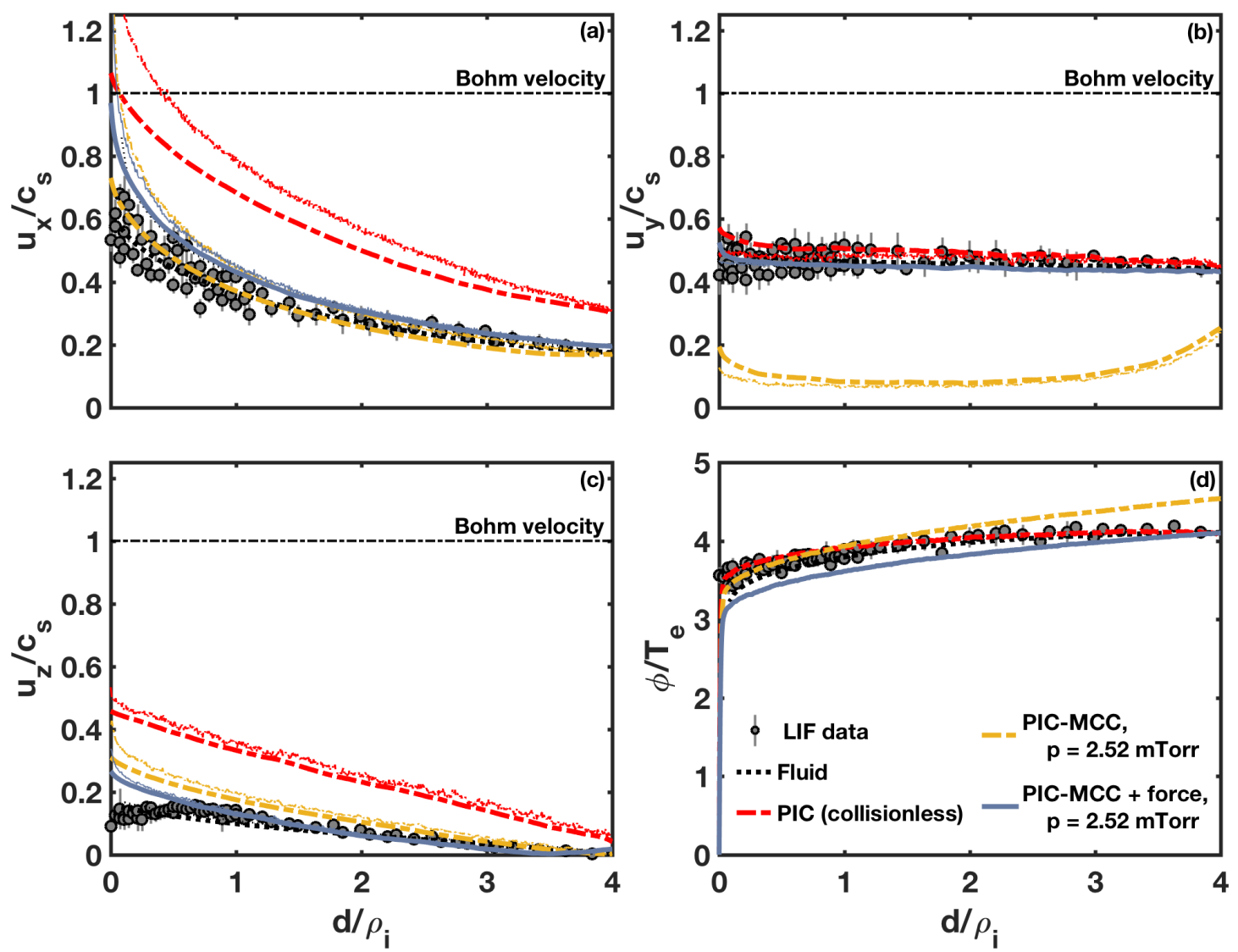

FiguRE 4.26: Comparison of data and simulations, showing three velocity dimensions and the plasma potential. Drift velocities are normalized to the local acoustic velocity $c_{s}=\sqrt{k_{\mathrm{B}}\left(T_{e}+T_{i}\right) / m_{i}}$. Plasma potential is normalized to the local electron temperature. All quantities are averaged over $z$. Thin lines represent the results of the simulation without applying synthetic diagnostics. The corresponding thick lines represent the predicted LIF data based on the sample volume geometry.

not reproduce the ion drift between the domain entrance and the wall. The nature of this force is discussed below.

The $u_{z}$ drift, predicted to occur in the $\mathbf{E} \times \mathbf{B}$ direction, is observed as a function of distance to the wall in (c). For experiments at lower $\psi$, Siddiqui et al. required ion flows in this direction of $10 \%-40 \%$ of $c_{s}$ [22]. The HELIX observations lie within that window at $18 \%$ - 20\%. Here, too, the collisional fluid and PIC models adequately capture the majority of the drift behavior, which is significantly overestimated when collisions are omitted. None of the models replicate the decrease in $u_{z}$ just above the surface. It is possible that more sophisticated definitions of the sample volume geometry would reproduce the feature if applied 

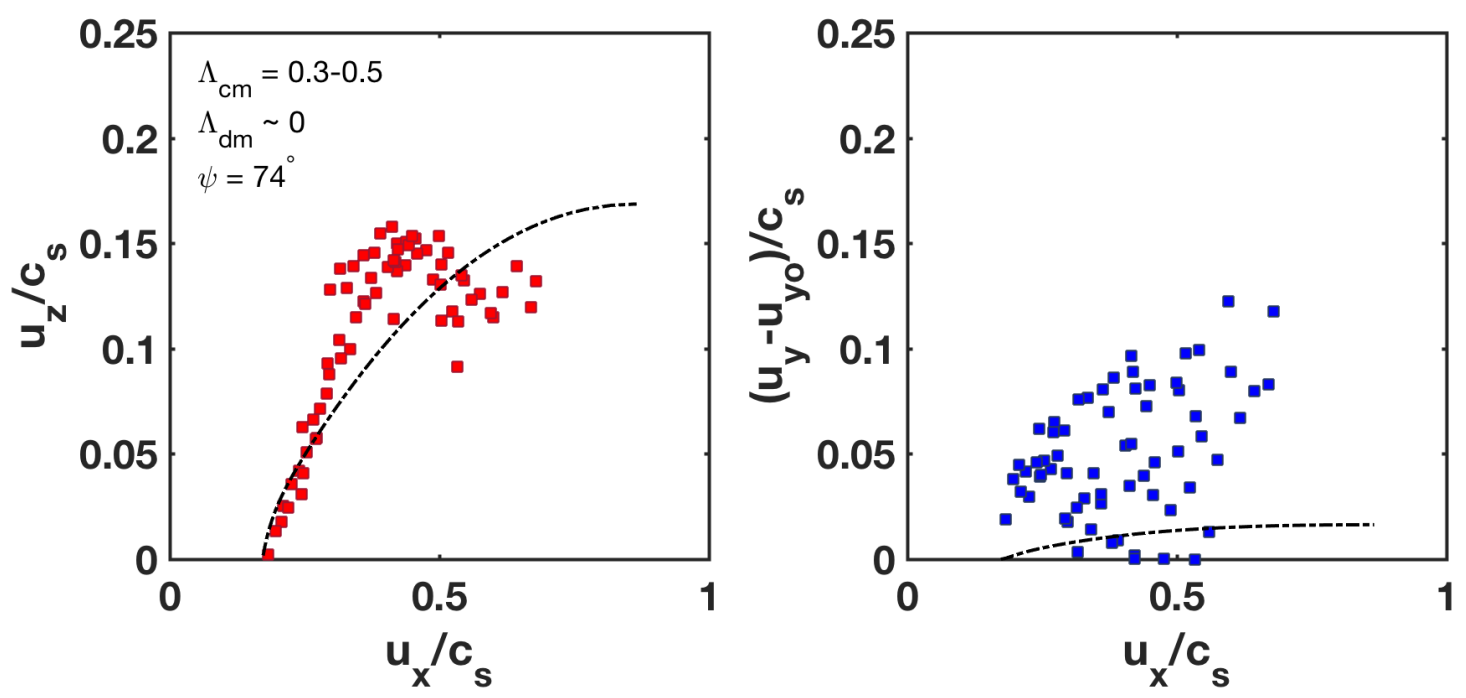

FigURE 4.27: Normalized $u_{y}$ and $u_{z}$ as functions of $u_{x}$. Data are compared to collisional fluid models (black lines).

as a simulation synthetic diagnostic. At the same time, none of the simulations include plasma-material interactions like secondary electron emission, recycling, sputtering, or particle reflection. Prof. David Ruzic at the University of Illinois at Urbana-Champaign proposed that a local cloud of recycled particles from laser heating or ion bombardment of the surface could exist within several millimeters of the surface. Such a population of slow heavy particles would present a collisional friction force to the ions, reducing their speed.

The $u_{y}$ and $u_{z}$ components are shown as a function of $u_{x}$ in Fig. 4.27. These data are compared with the fluid simulations provided by Ref. [164]. The $u_{x}$ component is offset by an initial drift because the experimental domain does not encompass the presheath entrance. Similarly, $u_{y}$ enters the domain with an initial velocity, so this velocity has been subtracted for the sake of comparison.

The fluid simulation matches the $u_{z}$ data well initially before underestimating $u_{z}$ at intermediate $u_{x}$. The agreement at $u_{x} / c_{s}>0.5$ is only due to the unexpected decrease of $u_{z}$ near the wall. Without this decrease, it appears that the fluid model would capture the qualitative behavior of the ions but would underestimate the flow magnitude. This is likely due to the high sensitivity of this model to the absolute background neutral density, which remains unknown. Likewise the 
scattered $u_{y}$ component is underestimated by the fluid model, which suffers from the same neutral density uncertainty. These plots exaggerate the disparity between the model and the data. According to the calculations reported by Ahedo, the $u_{y}$ should increase by a factor of 5 when $\omega_{c i} \tau_{i}=0.3 \rightarrow 3$ and the $u_{z}$ by a factor of about 50. With that level of sensitivity to the collisionality, it is remarkable that the simplified model does so well. Of course, the predictions of the model remain to be verified for other collisionality and magnetic angle scenarios.

Regarding the axial force accelerating the ions upstream: I argue that the $u_{y}$ ion flow is due to a large-scale effect of the helicon equilibrium and is not physics relating to magnetic boundaries. While there appears to be some initial, unobserved acceleration of the ions before they enter the experimental domain - see for example, the entrance velocity of $u_{x} / c_{s} \approx 0.2$ - there does not appear to be significant acceleration of the ions along $u_{y}$, at a slight angle to the chamber axis. There are three hypotheses for the origin of this initial acceleration. 1) There is a strong axial ion pressure gradient driving the ions to this initial speed, 2) the ions are entrained in a strong background neutral stream, 3) there is an upstream electric potential gradient accelerating the ions toward the boundary. Of course, the force may be caused by a combination of these effects. The required force to accelerate the ions from $110 \mathrm{~m} / \mathrm{s}$ to approximately $1500 \mathrm{~m} / \mathrm{s}$ is estimated to be $F=1-2 \times 10^{-17} \mathrm{~N}$.

Probe access is limited at the test point $300 \mathrm{~mm}$ upstream of the boundary, so the ion density there is not known. The density at the domain entrance is known, along with $T_{i}$ in both locations, obtained by LIF: the upstream $T_{i}^{\text {up }}=0.27 \mathrm{eV}$, domain entrance $T_{i}^{\text {de }}=0.37 \mathrm{eV}$, and downstream density is $n_{i}^{\text {de }} \approx 10^{17} \mathrm{~m}^{-3}$. Setting the maximum upstream density to be $10^{19} \mathrm{~m}^{-3}$ sets an upper limit on the upstream pressure gradient force at $F_{i}^{p, \text { up }}=1.4 \times 10^{-17} \mathrm{~N}$. This force is sufficient to account for the initial acceleration, although a 100 -fold increase in density over the intervening distance seems unlikely. A more conservative estimate is $n_{i}^{\text {up }} \approx 10^{18}$ $\mathrm{m}^{-3}$, which corresponds to a pressure gradient that produces only $10 \%$ of the required force. 


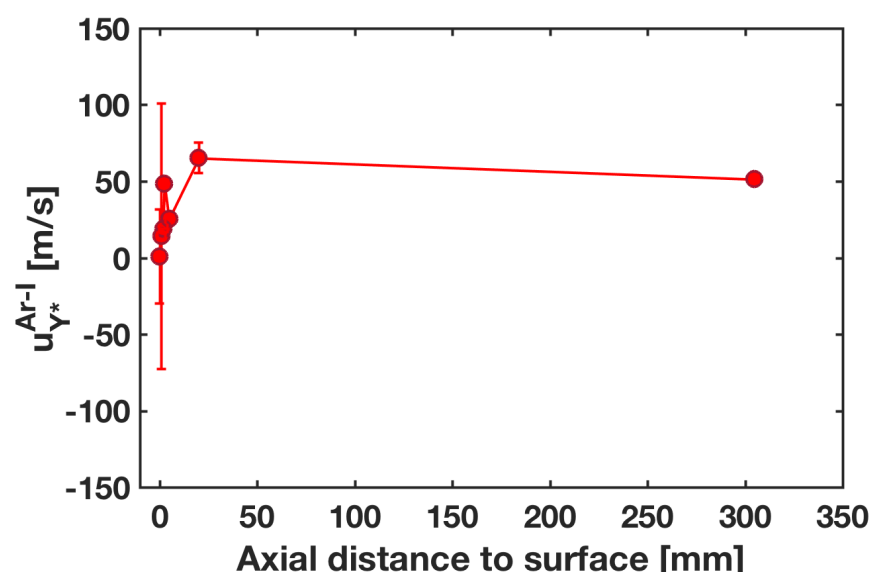

FIgURE 4.28: Mean axial flow of $1 s_{5}$ metastable Ar-I measured with LIF.

Very little external force is required if the ions are entrained in a background neutral stream, since the ion-neutral interaction is expected to be high. This hypothesis is testable using metastable Ar-I LIF. Axial $\left(u_{y^{*}}\right)$ neutral flow measurements as a function of distance to the wall are shown in Fig. 4.28. These measurements set a conservative upper bound on the axial flow of the neutral population at approximately $100 \mathrm{~m} / \mathrm{s}$, far too slow to account for the drift speed of the ions in this direction.

An axial electric field that generates a force of $F_{i}^{E}=(150 \pm 50) \times 10^{-17} \mathrm{~N}$ is required to be $E_{\|} \approx 125 \mathrm{~V} / \mathrm{m}$. Unfortunately, the electric field is not measured in the interval between the upstream test point and the boundary. Larger scale measurement near conducting boundaries were conducted in MARIA and serve as an order-of-magnitude guide. The plasma potential in those experiments, shown in Fig. 4.29, decreased $40 \mathrm{~V}$ over $20 \mathrm{~cm}$ upstream to downstream, corresponding to an coarse electric field estimate of $200 \mathrm{~V} / \mathrm{m}$. If a similar field existed in HELIX, it would be sufficient to account for the accelerated ions streaming into the boundary.

\subsection{Ion-neutral collisions in the presheath}

The ion drift velocity dependence on ion-neutral collisions is observed by varying the neutral pressure in the simulations. The results in Fig. 4.30 conform to the expectation that higher pressure leads to more ion-neutral collisions, which result 


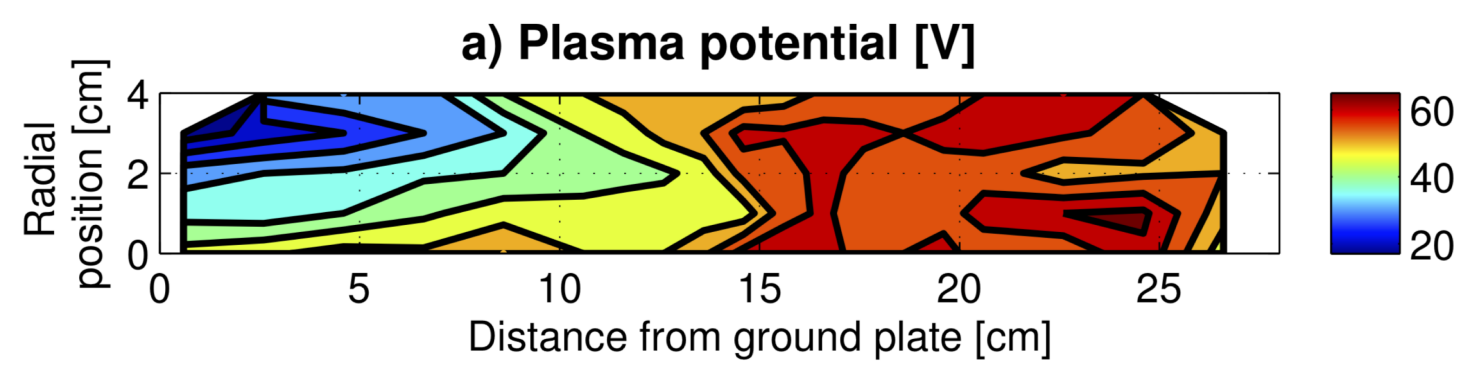

FIGURE 4.29: Large scale axial potential structure in the MARIA experiment [170]. The conducting boundary is located at $0 \mathrm{~cm}$ in this plot. Reproduced with permission from M. U. Siddiqui, Presheath and Double Layer Structures in an Argon Helicon Plasma Source (c) 2014 M. U. Siddiqui.

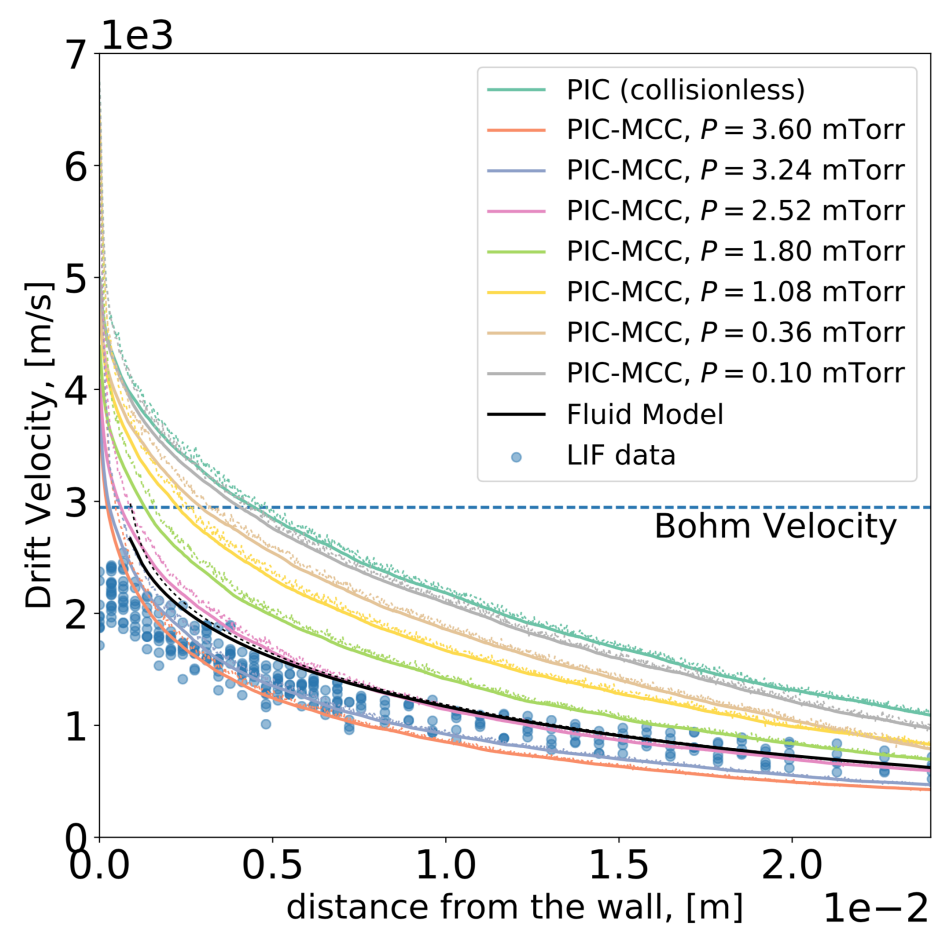

Figure 4.30: $u_{x}$ dependence on neutral pressure in simulations [164]. Thin lines are the original simulated drift velocities. Solid lines are the predicted drift velocities after applying the response function. Reproduced with permission from R. Khaziev, Multiscale Numerical simulations of the magnetized plasma sheath with massively parallel electrostatic particle-in-cell code (c) 2018 R. Khaziev.

in a decrease in the ion velocity. The ion flows with low collisionality are relatively linear with distance and accelerate significantly only at very close proximity to the surface. The higher collisionality regimes show greater than linear acceleration that begins farther from the surface. The simulated velocities describe the data best when the given neutral pressure is between 2.5 and 3.2 mTorr. This corresponds 

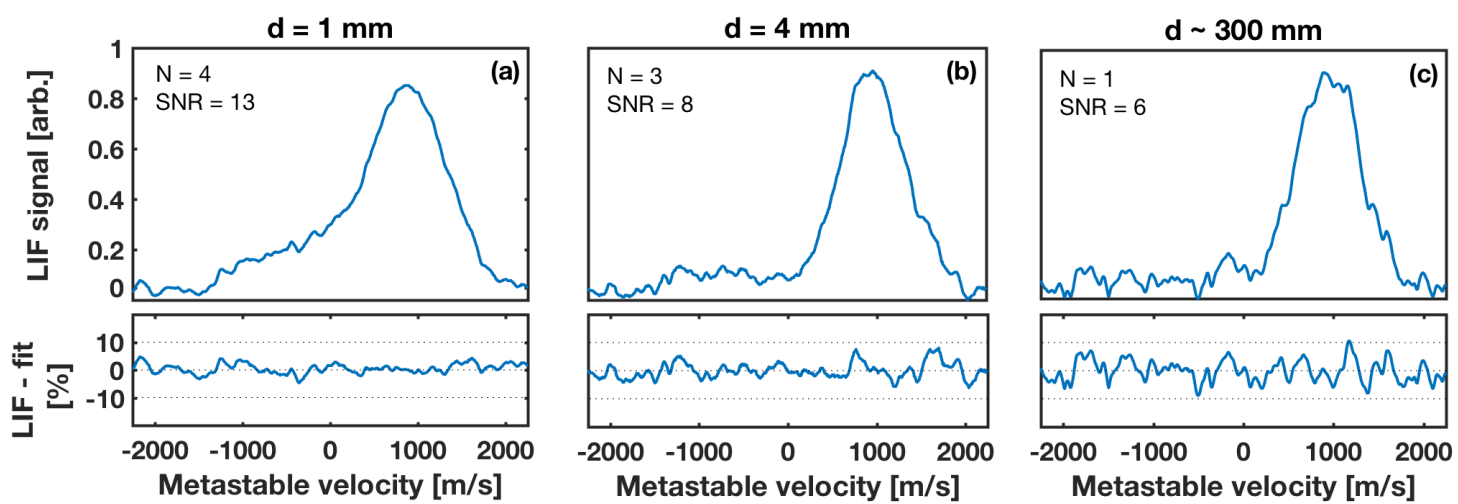

Figure 4.31: Parallel metastable Ar-I velocity distribution evolution with proximity to the wall. Each NVDF is an average of N measurements recorded at the same location and plasma parameters. The residues from bi-Maxwellian fits are shown under each spectrum. The Zeeman correction of $-895 \mathrm{~m} / \mathrm{s}$ has not been applied.

to $30 \%$ or $11 \%$ neutral depletion in the boundary, respectively.

Ion-neutral collisions such as charge exchange appear as non-Maxwellian features in the velocity distribution functions measured with LIF. When the neutral density profile is flat in $p_{n}=3.6 \mathrm{mTorr}, n_{n} \approx 10^{20} \mathrm{~m}^{-3}$, and the ion density is $n_{i} \approx 5 \times 10^{17}$ $\mathrm{m}^{-3}$. An optimistic estimate of the charge exchange signal is $n_{i} / n_{n}=0.005$, if all the ions undergo charge exchange and are measured. Even at 90\% neutral depletion, the charge exchange signal will be no greater than $5 \%$ of the spectrum maximum for these densities. An attempt to observe non-Maxwellian features in the $1 s_{5}$ metastable Ar-I population is shown in Fig. 4.31. The residues from fitting these spectra to bi-Maxwellian distributions is shown under each spectrum, with $\pm 10 \%$ levels indicated. Unfortunately, even for the $d=1 \mathrm{~mm}$ spectrum in (a), which has the best signal-to-noise ratio, the noise level is above the level of the expected charge exchange component.

On initial inspection of the spectra, a non-Maxwellian feature appears in the near vicinity of the wall at $d=1 \mathrm{~mm}$ in (a), and perhaps a small feature as well at $d=4$ $\mathrm{mm}$ in (b). In all three spectra, a bi-Maxwellian non-linear least squares algorithm finds a bulk population with a peak centered at approximately $900 \mathrm{~m} / \mathrm{s}$. Applying the Zeeman correction to this peak reveals an essentially non-flowing distribution. (The correction is $\delta u^{\text {Zeeman }}=\lambda_{12} \epsilon_{Z} B$, where $\delta u^{\text {Zeeman }}$ is the Zeeman correction, 


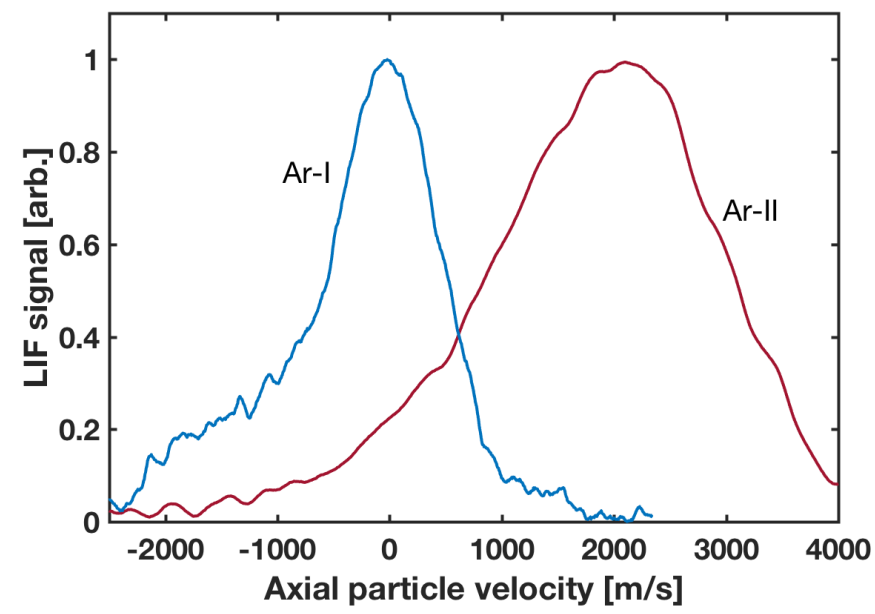

Figure 4.32: Comparison of Ar-I and Ar-II axial velocity distributions $\left(u_{y^{*}}\right)$ at close proximity to the wall. Both distributions have been corrected for the Zeeman shift.

$\lambda_{12}$ is the pump wavelength of the transition, $\epsilon_{Z}$ is the Zeeman coefficient, and $B$ is the magnetic field. For the $1 s_{5}$ transition, $\lambda_{12}=696.7352 \mathrm{~nm}$ and $\epsilon_{Z}=21.4$ $\mathrm{MHz} / \mathrm{mT}: \delta u^{\text {Zeeman }}=895 \mathrm{~m} / \mathrm{s}$.) The non-Maxwellian feature does not appear in (c), so insufficient laser polarization by the optics is excluded. In (a) and (b), the feature is at or near the expected reflection point at the inverse of the peak of the bulk population, i.e., it is mirrored about zero from the bulk spectrum. The reflection signal falls off like $1 / d^{2}$, and a small remnant may still contribute to the spectrum in (b) at the noise level. In addition to the prediction that the charge exchange contribution is very small, the ion population is moving several $\mathrm{km} / \mathrm{s}$ in the direction opposite to the direction of the "population" suggested by the non-Maxwellian feature, as shown in Fig. 4.32. (The Ar-II spectrum contains a small, $\leq 10 \%$ reflection feature near $v=0 \mathrm{~m} / \mathrm{s}$.) Any ion-neutral interactions should appear on the high-velocity side of the neutral spectrum as ions and neutral distributions exchange particles. Fortunately, these features should appear on the opposite side of $v=0 \mathrm{~m} / \mathrm{s}$ from the reflections and can be resolved. The highvelocity portion of the neutral spectrum is observed to be Maxwellian, with a maximum residue of $4.3 \%$. Ion-neutral interactions can be excluded down to this level. The best way to search for these small features is to average many spectra, ideally with the repeatability of a diode laser. Lasers that can access the $1 s_{5}$ used here to probe the metastable population exist and may be put to use in the future 


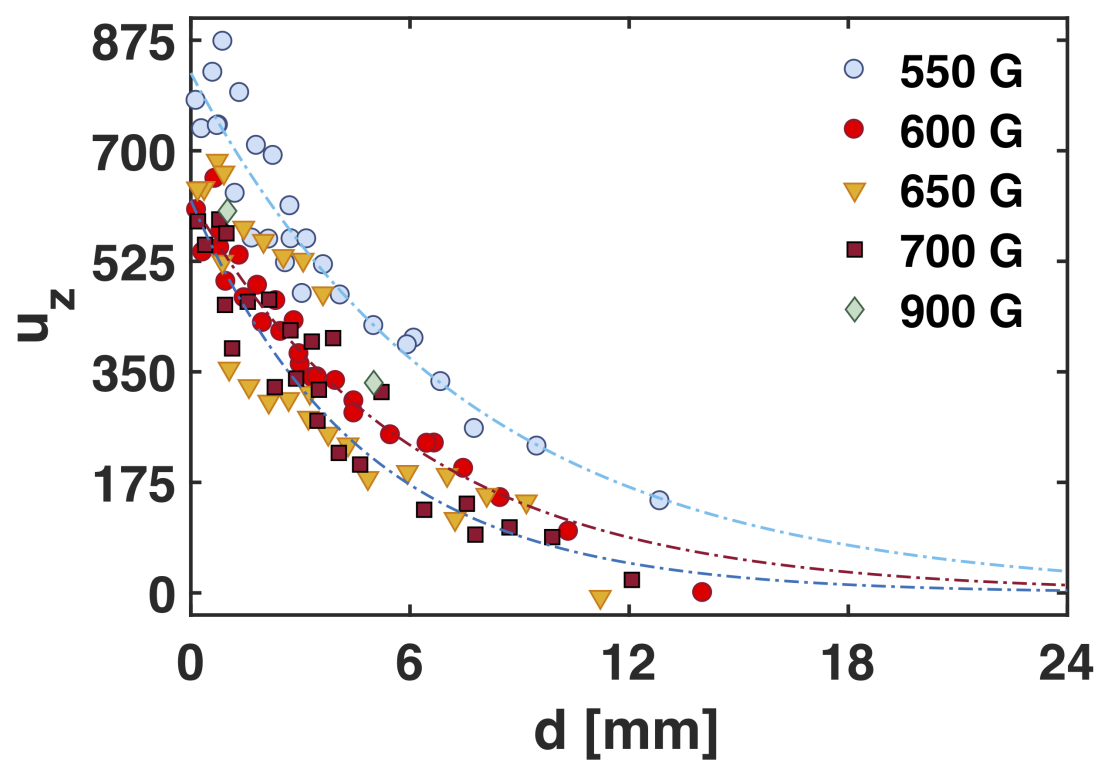

FIGURE 4.33: $u_{z}$ at several magnetic field magnitudes

for this purpose.

\subsection{Characterizing the drift in the $\mathrm{E} \times \mathrm{B}$ direction}

The flow along the $\mathbf{E} \times \mathbf{B}$ direction has a suggestive name. But is this flow an $\mathbf{E} \times \mathbf{B}$ drift or due to a different mechanism and simply "in the $\mathbf{E} \times \mathbf{B}$ direction"? The drift in the HELIX boundary was observed for a range of magnetic field magnitudes. The $u_{z}$ drift, shown in Fig. 4.33, is largest when the magnetic field is weaker than in the experiments discussed up to this point. The drift does not monotonically decrease with increasing $B$, however, and shows no clear dependence on $B$. The SNR decreases for $B>600 \mathrm{G}$, and the uncertainty dominates the difference between drifts. In these data, the drift does not decrease within several millimeters of the wall, in contrast with the data reported earlier. Whether this difference has a diagnostic origin or is due to different surface material conditions is unknown. Detailed electric field measurements in the boundary should not rely too heavily on the plasma potential derived from Langmuir probe traces. Instead, the plasma potential should be determined from an emissive probe, which was 

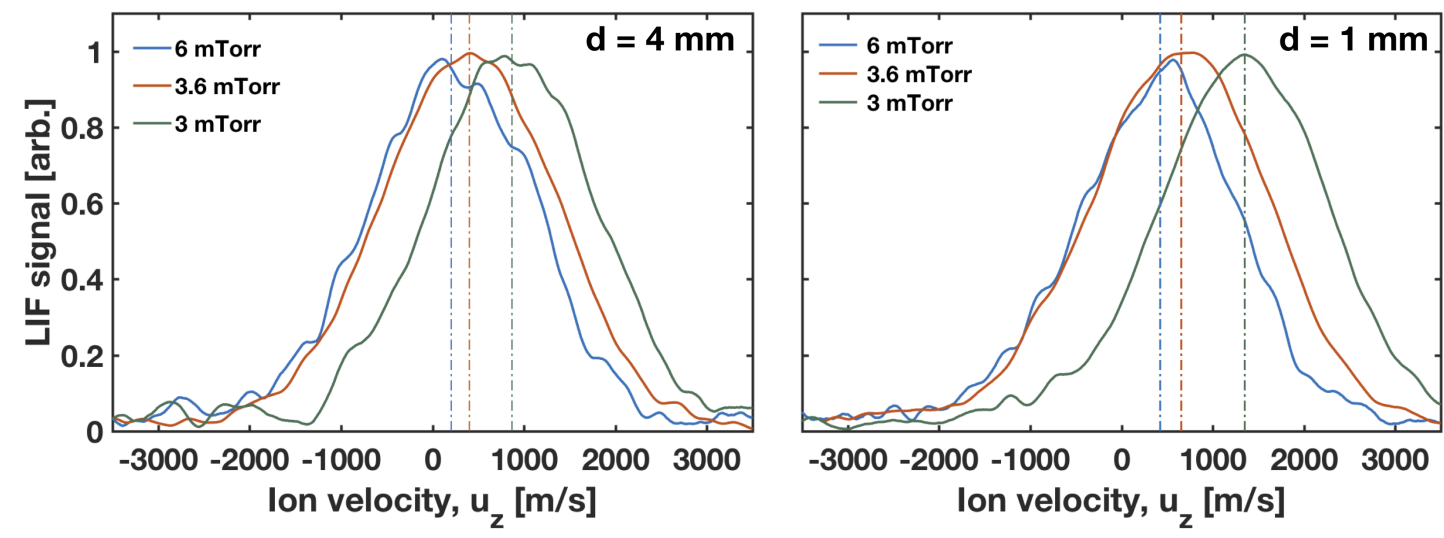

Figure 4.34: $u_{z}$ for three fill pressures and at two locations with $B=600 \mathrm{G}$.

not available at the time of these experiments. Combining accurate emissive probe measurements with low-noise LIF data over a range of magnetic field conditions should bring clarity to the dependencies of the drift in this direction.

The pressure dependence of the drift is clear from the results of varying pressure experiments shown in Fig. 4.34. At constant $B=600 \mathrm{G}, u_{z}$ increases from a low of $420 \mathrm{~m} / \mathrm{s}$ at a fill pressure of $6 \mathrm{~m}$ Torr (40 sccm gas flow rate) to $1350 \mathrm{~m} / \mathrm{s}$ at 3 mTorr (12 sccm gas flow rate). Without detailed measurements of the electric field at each pressure it is impossible to conclude what causes this velocity increase. At constant $T_{e}, \psi$, and $B$ (i.e., constant $\omega_{c i}$ ), decreasing neutral pressure increases the Hall parameter. The observation that $u_{z} / c_{s}$ increases with $\omega_{c i} \tau_{i}$ is consistent with the predictions of Ahedo for weak field scenarios. In that model, $u_{z} / c_{s}$ increases monotonically with $\omega_{c i} \tau_{i} \approx 1$.

There is more going on than can be organized according to the Hall parameter. For example, $u_{z}^{\max }$ at $B=600 \mathrm{G}$ and $p=3 \mathrm{mTorr}$ reaches $1350 \mathrm{~m} / \mathrm{s}$ while the Hall parameter for this scenario is only $15 \%$ above the Hall parameter of the main set of experiments at $B=600 \mathrm{G}$ and $p=3.6$ mTorr where $u_{z}^{\max }=$ $700 \mathrm{~m} / \mathrm{s}$. Likewise, in the $B=500 \mathrm{G}$ and $p=3.6 \mathrm{mTorr}$ scenario $\omega_{c i} \tau_{i}$ is $10 \%$ smaller, but $u_{z}^{\max }=875 \mathrm{~m} / \mathrm{s}$, exceeding the maximum flows at Hall parameters closer to the theoretical optimum. In summary, while collisional fluid models have made significant progress in describing and predicting the drift velocities in oblique 
boundaries, many questions remain, and care should be taken when relying on such simulations. 


\section{Chapter 5}

\section{Conclusions}

Plasma boundaries are one of the longest studied topics in the field and as such are too often considered an old, solved problem with little room for new investigation. On the contrary, there are sizable gaps in our understanding of the physics of plasma boundaries and the implications they hold for plasma-material interactions. The disparity between need and understanding is especially true for boundaries in the presence of magnetic fields, which will likely play a central role in the development of energy and space technologies in coming decades. A sophisticated understanding of the boundary layer when magnetic fields are present is imperative.

In this work, an investigation of a high-density plasma boundary in a moderate field ( $B=600 \mathrm{G})$ at oblique incidence to the boundary $\left(\psi=74^{\circ}\right)$ is presented. These experiments were conducted in an intermediate collisionality region in which the electrons are highly magnetized but the ions are weakly collisional $\left(\omega_{c i} \tau_{i}=0.5\right)$. These experiments build on recent two-dimensional measurements obtained at lower magnetic angle.

For the first time, the full 3D3V picture of an oblique boundary is obtained. In these experiments, several of the recent findings of Siddiqui et al. are corroborated. For example, the ions divert from their trajectories purely along the field and turn toward the plate normal. The total flow component diverts from purely parallel 
flow sooner at this more oblique higher angle. At the same time, likely due to higher collision rates, sonic or supersonic parallel flow was not observed anywhere in the experimental domain. The location of the Bohm-Chodura edge is observed in 3D as well. The criterion is met at different distances from the surface at different locations, and without clear correlation with the underlying plasma quantities, indicating the complexity of ion acceleration in the presheath.

An observation of particular importance is the previously unobserved flow in the $\mathbf{E} \times \mathbf{B}$ direction. These flows have been predicted by several authors and, if they are substantial, can change the angle of impact of particles impinging on the boundary surface, with implications for sputtering, erosion, and recycling. At fixed magnetic field, decreasing the argon fill pressure dramatically increases the drift velocity in this directions. Flows in this direction, which is parallel to the surface, introduce the possibility that the edge of the boundary may generate instabilities and become turbulent [168]. The flow in this direction is observed to attain nearly $20 \%$ of the ion acoustic speed before, surprisingly, decreasing in the near vicinity of the surface. This decrease is not represented in any of the boundary models and its underlying cause, whether physical or a diagnostic artifact, is not yet known.

Ion drift velocities were compared with predictions from fluid and Particle-in-Cell simulations. These simulations elucidated the importance of ion-neutral collisions in damping ion acceleration in the boundary region. Ion-neutral collisions, in particular, are predicted to be high. When the neutral flow is less rapid than the ion flow, these collisions cause a drag on the fast species. Markers of one kind of ion-neutral interaction, charge exchange, are sought in the velocity distributions of the essentially non-flowing (on average) neutral population. Distribution features from charge exchange interactions are expected to be small and are below the noise level of the Ar-I measurements we obtain with the current laser system (4-5\%). It is hoped that a repeatable diode laser system with very long ( $>1 \mathrm{hr}$ ) averaging will be able to extract such signals from the noise.

One result, well known to the tokamak divertor community, is the sensitivity of collisional models to background neutral density and the challenge of obtaining an 
absolute density measurement. No techniques are available for measuring absolute densities in argon. Collisional radiative models and absolute density measurements in krypton indicate a wide range of neutral depletion is possible. These ranges must be narrowed to constrain collisionality models in the boundary region.

Plasma boundary physics describes the source environment for plasma material interactions through quantities such as the ion energy angle distribution. The feedback mechanisms from the wall materials to the plasma boundary are similarly open areas of investigation and are outside the scope of the investigation here, although the material component is always in the background of the discussion. Mechanisms like secondary electron emission from the wall surface or neutral particle desorption are charge and particle source terms to the boundary region. An ideal investigation should construct a self-consistent model of the boundary, its interaction with the surface material, and the feedback of the material.

There are still many open questions regarding the plasma boundary. The multitude of scale lengths that describe the region produce a number of possible ways for the boundary to organize. Does the dissolution of the Chodura layer between $u_{\|}=c_{s}$ and $u_{x}=c_{s}$ hold for non-intermediate collisionality regimes at oblique $\psi$ ? Is there a threshold in $\omega_{c i} \tau_{i}$ or $\psi$ where this phenomenon emerges or disappears? Ahedo predicts that "when $\left[\omega_{c i} \tau_{i}\right]=\mathcal{O}(1)$ the differences between $\psi=75^{\circ}$ and $\psi=90^{\circ}$ are quite small" [11]. Is this so? What happens at grazing incidence, where the plate normal is a degree or two from being perpendicular to the field? The development of high power, high field, and highly localized laser optics will allow the investigation of these conditions. 


\section{Appendix A}

\section{Digital smoothing of signals}

\section{A.1 Overview}

To be useful for calculating distribution or probability functions, Langmuir probe current data frequently requires smoothing. Derivatives can be obtained through circuitry or by digital differentiation of the raw current. Noise is amplified with each digital derivative, producing unusable data. The problem is especially sensitive when even qualitative differences between signals can arise from artifacts of the differentiation process but are easily interpreted as underlying physics. Langmuir probe sample data is used here for demonstration, but the methods apply to any time-varying signal and are more or less effective depending on the distribution of the noise.

Several authors have recognized the importance of digital smoothing to the production of accurate EEPFs [130]. One approach is to apply a modulation voltage to the signal and retrieve the derivative from the harmonics [171, 172]. The approach applied in our experiments follows the digital smoothing methods investigated by Magnus and Gudmundsson, in which moving average, Gaussian, polynomial, Savitzky-Golay, and Blackman filtering are compared [130]. The importance of the choice of smoothing method is reinforced in that they calculate an effective electron temperature of $0.59 \mathrm{eV}$ when polynomial fitting is employed versus 1.33 
eV using a Savitzky-Golay filter. This result constitutes a factor greater than 2 difference in temperature obtained by smoothing choice alone.

For our measurements, $I_{e}^{\prime}$ and $I_{e}^{\prime \prime}$ data are prepared in a similar way: several filtered signals are created and the method that minimizes mean-squared-error (MSE) and maximizes the coefficient of determination $R^{2}$ is used for further calculations. Smoothing is optimized after each derivative. Magnus and Gudmundsson find polynomial fitting to be "unsatisfactory for all our measured data," so it is abandoned as an option here as well. The smoothing methods tested are: moving average, Gaussian, Savitzky-Golay, and Blackman.

\section{A.2 Moving average}

The moving average uses the built-in $\operatorname{smooth}()$ function in Matlab, called with the moving option. This algorithm assigns to the smoothed signal $S_{\mathrm{sm}}$ of $N=1000$ total samples, at index $n \in[1, N]$, the mean of a window of width $w_{\mathrm{MV}}$ of the original signal $S$, such that [173]

$$
S_{\mathrm{sm}}(n)=\frac{1}{w_{\mathrm{MV}}} \sum_{k=n-w_{\mathrm{MV}}+1}^{n} S(k)
$$

Distortion at the beginning and end of the trace occurs when the algorithm pads the input array with zeros for $k<1$ or $k>N$. A larger window width produces stronger smoothing but potentially more distortion; slowly varying data will contain less noise, but rapidly varying data will be reproduced with less fidelity. For probe measurements, moving averages require the greatest care in the transition region, where the current increases exponentially. Fig. A.1 shows the result of the moving average smoothing for several window widths, and Fig. A.2 shows the dependence of MSE and $R^{2}$ on window width $M$. The $R^{2}=0.9999,0.999,0.99$, and 0.9 levels are indicated by the vertical dashed line and correspond to $w_{\mathrm{MV}}=0.2$, 2.8, 6.5 and 15.3 V, respectively. Compare the difference between the ion (slowly varying) and exponential (rapidly varying) regions in Fig. A.1: the ion region is 
hardly effected by the choice in window width, while even relatively small values for $w_{\mathrm{MV}}$ produce distorted traces.
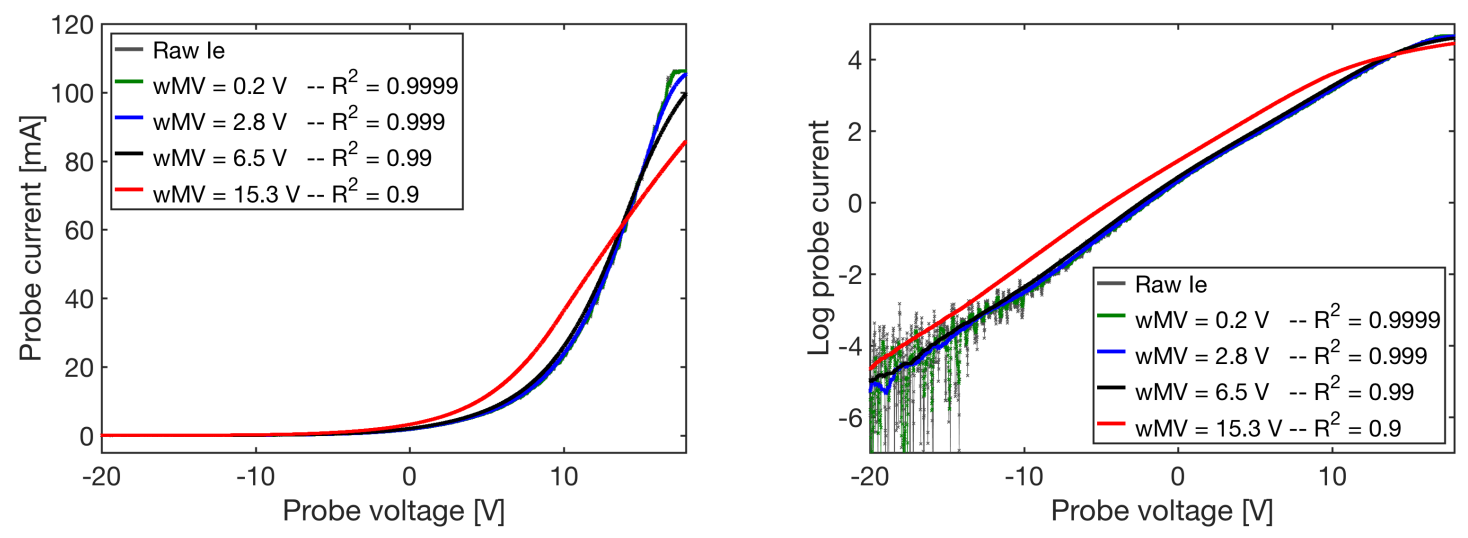

FiguRE A.1: IV\#16 smoothed by moving average using several window widths.
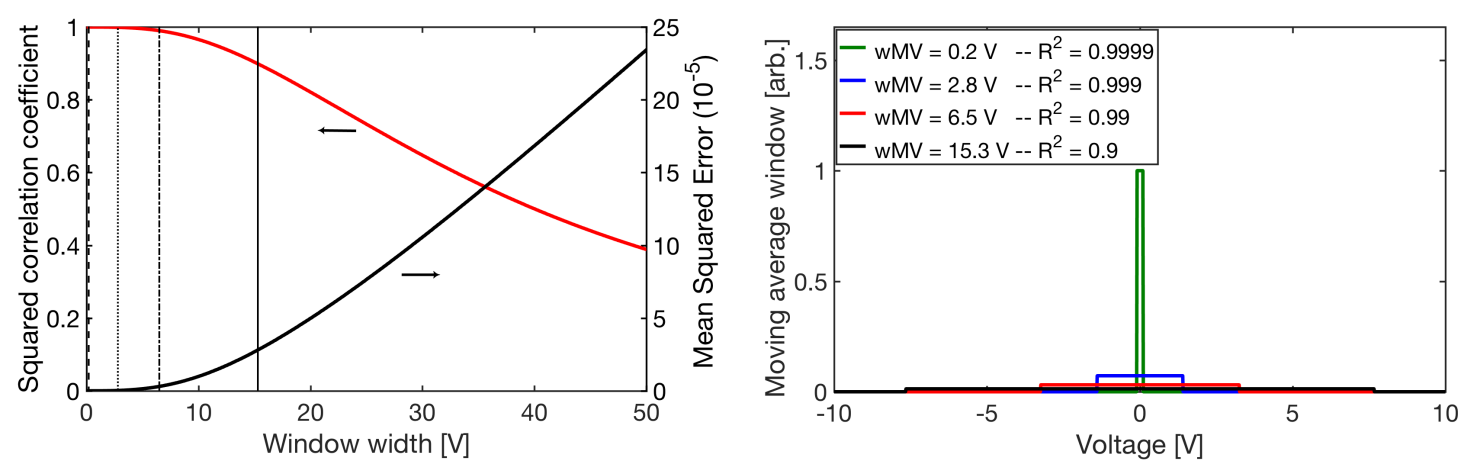

Figure A.2: Mean squared error and $R^{2}$ coefficient dependence on window width for moving average smoothing. The vertical lines indicate $R^{2}=0.9999$, $0.999,0.99$, and 0.9 , corresponding to $w_{\mathrm{MV}}=0.2,2.8,6.5$ and $15.3 \mathrm{~V}$, respectively.

\section{A.3 Gaussian convolution}

The Gaussian smoothing method convolves the signal with a smoothing window $w_{\mathrm{G}}$, generated using Matlab's built-in function gausswin(), such that

$$
w_{\mathrm{G}}(n)=\exp \left[-n^{2} / 2 \sigma^{2}\right] \quad \alpha \doteq(M-1) / 2 \sigma
$$

where $M$ is the total width of the window. The number of samples in the data record is used as an initial setting for $M$, such that $M=1000$. The Gaussian 
method does not inherently treat end effects well, so the signal is padded by concatenating an additional 1000 points equal to the first current data point to the beginning of the data stream and concatenating 1000 points equal to the last data point to the end. Without this step, the high current signal rolls off sharply. $w_{\mathrm{G}}$ is normalized by its sum to preserve the magnitude of the signal under convolution.

The Gaussian convolution method is similar to the moving average method, except that the Gaussian method strongly weights points neighboring the test point, but the points at the edges of the window are weighted weakly and contribute little. The moving method, by contrast, weights all points within the window equally. The results of smoothing the $I-V$ characteristic with Gaussian convolution are shown in Figs. A.3 and A.4. As expected, narrow Gaussian windows reproduce the raw data but do not provide much smoothing, but it is possible to make the window so broad that the characteristic spuriously flattens out.
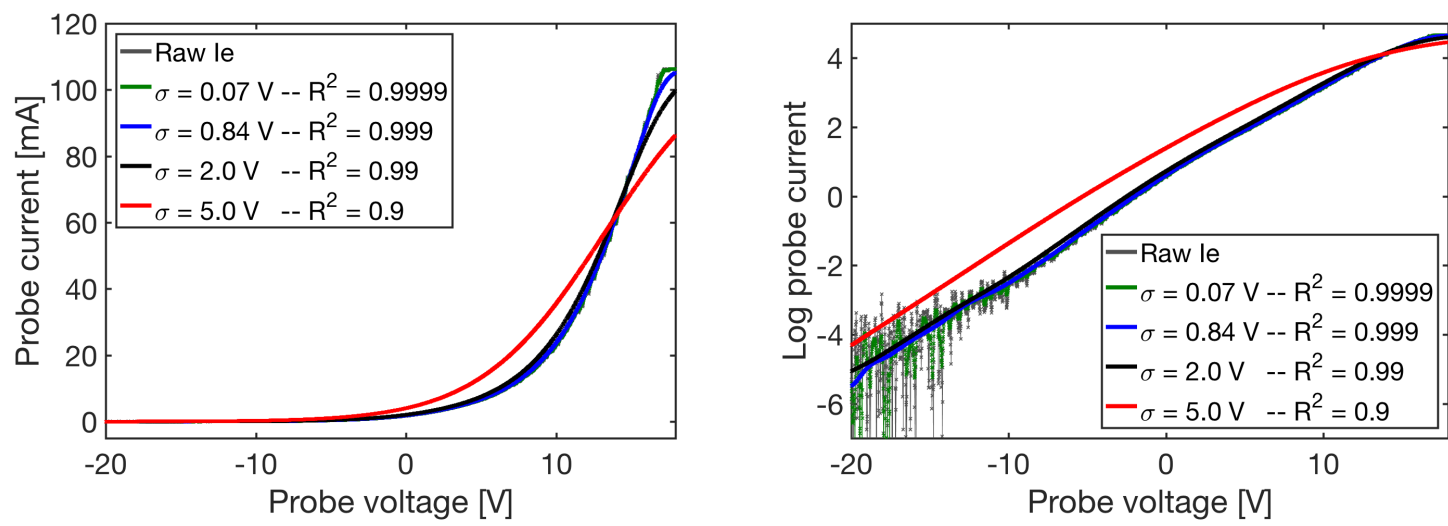

FiguRE A.3: Langmuir probe characteristic IV\#16 smoothed by Gaussian convolution for several $\sigma[V]$.

\section{A.4 Blackman window convolution}

The Blackman filter, like the Gaussian filter is convolved with noise-containing data as a smoothing technique. The advantages of Blackman window filtering were recognized for use denoising electrostatic probe data and were favored in a filter comparison by Magnus and Gudmundsson [130]. 

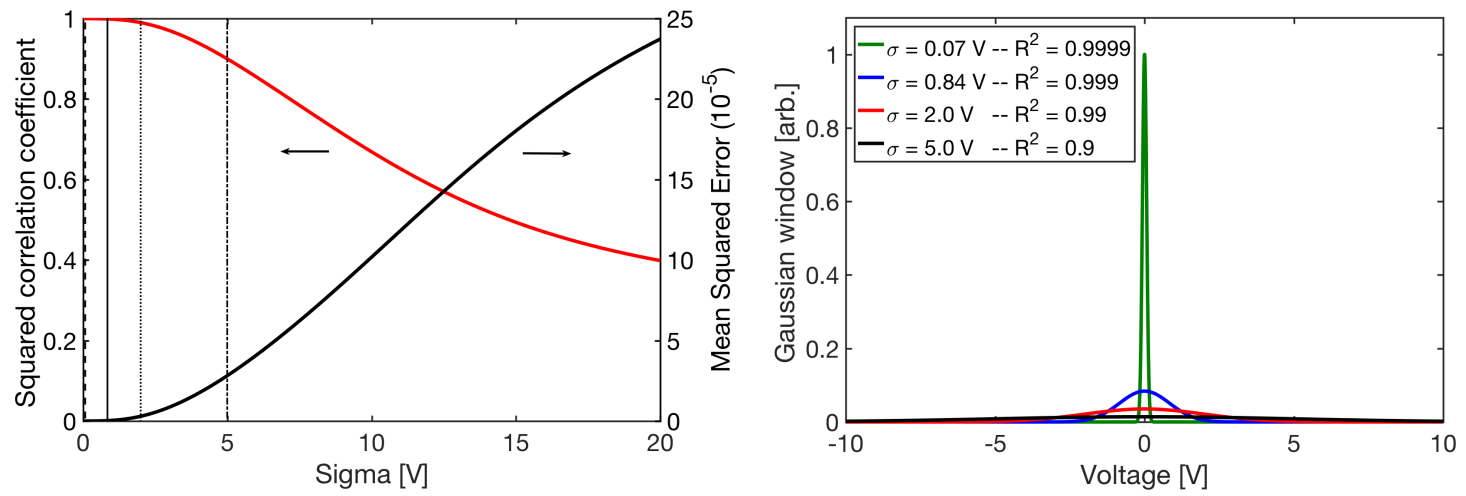

Figure A.4: Mean squared error and $R^{2}$ coefficient dependence on $\sigma$ for Gaussian convolution smoothing. The vertical line indicates $R^{2}=0.9999$, $0.999,0.99$, and 0.9 , corresponding to $\sigma=0.07,0.84,2.0$ and $5.0 \mathrm{~V}$, respectively.

The Blackman filter is generated in a similar way to the Gaussian filter, but uses the built-in blackman() function in Matlab [174, and references], with the symmetric designation, for the filter window, $w_{\mathrm{BK}}$, via the relation

$$
w_{\mathrm{BK}}(n)=0.42-0.5 \cos \frac{2 \pi n}{N-1}+0.08 \cos \frac{4 \pi n}{N-1}, \quad 0 \leq n \leq M-1 .
$$

$w_{\mathrm{BK}}$ is normalized by its sum to preserve the magnitude of the signal. The results from smoothing with a range of Blackman window widths are shown in Figs. A.5 and A.6.
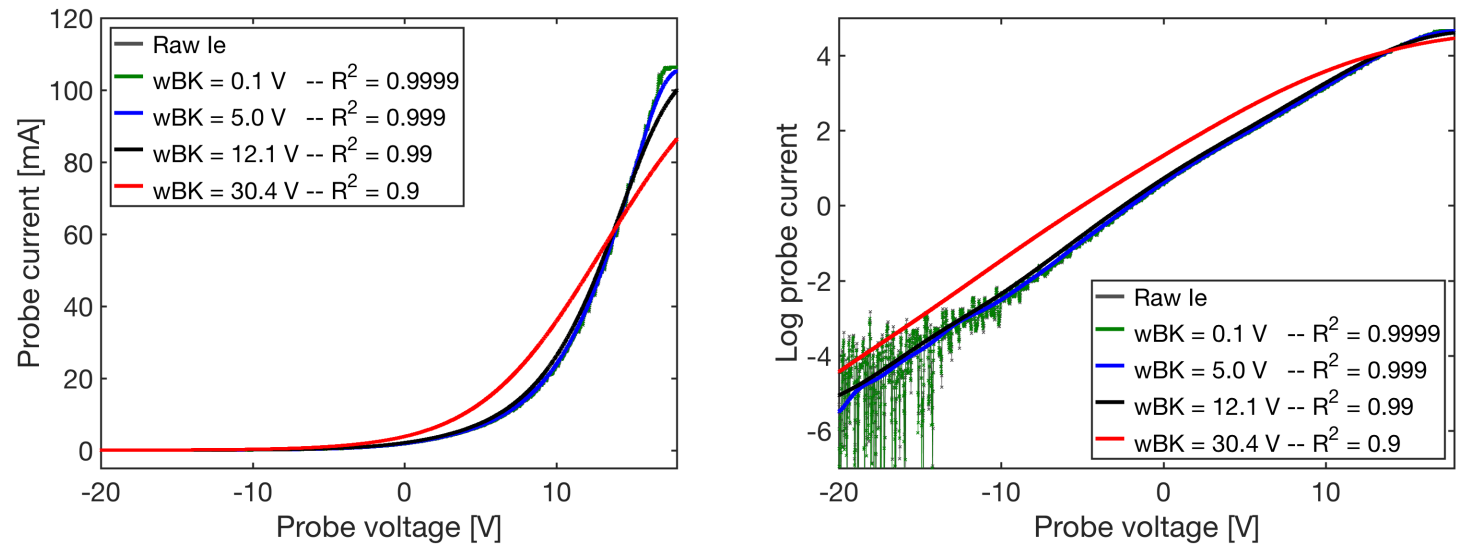

FIGURE A.5: Langmuir probe characteristic showing the same data smoothed by Blackman filter convolution for several window widths. 

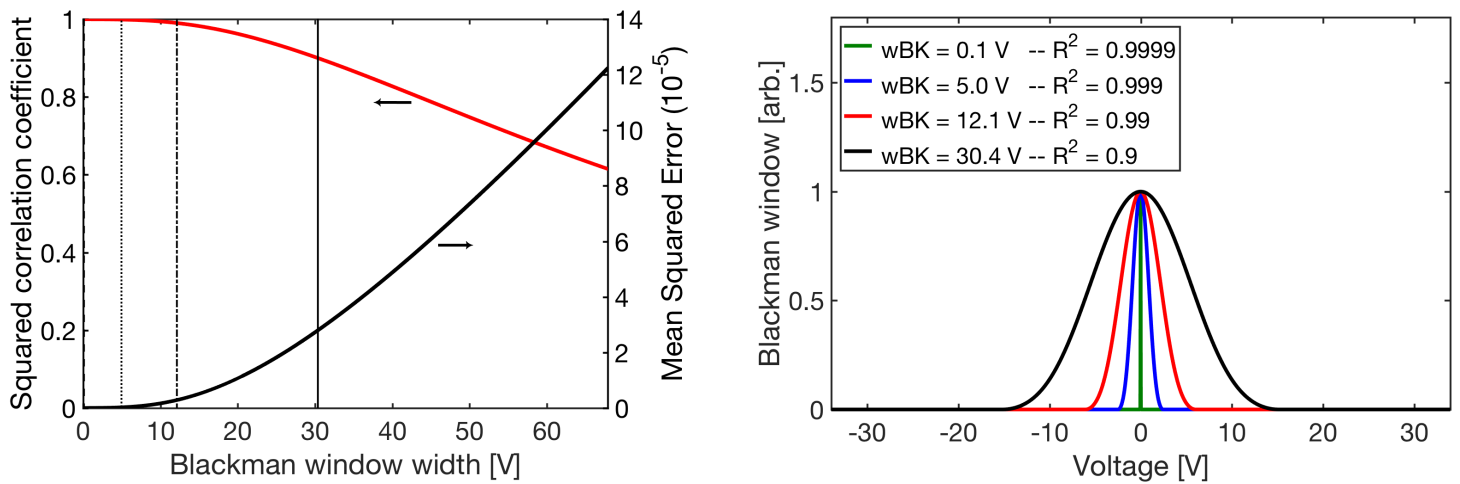

Figure A.6: Mean squared error and $R^{2}$ coefficient dependence on window width for Blackman filter convolution smoothing. The vertical lines indicate $R^{2}$ $=0.9999,0.999,0.99$, and 0.9, corresponding to $w_{\mathrm{BK}}=0.1,5.0,12.1$ and 30.4 $\mathrm{V}$, respectively. The Blackman windows are normalized to unity (at right) for clarity.

\section{A.5 Savitzky-Golay filtering}

Savitzky-Golay filtering fits a polynomial of given degree in a least-squares sense to a moving window of the signal. This filtering technique is especially flexible for signals that vary widely from short to long scales. This makes the technique attractive for use with electrostatic probe characteristics which contain rapid variation (exponential region) juxtaposed with slow variation (saturation region) that require filters able to treat both simultaneously. Savitzky-Golay filtering preserves short scale content but is often insufficient for high-noise data.

Savitzky-Golay filtering is carried out using the sgolay option of the built-in Matlab smooth() function or by convolving the data with a filtering window constructed with the sgolay() function. Similar filtering algorithms are available in other programming languages. In contrast to the previously described smoothing methods, one can select the degree of the polynomial in addition to the size of the filter window. Odd- and even-degree polynomial fitting can affect the smoothing, and it is often worth trying polynomials of several degrees before selecting the best version of the filter.

The Savitzky-Golay filter does well at the low noise levels in the transition region but diverges significantly at larger windows in noisier regions of the characteristic. 

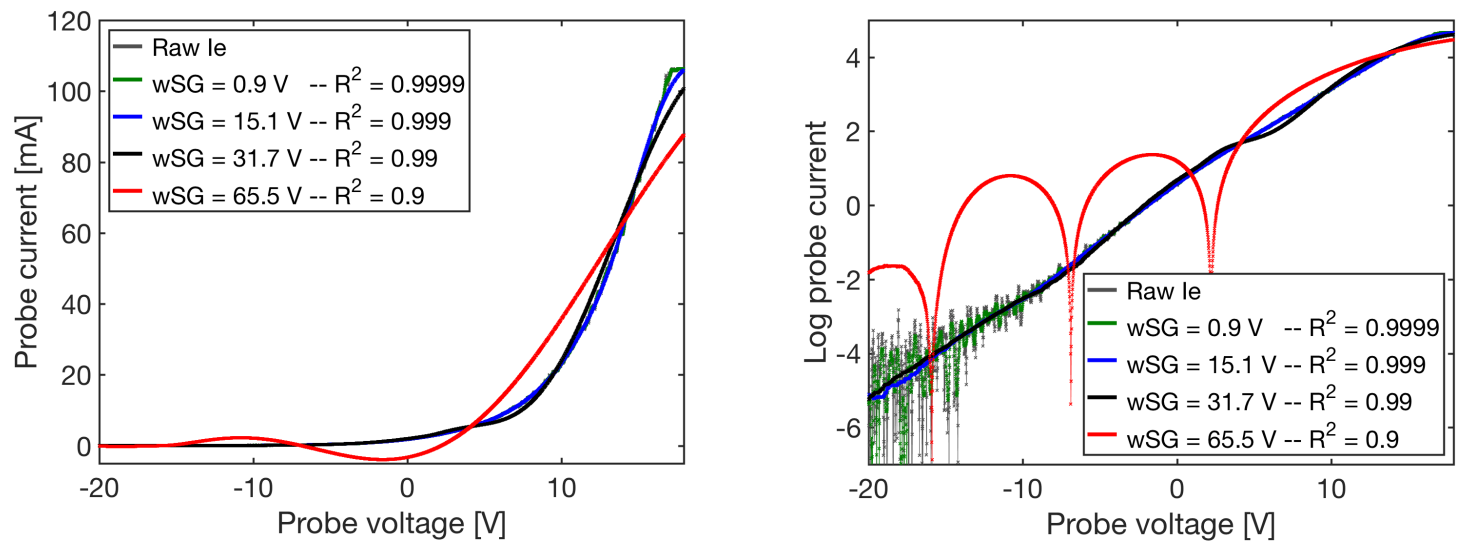

FIgURE A.7: Langmuir probe characteristic showing the IV\#16 data smoothed by Savitzky-Golay filtering for several window widths. There is no appreciable difference between 6- and 7-degree polynomial fitting for this data.

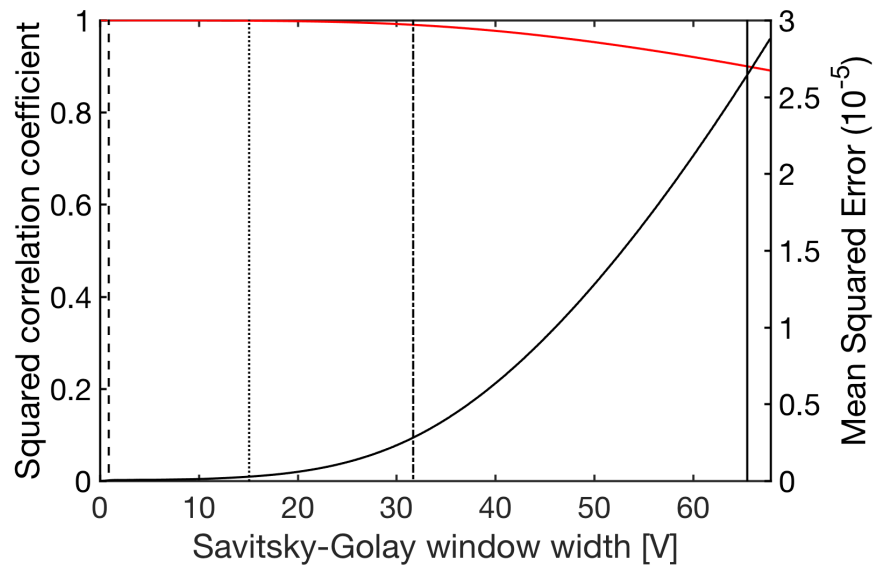

Figure A.8: Mean squared error and $R^{2}$ coefficient dependence on window width for Savitzky-Golay filtering. The vertical lines indicate $R^{2}=0.9999$, $0.999,0.99$, and 0.9 , corresponding to $w_{\mathrm{SG}}=0.9,15.1,31.7$, and $65.5 \mathrm{~V}$, respectively.

This is pronounced in the $\log$ scale characteristic in Fig. A.7 where the $w_{\mathrm{SG}}=65.5$ $\mathrm{V}$ is clearly inappropriate.

\section{A.6 Summary of results}

To emphasize: the smoothing process must be repeated at each step of the analysis of an $I-V$ characteristic. That is, once the electron current $I_{e}$ is isolated, it should be optimally smoothed so that negligible error is introduced to the measurement $T_{e}$. The smoothing optimization is repeated for $I_{e}^{\prime}$, and again for $I_{e}^{\prime \prime}$, so that the 


\begin{tabular}{|c|c|c|c|c|}
\hline Smoothing method & $R^{2}=0.9999$ & $R^{2}=0.999$ & $R^{2}=0.99$ & $R^{2}=0.9$ \\
\hline $\begin{array}{l}\text { Moving average } \\
\text { Window }[\mathrm{V}] \\
M S E\left[\mathrm{~A}^{2}\right]\end{array}$ & $\begin{array}{c}0.2 \\
3.4 \times 10^{-8}\end{array}$ & $\begin{array}{c}2.8 \\
2.7 \times 10^{-7}\end{array}$ & $\begin{array}{c}6.5 \\
2.9 \times 10^{-6}\end{array}$ & $\begin{array}{c}15.3 \\
2.8 \times 10^{-5}\end{array}$ \\
\hline $\begin{array}{l}\text { Gaussian }(2 \sigma) \\
\text { Window }[\mathrm{V}] \\
M S E\left[\mathrm{~A}^{2}\right]\end{array}$ & $\begin{array}{c}0.14 \\
2.6 \times 10^{-8}\end{array}$ & $\begin{array}{c}1.68 \\
2.9 \times 10^{-7}\end{array}$ & $\begin{array}{c}4.0 \\
3.0 \times 10^{-6}\end{array}$ & $\begin{array}{c}10.0 \\
2.8 \times 10^{-5}\end{array}$ \\
\hline $\begin{array}{l}\text { Blackman } \\
\text { Window }[\mathrm{V}] \\
M S E\left[\mathrm{~A}^{2}\right]\end{array}$ & $\begin{array}{c}0.1 \\
3.4 \times 10^{-8}\end{array}$ & $\begin{array}{c}5.0 \\
2.7 \times 10^{-7}\end{array}$ & $\begin{array}{c}12.1 \\
2.9 \times 10^{-6}\end{array}$ & $\begin{array}{c}30.4 \\
2.8 \times 10^{-5}\end{array}$ \\
\hline $\begin{array}{l}\text { Savitzky-Golay } \\
\text { Window }[\mathrm{V}] \\
M S E\left[\mathrm{~A}^{2}\right]\end{array}$ & $\begin{array}{c}0.9 \\
3.2 \times 10^{-8}\end{array}$ & $\begin{array}{c}15.1 \\
2.7 \times 10^{-7}\end{array}$ & $\begin{array}{c}31.7 \\
2.8 \times 10^{-6}\end{array}$ & $\begin{array}{c}65.5 \\
2.6 \times 10^{-5}\end{array}$ \\
\hline
\end{tabular}

TABLE A.1: Summary of digital smoothing techniques showing $R^{2}$ and MSE.

final EEPF built from $f_{p} \propto I_{e}^{\prime \prime}$ is sufficiently denoised and maintains fidelity to the original data. From the examples in Figs. A.1-A.7, it is clear that large errors can be introduced in the smoothing process.

Tab. A.1 contains a summary of these four smoothing techniques. Magnus and Gudmundsson do not suggest a general metric for comparing smoothing methods, except to say that a good smoothing function will maximize $R^{2}$ and minimize MSE, while keeping bias — signal distortion — as low as possible. In boundary experiments, there is likely to be interesting physics in those signals that exhibit non-Maxwellian characteristics, and it is best to avoid minimizing those features by a priori optimizing the data against a Maxwellian model. Review of Figs. A.1-A.7 suggests that even at the $R^{2}=0.99$ level unacceptable distortion of the original data occurs; this effect is most clearly seen in the log scale plots. At the other extreme, the $R^{2}=0.9999$ of each method leaves the data insufficiently smoothed, with a significant amount of the original noise still apparent in the processed signal. A compromise is to select the $R^{2}=0.999$ level and afterward determine the sensitivity of physical quantities to this choice.

Different signals respond differently to different smoothing method. For this reason, the Langmuir probe analysis schemes employed in Ch. 3 and 4 do a separate smoothing check at each stage of the analysis. Selection of the optimal smoothing method is chosen to minimize MSE and ensure that the introduction of signal 
distortion is avoided. Of course, smoothing does not apply to Langmuir probe data only. The hope is that the methods described in this section will help to visualize the different methods and inform the smoothing efforts of future research, whatever the diagnostic. 


\section{Appendix B}

\section{Figure Permissions}

Leaves of Grass, by Walt Whitman, is in the public domain. 
This Agreement between Dr. Derek Thompson ("You") and AIP Publishing ("AIP

Publishing") consists of your license details and the terms and conditions provided by AIP Publishing and Copyright Clearance Center.

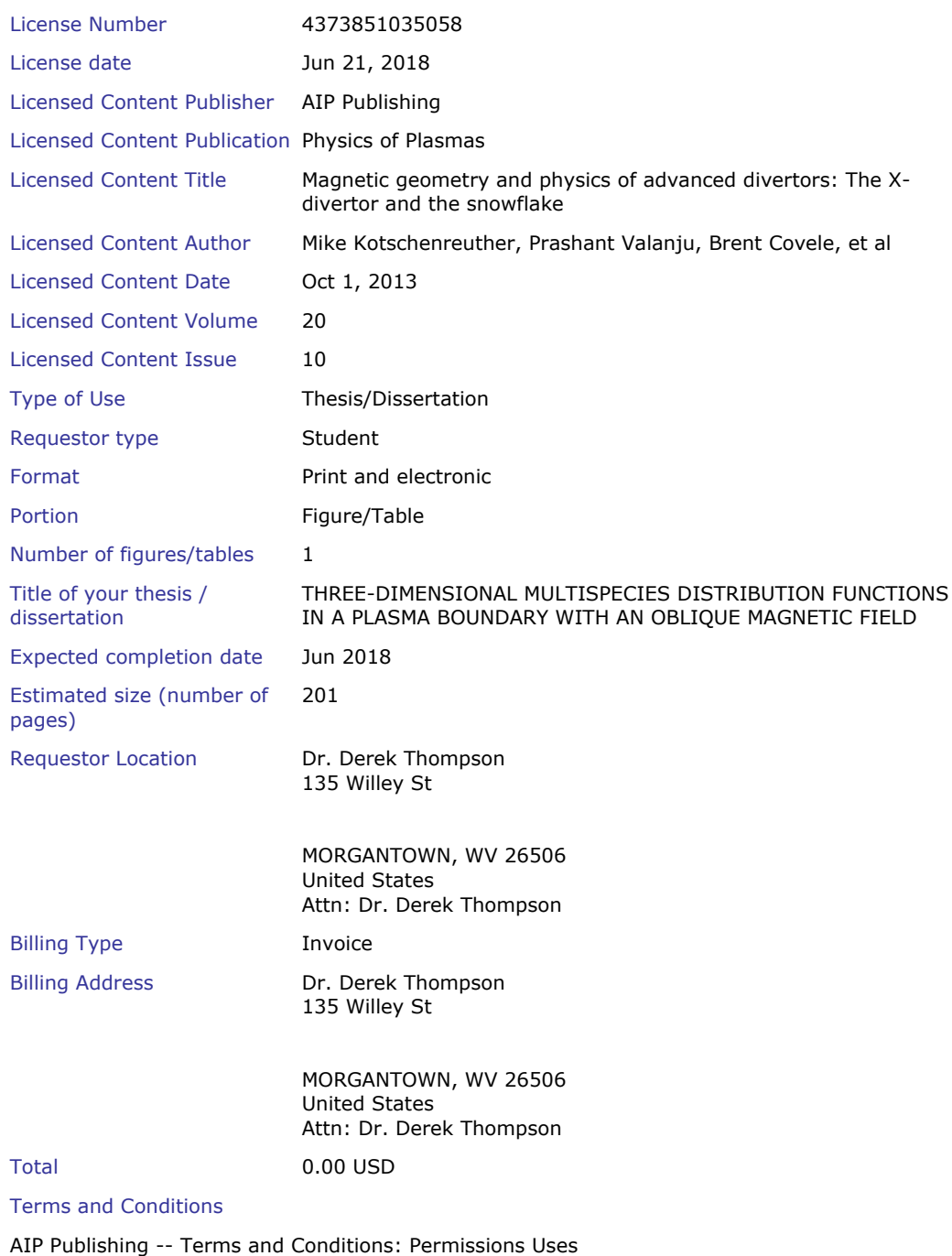


AIP Publishing hereby grants to you the non-exclusive right and license to use and/or distribute the Material according to the use specified in your order, on a one-time basis, for the specified term, with a maximum distribution equal to the number that you have ordered. Any links or other content accompanying the Material are not the subject of this license.

1. You agree to include the following copyright and permission notice with the reproduction of the Material:"Reprinted from [FULL CITATION], with the permission of AIP Publishing." For an article, the credit line and permission notice must be printed on the first page of the article or book chapter. For photographs, covers, or tables, the notice may appear with the Material, in a footnote, or in the reference list.

2. If you have licensed reuse of a figure, photograph, cover, or table, it is your responsibility to ensure that the material is original to AIP Publishing and does not contain the copyright of another entity, and that the copyright notice of the figure, photograph, cover, or table does not indicate that it was reprinted by AIP Publishing, with permission, from another source. Under no circumstances does AIP Publishing purport or intend to grant permission to reuse material to which it does not hold appropriate rights.

You may not alter or modify the Material in any manner. You may translate the Material into another language only if you have licensed translation rights. You may not use the Material for promotional purposes.

3. The foregoing license shall not take effect unless and until AIP Publishing or its agent, Copyright Clearance Center, receives the Payment in accordance with Copyright Clearance Center Billing and Payment Terms and Conditions, which are incorporated herein by reference.

4. AIP Publishing or Copyright Clearance Center may, within two business days of granting this license, revoke the license for any reason whatsoever, with a full refund payable to you. Should you violate the terms of this license at any time, AIP Publishing, or Copyright Clearance Center may revoke the license with no refund to you. Notice of such revocation will be made using the contact information provided by you. Failure to receive such notice will not nullify the revocation.

5. AIP Publishing makes no representations or warranties with respect to the Material. You agree to indemnify and hold harmless AIP Publishing, and their officers, directors, employees or agents from and against any and all claims arising out of your use of the Material other than as specifically authorized herein.

6. The permission granted herein is personal to you and is not transferable or assignable without the prior written permission of AIP Publishing. This license may not be amended except in a writing signed by the party to be charged.

7. If purchase orders, acknowledgments or check endorsements are issued on any forms containing terms and conditions which are inconsistent with these provisions, such inconsistent terms and conditions shall be of no force and effect. This document, including the CCC Billing and Payment Terms and Conditions, shall be the entire agreement between the parties relating to the subject matter hereof.

This Agreement shall be governed by and construed in accordance with the laws of the State of New York. Both parties hereby submit to the jurisdiction of the courts of New York County for purposes of resolving any disputes that may arise hereunder.

V1.2

Questions? customercare@copyright.com or +1-855-239-3415 (toll free in the US) or +1-978-646-2777. 
This Agreement between Dr. Derek Thompson ("You") and AIP Publishing ("AIP

Publishing") consists of your license details and the terms and conditions provided by AIP Publishing and Copyright Clearance Center.

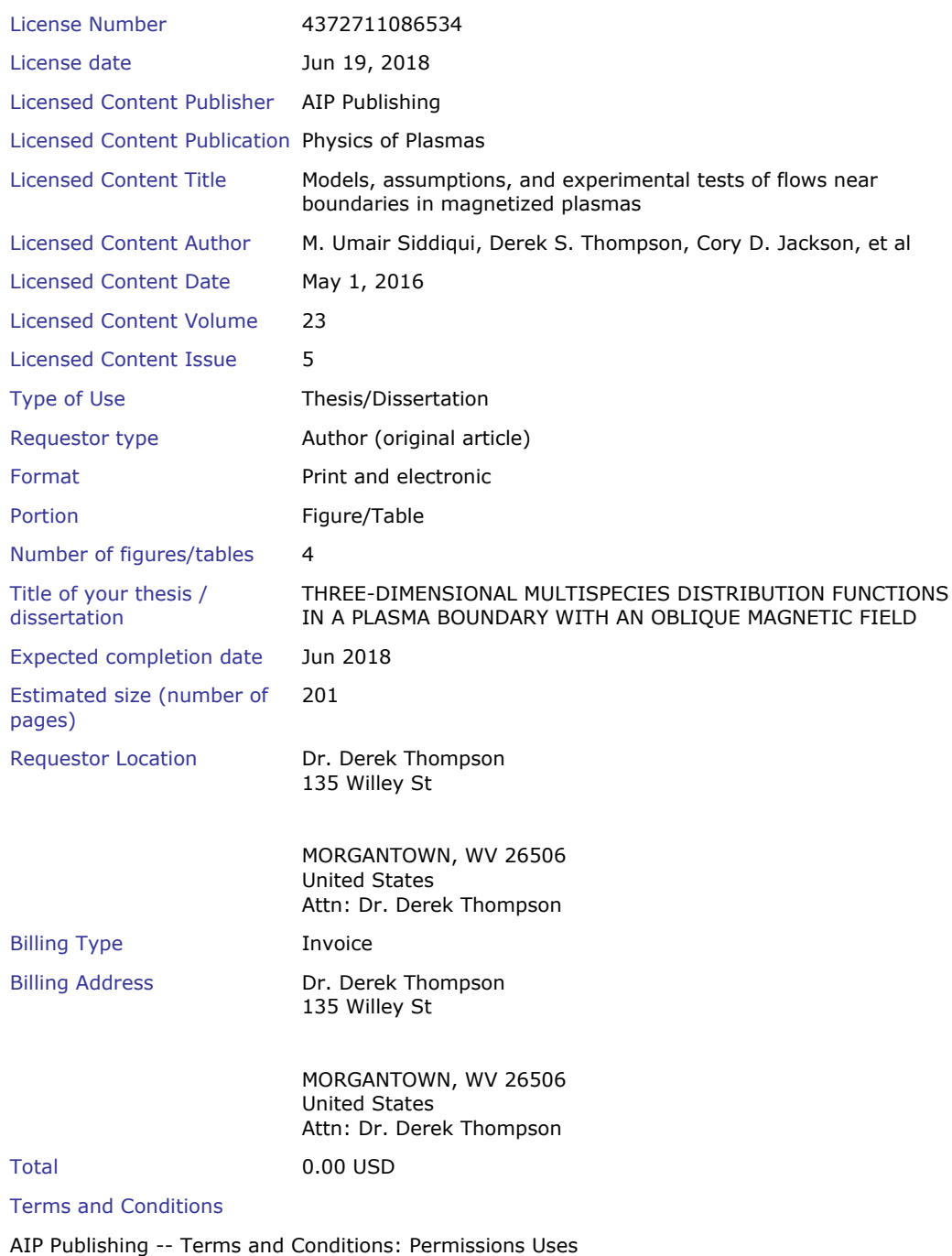


This Agreement between Dr. Derek Thompson ("You") and AIP Publishing ("AIP

Publishing") consists of your license details and the terms and conditions provided by AIP Publishing and Copyright Clearance Center.

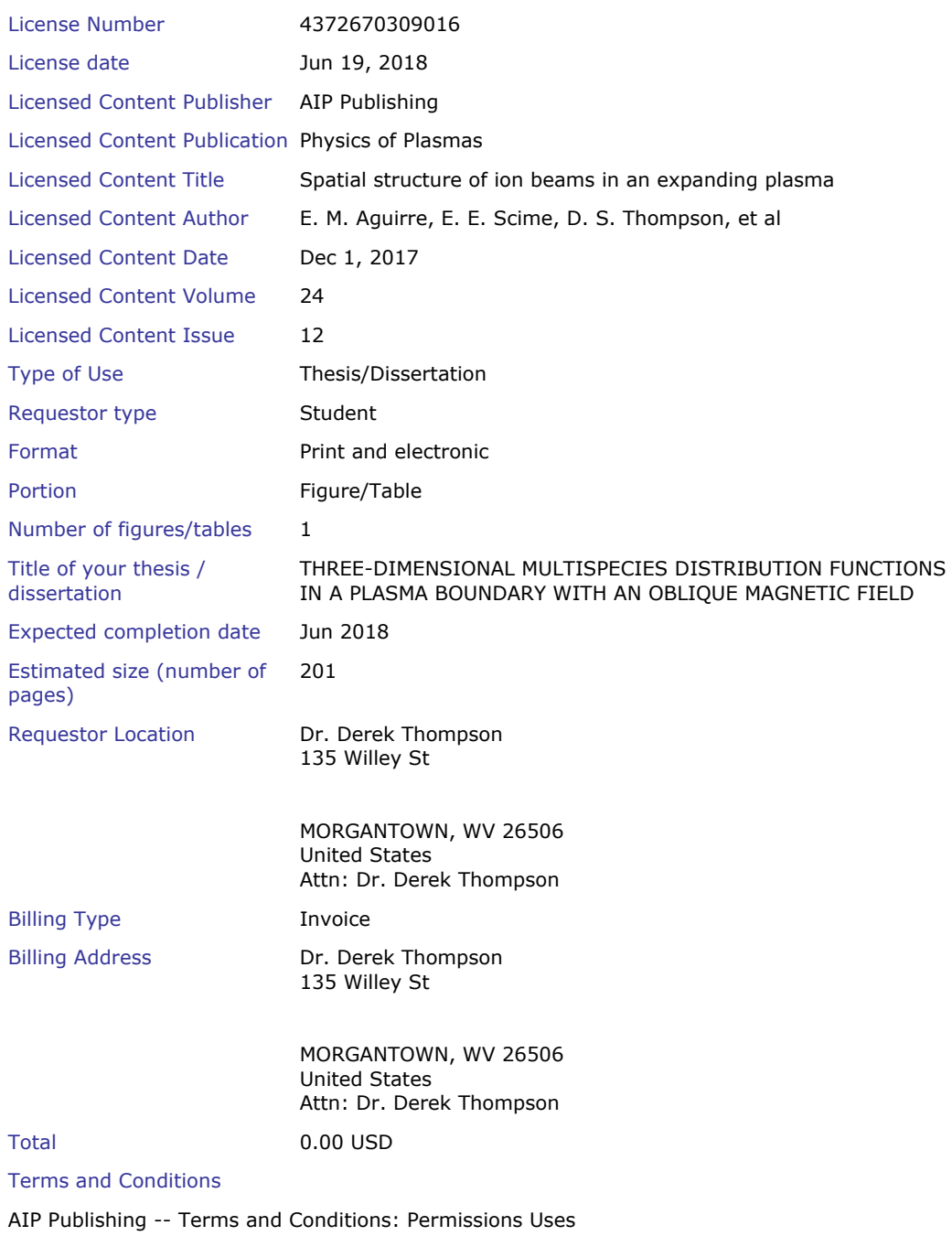

AIP Publishing -- Terms and Conditions: Permissions Uses 


\section{APS American Physical Society

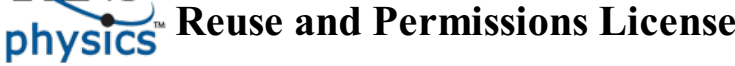

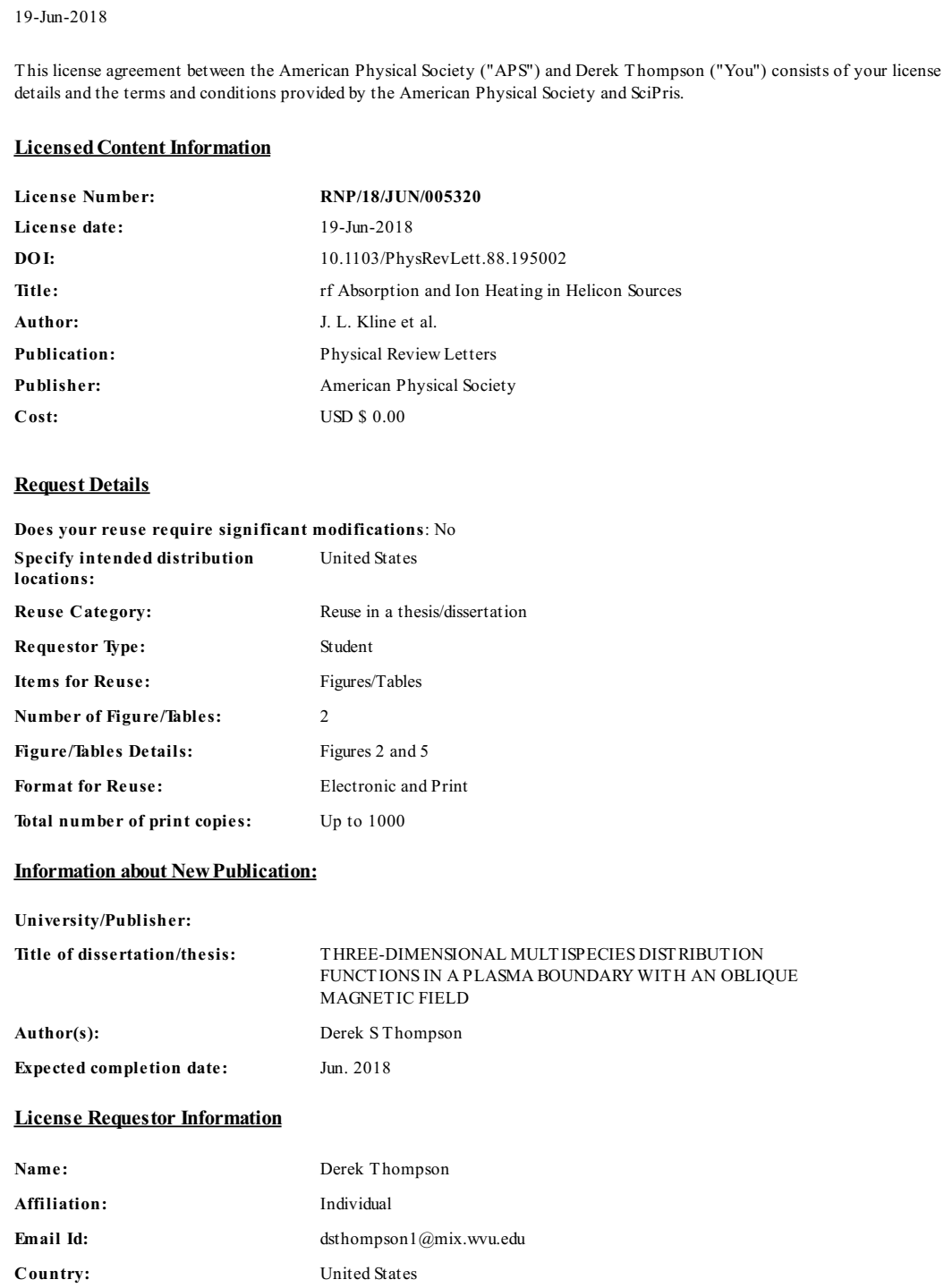

Licensed Content Information

$\begin{array}{ll}\text { License Number: } & \text { RNP/18/JUN/005320 } \\ \text { License date: } & \text { 19-Jun-2018 } \\ \text { DOI: } & \text { 10.1103/PhysRevLett.88.195002 } \\ \text { Title: } & \text { rf Absorption and Ion Heating in Helicon Sources } \\ \text { Author: } & \text { J. L. Kline et al. } \\ \text { Publication: } & \text { Physical Review Letters } \\ \text { Publisher: } & \text { American Physical Society } \\ \text { Cost: } & \text { USD } \$ 0.00\end{array}$

Request Details

Does your reuse require significant modifications: No

Specify intended distribution United States

locations:

Reuse Category: Reuse in a thesis/dissertation

Requestor Type: Student

Items for Reuse: $\quad$ Figures/Tables

Number of Figure/Tables:

Figure/Tables Details: $\quad$ Figures 2 and 5

Format for Reuse: $\quad$ Electronic and Print

Total number of print copies: $\quad$ Up to 1000

Information about New Publication:

University/Publisher:

Title of dissertation/thesis: $\quad$ THREE-DIMENSIONAL MULT ISPECIES DIST RIBUT ION FUNCT IONS IN A PLASMA BOUNDARY WITH AN OBLIQUE MAGNETIC FIELD

Author(s):

Derek S Thompson

Expected completion date:

Jun. 2018

License Reques tor Information

Name:

Affiliation:

Email Id:

Country:

Derek Thompson

Individual

dsthompson1@mix.wvu.edu

United States 


\section{APS American Physical Society physics Reuse and Permissions License}

\section{TERMS AND CONDITIONS}

The American Physical Society (APS) is pleased to grant the Requestor of this license a non-exclusive, non-transferable permission, limited to Electronic and Print format, provided all criteria out lined below are followed.

1. You must also obtain permission from at least one of the lead authors for each separate work, if you haven't done so already. The author's name and affiliation can be found on the first page of the published Article.

2. For electronic format permissions, Requestor agrees to provide a hyperlink from the reprinted APS material using the source material's DOI on the web page where the work appears. The hyperlink should use the standard DOI resolution URL, http://dx.doi.org/\{DOI\}. The hyperlink may be embedded in the copyright credit line.

3. For print format permissions, Requestor agrees to print the required copyright credit line on the first page where the material appears: "Reprinted (abstract/excerpt/figure) with permission from [(FULL REFERENCE CITAT ION) as follows: Author's Names, APS Jounat Title, Vlume Number, Page Number and Year of Publication.] Copyright (YEAR) by the American Physical Society."

4. Permission granted in this license is for a one-time use and does not include permission for any future editions, updates, databases, formats or other matters. Permission must be sought for any additional use.

5. Use of the material does not and must not imply any endorsement by APS.

6. APS does not imply, purport or intend to grant permission to reuse materials to which it does not hold copyright. It is the requestor's sole responsibility to ensure the licensed material is original to APS and does not contain the copyright of another entity, and that the copyright notice of the figure, photograph, cover or table does not indicate it was reprinted by APS with permission from another source.

7. The permission granted herein is personal to the Requestor for the use specified and is not transferable or assignable without express written permission of APS. This license may not be amended except in writing by APS.

8. You may not alter, edit or modify the material in any manner.

9. You may translate the materials only when translation rights have been granted.

10. APS is not responsible for any errors or omissions due to translation.

11. You may not use the material for promotional, sales, advertising or marketing purposes

12. The foregoing license shall not take effect unless and until APS or its agent, Aptara, receives payment in full in accordance with Aptara Billing and Payment Terms and Conditions, which are incorporated herein by reference.

13. Should the terms of this license be violated at any time, APS or Aptara may revoke the license with no refund to you and seek relief to the fullest extent of the laws of the USA. Official written notice will be made using the contact information provided with the permission request. Failure to receive such notice will not nullify revocation of the permission.

14. APS reserves all rights not specifically granted herein.

15. This document, including the Aptara Billing and Payment Terms and Conditions, shall be the entire agreement between the parties relating to the subject matter hereof. 


(4) 2 르를

$\begin{array}{ll}\text { Title: } & \begin{array}{l}\text { Formation of the Blue Core in } \\ \text { Argon Helicon Plasma }\end{array} \\ \text { Author: } & \text { Saikat Chakraborty Thakur } \\ \text { Publication: } & \text { Plasma Science, IEEE } \\ & \text { Transactions on } \\ \text { Publisher: } & \text { IEEE } \\ \text { Date: } & \text { Aug. } 2015\end{array}$

\begin{tabular}{|l|}
\hline \multicolumn{1}{|c|}{ LOGIN } \\
If you're a copyright.com \\
user, you can login to \\
RightsLink using your \\
copyright.com credentials. \\
Already a RightsLink user or \\
want to learn more?
\end{tabular}

Copyright (c) 2015, IEEE

Thesis / Dissertation Reuse

The IEEE does not require individuals working on a thesis to obtain a formal reuse license, however you may print out this statement to be used as a permission grant:

Requirements to be followed when using any portion (e.g., figure, graph, table, or textual material) of an IEEE copyrighted paper in a thesis:

1) In the case of textual material (e.g., using short quotes or referring to the work within these papers) users must give full credit to the original source (author, paper, publication) followed by the IEEE copyright line (c) 2011 IEEE.

2) In the case of illustrations or tabular material, we require that the copyright line (c) [Year of original publication] IEEE appear prominently with each reprinted figure and/or table.

3) If a substantial portion of the original paper is to be used, and if you are not the senior author, also obtain the senior author's approval.

Requirements to be followed when using an entire IEEE copyrighted paper in a thesis

1) The following IEEE copyright/ credit notice should be placed prominently in the references: (c) [year of original publication] IEEE. Reprinted, with permission, from [author names, paper title, IEEE publication title, and month/year of publication]

2) Only the accepted version of an IEEE copyrighted paper can be used when posting the paper or your thesis on-line.

3) In placing the thesis on the author's university website, please display the following message in a prominent place on the website: In reference to IEEE copyrighted material which is used with permission in this thesis, the IEEE does not endorse any of [university/educational entity's name goes here]'s products or services. Internal or personal use of this material is permitted. If interested in reprinting/republishing IEEE copyrighted material for advertising or promotional purposes or for creating new collective works for resale or redistribution, please go to http://www.ieee.org/publications standards/publications/rights/rights link.html to learn how to obtain a License from RightsLink.

If applicable, University Microfilms and/or ProQuest Library, or the Archives of Canada may supply single copies of the dissertation.

\section{BACK}

CLOSE WINDOW Copyright ( 2018 Copyright Clearance Center, Inc. All Rights Reserved. Privacy statement. Terms and Conditions. 
This Agreement between Dr. Derek Thompson ("You") and AIP Publishing ("AIP

Publishing") consists of your license details and the terms and conditions provided by AIP Publishing and Copyright Clearance Center.

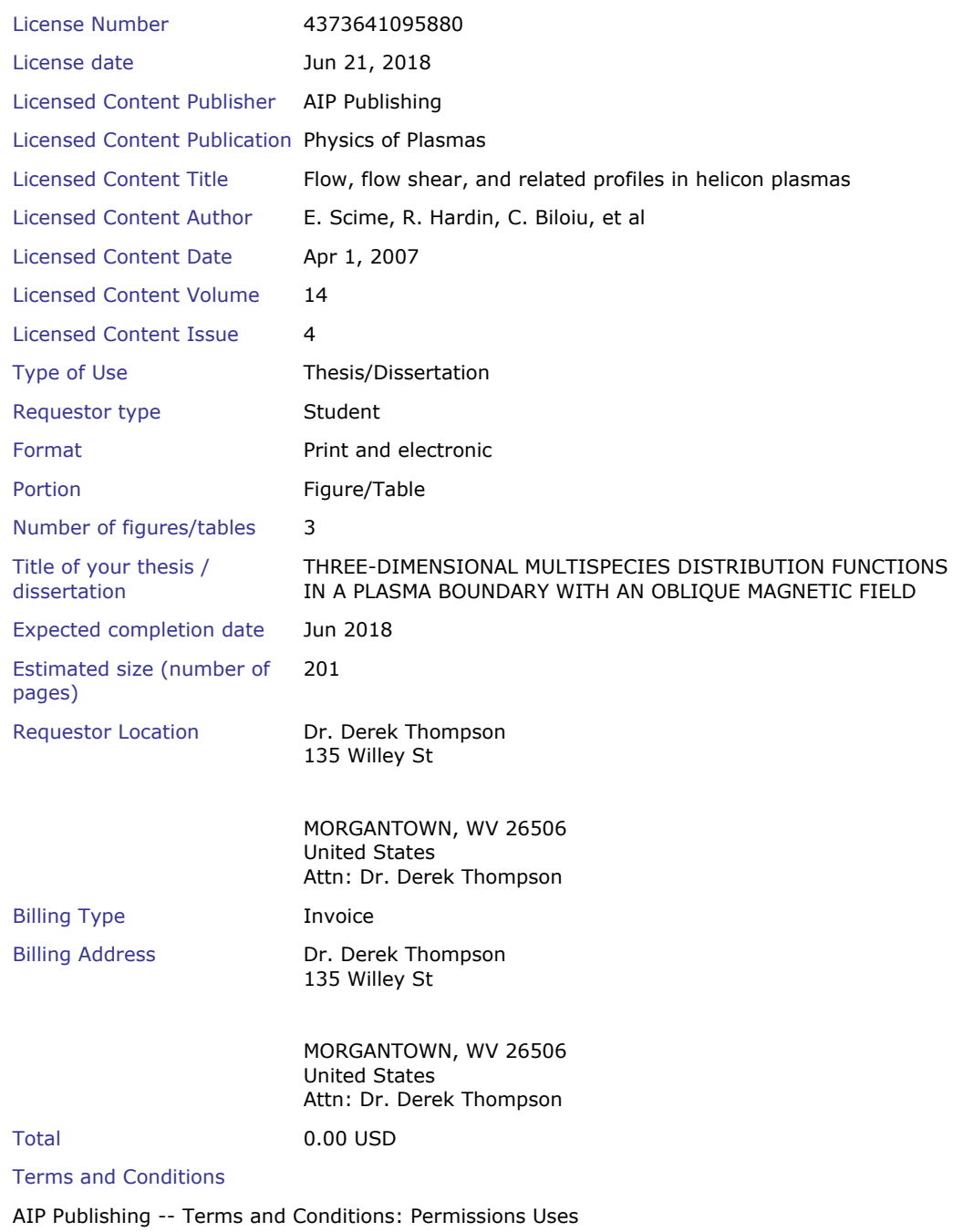

AIP Publishing -- Terms and Conditions: Permissions Uses 
This Agreement between Dr. Derek Thompson ("You") and AIP Publishing ("AIP

Publishing") consists of your license details and the terms and conditions provided by AIP Publishing and Copyright Clearance Center.

\begin{tabular}{|c|c|}
\hline License Number & 4372700079043 \\
\hline License date & Jun 19, 2018 \\
\hline Licensed Content Publisher & AIP Publishing \\
\hline Licensed Content Publication & Review of Scientific Instruments \\
\hline Licensed Content Title & $\begin{array}{l}\text { Laser induced fluorescence measurements of axial velocity, velocity } \\
\text { shear, and parallel ion temperature profiles during the route to } \\
\text { plasma turbulence in a linear magnetized plasma device }\end{array}$ \\
\hline Licensed Content Author & S. Chakraborty Thakur, K. Adriany, J. J. Gosselin, et al \\
\hline Licensed Content Date & Nov 1, 2016 \\
\hline Licensed Content Volume & 87 \\
\hline Licensed Content Issue & 11 \\
\hline Type of Use & Thesis/Dissertation \\
\hline Requestor type & Student \\
\hline Format & Print and electronic \\
\hline Portion & Figure/Table \\
\hline Number of figures/tables & 1 \\
\hline $\begin{array}{l}\text { Title of your thesis / } \\
\text { dissertation }\end{array}$ & $\begin{array}{l}\text { THREE-DIMENSIONAL MULTISPECIES DISTRIBUTION FUNCTIONS } \\
\text { IN A PLASMA BOUNDARY WITH AN OBLIQUE MAGNETIC FIELD }\end{array}$ \\
\hline Expected completion date & Jun 2018 \\
\hline $\begin{array}{l}\text { Estimated size (number of } \\
\text { pages) }\end{array}$ & 201 \\
\hline Requestor Location & $\begin{array}{l}\text { Dr. Derek Thompson } \\
135 \text { Willey St }\end{array}$ \\
\hline & $\begin{array}{l}\text { MORGANTOWN, WV } 26506 \\
\text { United States } \\
\text { Attn: Dr. Derek Thompson }\end{array}$ \\
\hline Billing Type & Invoice \\
\hline Billing Address & $\begin{array}{l}\text { Dr. Derek Thompson } \\
135 \text { Willey St }\end{array}$ \\
\hline & $\begin{array}{l}\text { MORGANTOWN, WV } 26506 \\
\text { United States } \\
\text { Attn: Dr. Derek Thompson }\end{array}$ \\
\hline Total & 0.00 USD \\
\hline
\end{tabular}


This Agreement between Dr. Derek Thompson ("You") and AIP Publishing ("AIP

Publishing") consists of your license details and the terms and conditions provided by AIP Publishing and Copyright Clearance Center.

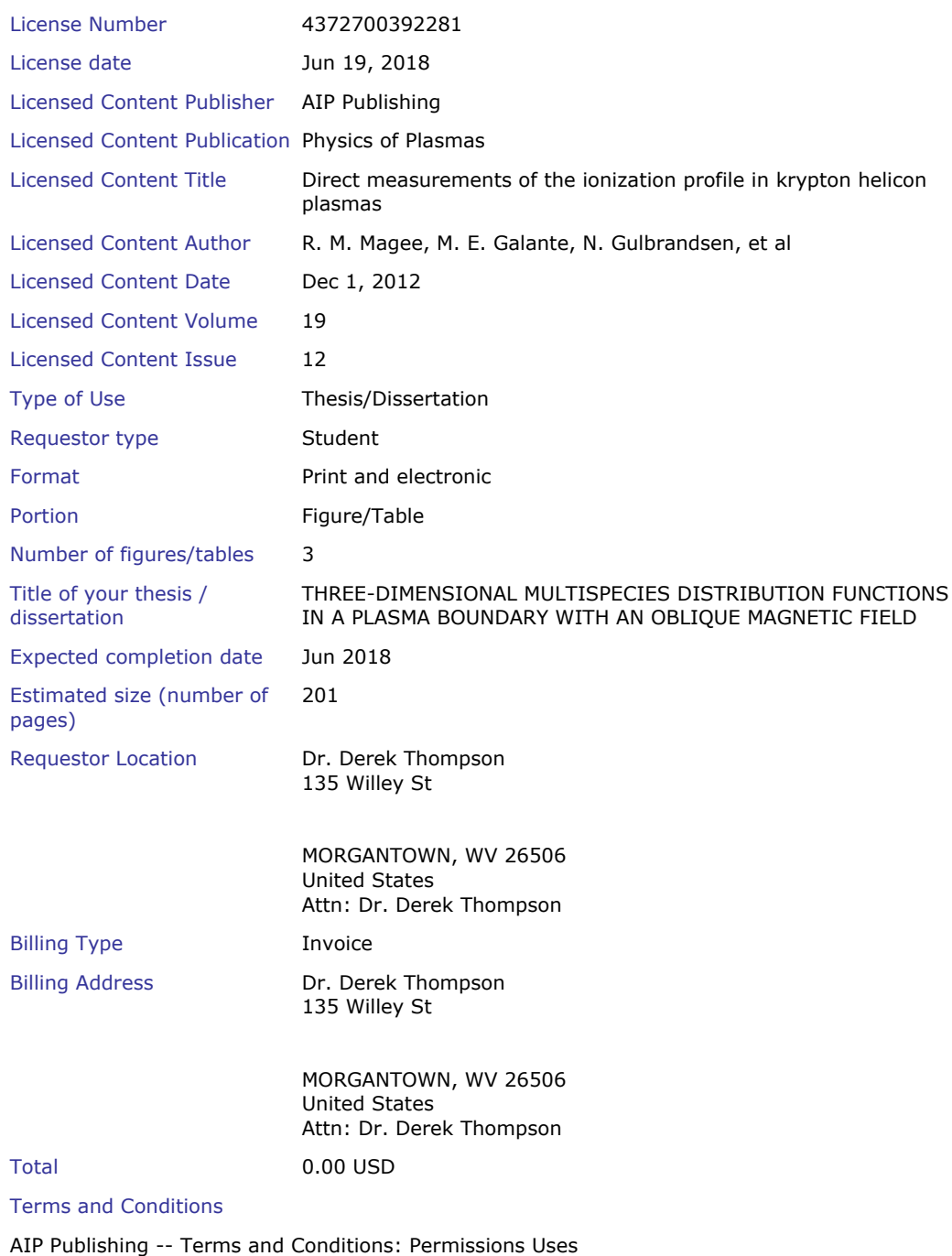


This Agreement between Dr. Derek Thompson ("You") and Cambridge University Press ("Cambridge University Press") consists of your license details and the terms and conditions provided by Cambridge University Press and Copyright Clearance Center.

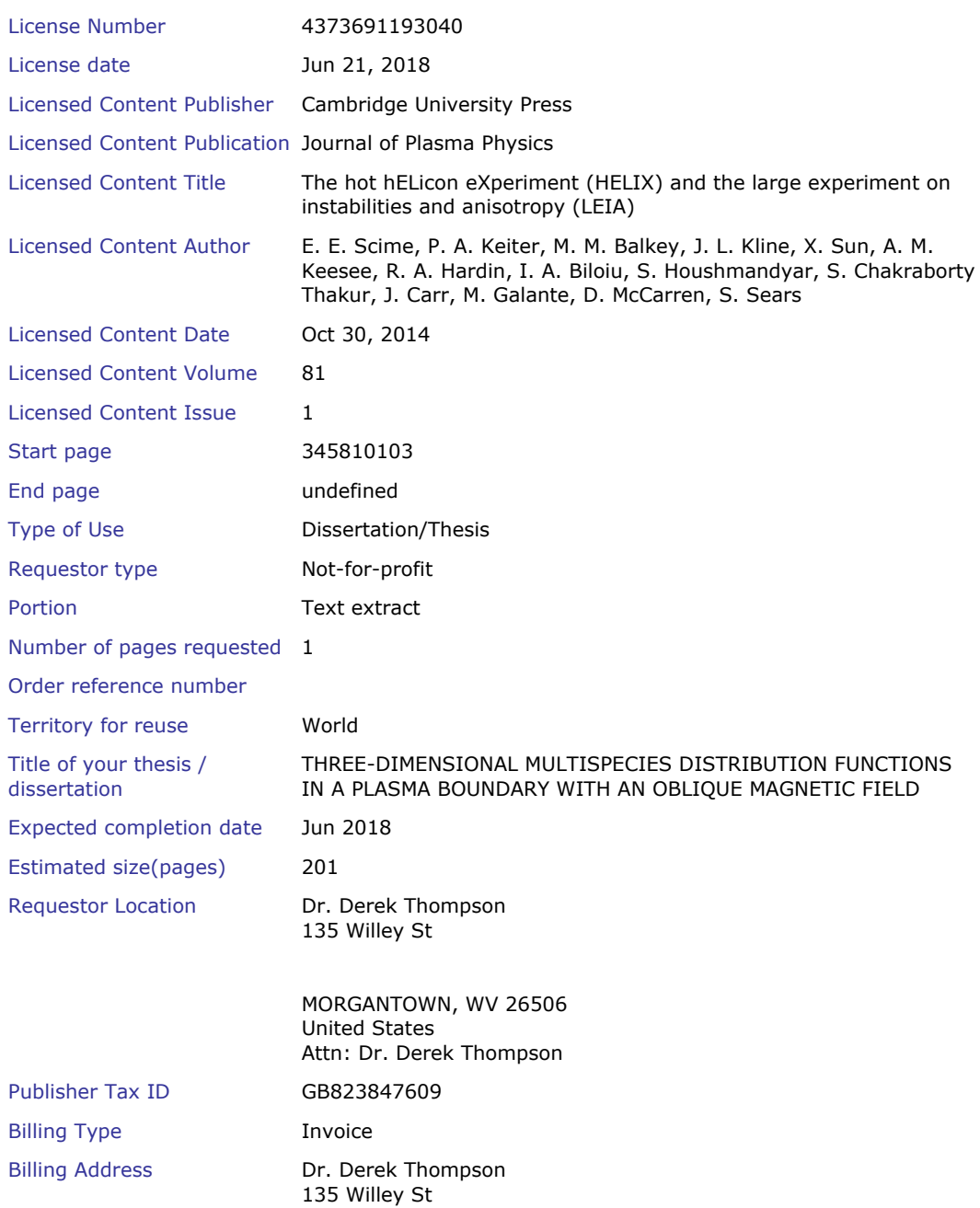


MORGANTOWN, WV 26506

United States

Attn: Dr. Derek Thompson

Total

0.00 USD

Terms and Conditions

\section{TERMS \& CONDITIONS}

Cambridge University Press grants the Licensee permission on a non-exclusive nontransferable basis to reproduce, make available or otherwise use the Licensed content 'Content' in the named territory 'Territory' for the purpose listed 'the Use' on Page 1 of this Agreement subject to the following terms and conditions.

1. The License is limited to the permission granted and the Content detailed herein and does not extend to any other permission or content.

2. Cambridge gives no warranty or indemnity in respect of any third-party copyright material included in the Content, for which the Licensee should seek separate permission clearance.

3. The integrity of the Content must be ensured.

4. The License does extend to any edition published specifically for the use of handicapped or reading-impaired individuals.

5. The Licensee shall provide a prominent acknowledgement in the following format: author/s, title of article, name of journal, volume number, issue number, page references, reproduced with permission.

Other terms and conditions:

v1.0

Questions? customercare@copyright.com or +1-855-239-3415 (toll free in the US) or +1-978-646-2777. 
This Agreement between Dr. Derek Thompson ("You") and AIP Publishing ("AIP Publishing") consists of your license details and the terms and conditions provided by AIP Publishing and Copyright Clearance Center.

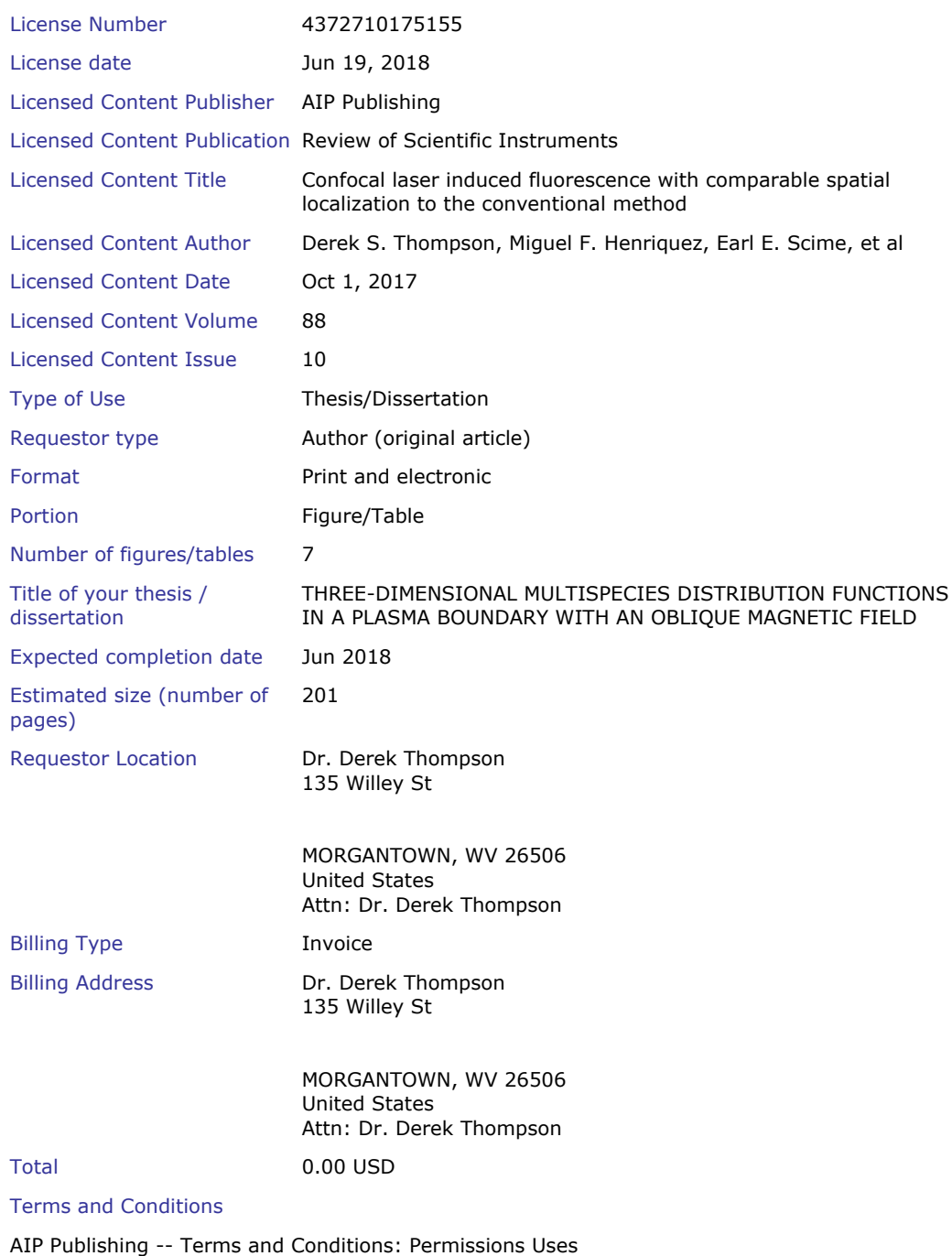




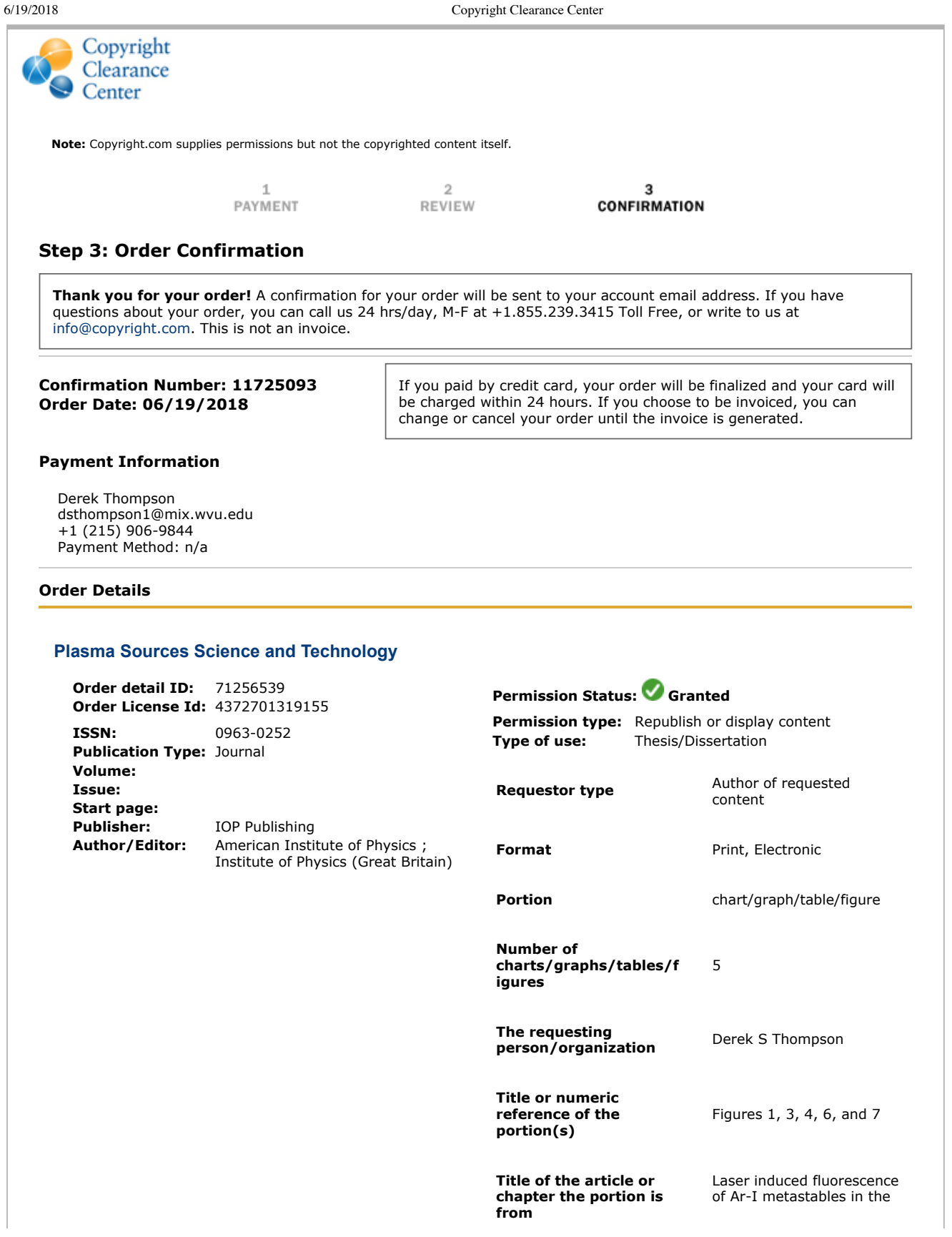




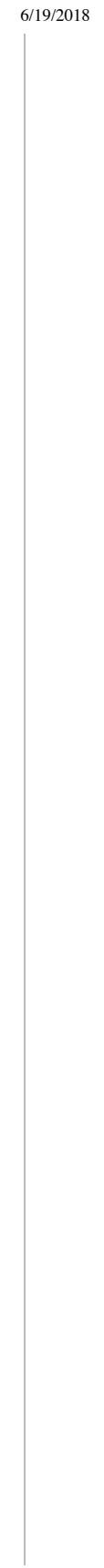

Copyright Clearance Center

presence of a magnetic

N/A

Author of portion(s)

Derek S Thompson

Volume of serial or monograph

N/A

Page range of portion

N/A

Publication date of portion

N/A

Rights for

Main product and any product related to main product

Duration of use

Life of current and all future editions

Creation of copies for the disabled

yes

With minor editing

privileges

no

For distribution to

Worldwide

In the following language(s)

Original language of publication

With incidental promotional use

Lifetime unit quantity of new product

Up to $1,000,000$

THREE-DIMENSIONAL

MULTISPECIES

Title

FUNCTIONS IN A PLASMA

BOUNDARY WITH AN

OBLIQUE MAGNETIC

FIELD

Instructor name

$n / a$

Institution name

$n / a$

Expected presentation

Jun 2018 
6/19/2018

Copyright Clearance Center

Confirmation Number: 11725093

Special Rightsholder Terms \& Conditions

The following terms \& conditions apply to the specific publication under which they are listed

Plasma Sources Science and Technology

Type of use: Thesis/Dissertation

\section{TERMS AND CONDITIONS}

The following terms are individual to this publisher:

These special terms and conditions are in addition to the standard terms and conditions for CCC's Republication Service and, together with those standard terms and conditions, govern the use of the Works.

As the "User" you will make all reasonable efforts to contact the author(s) of the article which the Work is to be reused from, to seek consent for your intended use. Contacting one author who is acting expressly as authorised agent for their co-author(s) is acceptable.

User will reproduce the following wording prominently alongside the Work:

- the source of the Work, including author, article title, title of journal, volume number, issue number (if relevant), page range (or first page if this is the only information available) and date of first publication. This information can be contained in a footnote or reference note; and

- a link back to the article (via DOI); and

- if practicable, and IN ALL CASES for new works published under any of the Creative Commons licences, the words "C IOP Publishing. Reproduced with permission. All rights reserved"

Without the express permission of the author(s) and the Rightsholder of the article from which the Work is to be reused, User shall not use it in any way which, in the opinion of the Rightsholder, could: (i) distort or alter the author(s)' original intention(s) and meaning; (ii) be prejudicial to the honour or reputation of the author(s); and/or (iii) imply endorsement by the author(s) and/or the Rightsholder.

This licence does not apply to any article which is credited to another source and which does not have the copyright line '(c) IOP Publishing Ltd'. User must check the copyright line of the article from which the Work is to be reused to check that IOP Publishing Ltd has all the necessary rights to be able to grant permission. User is solely responsible for identifying and obtaining separate licences and permissions from the copyright owner for reuse of any such third party material/figures which the Rightsholder is not the copyright owner of. The Rightsholder shall not reimburse any fees which User pays for a republication license for such third party content.

This licence does not apply to any material/figure which is credited to another source in the Rightsholder's publication or has been obtained from a third party. User must check the Version of Record of the article from which the Work is to be reused, to check whether any of the material in the Work is third party material. Third party citations and/or copyright notices and/or permissions statements may not be included in any other version of the article from which the Work is to be reused and so cannot be relied upon by the User. User is solely responsible for identifying and obtaining separate licences and permissions from the copyright owner for reuse of any such third party material/figures where the Rightsholder is not the copyright owner. The Rightsholder shall not reimburse any fees which User pays for republication license for such third party content.

User and CCC acknowledge that the Rightsholder may, from time to time, make changes or additions to these special terms and conditions without express notification, provided that these shall not apply to permissions already secured and paid for by User prior to such change or addition.

User acknowledges that the Rightsholder (which includes companies within its group and third parties for whom it publishes its titles) may make use of personal data collected through the service in the course of their business.

If User is the author of the Work, User may automatically have the right to reuse it under the rights granted back when User transferred the copyright in the article to the Rightsholder. User should check the copyright form and the relevant author rights policy to check whether permission is required. If User is the author of the Work and does require permission for proposed reuse of the Work, User should select 'Author of requested content' as the Requestor Type. The Rightsholder shall not reimburse any fees which User pays for a republication license.

If User is the author of the article which User wishes to reuse in User's thesis or dissertation, the republication licence covers the right to include the Accepted Manuscript version (not the Version of Record) of the article. User must include citation details and, for online use, a link to the Version of Record of the article on the Rightsholder's website. User may need to obtain separate permission for any third party content included within the article. User must check this with the copyright owner of such third party content. User may not include the article in a thesis or dissertation which is published by ProQuest. Any other commercial use of User's thesis or dissertation containing the article would also need to be 
expressly notified in writing to the Rightsholder at the time of request and would require separate written permission from the Rightsholder.

User does not need to request permission for Work which has been published under a CC BY licence. User must check the Version of Record of the CC BY article from which the Work is to be reused, to check whether any of the material in the Work is third party material and so not published under the CC BY licence. User is solely responsible for identifying and obtaining separate licences and permissions from the copyright owner for reuse of any such third party material/figures. The Rightsholder shall not reimburse any fees which User pays for such licences and permissions.

As well as CCC, the Rightsholder shall have the right to bring any legal action that it deems necessary to enforce its rights should it consider that the Work infringes those rights in any way.

For STM Signatories ONLY (as agreed as part of the STM Guidelines).

Any licence granted for a particular edition of a Work will apply also to subsequent editions of it and for editions in other languages, provided such editions are for the Work as a whole in situ and do not involve the separate exploitation of the permitted illustrations or excerpts.

\section{Other Terms and Conditions:}

\section{STANDARD TERMS AND CONDITIONS}

1. Description of Service; Defined Terms. This Republication License enables the User to obtain licenses for republication of one or more copyrighted works as described in detail on the relevant Order Confirmation (the "Work(s)"). Copyright Clearance Center, Inc. ("CCC") grants licenses through the Service on behalf of the rightsholder identified on the Order Confirmation (the "Rightsholder"). "Republication", as used herein, generally means the inclusion of a Work, in whole or in part, in a new work or works, also as described on the Order Confirmation. "User", as used herein, means the person
or entity making such republication.

2. The terms set forth in the relevant Order Confirmation, and any terms set by the Rightsholder with respect to a particular Work, govern the terms of use of Works in connection with the Service. By using the Service, the person transacting for a republication license on behalf of the User represents and warrants that he/she/it (a) has been duly authorized by the User to accept, and hereby does accept, all such terms and conditions on behalf of User, and (b) shall inform User of all such terms and conditions. In the event such person is a "freelancer" or other third party independent of User and CCC, such party shall be deemed jointly a "Usen" for purposes of these terns and conditions. In any event, User shall be deemed to have accepted and agreed to all such terms and conditions if User republishes the Work in any

\section{Scope of License; Limitations and Obligations.}

3.1 All Works and all rights therein, including copyright rights, remain the sole and exclusive property of the Rightsholder. The license created by the exchange of an Order Confirmation (and/or any invoice) and payment by User of the full amount set forth on that document includes only those rights expressly set forth in the Order Confirmation and in these terms and conditions, and conveys no other rights in the Work(s) to User. All rights not expressly granted are hereby reserved.

3.2 General Payment Terms: You may pay by credit card or through an account with us payable at the end of the month. If you and we agree that you may establish a standing account with CCC, then the following terms apply: Remit Payment to: Copyright Clearance Center, 29118 Network Place, Chicago, IL 60673-1291. Payments Due: Invoices are payable upon their delivery to you (or upon our notice to you that they are available to you for downloading). After 30 days, outstanding amounts will be subject to a service charge of $1-1 / 2 \%$ per month or, if less, the maximum rate allowed by applicable law. Unless otherwise specifically set forth in the Order Confirmation or in a separate written agreement signed by CCC, invoices are due and payable on "net 30 " terms. While User may exercise the rights licensed immediately upo issuance of the Order Confirmation, the license is automatically revoked and is null and void, as if it had never been issued, if complete payment for the license is not received on a timely basis either from User directly or through a
payment agent, such as a credit card company.

3.3 Unless otherwise provided in the Order Confirmation, any grant of rights to User (i) is "one-time" (including the editions and product family specified in the license), (ii) is non-exclusive and non-transferable and (iii) is subject to any and all limitations and restrictions (such as, but not limited to, limitations on duration of use or circulation) included in the Order Confirmation or invoice and/or in these terms and conditions. Upon completion of the licensed use, User shall either secure a new permission for further use of the Work(s) or immediately cease any new use of the Work(s) and shall render inaccessible (such as by deleting or by removing or severing links or other locators) any further copies of the Work

3.4 In the event that the material for which a republication license is sought includes third party materials (such as photographs, illustrations, graphs, inserts and similar materials) which are identified in such material as having been used by permission, User is responsible for identifying, and seeking separate licenses (under this Service or otherwise) for, any of such third party materials; without a separate license, such third party materials may not be used.

3.5 Use of proper copyright notice for a Work is required as a condition of any license granted under the Service. Unless otherwise provided in the Order Confirmation, a proper copyright notice will read substantially as follows: "Republished with permission of [Rightsholder's name], from [Work's title, author, volume, edition number and year of copyright]; permission conveyed through Copyright Clearance Center, Inc. " Such notice must be provided in a reasonably legible font size and must be placed either immediately adjacent to the Work as used (for example, as part of a by-line or 
footnote but not as a separate electronic link) or in the place where substantially all other credits or notices for the new work containing the republished Work are located. Failure to include the required notice results in loss to the Rightsholder and CCC, and the User shall be liable to pay liquidated damages for each such failure equal to twice the use fee specified in the Order Confirmation, in addition to the use fee itself and any other fees and charges specified.

3.6 User may only make alterations to the Work if and as expressly set forth in the Order Confirmation. No Work may be used in any way that is defamatory, violates the rights of third parties (including such third parties' rights of copyright, privacy, publicity, or other tangible or intangible property), or is otherwise illegal, sexually explicit or obscene. In addition, User may not conjoin a Work with any other material that may result in damage to the reputation of the cooperate with any reasonable resuect of $C C C$ or the Rightsholder in connection therewith.

4. Indemnity. User hereby indemnifies and agrees to defend the Rightsholder and CCC, and their respective employees and directors, against all claims, liability, damages, costs and expenses, including legal fees and expenses, arising out of unauthorized way by User, including claims of defamation or infringement of rights of copyright, publicity, privacy or other tangible or intangible property.

5. Limitation of Liability. UNDER NO CIRCUMSTANCES WILL CCC OR THE RIGHTSHOLDER BE LIABLE FOR ANY DIRECT, INDIRECT, CONSEQUENTIAL OR INCIDENTAL DAMAGES (INCLUDING WITHOUT LIMITATION DAMAGES FOR LOSS OF BUSINESS PROFITS OR INFORMATION, OR FOR BUSINESS INTERRUPTION) ARISING OUT OF THE USE OR INABILITY TO USE A WORK, EVEN IF ONE OF THEM HAS BEEN ADVISED OF THE POSSIBILITY OF SUCH DAMAGES. In any event, the total liability of the Rightsholder and CCC (including their respective employees and directors) shall not exceed the total amount actually paid by User for this license. User assumes full liability for the actions and omissions of its principals, employees, agents, affiliates, successors and assigns.

6. Limited Warranties. THE WORK(S) AND RIGHT(S) ARE PROVIDED "AS IS". CCC HAS THE RIGHT TO GRANT TO USER THE RIGHTS GRANTED IN THE ORDER CONFIRMATION DOCUMENT. CCC AND THE RIGHTSHOLDER DISCLAIM ALL OTHER WARRANTIES RELATING TO THE WORK(S) AND RIGHT(S), EITHER EXPRESS OR IMPLIED, INCLUDING WITHOUT LIMITATION IMPLIED WARRANTIES OF MERCHANTABILITY OR FITNESS FOR A PARTICULAR PURPOSE. ADDITIONA RIGHTS MAY BE REQUIRED TO USE ILLUSTRATIONS, GRAPHS, PHOTOGRAPHS, ABSTRACTS, INSERTS OR OTHER
PORTIONS OF THE WORK (AS OPPOSED TO THE ENTIRE WORK) IN A MANNER CONTEMPLATED BY USER; USER UNDERSTANDS AND AGREES THAT NEITHER CCC NOR THE RIGHTSHOLDER MAY HAVE SUCH ADDITIONAL RIGHTS TO GRANT.

7. Effect of Breach. Any failure by User to pay any amount when due, or any use by User of a Work beyond the scope of the license set forth in the Order Confirmation and/or these terms and conditions, shall be a material breach of the license created by the Order Confirmation and these terms and conditions. Any breach not cured within 30 days of written notice thereof shall result in immediate termination of such license without further notice. Any unauthorized (but licensable) use of a Work that is terminated immediately upon ne without further notice. Any unauthorized (but licensable) use of a Work that is terminated immediately upon notice thereof may be liquidated by payment of the
Rightsholder's ordinary license price therefor; any unauthorized (and unlicensable) use that is not terminated
immediately for any reason (including, for example, because materials containing the Work cannot reasonably be immediately for any reason (including, for example, because materials containing the Work cannot reasonably be
recalled) will be subject to all remedies available at law or in equity, but in no event to a payment of less than three recalled) will be subject to all remedies available at law or in equity, but in no event to a payment of less than three CCC's costs and expenses incurred in collecting such payment.

\section{Miscellaneous.}

8.1 User acknowledges that CCC may, from time to time, make changes or additions to the Service or to these terms and conditions, and CCC reserves the right to send notice to the User by electronic mail or otherwise for the purposes of notifying User of such changes or additions; provided that any such changes or additions shall not apply to permissions already secured and paid for.

8.2 Use of User-related information collected through the Service is governed by CCC's privacy policy, available online here: http://www.copyright.com/content/cc3/en/tools/footer/privacypolicy.html.

8.3 The licensing transaction described in the Order Confirmation is personal to User. Therefore, User may not assign or transfer to any other person (whether a natural person or an organization of any kind) the license created by the Order Confirmation and these terms and conditions or any rights granted hereunder; provided, however, that User may assign such license in its entirety on written notice to CCC in the event of a transfer of all or substantially all of User's rights in the new material which includes the Work(s) licensed under this Service.

8.4 No amendment or waiver of any terms is binding unless set forth in writing and signed by the parties. The Rightsholder and CCC hereby object to any terms contained in any writing prepared by the User or its principals, employees, agents or affiliates and purporting to govern or otherwise relate to the licensing transaction described in the Order Confirmation, which terms are in any way inconsistent with any terms set forth in the Order Confirmation and/or in these terms and conditions or CCC's standard operating procedures, whether such writing is prepared prior to, simultaneously with or subsequent to the Order Confirmation, and whether such writing appears on a copy of the Order Confirmation or in a separate instrument.

8.5 The licensing transaction described in the Order Confirmation document shall be governed by and construed under the law of the State of New York, USA, without regard to the principles thereof of conflicts of law. Any case, controversy, suit, action, or proceeding arising out of, in connection with, or related to such licensing transaction shall be brought, at CCC's sole discretion, in any federal or state court located in the County of New York, State of New York, USA, or in an federal or state court whose geographical jurisdiction covers the location of the Rightsholder set forth in the Orde Confirmation. The parties expressly submit to the personal jurisdiction and venue of each such federal or state court.If 
$6 / 20 / 2018$

This Agreement between Dr. Derek Thompson ("You") and Elsevier ("Elsevier") consists of your license details and the terms and conditions provided by Elsevier and Copyright Clearance Center.

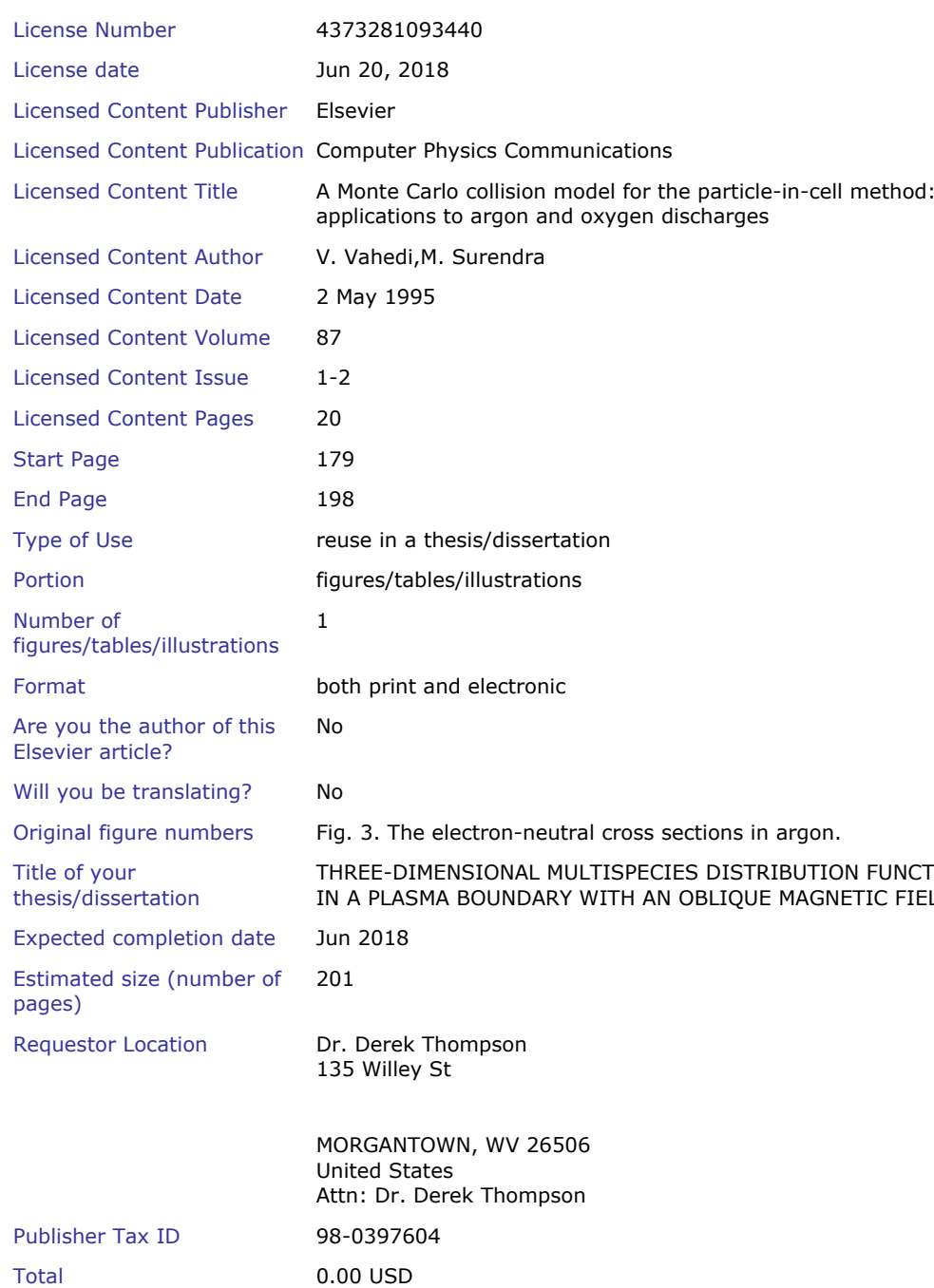


6/20/2018

RightsLink Printable License

Terms and Conditions

\section{INTRODUCTION}

1. The publisher for this copyrighted material is Elsevier. By clicking "accept" in connection with completing this licensing transaction, you agree that the following terms and conditions apply to this transaction (along with the Billing and Payment terms and conditions established by Copyright Clearance Center, Inc. ("CCC"), at the time that you opened your Rightslink account and that are available at any time at http://myaccount.copyright.com).

\section{GENERAL TERMS}

2. Elsevier hereby grants you permission to reproduce the aforementioned material subject to the terms and conditions indicated.

3. Acknowledgement: If any part of the material to be used (for example, figures) has appeared in our publication with credit or acknowledgement to another source, permission must also be sought from that source. If such permission is not obtained then that material may not be included in your publication/copies. Suitable acknowledgement to the source must be made, either as a footnote or in a reference list at the end of your publication, as follows:

"Reprinted from Publication title, Vol /edition number, Author(s), Title of article / title of chapter, Pages No., Copyright (Year), with permission from Elsevier [OR APPLICABLE

SOCIETY COPYRIGHT OWNER]." Also Lancet special credit - "Reprinted from The

Lancet, Vol. number, Author(s), Title of article, Pages No., Copyright (Year), with permission from Elsevier."

4. Reproduction of this material is confined to the purpose and/or media for which permission is hereby given.

5. Altering/Modifying Material: Not Permitted. However figures and illustrations may be altered/adapted minimally to serve your work. Any other abbreviations, additions, deletions and/or any other alterations shall be made only with prior written authorization of Elsevier Ltd. (Please contact Elsevier at permissions@elsevier.com). No modifications can be made to any Lancet figures/tables and they must be reproduced in full.

6. If the permission fee for the requested use of our material is waived in this instance, please be advised that your future requests for Elsevier materials may attract a fee. 7. Reservation of Rights: Publisher reserves all rights not specifically granted in the combination of (i) the license details provided by you and accepted in the course of this licensing transaction, (ii) these terms and conditions and (iii) CCC's Billing and Payment terms and conditions.

8. License Contingent Upon Payment: While you may exercise the rights licensed immediately upon issuance of the license at the end of the licensing process for the transaction, provided that you have disclosed complete and accurate details of your proposed use, no license is finally effective unless and until full payment is received from you (either by publisher or by $\mathrm{CCC}$ ) as provided in CCC's Billing and Payment terms and conditions. If full payment is not received on a timely basis, then any license preliminarily granted shall be deemed automatically revoked and shall be void as if never granted. Further, in the event that you breach any of these terms and conditions or any of CCC's Billing and Payment terms and conditions, the license is automatically revoked and shall be void as if never granted. Use of materials as described in a revoked license, as well as any use of the materials beyond the scope of an unrevoked license, may constitute copyright infringement and publisher reserves the right to take any and all action to protect its copyright in the materials.

9. Warranties: Publisher makes no representations or warranties with respect to the licensed material.

10. Indemnity: You hereby indemnify and agree to hold harmless publisher and CCC, and their respective officers, directors, employees and agents, from and against any and all 
claims arising out of your use of the licensed material other than as specifically authorized pursuant to this license.

11. No Transfer of License: This license is personal to you and may not be sublicensed, assigned, or transferred by you to any other person without publisher's written permission.

12. No Amendment Except in Writing: This license may not be amended except in a writing signed by both parties (or, in the case of publisher, by CCC on publisher's behalf).

13. Objection to Contrary Terms: Publisher hereby objects to any terms contained in any purchase order, acknowledgment, check endorsement or other writing prepared by you, which terms are inconsistent with these terms and conditions or CCC's Billing and Payment terms and conditions. These terms and conditions, together with CCC's Billing and Payment terms and conditions (which are incorporated herein), comprise the entire agreement between you and publisher (and CCC) concerning this licensing transaction. In the event of any conflict between your obligations established by these terms and conditions and those established by CCC's Billing and Payment terms and conditions, these terms and conditions shall control.

14. Revocation: Elsevier or Copyright Clearance Center may deny the permissions described in this License at their sole discretion, for any reason or no reason, with a full refund payable to you. Notice of such denial will be made using the contact information provided by you. Failure to receive such notice will not alter or invalidate the denial. In no event will Elsevier or Copyright Clearance Center be responsible or liable for any costs, expenses or damage incurred by you as a result of a denial of your permission request, other than a refund of the amount(s) paid by you to Elsevier and/or Copyright Clearance Center for denied permissions.

\section{LIMITED LICENSE}

The following terms and conditions apply only to specific license types: 15. Translation: This permission is granted for non-exclusive world English rights only unless your license was granted for translation rights. If you licensed translation rights you may only translate this content into the languages you requested. A professional translator must perform all translations and reproduce the content word for word preserving the integrity of the article.

16. Posting licensed content on any Website: The following terms and conditions apply as follows: Licensing material from an Elsevier journal: All content posted to the web site must maintain the copyright information line on the bottom of each image; A hyper-text must be included to the Homepage of the journal from which you are licensing at http://www.sciencedirect.com/science/journal/xxxxx or the Elsevier homepage for books at http://www.elsevier.com; Central Storage: This license does not include permission for a scanned version of the material to be stored in a central repository such as that provided by Heron/XanEdu.

Licensing material from an Elsevier book: A hyper-text link must be included to the Elsevier homepage at http://www.elsevier.com . All content posted to the web site must maintain the copyright information line on the bottom of each image.

Posting licensed content on Electronic reserve: In addition to the above the following clauses are applicable: The web site must be password-protected and made available only to bona fide students registered on a relevant course. This permission is granted for 1 year only. You may obtain a new license for future website posting.

17. For journal authors: the following clauses are applicable in addition to the above: Preprints:

A preprint is an author's own write-up of research results and analysis, it has not been peerreviewed, nor has it had any other value added to it by a publisher (such as formatting, copyright, technical enhancement etc.). 
Authors can share their preprints anywhere at any time. Preprints should not be added to or enhanced in any way in order to appear more like, or to substitute for, the final versions of articles however authors can update their preprints on arXiv or RePEc with their Accepted Author Manuscript (see below).

If accepted for publication, we encourage authors to link from the preprint to their formal publication via its DOI. Millions of researchers have access to the formal publications on ScienceDirect, and so links will help users to find, access, cite and use the best available version. Please note that Cell Press, The Lancet and some society-owned have different preprint policies. Information on these policies is available on the journal homepage. Accepted Author Manuscripts: An accepted author manuscript is the manuscript of an article that has been accepted for publication and which typically includes authorincorporated changes suggested during submission, peer review and editor-author communications

Authors can share their accepted author manuscript:

- immediately

- via their non-commercial person homepage or blog

- by updating a preprint in arXiv or RePEc with the accepted manuscript

- via their research institute or institutional repository for internal institutional uses or as part of an invitation-only research collaboration work-group

- directly by providing copies to their students or to research collaborators for their personal use

- for private scholarly sharing as part of an invitation-only work group on commercial sites with which Elsevier has an agreement

- After the embargo period

- via non-commercial hosting platforms such as their institutional repository

- via commercial sites with which Elsevier has an agreement

In all cases accepted manuscripts should:

- link to the formal publication via its DOI

- bear a CC-BY-NC-ND license - this is easy to do

- if aggregated with other manuscripts, for example in a repository or other site, be shared in alignment with our hosting policy not be added to or enhanced in any way to appear more like, or to substitute for, the published journal article.

Published journal article (JPA): A published journal article (PJA) is the definitive final record of published research that appears or will appear in the journal and embodies all value-adding publishing activities including peer review co-ordination, copy-editing, formatting, (if relevant) pagination and online enrichment.

Policies for sharing publishing journal articles differ for subscription and gold open access articles:

Subscription Articles: If you are an author, please share a link to your article rather than the full-text. Millions of researchers have access to the formal publications on ScienceDirect, and so links will help your users to find, access, cite, and use the best available version. Theses and dissertations which contain embedded PJAs as part of the formal submission can be posted publicly by the awarding institution with DOI links back to the formal publications on ScienceDirect.

If you are affiliated with a library that subscribes to ScienceDirect you have additional private sharing rights for others' research accessed under that agreement. This includes use for classroom teaching and internal training at the institution (including use in course packs and courseware programs), and inclusion of the article for grant funding purposes. 


\author{
6/20/2018_RightsLink Printable License
}

Gold Open Access Articles: May be shared according to the author-selected end-user license and should contain a CrossMark logo, the end user license, and a DOI link to the formal publication on ScienceDirect.

Please refer to Elsevier's posting policy for further information

18. For book authors the following clauses are applicable in addition to the above: Authors are permitted to place a brief summary of their work online only. You are not allowed to download and post the published electronic version of your chapter, nor may you scan the printed edition to create an electronic version. Posting to a repository: Authors are permitted to post a summary of their chapter only in their institution's repository.

19. Thesis/Dissertation: If your license is for use in a thesis/dissertation your thesis may be submitted to your institution in either print or electronic form. Should your thesis be published commercially, please reapply for permission. These requirements include permission for the Library and Archives of Canada to supply single copies, on demand, of the complete thesis and include permission for Proquest/UMI to supply single copies, on demand, of the complete thesis. Should your thesis be published commercially, please reapply for permission. Theses and dissertations which contain embedded PJAs as part of the formal submission can be posted publicly by the awarding institution with DOI links back to the formal publications on ScienceDirect.

\title{
Elsevier Open Access Terms and Conditions
}

You can publish open access with Elsevier in hundreds of open access journals or in nearly 2000 established subscription journals that support open access publishing. Permitted third party re-use of these open access articles is defined by the author's choice of Creative Commons user license. See our open access license policy for more information.

Terms \& Conditions applicable to all Open Access articles published with Elsevier: Any reuse of the article must not represent the author as endorsing the adaptation of the article nor should the article be modified in such a way as to damage the author's honour or reputation. If any changes have been made, such changes must be clearly indicated. The author(s) must be appropriately credited and we ask that you include the end user license and a DOI link to the formal publication on ScienceDirect.

If any part of the material to be used (for example, figures) has appeared in our publication with credit or acknowledgement to another source it is the responsibility of the user to ensure their reuse complies with the terms and conditions determined by the rights holder. Additional Terms \& Conditions applicable to each Creative Commons user license: CC BY: The CC-BY license allows users to copy, to create extracts, abstracts and new works from the Article, to alter and revise the Article and to make commercial use of the Article (including reuse and/or resale of the Article by commercial entities), provided the user gives appropriate credit (with a link to the formal publication through the relevant DOI), provides a link to the license, indicates if changes were made and the licensor is not represented as endorsing the use made of the work. The full details of the license are available at http://creativecommons.org/licenses/by/4.0.

CC BY NC SA: The CC BY-NC-SA license allows users to copy, to create extracts, abstracts and new works from the Article, to alter and revise the Article, provided this is not done for commercial purposes, and that the user gives appropriate credit (with a link to the formal publication through the relevant DOI), provides a link to the license, indicates if changes were made and the licensor is not represented as endorsing the use made of the work. Further, any new works must be made available on the same conditions. The full details of the license are available at http://creativecommons.org/licenses/by-nc-sa/4.0. CC BY NC ND: The CC BY-NC-ND license allows users to copy and distribute the Article, provided this is not done for commercial purposes and further does not permit distribution of the Article if it is changed or edited in any way, and provided the user gives appropriate credit (with a link to the formal publication through the relevant DOI), provides a link to the 
6/20/2018

RightsLink Printable License

license, and that the licensor is not represented as endorsing the use made of the work. The full details of the license are available at http://creativecommons.org/licenses/by-nc-nd/4.0 Any commercial reuse of Open Access articles published with a CC BY NC SA or CC BY NC ND license requires permission from Elsevier and will be subject to a fee.

Commercial reuse includes:

- Associating advertising with the full text of the Article

- Charging fees for document delivery or access

- Article aggregation

- Systematic distribution via e-mail lists or share buttons

Posting or linking by commercial companies for use by customers of those companies.

20. Other Conditions:

v1.9

Questions? customercare@copyright.com or +1-855-239-3415 (toll free in the US) or +1-978-646-2777. 
This Agreement between Dr. Derek Thompson ("You") and AIP Publishing ("AIP

Publishing") consists of your license details and the terms and conditions provided by AIP Publishing and Copyright Clearance Center.

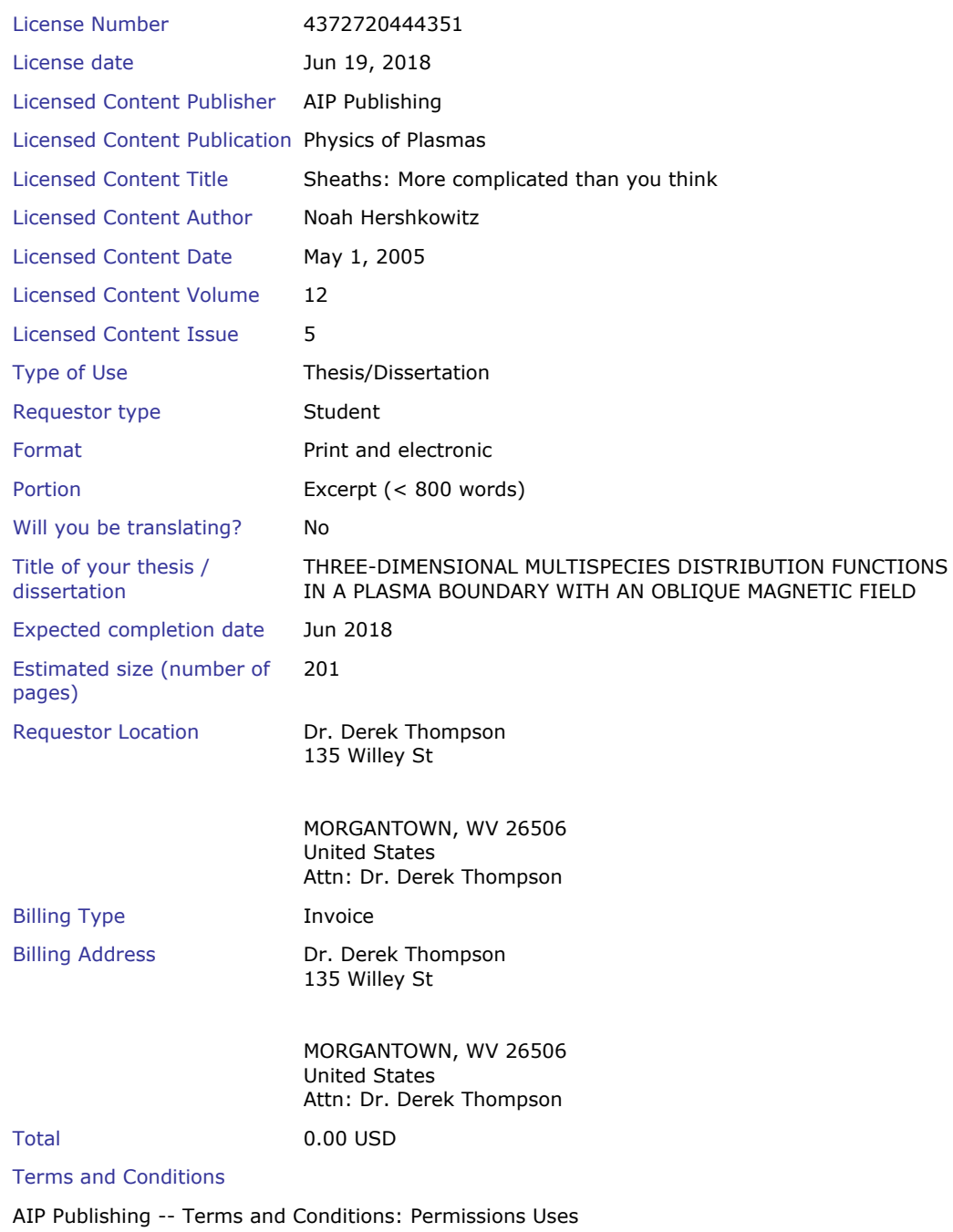

AIP Publishing -- Terms and Conditions: Permissions Uses 


$\begin{array}{ll}\text { Title: } & \text { Helicons-the early years } \\ \text { Author: } & \text { R.W. Boswell } \\ \text { Publication: } & \text { Plasma Science, IEEE } \\ & \text { Transactions on } \\ \text { Publisher: } & \text { IEEE } \\ \text { Date: } & \text { Dec. } 1997\end{array}$

Copyright ( 1997, IEEE

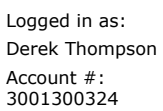

Thesis / Dissertation Reuse

The IEEE does not require individuals working on a thesis to obtain a formal reuse license, however, you may print out this statement to be used as a permission grant:

Requirements to be followed when using any portion (e.g., figure, graph, table, or textual material) of an IEEE copyrighted paper in a thesis:

1) In the case of textual material (e.g., using short quotes or referring to the work within these papers) users must give full credit to the original source (author, paper, publication) followed by the IEEE copyright line () 2011 IEEE.

2) In the case of illustrations or tabular material, we require that the copyright line (c) [Year of original publication] IEEE appear prominently with each reprinted figure and/or table.

3) If a substantial portion of the original paper is to be used, and if you are not the senior author, also obtain the senior author's approval.

Requirements to be followed when using an entire IEEE copyrighted paper in a thesis:

1) The following IEEE copyright/ credit notice should be placed prominently in the references: (c) [year of original publication] IEEE. Reprinted, with permission, from [author names, paper title, IEEE publication title, and month/year of publication]

2) Only the accepted version of an IEEE copyrighted paper can be used when posting the paper or your thesis on-line.

3 ) In placing the thesis on the author's university website, please display the following message in a prominent place on the website: In reference to IEEE copyrighted material which is used with permission in this thesis, the IEEE does not endorse any of [university/educational entity's name goes here]'s products or services. Internal or personal use of this material is permitted. If interested in reprinting/republishing IEEE copyrighted material for advertising or promotional purposes or for creating new collective works for resale or redistribution, please go to http://www.ieee.org/publications_standards/publications/rights/rights_link.html to learn how to obtain a License from RightsLink.

If applicable, University Microfilms and/or ProQuest Library, or the Archives of Canada may supply single copies of the dissertation. 
This Agreement between Dr. Derek Thompson ("You") and AIP Publishing ("AIP

Publishing") consists of your license details and the terms and conditions provided by AIP Publishing and Copyright Clearance Center.

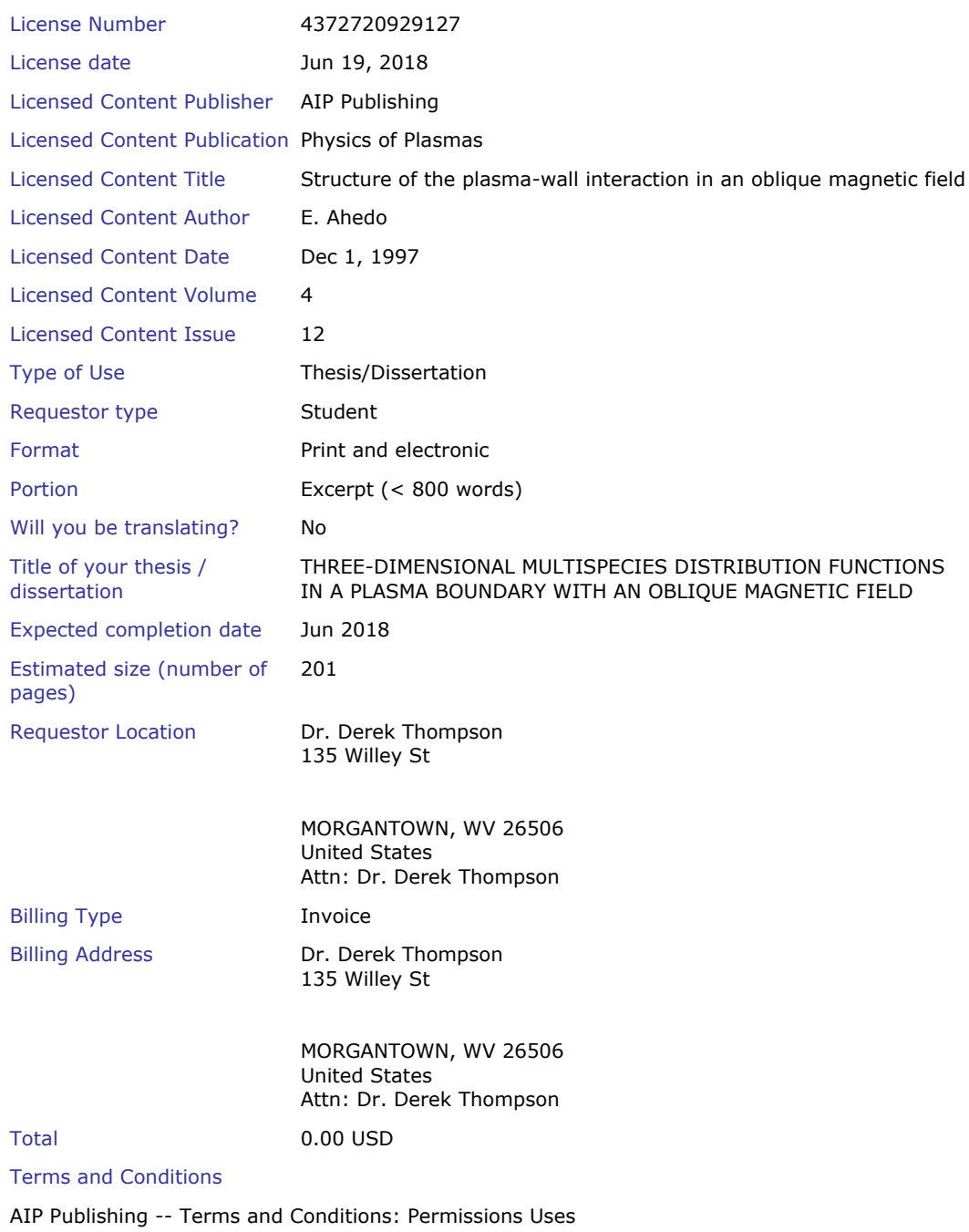

AIP Publishing -- Terms and Conditions: Permissions Uses 
From: Umair Siddiqui

Subject: Re: Figure permission request

Date: June 20, 2018 at 12:14 AM

To: Derek Thompson

Yes, I approve.

On Tue, Jun 19, 2018 at 9:00 PM Derek Thompson <

$>$ wrote:

Dr. M. Umair Siddiqui:

I am requesting permission to reproduce Figure 4.2 from your dissertation in my dissertation, which will be published in West Virginia University's library system and on ProQuest indefinitely. I will include all appropriate credit.

Thank you.

Sincerely,

Derek Thompson

Derek S. Thompson, Ph.D.

Postdoctoral research associate

Department of Physics \& Astronomy

West Virginia University

Linkedln

From: Rinat Khaziev

Subject: Re: Figure permission request

Date: June 19, 2018 at 8:49 PM

To: Derek Thompson

Dear Dr. Thompson,

I grant you permission to reproduce Figure 5.6 and 5.7 from my dissertation in your dissertation.

Best,

Rinat Khaziev, PhD

On Tue, 2018-06-19 at 15:05 -0400, Derek Thompson wrote

I am requesting permission to reproduce Figure 5.6 and 5.7 from your

dissertation in my dissertation, which will be published in West

Virginia University's library system and on ProQuest indefinitely. I

will include all appropriate credit. 


\section{Bibliography}

[1] A V Phelps. Cross sections and swarm coefficients for nitrogen ions and neutrals in $\mathrm{N}_{2}$ and argon ions and neutrals in ar for energies from $0.1 \mathrm{ev}$ to 10 kev. Journal of Physical and Chemical Reference Data, 20(3):557-573, 1991.

[2] Irving Langmuir. The interaction of electron and positive ion space charges in cathode sheaths. Physical Review, 33(6):954, 1929.

[3] Noah Hershkowitz. Sheaths: more complicated than you think. Physics of plasmas, 12(5):055502, 2005. URL https://aip.scitation.org/doi/abs/ 10.1063/1.1887189.

[4] Grzegorz Maslowski and Vladimir A Rakov. A study of the lightning channel corona sheath. Journal of Geophysical Research: atmospheres, 111(D14), 2006 .

[5] R. E. Ergun, L. Andersson, J. Tao, V. Angelopoulos, J. Bonnell, J. P. McFadden, D. E. Larson, S. Eriksson, T. Johansson, C. M. Cully, D. N. Newman, M. V. Goldman, A. Roux, O. LeContel, K.-H. Glassmeier, and W. Baumjohann. Observations of double layers in Earth's plasma sheet. Phys. Rev. Lett., 102:155002, Apr 2009. doi: 10.1103/PhysRevLett.102.155002. URL https://link.aps.org/doi/10.1103/PhysRevLett.102.155002.

[6] David Bohm. Minimum ionic kinetic theory for a stable sheath. In Andrew Guthrie and Raymond Kornelious Wakerling, editors, The characteristics of electrical discharge in magnetic fields, volume 5, pages 77-86. McGraw-Hill, New York, 1949.

[7] Greg Severn, Chi-Shung Yip, Noah Hershkowitz, and Scott D Baalrud. Experimental studies of ion flow near the sheath edge in multiple ion species plasma including argon, xenon and neon. Plasma Sources Science and Technology, 26(5):055021, 2017. URL http://stacks.iop.org/0963-0252/26/ $i=5 / a=055021$.

[8] R. Hood, B. Scheiner, S. D. Baalrud, M. M. Hopkins, E. V. Barnat, B. T. Yee, R. L. Merlino, and F. Skiff. Ion flow and sheath structure near positively biased electrodes. Physics of Plasmas, 23(11):113503, 2016. doi: 10.1063/1. 4967870. URL https://doi.org/10.1063/1.4967870. 
[9] Tilmann Lunt, Gerd Fussmann, and Ole Waldmann. Experimental investigation of the plasma-wall transition. Phys. Rev. Lett., 100:175004, May 2008. doi: 10.1103/PhysRevLett.100.175004. URL https://link.aps.org/doi/ 10.1103/PhysRevLett.100.175004.

[10] R. Chodura. Plasma-wall transition in an oblique magnetic field. The Physics of Fluids, 25(9):1628-1633, 1982. doi: 10.1063/1.863955. URL https: //aip. scitation.org/doi/abs/10.1063/1.863955.

[11] E Ahedo. Structure of the plasma-wall interaction in an oblique magnetic field. Physics of Plasmas, 4(12):4419-4430, 1997. URL https: //aip.scitation.org/doi/abs/10.1063/1.872606.

[12] Th Daube, K-U Riemann, and H Schmitz. Particle simulation of a magnetized plasma contacting the wall. Physics of Plasmas, 5(1):117-126, 1998.

[13] D Tskhakaya and S Kuhn. The magnetised plasma-wall transition: theory and PIC simulation. Contributions to Plasma Physics, 44(5-6):564-570, 2004.

[14] Stéphane Devaux and Giovanni Manfredi. Magnetized plasma-wall transition: consequences for wall sputtering and erosion. Plasma Physics and Controlled Fusion, 50(2):025009, 2008.

[15] K-U Riemann. The Bohm criterion and sheath formation. Journal of Physics D: Applied Physics, 24(4):493, 1991.

[16] R. Khaziev and D. Curreli. Ion energy-angle distribution functions at the plasma-material interface in oblique magnetic fields. Physics of Plasmas, 22 (4), 2015. ISSN 10897674 1070664X. doi: 10.1063/1.4916910.

[17] Alessandro Geraldini, Felix I Parra, and Fulvio Militello. Gyrokinetic treatment of a grazing angle magnetic presheath. Plasma Physics and Controlled Fusion, 59(2):025015, 2017.

[18] JA Meyer, G-H Kim, MJ Goeckner, and N Hershkowitz. Measurements of the presheath in an electron cyclotron resonance etching device. Plasma Sources Science and Technology, 1(3):147, 1992.

[19] G-H Kim, N Hershkowitz, DA Diebold, and M-H Cho. Magnetic and collisional effects on presheaths. Physics of Plasmas, 2(8):3222-3233, 1995.

[20] Bornali Singha, A Sarma, and Joyanti Chutia. Experimental observation of sheath and magnetic presheath over an oblique metallic plate in the presence of a magnetic field. Physics of Plasmas, 9(2):683-690, 2002.

[21] T Lunt, O Waldmann, and G Fussmann. Laser induced fluorescence measurements in an argon plasma in front of a tungsten target under oblique incidence. In 35th EPS Conference on Plasma Physics and 10th International Workshop on Fast Ignition of Fusion Targets. European Physical Society, 2008 . 
[22] M Umair Siddiqui, Cory D Jackson, Justin F Kim, and Noah Hershkowitz. Direct measurements of ion dynamics in collisional magnetic presheaths. Physics of Plasmas, 21(10):102103, 2014.

[23] M Umair Siddiqui, Derek S Thompson, Cory D Jackson, Justin F Kim, Noah Hershkowitz, and Earl E Scime. Models, assumptions, and experimental tests of flows near boundaries in magnetized plasmas. Physics of Plasmas, 23 (5):057101, 2016. URL https://aip.scitation.org/doi/abs/10.1063/ 1.4943523 .

[24] Nicolas Claire, Gerard Bachet, Ulrich Stroth, and F Doveil. Laser-inducedfluorescence observation of ion velocity distribution functions in a plasma sheath. Physics of plasmas, 13(6):062103, 2006.

[25] Derek S Thompson, Thomas E Steinberger, Amy M Keesee, and Earl E Scime. Laser induced fluorescence of Ar-I metastables in the presence of a magnetic field. Plasma Sources Science and Technology, 27:065007, 2018. URL http://iopscience.iop.org/article/10.1088/1361-6595/ aac963/meta.

[26] TW Chevalier, Umran S Inan, and Timothy F Bell. Fluid simulation of the collisionless plasma sheath surrounding an electric dipole antenna in the inner magnetosphere. Radio Science, 45(1), 2010.

[27] VI Demidov, Svetlana V Ratynskaia, and K Rypdal. Electric probes for plasmas: The link between theory and instrument. Review of scientific instruments, 73(10):3409-3439, 2002.

[28] Mike Kotschenreuther, Prashant Valanju, Brent Covele, and Swadesh Mahajan. Magnetic geometry and physics of advanced divertors: The $\mathrm{x}-$ divertor and the snowflake. Physics of Plasmas, 20(10):102507, 2013. doi: 10.1063/1.4824735. URL https://doi.org/10.1063/1.4824735.

[29] PC Stangeby and GM McCracken. Plasma boundary phenomena in tokamaks. Nuclear Fusion, 30(7):1225, 1990.

[30] PC Stangeby. The chodura sheath for angles of a few degrees between the magnetic field and the surface of divertor targets and limiters. Nuclear Fusion, 52(8):083012, 2012.

[31] Manuel Gamero-Castaño and I Katz. Estimation of Hall thruster erosion using HPHall. Cleveland, OH, 2005. 29th International Electric Propulsion Conference Paper No. IEPC 2005-303.

[32] Ioannis G. Mikellides, Ira Katz, Richard R. Hofer, and Dan M. Goebel. Magnetic shielding of a laboratory Hall thruster. I. Theory and validation. Journal of Applied Physics, 115(4):043303, 2014. doi: 10.1063/1.4862313. URL https://doi .org/10.1063/1.4862313. 
[33] Richard R. Hofer, Dan M. Goebel, Ioannis G. Mikellides, and Ira Katz. Magnetic shielding of a laboratory Hall thruster. II. Experiments. Journal of Applied Physics, 115(4):043304, 2014. doi: 10.1063/1.4862314. URL https: //doi.org/10.1063/1.4862314.

[34] Gary D. Porter, S. L. Allen, M. Brown, M. E. Fenstermacher, D. N. Hill, R. A. Jong, A. W. Leonard, D. Nilson, M. E. Rensink, T. D. Rognlien, G. R. Smith, and the DIII-D Team. Simulation of experimentally achieved DIII-D detached plasmas using the UEDGE code. Physics of Plasmas, 3(5): 1967-1975, 1996. doi: 10.1063/1.871993. URL https://doi.org/10.1063/ 1.871993.

[35] Shannon Cheng and Manuel Martinez-Sanchez. Hybrid particle-in-cell erosion modeling of two Hall thrusters. Journal of Propulsion and Power, 24 (5):987-998, 2008.

[36] M. Ono, S.M. Kaye, Y.-K.M. Peng, G. Barnes, W. Blanchard, M.D. Carter, J. Chrzanowski, L. Dudek, R. Ewig, D. Gates, R.E. Hatcher, T. Jarboe, S.C. Jardin, D. Johnson, R. Kaita, M. Kalish, C.E. Kessel, H.W. Kugel, R. Maingi, R. Majeski, J. Manickam, B. McCormack, J. Menard, D. Mueller, B.A. Nelson, B.E. Nelson, C. Neumeyer, G. Oliaro, F. Paoletti, R. Parsells, E. Perry, N. Pomphrey, S. Ramakrishnan, R. Raman, G. Rewoldt, J. Robinson, A.L. Roquemore, P. Ryan, S. Sabbagh, D. Swain, E.J. Synakowski, M. Viola, M. Williams, J.R. Wilson, and NSTX Team. Exploration of spherical torus physics in the NSTX device. Nuclear Fusion, 40(3Y):557, 2000. URL http://stacks.iop.org/0029-5515/40/i=3Y/a=316.

[37] J.-W. Ahn and G.F. Counsell. Experimental investigations of the SOL plasma in the MAST tokamak. Journal of Nuclear Materials, 290-293:820 824, 2001. ISSN 0022-3115. doi: https://doi.org/10.1016/S0022-3115(00) 00466-9. URL http://www.sciencedirect.com/science/article/pii/ S0022311500004669. 14th Int. Conf. on Plasma-Surface Interactions in Controlled Fusion Devices.

[38] K.E. Thome, M.W. Bongard, J.L. Barr, G.M. Bodner, M.G. Burke, R.J. Fonck, D.M. Kriete, J.M. Perry, J.A. Reusch, and D.J. Schlossberg. H-mode plasmas at very low aspect ratio on the pegasus toroidal experiment. Nuclear Fusion, 57(2):022018, 2017. URL http://stacks.iop.org/0029-5515/57/ $i=2 / a=022018$.

[39] Franklin R. Chang-Díaz. Plasma propulsion for interplanetary flight. Thin Solid Films, 506-507:449 - 453, 2006. ISSN 0040-6090. doi: https:// doi.org/10.1016/j.tsf.2005.08.324. URL http://www.sciencedirect.com/ science/article/pii/S0040609005012496. The Joint Meeting of 7th APCPST (Asia Pacific Conference on Plasma Science and Technology) and 17th SPSM (Symposium on Plasma Science for Materials).

[40] J. Rapp, T. M. Biewer, T. S. Bigelow, J. B. O. Caughman, R. C. Duckworth, R. J. Ellis, D. R. Giuliano, R. H. Goulding, D. L. Hillis, R. H. Howard, T. L. 
Lessard, J. D. Lore, A. Lumsdaine, E. J. Martin, W. D. McGinnis, S. J. Meitner, L. W. Owen, H. B. Ray, G. C. Shaw, and V. K. Varma. The development of the material plasma exposure experiment. IEEE Transactions on Plasma Science, 44(12):3456-3464, Dec 2016. ISSN 0093-3813. doi: 10.1109/TPS.2016.2628326.

[41] Ivo Furno, Riccardo Agnello, Basil Duval, Claudio Marini, Alan Howling, Rémy Jacquier, Philippe Guittienne, Fantz Usel, Wünderlich Dirk, Simonin Alain, and Béchu Stephane. A novel helicon plasma source for negative ion beams for fusion. 2016.

[42] G N Harding and P C Thonemann. A study of helicon waves in indium. Proceedings of the Physical Society, 85(2):317, 1965. URL http://stacks . iop.org/0370-1328/85/i=2/a=313.

[43] Charles Rudolf Legéndy. Macroscopic theory of helicons. Physical Review, 135(6A):A1713, 1964.

[44] D Formato and A Gilardini. Propagation characteristics of magneto-ionic plasma columns. J. Research Natl. Bur. Standards, 66, 1962.

[45] J. P. Klozenberg, B. McNamara, and P. C. Thonemann. The dispersion and attenuation of helicon waves in a uniform cylindrical plasma. Journal of Fluid Mechanics, 21(3):545-563, 1965. doi: 10.1017/S0022112065000320.

[46] Rod W Boswell and Francis F Chen. Helicons - the early years. IEEE Transactions on Plasma Science, 25(6):1229-1244, 1997.

[47] LRO Storey. An investigation of whistling atmospherics. Phil. Trans. R. Soc. Lond. A, 246(908):113-141, 1953.

[48] RM Gallet, JM Richardson, B Wieder, GD Ward, and GN Harding. Microwave whistler mode propagation in a dense laboratory plasma. Physical Review Letters, 4(7):347, 1960.

[49] Paul A Keiter, Earl E Scime, and Matthew M Balkey. Frequency dependent effects in helicon plasmas. Physics of Plasmas, 4(7):2741-2747, 1997.

[50] RW Boswell. Plasma production using a standing helicon wave. Physics Letters A, 33(7):457-458, 1970.

[51] L Porte, S M Yun, D Arnush, and F F Chen. Superiority of half-wavelength helicon antennae. Plasma Sources Science and Technology, 12(2):287, 2003. URL http://stacks.iop.org/0963-0252/12/i=2/a=320.

[52] J A Lehane and P C Thonemann. An experimental study of helicon wave propagation in a gaseous plasma. Proceedings of the Physical Society, 85(2): 301, 1965. URL http://stacks. iop.org/0370-1328/85/i=2/a=312. 
[53] M Manentea, M Walkerb, J Carlssonc, C Bramantid, S Rocca, D Currelia, Y Guçlua, and D Pavarina. Feasibility study of low power helicon thruster. In 5th International spacecraft Propulsion Conference, Crete, Greece, May, 2008 .

[54] Derek S. Thompson, Riccardo Agnello, Ivo Furno, Alan Howling, Rémy Jacquier, Gennady Plyushchev, and Earl E. Scime. Ion heating and flows in a high power helicon source. Physics of Plasmas, 24(6):063517, 2017. doi: 10.1063/1.4985328. URL https://doi.org/10.1063/1.4985328.

[55] J. Rapp, T. M. Biewer, T. Bigelow, J. B. O. Caughman, R. Duckworth, D. Giuliano, R. H. Goulding, D. L. Hillis, R. Howard, R. J. Ellis, T. Lessard, J. D. Lore, A. Lumsdaine, E. Martin, W. D. McGinnis, S. J. Meitner, L. W. Owen, H. Ray, G. Shaw, and V. Varma. The Material Plasma Exposure eXperiment MPEX: Pre-design, development and testing of source concept. In 2015 IEEE 26th Symposium on Fusion Engineering (SOFE), pages 1-8, May 2015. doi: 10.1109/SOFE.2015.7482351.

[56] GR Tynan, AD Bailey III, GA Campbell, R Charatan, A De Chambrier, G Gibson, DJ Hemker, K Jones, A Kuthi, C Lee, et al. Characterization of an azimuthally symmetric helicon wave high density plasma source. Journal of Vacuum Science \& Technology A: Vacuum, Surfaces, and Films, 15(6): 2885-2892, 1997.

[57] C Charles. Plasmas for spacecraft propulsion. Journal of Physics D: Applied Physics, 42(16):163001, 2009. URL http://stacks.iop.org/0022-3727/ $42 / i=16 / a=163001$.

[58] Kazunori Takahashi, Trevor Lafleur, Christine Charles, Peter Alexander, and Rod W. Boswell. Electron diamagnetic effect on axial force in an expanding plasma: experiments and theory. Phys. Rev. Lett., 107:235001, Nov 2011. doi: 10.1103/PhysRevLett.107.235001. URL https://link.aps.org/doi/ 10.1103/PhysRevLett.107.235001.

[59] Earl E Scime, Paul A Keiter, Matt M Balkey, John L Kline, X Sun, Amy M Keesee, Robert A Hardin, I A Biloiu, S Houshmandyar, Saikat Chakraborty Thakur, Jerry Carr Jr., Matt Galante, Dustin McCarren, and Stephanie Sears. The Hot hELIcon eXperiment (HELIX) and the Large Experiment on Instabilities and Anisotropy (LEIA). Journal of Plasma Physics, 81(1), 2015 .

[60] Francis F Chen and Rod W Boswell. Helicons - the past decade. IEEE Transactions on Plasma Science, 25(6):1245-1257, 1997.

[61] EE Scime, AM Keesee, and RW Boswell. Mini-conference on helicon plasma sources. Physics of Plasmas, 15(5):058301, 2008.

[62] Francis F Chen. Helicon discharges and sources: a review. Plasma Sources Science and Technology, 24(1):014001, 2015. URL http://stacks.iop. org $/ 0963-0252 / 24 / i=1 / a=014001$. 
[63] Francis F Chen. Plasma ionization by helicon waves. Plasma Physics and Controlled Fusion, 33(4):339, 1991.

[64] T Shoji, Y Sakawa, S Nakazawa, K Kadota, and T Sato. Plasma production by helicon waves. Plasma Sources Science and Technology, 2(1):5, 1993. URL http://stacks.iop.org/0963-0252/2/i=1/a=002.

[65] D Gary Swanson. Plasma Waves. IOP Publishing (New York), 1989.

[66] Francis F Chen. Introduction to Plasma Physics and Controlled Fusion Volume 1: plasma Physics. Springer, 2 edition, 2006.

[67] W Baumjohann and RA Treumann. Basic space plasma physics. Imperial College Press, London, 1997.

[68] R W Boswell. Very efficient plasma generation by whistler waves near the lower hybrid frequency. Plasma Physics and Controlled Fusion, 26(10):1147, 1984. URL http://stacks.iop.org/0741-3335/26/i=10/a=001.

[69] Paul A Keiter, Earl E Scime, and Matthew M Balkey. Frequency dependent effects in helicon plasmas. Physics of Plasmas, 4(7):2741-2747, 1997.

[70] Francis F. Chen and David D. Blackwell. Upper limit to Landau damping in helicon discharges. Phys. Rev. Lett., 82:2677-2680, Mar 1999. doi: 10.1103/PhysRevLett.82.2677. URL https://link.aps.org/doi/10. 1103/PhysRevLett.82.2677.

[71] Evan M Aguirre, Earl E Scime, Derek S Thompson, and Timothy N Good. Spatial structure of ion beams in an expanding plasma. Physics of Plasmas, 24(12):123510, 2017. URL https://aip.scitation.org/doi/abs/ $10.1063 / 1.5003722$.

[72] JL Kline, EE Scime, RF Boivin, AM Keesee, X Sun, and VS Mikhailenko. RF absorption and ion heating in helicon sources. Physical review letters, 88(19): 195002, 2002. URL https://doi.org/10.1103/PhysRevLett.88.195002.

[73] Saikat Chakraborty Thakur, Christian Brandt, Lang Cui, Jordan James Gosselin, and George R Tynan. Formation of the blue core in argon helicon plasma. IEEE Transactions on Plasma Science, 43(8):2754-2759, 2015. URL https://doi.org/10.1109/TPS.2015.2446537.

[74] Saikat Chakraborty Thakur, Min Xu, Peter Manz, Nicolas Fedorczak, Chris Holland, and George R Tynan. Suppression of drift wave turbulence and zonal flow formation by changing axial boundary conditions in a cylindrical magnetized plasma device. Physics of Plasmas, 20(1):012304, 2013.

[75] P. Vaezi, C. Holland, S. C. Thakur, and G. R. Tynan. Understanding the impact of insulating and conducting endplate boundary conditions on turbulence in CSDX through nonlocal simulations. Physics of Plasmas, 24(4): 042306, 2017. doi: 10.1063/1.4980843. URL https://doi.org/10.1063/1. 4980843. 
[76] Earl Scime, Robert Hardin, C Biloiu, AM Keesee, and Xuan Sun. Flow, flow shear, and related profiles in helicon plasmas. Physics of plasmas, 14 (4):043505, 2007. URL https://aip.scitation.org/doi/abs/10.1063/ 1.2716687 .

[77] S. Chakraborty Thakur, D. McCarren, T. Lee, N. Fedorczak, P. Manz, E. E. Scime, G. R. Tynan, and M. Xu. Laser induced fluorescence measurements of ion velocity and temperature of drift turbulence driven sheared plasma flow in a linear helicon plasma device. Physics of Plasmas, 19(8):082102, 2012. doi: 10.1063/1.4742178. URL https://doi.org/10.1063/1.4742178.

[78] S Chakraborty Thakur, K Adriany, JJ Gosselin, J McKee, EE Scime, SH Sears, and GR Tynan. Laser induced fluorescence measurements of axial velocity, velocity shear, and parallel ion temperature profiles during the route to plasma turbulence in a linear magnetized plasma device. Review of Scientific Instruments, 87(11):11E513, 2016. URL https: //aip.scitation.org/doi/abs/10.1063/1.4959275.

[79] J Gilland, R Breun, and N Hershkowitz. Neutral pumping in a helicon discharge. Plasma Sources Science and Technology, 7(3):416, 1998. URL http://stacks.iop.org/0963-0252/7/i=3/a=020.

[80] A M Keesee and E E Scime. Neutral density profiles in argon helicon plasmas. Plasma Sources Science and Technology, 16(4):742, 2007. URL http:// stacks. iop.org/0963-0252/16/i=4/a=008.

[81] A Aanesland, L Liard, G Leray, J Jolly, and P Chabert. Direct measurements of neutral density depletion by two-photon absorption laser-induced fluorescence spectroscopy. Applied Physics Letters, 91(12):121502, 2007.

[82] Masashi Shimada, George R Tynan, and Robert Cattolica. Neutral gas density depletion due to neutral gas heating and pressure balance in an inductively coupled plasma. Plasma Sources Science and Technology, 16(1): 193, 2006.

[83] L Liard, Ane Aanesland, and Pascal Chabert. Dynamics of neutral gas depletion investigated by time-and space-resolved measurements of xenon atom ground state density. Journal of Physics D: Applied Physics, 45(23): 235201, 2012.

[84] RM Magee, ME Galante, N Gulbrandsen, DW McCarren, and EE Scime. Direct measurements of the ionization profile in krypton helicon plasmas. Physics of Plasmas, 19(12):123506, 2012. URL https://aip.scitation. org/doi/abs/10.1063/1.4772060.

[85] Francis F Chen. Capacitor tuning circuits for inductive loads. UCLA Report, 1992.

[86] John Taylor. Introduction to error analysis, the study of uncertainties in physical measurements. University Science Books, 1997. 
[87] User's Manual Model 455 DSP Gaussmeter. Lake Shore Cryotronics, 575 McCorkle Blvd, 1.5 edition, May 2014.

[88] I Langmuir, HM Mott-Smith, I Langmuir, HM Mott-Smith, I Langmuir, HM Mott-Smith, I Langmuir, and HM Mott-Smith Gen. Electric Rev., 27 (1924). Gen. Electric Rev, 27:449-810, 1924.

[89] Harold M Mott-Smith and Irving Langmuir. The theory of collectors in gaseous discharges. Physical review, 28(4):727, 1926.

[90] Ira M Cohen. Saturation currents to Langmuir probes in a collisiondominated plasma with uniform magnetic field. The Physics of Fluids, 12 (11):2356-2361, 1969.

[91] Juan R Sanmartín. Theory of a probe in a strong magnetic field. The Physics of Fluids, 13(1):103-116, 1970.

[92] Ian G Brown, Alan B Compher, and Wulf B Kunkel. Response of a Langmuir probe in a strong magnetic field. Physics of Fluids, 14(7):1377-1383, 1971.

[93] PM Chung, L Talbot, and KJ Touryan. Electrostatic Probes in Stationary and Flowing Plasmas: theory and Application. Springer-Verlag, 1975.

[94] Tsv K Popov, M Dimitrova, P Ivanova, J Kovacic, T Gyergyek, R Dejarnac, J Stockel, MA Pedrosa, D Lopez-Bruna, and C Hidalgo. Advances in Langmuir probe diagnostics of the plasma potential and electron-energy distribution function in magnetized plasma. Plasma Sources Science and Technology, $25(3), 2016$.

[95] Isaac D Sudit and Francis F Chen. RF compensated probes for high-density discharges. Plasma Sources Science and Technology, 3(2):162, 1994.

[96] VA Godyak and VI Demidov. Probe measurements of electron-energy distributions in plasmas: what can we measure and how can we achieve reliable results? Journal of Physics D: Applied Physics, 44(23):233001, 2011.

[97] MA Jaworski, MG Bell, TK Gray, R Kaita, J Kallman, HW Kugel, B LeBlanc, AG McLean, SA Sabbagh, VA Soukhanovskii, et al. Modification of the electron energy distribution function during lithium experiments on the National Spherical Torus Experiment. Fusion Engineering and Design, 87(10):1711-1718, 2012.

[98] Noah Hershkowitz. How Langmuir probes work. In Orlando Auciello and Daniel L Flamm, editors, Plasma Diagnostics, volume 1, pages 113-183. Academic Press, 1989.

[99] Ian H Hutchinson. Principles of Plasma Diagnostics, volume 44. IOP Publishing, 2002.

[100] Francis F Chen. Numerical computations for ion probe characteristics in a collisionless plasma. Journal of Nuclear Energy. Part C, Plasma Physics, Accelerators, Thermonuclear Research, 7(1):47, 1965. 
[101] Francis F Chen. Langmuir probe diagnostics. In IEEE-ICOPS Meeting, Jeju, Korea, volume 2, 2003.

[102] Carpenter Technology Corporation. Carpenter's stainless steel blue book. Technical report, Carpenter Technology Corporation, 2012.

[103] Akio Koyama, Takashi Shikata, and Hideo Sakairi. Secondary electron emission from al, cu, ag and au metal targets under proton bombardment. Japanese Journal of Applied Physics, 20(1):65, 1981.

[104] E Oyarzabal, AB Martin-Rojo, and FL Tabarés. Electron-induced secondary electron emission coefficient of lithium, tungsten and stainless steel surfaces exposed to low-pressure plasmas. Journal of Nuclear Materials, 452(1):3740, 2014.

[105] V Baglin, J Bojko, C Scheuerlein, Oswald Gröbner, M Taborelli, Bernard Henrist, and Noël Hilleret. The secondary electron yield of technical materials and its variation with surface treatments. In Proceedings of EPAC 2000, 2000 .

[106] VA Godyak, RB Piejak, and BM Alexandrovich. Measurement of electron energy distribution in low-pressure RF discharges. Plasma sources science and technology, 1(1):36, 1992.

[107] RB Piejak, VA Godyak, and BM Alexandrovich. A simple analysis of an inductive RF discharge. Plasma sources science and technology, 1(3):179, 1992.

[108] VA Godyak, RB Piejak, and BM Alexandrovich. Electron energy distribution function measurements and plasma parameters in inductively coupled argon plasma. Plasma Sources Science and Technology, 11(4):525, 2002.

[109] Paul A Keiter. Experimental Investigation of Ion Temperature Anisotropy Driven Instabilities in a High Beta Plasma. PhD thesis, West Virginia University, 1999.

[110] Amy M Keesee. Neutral Density Profiles in Argon Helicon Plasmas. PhD thesis, West Virginia University, 2006.

[111] Lenox-Fugle International, Inc. 1071 N. Grand Ave. 115, Nogales, AZ 85621. (520) 287-5847.

[112] Cyrus Nourgostar. (personal correspondence). (Personal correspondence), 2013.

[113] VA Godyak and BM Alexandrovich. Comparative analyses of plasma probe diagnostics techniques. Journal of Applied Physics, 118(23):233302, 2015.

[114] JE Allen, RLF Boyd, and P Reynolds. The collection of positive ions by a probe immersed in a plasma. Proceedings of the Physical Society. Section B, $70(3): 297,1957$. 
[115] Francis F Chen, John D Evans, and Wade Zawalski. Calibration of Langmuir probes against microwaves and plasma oscillation probes. Plasma Sources Science and Technology, 21(5):055002, 2012.

[116] Ira B Bernstein and Irving N Rabinowitz. Theory of electrostatic probes in a low-density plasma. The Physics of Fluids, 2(2):112-121, 1959.

[117] James G Laframboise. Theory of spherical and cylindrical Langmuir probes in a collisionless, Maxwellian plasma at rest. Technical report, TORONTO UNIV DOWNSVIEW (ONTARIO) INST FOR AEROSPACE STUDIES, 1966.

[118] Juan Rn Sanmartín and Robert D Estes. The orbital-motion-limited regime of cylindrical Langmuir probes. Physics of Plasmas, 6(1):395-405, 1999.

[119] Robert D Estes and Juan Rn Sanmartín. Cylindrical Langmuir probes beyond the orbital-motion-limited regime. Physics of Plasmas, 7(10):43204325, 2000.

[120] Francis F Chen, John D Evans, and Donald Arnush. A floating potential method for measuring ion density. Physics of Plasmas, 9(4):1449-1455, 2002.

[121] Michael A Lieberman and Alan J Lichtenberg. Principles of plasma discharges and materials processing. John Wiley \& Sons, 2005.

[122] VA Godyak and BM Alexandrovich. Langmuir paradox revisited. Plasma Sources Science and Technology, 24(5):052001, 2015.

[123] J D Huba. NRL (Naval Research Laboratory) plasma formulary, revised. Naval Research Lab. Report, 2016.

[124] Av V Phelps. The application of scattering cross sections to ion flux models in discharge sheaths. Journal of Applied Physics, 76(2):747-753, 1994.

[125] Billy J Nichols and Fred C Witteborn. Measurements of resonant charge exchange cross sections in nitrogen and argon between 0.5 and $17 \mathrm{ev}$. Technical report, NASA, 1966.

[126] Mari Johan Druyvesteyn. Der niedervoltbogen. Zeitschrift für Physik A Hadrons and Nuclei, 64(11):781-798, 1930.

[127] VI Demidov, SV Ratynskaia, RJ Armstrong, and K Rypdal. Probe measurements of electron energy distributions in a strongly magnetized low-pressure helium plasma. Physics of Plasmas, 6(1):350-358, 1999.

[128] Tsv K Popov, P Ivanova, J Stöckel, and R Dejarnac. Electron energy distribution function, plasma potential and electron density measured by Langmuir probe in tokamak edge plasma. Plasma Physics and Controlled Fusion, 51(6):065014, 2009.

[129] VA Godyak, RB Piejak, and BM Alexandrovich. Probe diagnostics of nonMaxwellian plasmas. Journal of applied physics, 73(8):3657-3663, 1993. 
[130] Frið rik Magnus and Jon Tomas Gudmundsson. Digital smoothing of the Langmuir probe I-V characteristic. Review of Scientific Instruments, 79(7): 073503, 2008.

[131] D Yordanov, St Lishev, and A Shivarova. How does a probe inserted into the discharge influence the plasma structure? Journal of Applied Physics, 119(18):183302, 2016.

[132] AR Hoskinson and Noah Hershkowitz. Effect of finite length on the currentvoltage characteristic of a cylindrical Langmuir probe in a multidipole plasma chamber. Plasma Sources Science and Technology, 15(1):85, 2006.

[133] Julius S Bendat and Allan G Piersol. Random data: analysis and measurement procedures, volume 729. John Wiley \& Sons, 4th edition, 2011.

[134] MathWorks. Periodogram power spectral density estimate - matlab periodogram, 1997. URL https://www.mathworks.com/help/signal/ref/ periodogram.html.

[135] R. A. Stern and J. A. Johnson. Plasma ion diagnostics using resonant fluorescence. Phys. Rev. Lett., 34(25):1548-1551, Jun 1975.

[136] J. Bokor, R. R. Freeman, J. C. White, and R. H. Storz. Two-photon excitation of the $n=3$ level in $\mathrm{h}$ and d atoms. Phys. Rev. A, 24(1):612-614, Jul 1981.

[137] Toshinori Kajiwara, Tatsuya Shinkawa, Kiichiro Uchino, Mitsuharu Masuda, Katsunori Muraoka, Tatsuo Okada, Mitsuo Maeda, Sigeru Sudo, and Tokuhiro Obiki. Application of two-photon-excited laser-induced fluorescence to atomic hydrogen measurements in the edge region of hightemperature plasmas. Review of Scientific Instruments, 62(10):2345-2349, 1991. doi: 10.1063/1.1142296. URL http://dx.doi.org/10.1063/1. 1142296.

[138] A. D. Bailey, R. A. Stern, and P. M. Bellan. Measurement of coherent driftwave ion-fluid velocity field when ion dynamics are stochastic. Phys. Rev. Lett., 71:3123-3126, Nov 1993. doi: 10.1103/PhysRevLett.71.3123. URL https://link.aps.org/doi/10.1103/PhysRevLett.71.3123.

[139] Fred M. Levinton and Fedor Trintchouk. Visualization of plasma turbulence with laser-induced fluorescence (invited). Review of Scientific Instruments, 72(1):898-905, 2001. doi: 10.1063/1.1321005. URL http://dx.doi.org/ $10.1063 / 1.1321005$.

[140] A. K. Hansen, Matthew Galante, Dustin McCarren, Stephanie Sears, and E. E. Scime. Simultaneous two-dimensional laser-induced-fluorescence measurements of argon ions. Review of Scientific Instruments, 81(10):10D701, 2010. doi: 10.1063/1.3460630. URL http://dx.doi.org/10.1063/1. 3460630 . 
[141] M. Zintl and R. McWilliams. Improved optical tomography device. Review of Scientific Instruments, 65(8):2574-2579, 1994. doi: 10.1063/1.1144653. URL http://dx.doi.org/10.1063/1.1144653.

[142] G. D. Severn, D. A. Edrich, and R. McWilliams. Argon ion laser-induced fluorescence with diode lasers. Review of Scientific Instruments, 69(1):1015, 1998. doi: 10.1063/1.1148472. URL http://dx.doi.org/10.1063/1. 1148472 .

[143] Amy M. Keesee, Earl E. Scime, and Robert F. Boivin. Laser-induced fluorescence measurements of three plasma species with a tunable diode laser. Review of Scientific Instruments, 75(10):4091-4093, 2004. doi: 10.1063/1.1787166. URL http://dx.doi.org/10.1063/1.1787166.

[144] RF Boivin. Study of the different line broadening mechanisms for the laser induced fluorescence diagnostic of the helix and leia plasmas, pl-039. Technical report, West Virginia University Plasma Physics Lab, 2002.

[145] RF Boivin. West virginia university plasma physics lab report pl-050. Technical report, West Virginia University Plasma Physics Lab, 2003.

[146] Derek S. Thompson, Miguel F. Henriquez, Earl E. Scime, and Timothy N. Good. Confocal laser induced fluorescence with comparable spatial localization to the conventional method. Review of Scientific Instruments, 88(10): 103506, 2017. doi: 10.1063/1.4991637. URL https://doi.org/10.1063/1. 4991637.

[147] Grant R Fowles. Introduction to Modern Optics. Courier Corporation, 1975.

[148] 2014. URL https://www.thorlabs.com/newgrouppage9.cfm? objectgroup_id=5510.

[149] 2014. URL https://www.thorlabs.com/newgrouppage9.cfm? objectgroup_id=8635\&pn=WPQ10ME-670\#8637.

[150] 2014. URL https://www.thorlabs.com/newgrouppage9.cfm? objectgroup_id=1400.

[151] R VanDervort, D Elliott, D McCarren, J McKee, M Soderholm, S Sears, and E Scime. Optimization of confocal laser induced fluorescence in a plasma. Review of Scientific Instruments, 85(11):11E408, 2014.

[152] C. M. Ferreira, J. Loureiro, and A. Ricard. Populations in the metastable and the resonance levels of argon and stepwise ionization effects in a lowpressure argon positive column. Journal of Applied Physics, 57(1):82-90, 1985. doi: 10.1063/1.335400. URL https://doi.org/10.1063/1.335400.

[153] Brian K McMillin and Michael R Zachariah. Two-dimensional argon metastable density measurements in a radio frequency plasma reactor by planar laser-induced fluorescence imaging. Journal of applied physics, 77 (11):5538-5544, 1995. 
[154] G. A. Hebner. Spatially resolved, excited state densities and neutral and ion temperatures in inductively coupled argon plasmas. Journal of Applied Physics, 80(5):2624-2636, 1996. doi: 10.1063/1.363178. URL http://dx. doi.org/10.1063/1.363178.

[155] Brian K. McMillin and M. R. Zachariah. Two-dimensional laser-induced fluorescence imaging of metastable density in low-pressure radio frequency argon plasmas with added o2, cl2, and cf4. Journal of Applied Physics, 79(1): 77-85, 1996. doi: 10.1063/1.360793. URL http://dx.doi.org/10.1063/1. 360793.

[156] Zachary D. Short, M. Umair Siddiqui, Miguel F. Henriquez, John S. McKee, and Earl E. Scime. A novel laser-induced fluorescence scheme for ar-i in a plasma. Review of Scientific Instruments, 87(1):013505, 2016. doi: 10.1063/ 1.4939909. URL http://dx.doi.org/10.1063/1.4939909.

[157] McPherson Model 209 Specification Sheet.

[158] R. F. Boivin and E. E. Scime. Laser induced fluorescence in Ar and He plasmas with a tunable diode laser. Review of Scientific Instruments, 74 (10):4352-4360, 2003. doi: 10.1063/1.1606095. URL http://dx.doi.org/ $10.1063 / 1.1606095$.

[159] M. J. Goeckner, J. Goree, and T. E. Sheridan. Saturation broadening of laserinduced fluorescence from plasma ions. Review of Scientific Instruments, 64 (4):996-1000, 1993. doi: 10.1063/1.1144103. URL https://doi.org/10. $1063 / 1.1144103$.

[160] David N Hill, Steven Fornaca, and Michael G Wickham. Single frequency scanning laser as a plasma diagnostic. Review of Scientific Instruments, 54 (3):309-314, 1983.

[161] Yuri Ralchenko. Nist atomic spectra database. Memorie della Societa Astronomica Italiana Supplementi, 8:96, 2005.

[162] Vahid Vahedi and Maheswaran Surendra. A monte carlo collision model for the particle-in-cell method: applications to argon and oxygen discharges. Computer Physics Communications, 87(1-2):179-198, 1995.

[163] M. Surendra, D. B. Graves, and G. M. Jellum. Self-consistent model of a direct-current glow discharge: treatment of fast electrons. Phys. Rev. A, 41: 1112-1125, Jan 1990. doi: 10.1103/PhysRevA.41.1112. URL https : //link. aps.org/doi/10.1103/PhysRevA.41.1112.

[164] Rinat Khaziev. MULTISCALE NUMERICAL SIMULATIONS OF THE MAGNETIZED PLASMA SHEATH WITH MASSIVELY PARALLEL ELECTROSTATIC PARTICLE-IN-CELL CODE. PhD thesis, University of Illinois at Urbana-Champgain, 2018.

[165] Scott D Baalrud, JD Callen, and CC Hegna. Kinetic theory of instabilityenhanced collisional effects. Physics of Plasmas, 17(5):055704, 2010. 
[166] Jeffrey P Freidberg. Plasma physics and fusion energy. Cambridge university press, 2008.

[167] Chi-Shung Yip, Noah Hershkowitz, and Greg Severn. Verifying effects of instability enhanced ion-ion coulomb collisions on ion velocity distribution functions near the sheath edge in low temperature plasmas. Plasma Sources Science and Technology, 24(1):015018, 2014.

[168] K-U Riemann. Theory of the collisional presheath in an oblique magnetic field. Physics of plasmas, 1(3):552-558, 1994.

[169] T. M. G. Zimmermann, M. Coppins, and J. E. Allen. Fluid model of the boundary of a one-dimensional plasma under the influence of an oblique magnetic field for a wide range of collisionality. Physics of Plasmas, 15(7): 072301, 2008. doi: 10.1063/1.2946436. URL https://doi .org/10.1063/1. 2946436.

[170] M Umair Siddiqui. Presheath and Double Layer Structures in an Argon Helicon Plasma Source. PhD thesis, The University of Wisconsin-Madison, 2014 .

[171] Jin-Young Bang and Chin-Wook Chung. A numerical method for determining highly precise electron energy distribution functions from Langmuir probe characteristics. Physics of Plasmas, 17(12):123506, 2010.

[172] Jin Young Bang, Aram Kim, and Chin Wook Chung. Improved measurement method for electron energy distribution functions with high accuracy and reliability. Physics of Plasmas, 17(6):064502, 2010.

[173] MathWorks. Sliding window method and exponential weighting method, 1997. URL https://www.mathworks.com/help/dsp/ug/ sliding-window-method-and-exponential-weighting-method.html.

[174] MathWorks. Blackman window - matlab blackman, 1997. URL https: //www . mathworks. com/help/signal/ref/blackman.html. 